\title{
Development of High-Intensity D-D and D-T Neutron Sources and Neutron Filters for Medical and Industrial Applications.
}

\author{
by
}

\author{
Jérôme Maurice Verbeke
}

B.S. (Université Catholique de Louvain, Louvain-la-Neuve, Belgium) 1995

M.S. (University of California, Berkeley) 1997

A dissertation submitted in partial satisfaction of the

requirements for the degree of

Doctor of Philosophy

in

Engineering - Nuclear Engineering

in the

GRADUATE DIVISION

of the

UNIVERSITY OF CALIFORNIA, BERKELEY

\author{
Committee in charge: \\ Professor Jasmina Vujic, Chair \\ Professor Paul Chambré \\ Professor Wulf Kunkel \\ Doctor Ka-Ngo Leung
}

Spring 2000 
The dissertation of Jérôme Maurice Verbeke is approved:

\begin{tabular}{cc}
\hline Chair & Date \\
\hline & Date \\
\hline & Date \\
\hline & \\
\hline Date
\end{tabular}

University of California, Berkeley

Spring 2000 
Development of High-Intensity D-D and D-T Neutron Sources and Neutron Filters for Medical and Industrial Applications.

Copyright 2000

by

Jérôme Maurice Verbeke 


\begin{abstract}
Development of High-Intensity D-D and D-T Neutron Sources and Neutron Filters for Medical and Industrial Applications.

by

Jérôme Maurice Verbeke

Doctor of Philosophy in Engineering - Nuclear Engineering

University of California, Berkeley

Professor Jasmina Vujic, Chair
\end{abstract}

This thesis consists of three main parts. The first part is related to boron neutron capture therapy (BNCT), the second part to boron neutron capture synovectomy (BNCS), and the third part to the neutron generator development.

The first part is composed of two chapters. A monoenergetic neutron beam simulation study is carried out in the first chapter to determine the most suitable neutron energy for treatment of shallow and deep-seated brain tumors in the context of BNCT. Two figures-of-merit - the absorbed skin dose and the absorbed tumor dose at a given depth in the brain - are used to measure the neutron beam quality. Based on the results of this study, moderators, reflectors and delimiters are designed and optimized in the second chapter to moderate the high-energy neutrons from the fusion reactions D-D and D-T producing $2.45 \mathrm{MeV}$ and $14.1 \mathrm{MeV}$ neutrons, respectively - down to a suitable energy spectrum. Two different computational models (MCNP and BNCT_RTPE) have been used to study the dose distribution in the brain. With the optimal beam-shaping assembly, a 1.5-A mixed deuteron/triton beam of energy $150 \mathrm{keV}$ accelerated onto a titanium target leads to a treatment time of $\sim 45 \mathrm{~min}$. The dose near the center of the brain obtained with this configuration is more than $65 \%$ higher than the dose from a typical spectrum produced by the Brookhaven Medical Research Reactor, and is comparable to the dose obtained by other accelerator-produced neutron beams.

The second part is composed of two chapters. In the first one, a monoenergetic 
neutron beam simulation study is carried out to determine the optimal neutron energy for treatment of rheumatoid arthritis. Two figures-of-merit are used to measure the neutron beam quality, the ratio of the synovium absorbed dose to the skin absorbed dose, and the ratio of the synovium absorbed dose to the bone absorbed dose. It was found that thermal neutron beams are optimal for treatment. Computation of the dose distribution in the knee requires the simulation of particle transport from the neutron source to the knee phantom through the moderator. A method was developed to predict the dose distribution in the knee from any neutron and photon beam spectra incident on the knee. This method revealed to be reasonably accurate and enabled one to reduce by a factor 10 the particle transport simulation time by modeling the moderator only. It was used to design moderators for BNCS with the D-D and D-T nuclear reactions in the second chapter. Treatment times $>2 \mathrm{~h}$ were obtained with the $\mathrm{D}-\mathrm{D}$ reaction. They could potentially be reduced if the ${ }^{10} \mathrm{~B}$ concentration in the synovium was increased. For D-T neutrons, high therapeutic ratios and treatment times $<5$ min were obtained for neutron yields of $10^{14} \mathrm{n} / \mathrm{s}$. This treatment time makes the D-T reaction attractive for BNCS.

The third part describes the development of high-intensity D-D and D-T neutron generators. The main components of the neutron generator — the ion source, the accelerator and the target - are all housed in a sealed metal container without external pumping. Thick target neutron yield computations have been performed to estimate the neutron yield of titanium and scandium targets. With an average deuteron beam current of $1 \mathrm{~A}$ and an energy of $120 \mathrm{keV}$, a neutron production of about $10^{14} \mathrm{n} / \mathrm{s}$ can be estimated for a tritiated target. In mixed deuteron/triton beam operation, a beam current of $2 \mathrm{~A}$ at $150 \mathrm{keV}$ is required for the same neutron output. Despite this lower neutron production, this mode of operation is advantageous because the target lifetime can be increased from a few hours to more than $1000 \mathrm{~h}$. Experimental findings such as high monatomic ion species fractions in the ion source and ion source operation at low gas pressures will enable us to develop high-intensity, sealed compact neutron generators.

Professor Jasmina Vujic Dissertation Committee Chair 


\section{Contents}

List of Figures vii

List of Tables $\quad$ xiv

1 Introduction 1

1.1 Applications of neutrons . . . . . . . . . . . . . . . . 1

1.2 Boron neutron capture therapy and synovectomy . . . . . . . . . . 2

1.3 RF-driven multicusp ion source . . . . . . . . . . . . 3

1.4 Scope of thesis research . . . . . . . . . . . . . . . 4

2 Important neutron sources and neutron interactions $\quad 6$

2.1 Neutron sources. . . . . . . . . . . . . . . . 6

2.2 Neutron interactions with matter . . . . . . . . . . . 9

2.3 Neutron interactions with tissues . . . . . . . . . . . . . . 14

3 Neutron beam design for shallow and deep-seated brain tumors 19

3.1 Introduction . . . . . . . . . . . . . . . . . . 19

3.2 Figures of merit . . . . . . . . . . . . . . 21

3.3 Treatment characteristics as a function of the neutron beam energy . . . . . 24

3.4 Treatment characteristics as a function of the neutron beam diameter . . . 29

3.5 Summary of results and discussion . . . . . . . . . . . . . 34

4 Design of BSAs for BNCT for the D-D and D-T neutron sources $\quad 37$

4.1 Introduction . . . . . . . . . . . . . . . 37

4.2 Neutron source characterization . . . . . . . . . . . . . 39

4.3 Beam-shaping assembly description . . . . . . . . . . . . . . 39

4.4 Estimation of absorbed doses in the brain . . . . . . . . . . 40

4.4 .1 Tissue composition . . . . . . . . . . . . . . 41

4.4.2 Fluence-to-KERMA conversion factors . . . . . . . . . . . . 42

4.4.3 Comparison of method A with method B . . . . . . . . . . 42

4.5 Methodology for the optimization of the BSA . . . . . . . . . 45

4.5.1 Method based on the multigroup neutron diffusion equations . . . 50

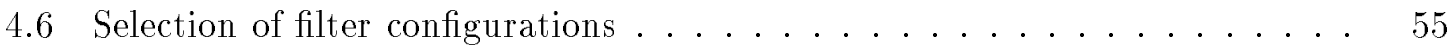

4.7 Moderation of D-D neutrons . . . . . . . . . . . . . . 59 
4.8 Moderation of D-T neutrons . . . . . . . . . . . . . 61

4.8.1 Effect of the BSA diameter on the treatment characteristics . . . . 64

4.8.2 Reflector material and thickness .............. 66

4.8.3 Optimal BSA for D-T neutrons . . . . . . . . . . 68

4.9 Summary of results . . . . . . . . . . . . . . . . . . 72

$5 \quad$ Neutron beam design for boron neutron capture synovectomy $\quad 73$

5.1 Introduction . . . . . . . . . . . . . . . . 73

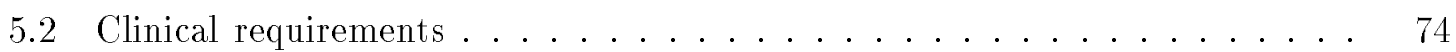

5.3 Dose computations in the knee ................ 76

5.4 Neutron beam dose-response . . . . . . . . . . . . . . . 77

5.4.1 Analysis of the dose-response databases . . . . . . . . . . . 78

5.4.2 Use of dose-response database for the design of BSAs . . . . . 82

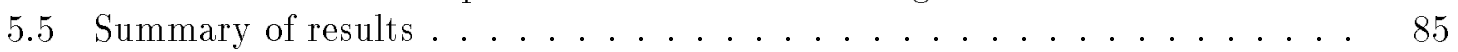

6 Design of BSAs for BNCS for the D-D and D-T neutron sources $\quad 86$

6.1 Neutron source characterization . . . . . . . . . . . . 86

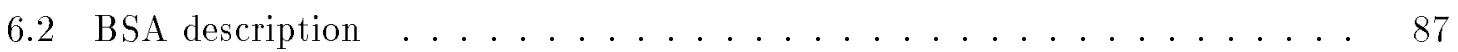

6.3 Neutron beam delimiter . . . . . . . . . . . . . 88

6.3.1 Delimiter thickness and material . . . . . . . . . . 88

6.3.2 Collimator thickness and angle ............... 90

6.4 Methodology for the optimization of the BSAs . . . . . . . . . . 92

6.5 Moderation of D-D neutrons . . . . . . . . . . . . . . . 94

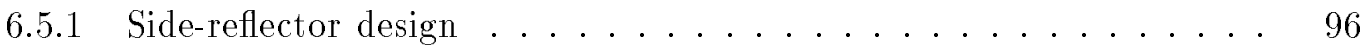

6.5.2 Back-reflector design . . . . . . . . . . . . . . . 97

6.5.3 Optimization of moderator thickness . . . . . . . . . . 98

6.5.4 Optimization of moderator diameter . . . . . . . . . . 99

6.6 Moderation of D-T neutrons . . . . . . . . . . . . . . . 100

6.6 .1 Side-reflector design . . . . . . . . . . . . . . . . . . 102

6.6 .2 Back-reflector design . . . . . . . . . . . . . . . . . . . 104

6.6.3 Optimization of moderator diameter . . . . . . . . . . 105

6.6.4 Optimization of moderator composition and thickness . . . . . 106

6.7 Summary of results . . . . . . . . . . . . . . . 109

7 Compact sealed high-intensity neutron generator $\quad 110$

7.1 Introduction . . . . . . . . . . . . . . . . 110

7.2 Target design . . . . . . . . . . . . . . . . . . . . 112

7.2.1 Thick-target neutron yield computations . . . . . . . . . . . 113

7.2.2 Thick-target neutron yields for monoisotopic ion beams . . . . . . 114

7.2.3 Thick-target neutron yields for mixed ion beams . . . . . . . . 118

7.2.4 Effect of the monatomic/diatomic species fractions in the ion beam . 120

7.2.5 Thermodynamic stability of titanium and scandium targets . . . . 124

7.2 .6 Target lifetime . . . . . . . . . . . . . . . . 125

7.2 .7 Summary of results . . . . . . . . . . . . . . . 126




7.3.1 Description of the prototype ion source . . . . . . . . . . . 127

7.3 .2 Ion beam extraction system . . . . . . . . . . . 130

7.3.3 Ion species measurements . . . . . . . . . . . . . 131

7.3.4 Ion beam current density measurements . . . . . . . . . . 137

7.3.5 Summary of results. . . . . . . . . . . . . . . . . . . 142

7.4 Accelerator column . . . . . . . . . . . . . . . . . . . 143

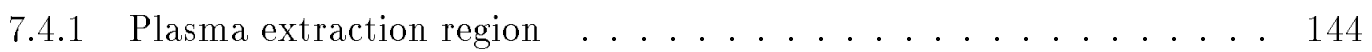

7.4.2 Prototype accelerator column design . . . . . . . . . . . 146

7.4.3 Experimental results . . . . . . . . . . . . . . 153

7.4.4 Design of higher-current accelerator columns . . . . . . . . 155

7.4.5 Summary of results . . . . . . . . . . . . . 156

8 Conclusion $\quad 158$

8.1 Boron neutron capture therapy . . . . . . . . . . . . . 158

8.2 Boron neutron capture synovectomy . . . . . . . . . . . . 159

8.3 Neutron generator . . . . . . . . . . . . . . 160

$\begin{array}{ll}\text { Bibliography } & 162\end{array}$

$\begin{array}{ll}\text { A Glossary } & 168\end{array}$

B Fluence-to-KERMA conversion factors for neutrons, photon mass attenuation coefficients

B.1 Fluence-to-KERMA conversion factors for neutrons in tissues, taken from the International Commission on Radiation Units and Measurements (ICRU) Report No. 46 [14]. . . . . . . . . . . . . . . . . . . 169

B.2 Fluence-to-KERMA conversion factors for neutrons in natural boron, taken from Caswell et al. [16]. . . . . . . . . . . . . . . . 173

B.3 Photon mass attenuation coefficients in tissues, taken from the International Commission on Radiation Units and Measurements (ICRU) Report No. 46 [14].177

B.4 Fluence-to-KERMA conversion factors for neutrons in tissues, taken from Zamenhof et al. [15]. . . . . . . . . . . . . . . 178

C Simulations with the MIRD 5 anthropomorphic model

C.1 MCNP input file corresponding to the MIRD 5 phantom head shown in Fig. 3.1 a and the BSA used in Sec. 4.8.3. . . . . . . . . . 182

C.2 MCNP input file corresponding to the MIRD 5 phantom head shown in Fig. 3.1b and the BSA used in Sec. 4.8.3. . . . . . . . . . . 198 


\section{List of Figures}

2.1 D-D and D-T fusion reaction microscopic cross sections. . . . . . . . 7

2.2 Energy distribution of the neutrons produced by the ${ }^{7} \mathrm{Li}(p, n)^{7}$ Be reaction [10]. 9

2.3 Energy distribution of the neutrons produced by the ${ }^{9} \mathrm{Be}(d, n)^{10} \mathrm{~B}$ reaction [9]. 9

2.4 Fluence-to-KERMA conversion factors for neutrons in tissues [14, 15] and

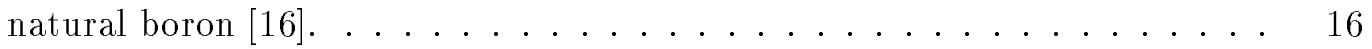

2.5 Photon mass attenuation coefficients in tissues, taken from [14] . . . . . 17

3.1 MIRD-5 phantom head. Ellipsoidal models of the brain, skull, and skin layer. The backbone is shown in the bottom right corner. The monodirectional neutron beam is shown in the upper left corner. In (a) absorbed tumor and healthy-tissue doses are computed as a function of depth within the 2-cmdiam and 0.5 -cm-thick cylinders. In (b) the absorbed doses are computed in cubes of volume $1 \mathrm{~cm}^{3}$. Because of its complexity, model (b) was used only when isodose contour distributions were desired. . . . . . . . . .

3.2 Absorbed doses per neutron emitted by the source versus neutron energy for a 12-cm-diam monodirectional neutron beam. . . . . . . . . . .

3.3 Ratios of absorbed doses versus neutron energy for a 12-cm-diam monodirectional neutron beam. . . . . . . . . . . . . .

3.4 Total absorbed healthy-tissue doses (with their components) corresponding to a 12 -cm-diam, 20-keV monodirectional neutron beam. . . . . . . .

3.5 Total absorbed healthy-tissue doses (with their components) corresponding to a 12 -cm-diam, $7.94-\mathrm{keV}$ monodirectional neutron beam. . . . . . . . .

3.6 Isodose countour plots of the absorbed (a) healthy-tissue and (b) tumor dose distributions for a $12-\mathrm{cm}$-diam neutron beam of energy $7.76 \mathrm{keV}$. . . . . .

3.7 Absorbed doses per neutron emitted by the source for a 6 - $\mathrm{cm}$-diam monodirectional neutron beam. . . . . . . . . . . . . . 30

3.8 Ratios of absorbed doses versus neutron energy for a 6 - $\mathrm{cm}$-diam monodirectional neutron beam. . . . . . . . . . . . . . . .

3.9 Absorbed doses per neutron emitted by the source for a 18-cm-diam monodirectional neutron beam. . . . . . . . . . . . .

3.10 Ratios of absorbed doses versus neutron energy for a 18-cm-diam monodirectional neutron beam. . . . . . . . . . . . . . . 
3.11 Isodose countour plots of the absorbed (a) healthy-tissue and (b) tumor dose distributions for a 6 -cm-diam neutron beam of energy $2.71 \mathrm{keV} \ldots \ldots$. . .

3.12 Isodose countour plots of the absorbed (a) healthy-tissue and (b) tumor dose distributions for an 18-cm-diam neutron beam of energy $7.94 \mathrm{keV}$. . . . .

4.1 BSA showing the 20-cm-diam exit window where the neutron and photon spectra are calculated, referred to as exit window throughout the text. . . .

4.2 Comparison of absorbed healthy-tissue doses computed with method A (shown with errorbars) and method B. Neutrons and photons were not tracked through the delimiter. . . . . . . . . . . . . . .

4.3 Comparison of absorbed tumor doses computed with method A (shown with errorbars) and method B. Neutrons and photons were not tracked through the delimiter. . . . . . . . . . . . . . . . .

4.4 Comparison of absorbed healthy-tissue doses computed with method A with and without the particles transmitted through the delimiter. The dose distributions are normalized to reach a maximum absorbed healthy-tissue dose of 12.5 Gy-equivalent for the distribution neglecting the leakage through the

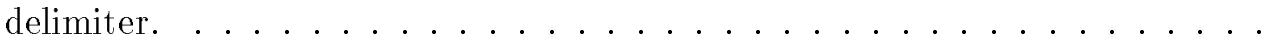

4.5 Optimization for a case where the absorbed skin dose and treatment time constraints are not limiting the maximum achievable tumor dose $\mathrm{D}_{t u}(8 \mathrm{~cm})$. The optimal value of $\mathrm{y}_{i}$ is $70 \mathrm{~cm}$. . . . . . . . . . .

4.6 Optimization for a case similar to the one shown in Fig. 4.5, but where the skin dose curve is different. The maximum tumor dose $\mathrm{D}_{t u}(8 \mathrm{~cm})$ achievable is limited by the skin dose constraint. The optimal value of $\mathrm{y}_{i}$ is $60 \mathrm{~cm}$. . .

4.7 Optimization for a case similar to the one shown in Fig. 4.5, but where the treatment time constraint limits the maximum achievable tumor dose $\mathrm{D}_{t u}(8$ $\mathrm{cm})$. The optimal value of $\mathrm{y}_{i}$ in this case is $50 \mathrm{~cm} . \ldots \ldots \ldots$

4.8 Optimization for a case where none of the two constraints can be satisfied. The optimal $\mathrm{y}_{i}$ in this case is chosen such that the tumor dose $\mathrm{D}_{t u}(8 \mathrm{~cm})$ is maximized. ........................ 49

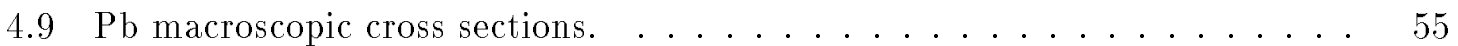

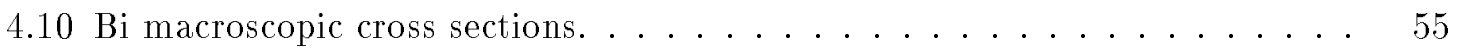

4.11 Fe macroscopic cross sections. . . . . . . . . . . . . . . 56

$4.12{ }^{7} \mathrm{LiF}$ macroscopic cross sections. . . . . . . . . . . . . . . . . . 57

$4.13{ }^{6} \mathrm{LiF}$ macroscopic cross sections. . . . . . . . . . . . . . . 57

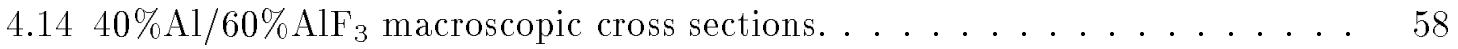

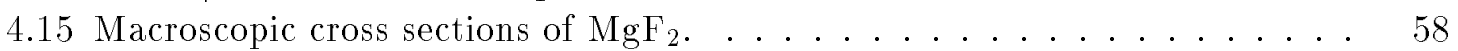

4.16 Neutron energy distribution as a function of ${ }^{7} \mathrm{LiF}$ moderator thicknesses. . . $\quad 59$

4.17 Neutron energy distribution as a function of $40 \% \mathrm{Al} / 60 \% \mathrm{AlF}_{3}$ moderator thicknesses. ........................... 60

4.18 Neutron energy distribution after moderation corresponding to D-D. . . . . 60

4.19 Absorbed healthy-tissue dose (with its components) corresponding to D-D. 61

4.20 Neutron energy distribution as a function of $\mathrm{Pb}$ moderator thicknesses. . . . 62

4.21 Neutron energy distribution as a function of Bi moderator thicknesses. . . . 62

4.22 Neutron energy distribution as a function of Fe moderator thickness. . . . . 63 
4.23 Neutron energy distribution after moderation corresponding to D- $\mathrm{T}$ with a

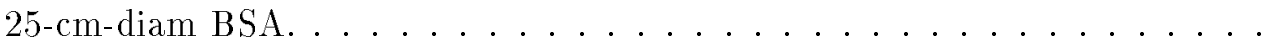

4.24 Neutron energy distribution after moderation corresponding to D- $\mathrm{T}$ with a

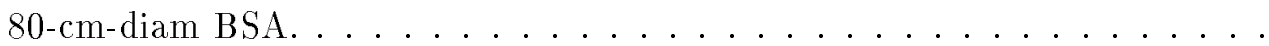

4.25 Treatment time, absorbed tumor dose at $8 \mathrm{~cm}$, and absorbed skin dose as a function of the BSA diameter, accounting for the neutrons and photons transmitted through the delimiter. . . . . . . . . . . . .

4.26 Treatment time, absorbed tumor dose at $8 \mathrm{~cm}$, and absorbed skin dose as a function of the BSA diameter, not accounting for the neutrons and photons transmitted through the delimiter. . . . . . . . . . . . .

4.27 Treatment time versus reflector weight. A neutron source strength of $10^{14}$

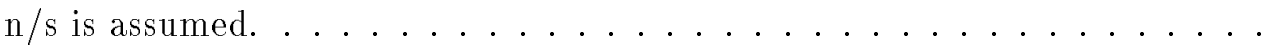

4.28 Absorbed tumor dose at a depth of $8 \mathrm{~cm}$ and absorbed skin dose as a function of the reflector weight. . . . . . . . . . . . .

4.29 Isodose countour plots of the absorbed (a) healthy-tissue and (b) tumor dose distributions for the neutron beam obtained with the optimal BSA. . . . .

4.30 Neutron energy distribution after moderation corresponding to the optimal

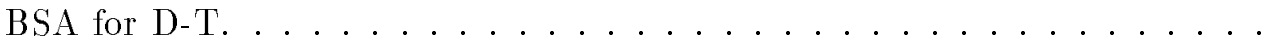

4.31 Absorbed healthy-tissue dose (with its components) corresponding to the optimal BSA for D-T. . . . . . . . . . . . . 70

4.32 Absorbed tumor dose corresponding to the optimal BSA for D-T. . . . . 70

5.1 Cross-section view of the cylindrical knee model used in the MCNP simulations. Absorbed doses are computed as a function of the depth within the 2.5-cm-diam cylinder intersecting the different tissue layers. . . . . . . . .

5.2 Sample beam-shaping assembly (BSA). . . . . . . . . . . . 79

5.3 Therapeutic ratios as a function of the neutron beam energy for isotropic and monodirectional neutron beams. . . . . . . . . . . .

5.4 Absorbed dose rates per unit neutron current at the neutron source as a function of the neutron beam energy for isotropic and monodirectional neutron beams. . . . . . . . . . . . . . . . . .

5.5 Absorbed dose rates versus depth for a 8.7-cm-diam monodirectional neutron beam of energy $0.031 \mathrm{eV}$. A neutron source strength of $7 \times 10^{11} \mathrm{n} / \mathrm{s}$ was used. 81

5.6 Comparison of the dose-response database method with the full simulation method for the computation of the absorbed dose rates. A D-D neutron source of strength $7 \times 10^{11} \mathrm{n} / \mathrm{s}$ was used. . . . . . . . . .

6.1 BSA showing the 8.7-cm-diam exit window where the neutron and photon spectra are calculated, referred to as exit window throughout the text. The collimator is the truncated cone of angle of aperture $\alpha$ located between the

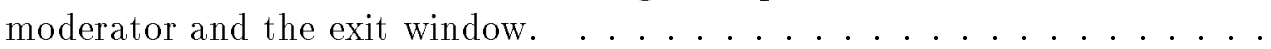


6.2 Delimiter thickness and material parametric study. Ratios of neutron current across the exit window with the delimiter to neutron current across the exit window without delimiter, and neutron current across the exit window to neutron current around the exit window with the delimiter, as a function of the delimiter thickness. . . . . . . . . . . . . . . . .

6.3 Delimiter thickness and material parametric study. Directionality - measured by the ratio of the current $J_{+}$to the flux $\phi$ across the exit window versus delimiter thickness. . . . . . . . . . . . . . .

6.4 Collimator angle and thickness parametric study. Ratio of neutron current across the exit window with the delimiter to neutron current across the exit window without delimiter, as a function of the graphite collimator angle and thickness. The delimiter is composed of a 5-cm-thick graphite layer followed by a 8 -cm-thick lithiated polyethylene layer. . . . . . . . . . . .

6.5 Collimator angle and thickness parametric study. Ratio of neutron current across the exit window to neutron current around the exit window with the delimiter, as a function of the graphite collimator angle and thickness. The delimiter is composed of a 5 -cm-thick graphite layer followed by a 8 -cm-thick lithiated polyethylene layer. . . . . . . . . . . . . . . . . 91

6.6 Macroscopic cross sections of heavy water. . . . . . . . . . . . . . 94

6.7 Comparison of treatment times and therapeutic ratios obtained with the dose-response database and the full simulation methods. . . . . . . . .

6.8 Side-reflector material and thickness parametric study. Therapeutic ratios as a function of the side-reflector material and thickness. . . . . . . . .

6.9 Side-reflector material and thickness parametric study. Treatment time as a function of the side-reflector material and thickness. . . . . . . . . .

6.10 Back-reflector material and thickness parametric study. Therapeutic ratios as a function of the back-reflector material and thickness. . . . . . . . .

6.11 Back-reflector material and thickness parametric study. Treatment time as a function of the back-reflector material and thickness. . . . . . . . . .

6.12 Therapeutic ratios and treatment time as a function of the heavy water moderator thickness. . . . . . . . . . . . . .

6.13 Therapeutic ratios and treatment time as a function of the heavy water moderator diameter. . . . . . . . . . . . . .

6.14 Comparison of treatment times and therapeutic ratios obtained with the dose-response database and the full simulation methods using a lead delimiter.102

6.15 Comparison of treatment times and therapeutic ratios obtained with the dose-response database and the full simulation methods using a graphite

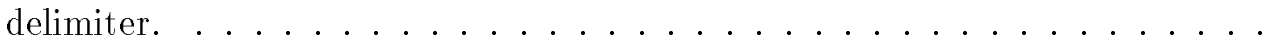

6.16 Side-reflector material and thickness parametric study. Therapeutic ratios as a function of the side-reflector material and thickness. . . . . . . .

6.17 Side-reflector material and thickness parametric study. Treatment time as a function of the side-reflector material and thickness. . . . . . . . . .

6.18 Back-reflector material and thickness parametric study. Therapeutic ratios as a function of the back-reflector material and thickness. . . . . . . . 
6.19 Back-reflector material and thickness parametric study. Treatment time as a function of the back-reflector material and thickness. . . . . . . . .

6.20 Moderator diameter parametric study. Therapeutic ratios and treatment time as a function of the moderator diameter and side-reflector material. A side-reflector thickness of $30 \mathrm{~cm}$ is used for all simulations. . . . . . . .

6.21 Moderator materials thickness parametric study. Therapeutic ratios as a function of moderator material thickness increment. The reference moderator is composed of a 20-cm-thick layer of lead, followed by a 25-cm-thick layer of lithium fluoride ${ }^{7} \mathrm{LiF}$, a 45 -cm-thick layer of heavy water and a 1 -mm-thick layer of lead. . . . . . . . . . . . . . . .

6.22 Moderator materials thickness parametric study. Treatment time as a function of moderator material thickness increment. The reference moderator is composed of a 20-cm-thick layer of lead, followed by a 25-cm-thick layer of lithium fluoride ${ }^{7} \mathrm{LiF}$, a 45 -cm-thick layer of heavy water and a 1 -mm-thick layer of lead. . . . . . . . . . . . . . . . . .

7.1 Schematic diagram of the sealed D-T neutron generator. . . . . . . .

7.2 Neutron yield per unit beam power versus beam energy for deuteron beams impinging on a titanium target loaded with deuterium. $k$ in the units of the energy scale refers to the number of nuclei per ion. . . . . . . . . .

7.3 Neutron yield per unit beam power versus beam energy for deuteron beams impinging on a scandium target loaded with deuterium. $k$ in the units of the energy scale refers to the number of nuclei per ion. . . . . . . . . .

7.4 Neutron yield per unit beam power versus beam energy for deuteron beams impinging on a titanium target loaded with tritium. $k$ in the units of the energy scale refers to the number of nuclei per ion. . . . . . . . . .

7.5 Neutron yield per unit beam power versus beam energy for deuteron beams impinging on a scandium target loaded with tritium. $k$ in the units of the energy scale refers to the number of nuclei per ion. . . . . . . . . .

7.6 Neutron yield per unit beam power versus beam energy for triton beams impinging on a titanium target loaded with deuterium. $k$ in the units of the energy scale refers to the number of nuclei per ion. . . . . . . . . .

7.7 Neutron yield per unit beam power versus beam energy for triton beams impinging on a scandium target loaded with deuterium. $k$ in the units of the energy scale refers to the number of nuclei per ion. . . . . . . . . .

7.8 Neutron yield per unit beam power versus beam energy for mixed $50 \%$ deuteron $/ 50 \%$ triton beams impinging on a titanium target. $k$ in the units of the energy scale refers to the number of nuclei per ion. . . . . . . . .

7.9 Neutron yield per unit beam power versus beam energy for mixed $50 \%$ deuteron $/ 50 \%$ triton beams impinging on a scandium target. $k$ in the units of the energy scale refers to the number of nuclei per ion. . . . . . . .

7.10 Neutron yield per unit beam power versus beam energy for multispecies deuteron beams impinging on a titanium target loaded with deuterium. The atomic ratio is set to $2.0 \ldots \ldots \ldots \ldots \ldots$ 
7.11 Neutron yield per unit beam power versus beam energy for multispecies deuteron beams impinging on a scandium target loaded with deuterium. The atomic ratio is set to $2.0 \ldots \ldots \ldots \ldots$

7.12 Neutron yield per unit beam power versus beam energy for multispecies, mixed $50 \%$ deuteron $/ 50 \%$ triton beams impinging on a titanium target loaded with $50 \%$ deuterium $/ 50 \%$ tritium. The atomic ratio is set to 2.0 . . . . .

7.13 Neutron yield per unit beam power versus beam energy for multispecies, mixed $50 \%$ deuteron $/ 50 \%$ triton beams impinging on a scandium target loaded with $50 \%$ deuterium $/ 50 \%$ tritium. The atomic ratio is set to 2.0 . .

7.14 Ion source with quartz antenna and magnetic filter. . . . . . . . . . . .

7.15 Schematic diagram of the RF-driven ion source. . . . . . . . . .

7.16 Ion source (a) with and (b) without magnetic filter. . . . . . . . . .

7.17 Ion beam extraction system. . . . . . . . . . . . . .

7.18 Matching network and ion source installed on the accelerator column. . . .

7.19 Experimental setup for the ion species measurements from the prototype ion source. . . . . . . . . . . . . . . . . 133

7.20 Sample ion species distribution measured with the mass spectrometer. . . . 134

7.21 Hydrogen ion species fractions versus hydrogen gas pressure, cw RF power

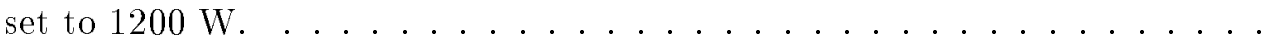

7.22 Hydrogen ion species fractions versus cw RF power for gas pressure of 4 mTorr, with and without magnetic filter. . . . . . . . . .

7.23 Hydrogen ion species distribution obtained with a cw RF power of $1200 \mathrm{~W}$ and a gas pressure of 4 mTorr in the filter-equipped ion source. . . . . . .

7.24 Minimum cw RF power to sustain plasma for different gas pressures. . . . .

7.25 Schematic diagram of the experimental setup used for the current density measurement from the prototype ion source. . . . . . . . . .

7.26 Current density versus extraction voltage for different $\mathrm{cw}$ RF power levels for a filter-equipped ion source and a gas pressure of 4 mTorr. . . . . . .

7.27 Faraday cup and extraction electrode current versus extraction voltage for a filter-equipped ion source, an RF power of $1.5 \mathrm{~kW}$ and a gas pressure of 8 mTorr. . . . . . . . . . . . . . . . . .

7.28 Extracted current density at saturation versus $\mathrm{cw}$ RF power for the ion source without magnetic filter for two gas pressures. . . . . . . . . . . . . . . 142

7.29 Extracted current density at saturation versus RF power for the filter-equipped ion source with antenna coil positioned $5.7 \mathrm{~cm}$ away from the plasma electrode.142

7.30 Extracted current density at saturation versus RF power for the filter-equipped ion source with antenna coil positioned $5.0 \mathrm{~cm}$ away from the plasma electrode. 143

7.31 Menisci corresponding to (a) under-dense, (b) intermediate, and (c) overdense plasma. . . . . . . . . . . . . . . .

7.32 Single hole type accelerator column design using IGUN simulation code for proton beam. The current density at the ion source is $36 \mathrm{~mA} / \mathrm{cm}^{2}$. . . . 147

7.33 Single hole type accelerator column design using IGUN simulation code for proton beam. The current density at the ion source is $389 \mathrm{~mA} / \mathrm{cm}^{2}$. . . . 148

7.34 Mechanical drawing of the ion source and accelerator column. . . . . . . . 148 
7.35 Photograph of the accelerator column. . . . . . . . . . . . . 150

7.36 Schematic diagram of the experimental setup used for testing the high-voltage accelerator column. . . . . . . . . . . . . . . 150

7.37 View of the experimental setup in the test stand. . . . . . . . . . . . 151

7.38 View of the ion source in the test stand. . . . . . . . . . . . . 152

7.39 Photograph of the Faraday cup showing the beam spot and the two magnets for the secondary emission electron suppression. . . . . . . . . . . . 154

7.40 Slit type accelerator column design using IGUN simulation code for proton beam. The current density at the ion source is $10 \mathrm{~mA} / \mathrm{cm}^{2}$, the monatomic and diatomic ion species are assumed to be $85 \%$ and $15 \% \ldots \ldots \ldots$

7.41 Proton beam losses as a function of distance from the plasma electrode and neutral gas pressure. . . . . . . . . . . . . . 156 


\section{List of Tables}

2.1 Data of the "big-4" neutron source reactions [7]. . . . . . . . . 7

2.2 Less common neutron source reactions, taken from (a) Ref. [9] and (b) Ref. [7]. 8

2.3 Properties of neutron-producing reactions, taken from (a) Sec. 7.2.2, (b) Ref. [11], and (c) Ref. [12]. . . . . . . . . . . . . 10

2.4 Properties of common moderator materials. . . . . . . . . . . . . . 12

3.1 Dosimetric properties of monodirectional neutron beams as a function of their

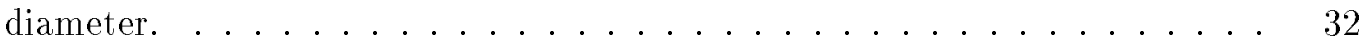

4.1 Elemental compositions in weight percent fractions and densities in grams per cubic centimetre of the skin, bone, and brain used in BNCT_RTPE. . . 42

4.2 Comparison of treatment characteristics for different neutron sources. . . . 71

5.1 Density and elemental composition of bone and soft tissue in weight percentages. . . . . . . . . . . . . . . . . 77 


\section{Acknowledgements}

I would like to thank the members of my dissertation committee Professor J. Vujic, Professor P. Chambré, Professor W. Kunkel and Doctor K.-N. Leung for their scientific advice, help and support during this project. I would also like to express gratitude to all the members of the Plasma and Ion Source Technology Group for their scientific and technical assistance, without whom this project would not have been possible. Thanks should also be given to the team of undergraduate students who helped me complete this research. From left to

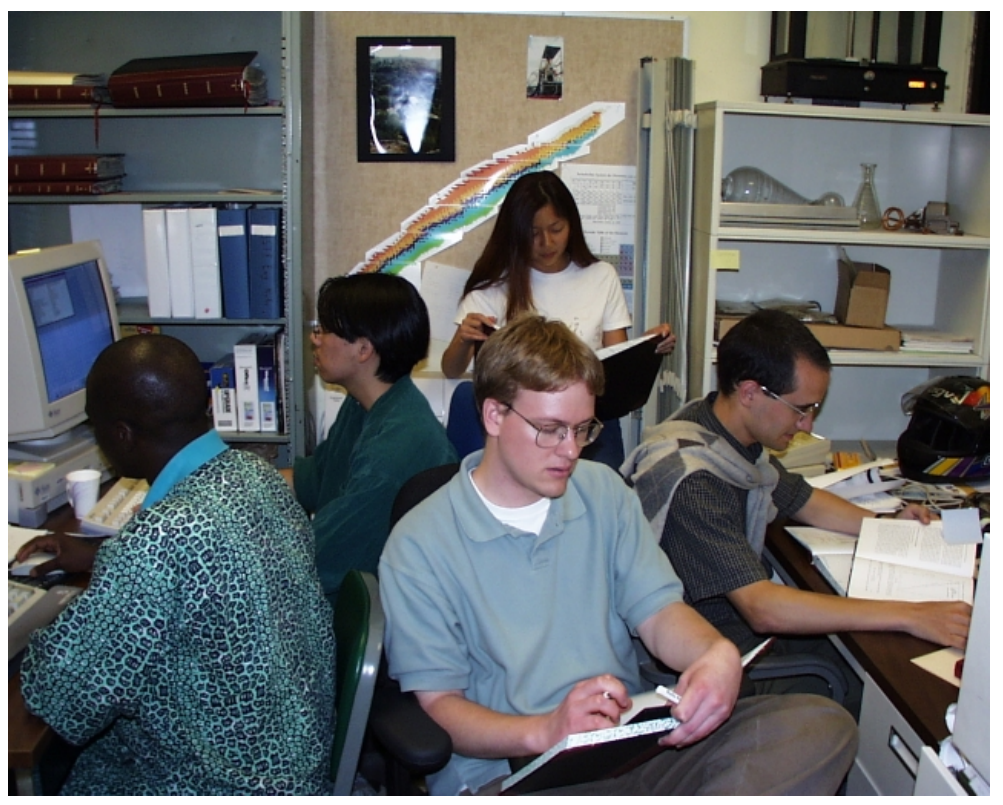

right in the photograph: Bukola Afolayan, Allen Chen, Lydia Wu and Bryan Tolmachoff. The last person on the right is the author of this thesis. This work has been supported by Sandia National Laboratory, the U.S. Department of Energy under contract number DE-AC03-76SF00098, and the Laboratory Directed Research and Development Program of Lawrence Berkeley National Laboratory. 


\section{Chapter 1}

\section{Introduction}

\subsection{Applications of neutrons}

Neutrons were first discovered by Chadwick in 1932 [1]. Almost 70 years after this discovery, neutrons are being used in a variety of applications ranging from medicine to nuclear weapons, spanning over a wide spectrum of sciences and technologies such as biology, material science, explosive detection, fission and fusion, etc. Among the most important uses of neutrons is the generation of electricity in nuclear power plants. Another technology based on neutrons that is widely used is neutron radiography, which enables the non-destructive testing and evaluation of materials. Neutron radiography provides in-depth images of objects, where conventional techniques fail because of the strong absorption of electromagnetic radiation such as $\mathrm{x}$ rays and $\gamma$ rays in matter. An example of neutron radiography applications is the detection of fractures in turbine blades.

Neutron activation analysis is another application being used to measure the oxygen content in steel in metallurgy, as well as the sulfur content in coal to assess its energy yield. Activation analysis is also being used in airports to detect explosives, weapons and drugs in luggage, and to characterize nuclear spent fuel and radioactive waste in general. Equally important are applications in geophysical exploration of oil and minerals. A potential use of this technique currently under investigation is land-mine detection in fields.

The two applications in which we are particularly interested in are boron neutron capture therapy for treatment of brain tumors and boron neutron capture synovectomy for treatment of rheumatoid arthritis. A short review of these two treatments is given in the next section. 
As our understanding of neutrons and their interactions with matter continue to grow, so will the applications. In the future, we may see new applications of neutrons that might become as important as their use for generation of electrical power.

\subsection{Boron neutron capture therapy and synovectomy}

Boron neutron capture therapy (BNCT) is a binary cancer therapy modality which is very appealing due to its potential for selective cell killing [2]. This therapy is being investigated for several types of cancers including glioblastoma multiforme, a highly malignant and therapeutically persistent brain tumor, for which conventional therapies like chemotherapy, surgery, and radiotherapy are not successfull. BNCT is a straightforward concept that was first published by Gordon Locher in 1936 [3]. The minor stable isotope of boron, ${ }^{10} \mathrm{~B}$, had been shown to interact with low-energy (thermal) neutrons to produce highly energetic, short-range disintegration products [4]. Locher reasoned that if the ${ }^{10} \mathrm{~B}$ could be localized in tumor tissues, the short-range disintegration products would provide an effective and selective therapy. The concept was attractive in principle: The neutron component of this therapy by itself would have little effect on tumor or normal tissue; thermal neutrons (E 25 meV) have insufficient energy to damage tissue; the high-linear transfer (LET) radiation released in the tumor cells by the interaction of ${ }^{10} \mathrm{~B}$ with thermal neutrons $\left({ }^{10} \mathrm{~B}+{ }^{1} \mathrm{n} \rightarrow{ }^{11} \mathrm{~B} \rightarrow\right.$ ${ }^{7} \mathrm{Li}+{ }^{4} \mathrm{He}+2.79 \mathrm{MeV}$ ) would be sufficient to kill or sterilize those cells; and the shortrange of the particles produced by the neutron capture reaction $(\leq 10 \mu \mathrm{m})$ should limit the damage to cells containing ${ }^{10} \mathrm{~B}$. BNCT is thus based on a simple concept relying on two components. The preferential targeting of tumor cells with ${ }^{10} \mathrm{~B}$ and subsequent activation with thermal neutrons (delivered to the patient by an external neutron source) to produce a highly localized radiation.

Another very attractive application of this selective cell killing process is boron neutron capture synovectomy (BNCS) for the treatment of rheumatoid arthritis [5]. Because this disease affects 1 to $3 \%$ of the population in the United States, its economic impact is considerable. In BNCS, a compound containing ${ }^{10} \mathrm{~B}$ is concentrated in the inflamed tissues of the diseased joints by direct injection. The joint is then exposed to a neutron beam and the products of the fission reaction ${ }^{10} \mathrm{~B}(n, \alpha)^{7} \mathrm{Li}$ damage the inflamed tissues irreversibly. The advantages of BNCS over other therapies such as surgery are numerous: First it does not require local anesthesia, second it is less costly and time-consuming, and third it does 
not incapacitate the patient for long periods of rehabilitation. Also, one should realize that complete removal of the inflamed tissues by surgery is technically difficult due to the recesses and crevices of the joints.

\section{$1.3 \quad$ RF-driven multicusp ion source}

The neutron output of conventional commercial compact neutron generators is in the order of a few times $10^{8} \mathrm{n} / \mathrm{s}$ for the $\mathrm{D}-\mathrm{T}$ reaction. This neutron yield is not high enough for either BNCT or BNCS. Usually, these neutron generators employ small Penning-type ion sources coupled to a single gap accelerator at the end of which is placed a tritiated target. To achieve higher neutron output, the accelerator column and ion source have to be scaled up. The RF-driven ion source is identified as a potential replacement of the Penning ion source. It can improve the neutron output while keeping the size of the neutron generator small.

The RF-driven multicusp ion source developed at Lawrence Berkeley National Laboratory has found numerous applications ranging from neutral beam injection systems in Tokamak fusion reactors to particle accelerators, proton therapy machines and ion implantation systems. Such sources are simple to operate, have long lifetimes, high gas efficiencies - or more specifically a low source operation pressure with high ionization efficiency — and provide high-density plasmas with almost pure monatomic species yields. These characteristics make the RF-driven ion source an excellent candidate for a compact, high-output, sealed neutron generator.

To achieve high-density plasma and high gas efficiencies, the path length of the ionizing primary electrons inside the plasma chamber should be maximized to ensure a large number of ionizations in the background gas. In RF-driven discharges, an alternating electromagnetic field is generated by an antenna in the discharge volume. As a result of this time-varying field, the electrons undergo cyclical accelerations and decelerations. Ionization takes place over the course of energetic electron collisions with the background gas during these oscillations. Additionally, a magnetic multi-pole confinement scheme is utilized in the multicusp ion source to enhance the path length of the ionizing electrons. Specifically, permanent magnets are arranged around the periphery of the ion source to achieve better magnetic confinement of the electrons. The configuration of the magnetic field is different in the Penning ion sources, in which only axial magnetic field permeates 
the discharge volume. Better primary electron confinement and consequently higher-density plasmas can be obtained with the multicusp RF-driven ion sources. As a result of the higherplasma density, larger beam current density can be extracted, one can therefore reduce the extraction area and the overall size of the ion source. Due to the presence of the magnets around the discharge chamber, a large fraction of the discharge volume in the multicusp source is free of magnetic field, a feature which enables this type of ion sources to generate large areas of uniform plasma. For this reason, one is able to extract ion beams with the same density over large areas, which cannot be done in the Penning ion source. The large area available for beam extraction further reduces the overall size of the ion source. The multicusp RF-driven ion source is therefore believed to be an excellent candidate for the high-output, though compact neutron generator.

\subsection{Scope of thesis research}

BNCT is the subject of the first part of this thesis. For treatment of deep-seated brain tumors, it is generally accepted that epithermal neutron beams of energy $\sim 10 \mathrm{keV}$ are optimal [6]. Very little work has been performed concerning the neutron beam energy required for shallow brain tumors, which are more often encountered than deep-seated ones. In Chapter 3, a method is presented to quantify the neutron beam quality for different tumor depths in terms of therapeutic quantities.

Because accelerator-based neutron sources produce high-energy neutrons, epithermal neutron beams can be produced using well-designed moderators. This can be exploited to achieve neutron energy spectra and dose distributions in the brain superior to reactorbased neutron sources, which produce mainly thermal neutrons. The fusion reactions D-D and $\mathrm{D}-\mathrm{T}$ have not yet been investigated in detail as potential neutron sources for BNCT. This investigation is the subject of Chapter 4 . In order to treat a 8 -cm-deep brain tumor with the same efficiency and in the same time period as other accelerator-based neutron sources, computational results show that a neutron source output of the order of $10^{14} \mathrm{n} / \mathrm{s}$ is required.

In the second part of this thesis, neutron beams are being designed for BNCS. In Chapter 5, we determine the optimal neutron beam energy range for treatment of rheumatoid arthritis using radiation synovectomy. Thermal neutrons appear to be adequate in terms of doses to the synovium versus doses to the healthy tissues. Based on the results 
of this study, neutron moderators are being designed for both D-D and D-T neutrons in Chapter 6. Computational results show that a neutron source output of the order of $10^{14}$ $\mathrm{n} / \mathrm{s}$ is required for a 10 -min treatment time.

The last part of this thesis discusses the different components of the neutron generator. The first one that will be studied is the target. Calculations of neutron yields for titanium and scandium targets under beam bombardment are presented in Sec. 7.2. Neutron yields of the order of $5 \times 10^{8} \mathrm{n} / \mathrm{s}$ from thick deuterated targets, and $10^{11} \mathrm{n} / \mathrm{s}$ from thick tritiated targets are produced with $150 \mathrm{kV}, 1-\mathrm{mA}$ deuteron beams. The projected neutron output of the LBNL neutron generator is $10^{12} \mathrm{n} / \mathrm{s}$ for the D-D reaction and $10^{14}$ $\mathrm{n} / \mathrm{s}$ for the D-T reaction. These neutron yields correspond to ion beam currents of 1 to $2 \mathrm{~A}$, and are adequate for many applications (including BNCT and BNCS) that require compact neutron sources. The suitability of the RF-driven multicusp ion source for this neutron generator is the subject of Sec. 7.3. Section 7.4 describes the research and development of the accelerator column to be used for neutron production.

Since such a high-intensity D-D or D-T neutron generator is not available on the market, its development will be carried out in 4 incremental steps, through which the beam current will be increased from $1 \mathrm{~mA}$ to $15 \mathrm{~mA}, 150 \mathrm{~mA}$ and finally $1.5 \mathrm{~A}$. The first step has been completed. The ion source and accelerator column system have been designed, built, and tested successfully. The innovative design of the accelerator column for the next steps is presented. 


\section{Chapter 2}

\section{Important neutron sources and neutron interactions}

\section{$2.1 \quad$ Neutron sources}

There are many ways to produce neutrons. Californium sources are based on the spontaneous fission of ${ }^{252} \mathrm{Cf}$, which emits a number of fast neutrons. These sources have a half-life of 2.65 years and need to be replaced regularly. One disadvantage is that they cannot be turned on and off. Therefore, these sources require heavy shielding when not used.

Nuclear reactors produce large neutron fluxes. However, they have several disadvantages. Similar to the californium sources, they cannot be turned on and off rapidly for pulsed operation, which is required in fast pulsed neutron activation analysis. Also, nuclear reactors are large and expensive to build, which is not suitable for medical, industrial or field operations.

Accelerator-based neutron sources operate with charged particles, which are accelerated onto a target, where neutron-producing fission or fusion reactions occur. A number of charged-particle-induced reactions are applied to neutron generation. Using lowenergy accelerators, neutrons are generally produced by the "big- 4 " reactions, ${ }^{2} \mathrm{H}(\boldsymbol{d}, n)^{3} \mathrm{He}$,

${ }^{3} \mathrm{H}(d, n){ }^{4} \mathrm{He},{ }^{3} \mathrm{H}(p, n)^{3} \mathrm{He}$ and ${ }^{7} \mathrm{Li}(p, n){ }^{7} \mathrm{Be}[7]$. These four neutron source reactions with their $Q$-values (defined as the energy released by the reaction) are summarized in Table 2.1. For the last two reactions, i.e., ${ }^{3} \mathrm{H}(p, n)^{3} \mathrm{He}$ and ${ }^{7} \mathrm{Li}(p, n)^{7} \mathrm{Be}$, high incident particle energies 


\begin{tabular}{|l|c|}
\hline Reaction & $Q$-value $[\mathrm{MeV}]$ \\
\hline \hline${ }^{2} \mathrm{H}(d, n)^{3} \mathrm{He}$ & +3.270 \\
\hline${ }^{3} \mathrm{H}(d, n)^{4} \mathrm{He}$ & +17.590 \\
\hline${ }^{3} \mathrm{H}(p, n)^{3} \mathrm{He}$ & -0.763 \\
\hline${ }^{7} \mathrm{Li}(p, n){ }^{7} \mathrm{Be}$ & -1.644 \\
\hline
\end{tabular}

Table 2.1: Data of the "big-4" neutron source reactions [7].

of several $\mathrm{MeV}$ are required, because the reactions have laboratory thresholds of 1.019 and $1.881 \mathrm{MeV}$ [8], respectively. Large accelerator facilities are needed to produce the incident particle beam. On the contrary, the deuterium and tritium of the first two fusion reactions do not have to be accelerated to very high energies to generate a significant neutron yield, because the coulomb barrier between the incident deuteron and the light target nucleus is relatively small.

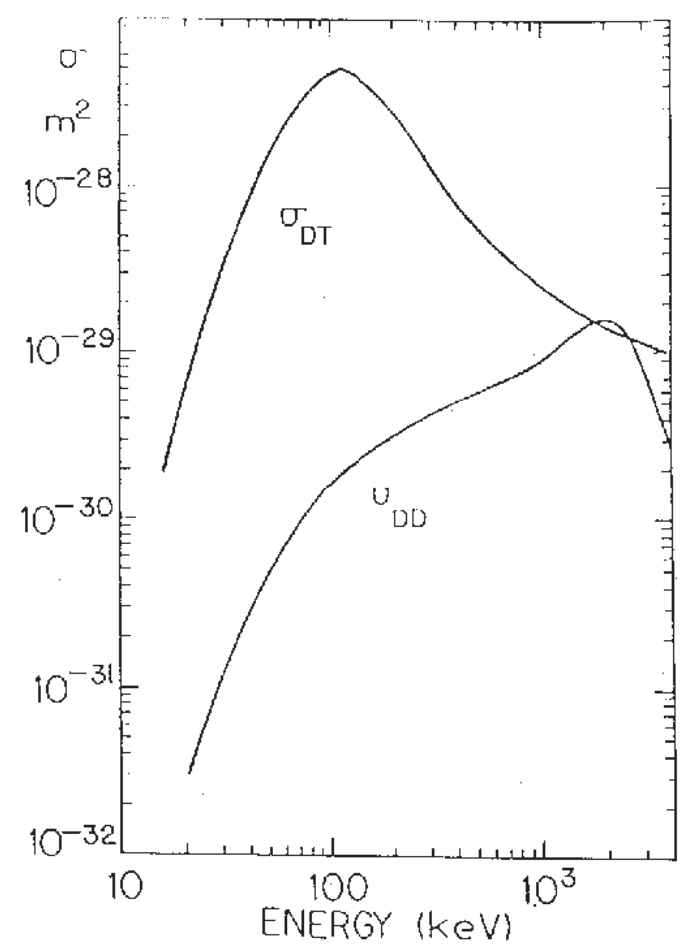

Figure 2.1: D-D and D-T fusion reaction microscopic cross sections.

As shown in Fig. 2.1, the neutron-production cross sections of the D-D and D-T reactions are high at relatively low energies, and an acceleration voltage between 100 and 
$200 \mathrm{kV}$ is enough to produce high neutron yields. The low acceleration voltage requirement makes these reactions viable candidates for compact, portable neutron generators. For oilwell logging applications, for instance, the D-D and D-T tubes are the only neutron sources compact enough to fit within the 2-inch-diam borehole. Since the neutron production cross section of $\mathrm{D}-\mathrm{T}$ peaks around $100 \mathrm{keV}$, the neutron yield will not increase by increasing the deuteron beam energy above $100 \sim 200 \mathrm{keV}$, but only by increasing the deuteron beam current.

In addition to the "big-4" neutron source reactions, there are a number of other possibilities to produce fast neutrons. Typical examples are indicated in Table 2.2.

\begin{tabular}{|l|c|}
\hline Reaction & $Q$-value $[\mathrm{MeV}]$ \\
\hline \hline${ }^{9} \mathrm{Be}(p, n)^{9} \mathrm{~B}(\mathrm{a})$ & -1.85 \\
\hline$\left.{ }^{9} \mathrm{Be}(d, n)\right)^{10} \mathrm{~B}(\mathrm{~b})$ & 4.36 \\
\hline${ }^{7} \mathrm{Li}(d, n)^{8} \mathrm{Be}(\mathrm{b})$ & 15.03 \\
\hline$\left.{ }^{51} \mathrm{~V}(p, n)\right)^{51} \mathrm{Cr}(\mathrm{b})$ & -0.33 \\
\hline
\end{tabular}

Table 2.2: Less common neutron source reactions, taken from (a) Ref. [9] and (b) Ref. [7].

The two applications BNCT and BNCS considered in this thesis require epithermal and thermal neutrons. Therefore, it is of interest to look at the energy distributions of the various neutron sources. The energy distribution of the neutrons produced by the ${ }^{7} \operatorname{Li}(p, n)^{7}$ Be reaction is shown in Fig. 2.2 for various incident proton energies. This figure shows only those neutrons produced in the forward $30^{\circ}$ cone with respect to the proton beam. The nuclear reaction ${ }^{9} \mathrm{Be}(d, n)^{10} \mathrm{~B}$ has recently been proposed for BNCT and BNCS, the energy distribution at $0^{0}$ for a thick and a thin berillium target bombarded by a $1.5-\mathrm{MeV}$ deuteron beam is shown in Fig. 2.3. Concerning the fusion reactions D-D and D-T, all the neutrons produced have about the same energy (near $2.5 \mathrm{MeV}$ for the $\mathrm{D}$ - $\mathrm{D}$ reaction and 14 $\mathrm{MeV}$ for the D-T reaction), because the incident particle energy is small compared with the $Q$-value of either reaction. The neutron energy distributions of beams coming out of reactors depend on the reactor-type. Due to the large amount of moderating materials around the fuel pins, the neutron energy distribution is mainly thermal for most reactor-types.

Properties of several neutron-producing reactions are summarized in Table 2.3 


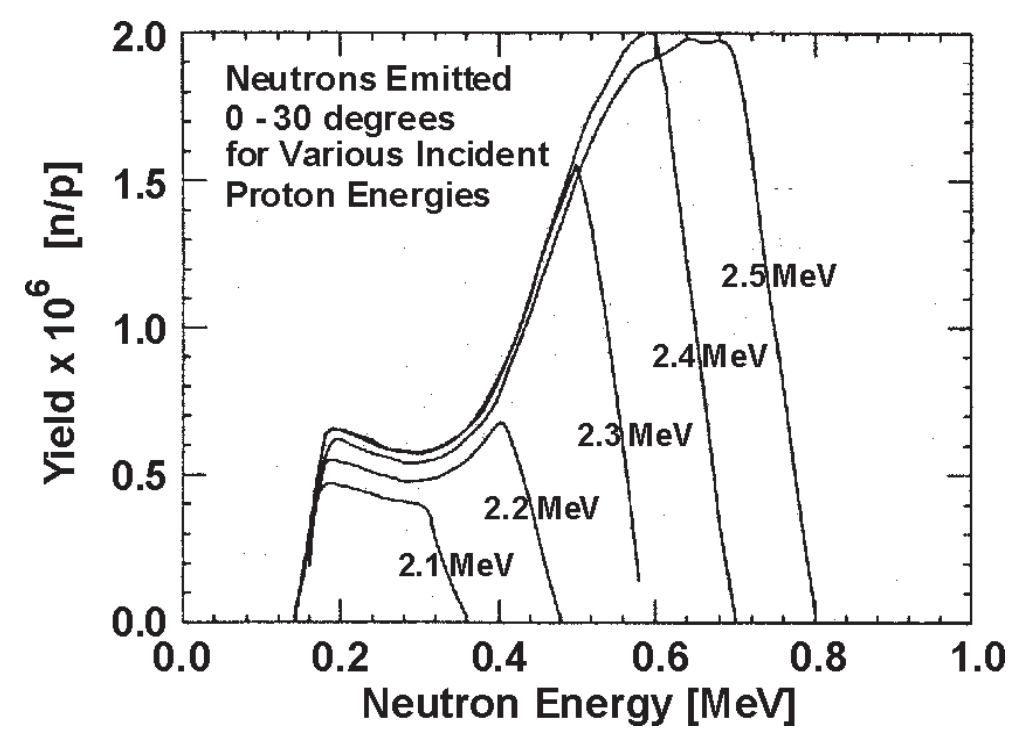

Figure 2.2: Energy distribution of the neutrons produced by the ${ }^{7} \operatorname{Li}(p, n)^{7}$ Be reaction [10].

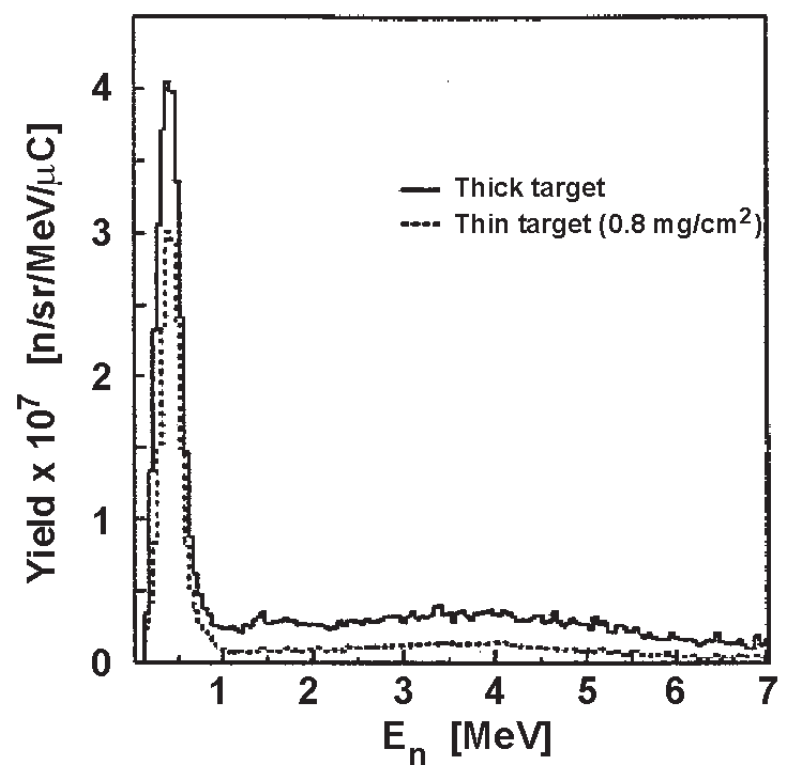

Figure 2.3: Energy distribution of the neutrons produced by the ${ }^{9} \operatorname{Be}(d, n){ }^{10} \mathrm{~B}$ reaction [9].

\subsection{Neutron interactions with matter}

Because neutrons are neutral particles, they do not interact with the electrons in an atom, nor with the positive charge of the nucleus. They pass through the atomic electron cloud and interact directly with the nucleus. Neutrons interact with nuclei in one of the 


\begin{tabular}{|l|c|r|c|}
\hline Reaction & $\begin{array}{c}\text { Ion beam } \\
\text { energy }[\mathrm{MeV}]\end{array}$ & $\begin{array}{r}\text { Neutron yield } \\
{[\mathrm{n} / \mathrm{s} / \mathrm{mA}]}\end{array}$ & $\begin{array}{c}\text { Neutron energy } \\
{[\mathrm{MeV}]}\end{array}$ \\
\hline${ }^{2} \mathrm{H}(d, n)^{3} \mathrm{H}(\mathrm{a})$ & 0.15 & $4.7 \times 10^{8}$ & 2.5 \\
\hline${ }^{3} \mathrm{H}(d, n)^{4} \mathrm{He}(\mathrm{a})$ & 0.12 & $1.0 \times 10^{11}$ & 14.1 \\
\hline${ }^{7} \mathrm{Li}(p, n)^{7} \mathrm{Be}(\mathrm{b})$ & 2.5 & $8.9 \times 10^{11}$ & $0.14-0.78$ \\
\hline${ }^{9} \mathrm{Be}(p, n)^{9} \mathrm{~B}(\mathrm{~b})$ & 4.0 & $5.3 \times 10^{11}$ & $0-2.2$ \\
\hline${ }^{9} \mathrm{Be}(d, n)^{10} \mathrm{~B}(\mathrm{c})$ & 4.0 & $5.1 \times 10^{12}$ & $0.1-7.0$ \\
\hline
\end{tabular}

Table 2.3: Properties of neutron-producing reactions, taken from (a) Sec. 7.2.2, (b) Ref. [11], and (c) Ref. [12].

following ways. (a) Elastic scattering. This type of interaction is similar to the interaction between billiard balls. The neutron strikes the nucleus and is elastically scattered. The nucleus stays in the ground state during the interaction. This interaction is abbreviated by the symbol $(n, n)$. (b) Inelastic scattering. This process is identical to elastic scattering except that the nucleus is left in an excited state, and later decays by the emission of $\gamma$ rays. The symbol for inelastic scattering is (n,n'). (c) Radiative capture. The neutron is captured by the nucleus, and one or several $\gamma$ rays are emitted. This interaction type is denoted as $(n, \gamma)$. (d) Charged-particle reactions. The neutron is absorbed by the nucleus and a charged particle such as $\alpha$ or proton is emitted. The symbols of theses reactions are $(n, \alpha),(n, p)$, etc. (e) Neutron-producing reactions. Denoted as $(n, 2 n),(n, 3 n), \ldots$ these reactions occurs only with energetic neutrons. One neutron is absorbed by the nucleus and two or more neutrons are emitted. (f) Fission. Neutron colliding with certain nuclei may cause the nucleus to split apart, that is, to undergo fission.

Each of these processes is characterized by a cross section $\sigma$, which measures the probability that the interaction will occur when a neutron strikes a target. Cross sections are expressed in units of barns, where 1 barn, abbreviated $b$, is equal to $10^{-24} \mathrm{~cm}^{2}$. The sum of the cross sections for all possible interactions is known as the total cross section $\sigma_{t}$.

$$
\sigma_{t}=\sigma_{s}+\sigma_{i}+\sigma_{\gamma}+\sigma_{f}+\ldots
$$

The product of the atomic density $\mathrm{N}$ of the target material and the cross section $\sigma$ is defined as the macroscopic cross section, and is denoted as $\Sigma$. In particular, $\Sigma_{t}=N \cdot \sigma_{t}$ is the macroscopic total cross section. Macroscopic cross sections have units of $\mathrm{cm}^{-1}$. The macroscopic total cross section gives the probability per unit path length that a neutron will undergo some sort of collision as it moves about in the medium. Greater $\Sigma_{t}$ s result in greater attenuations of neutrons by the target. 
Since elastic scattering is a very important process in neutron moderation, it will be analyzed in more detail than the others. When a neutron is elastically scattered from a nucleus at rest, the nucleus recoils from the site of collision. The kinetic energy of the scattered neutron is therefore smaller than the energy of the incident neutron by an amount equal to the energy acquired by the recoiling nucleus. The energy loss in elastic scattering can be found from the laws of conservation of energy and momentum. Let's consider a neutron of energy $\mathrm{E}$ and mass $\mathrm{M}$ incident on a target nucleus of mass $\mathrm{m}$. After collision, the neutron is scattered at the angle $\theta$ with respect to its initial direction. For a grazing collision, i.e., for $\theta=0$, there is no energy loss. For a head-on collision with a nucleus other than hydrogen, the neutron is scattered directly backwards $(\theta=\pi)$ and suffers the largest possible energy loss. The final energy of the scattered neutron is then given by

$$
E_{\text {min }}^{\prime}=\alpha E
$$

where the collision parameter $\alpha$ is defined by

$$
\alpha=\left(\frac{M-m}{M+m}\right)^{2}
$$

In the particular case of hydrogen, the neutron and hydrogen have the same mass, and the largest possible angle the neutron can be scattered is $\theta=\pi / 2$, in which case $\mathrm{E}_{\text {min }}=0$. Equation 2.2 still holds in this case. The average neutron energy loss $\Delta E$ is given by

$$
\Delta E=\frac{1-\alpha}{2} E
$$

The energy range spanned by neutron slowing down is extremely large, ranging from $\sim 20$ $\mathrm{MeV}$ down to $\sim 5 \mathrm{meV}$. Furthermore, the neutron tends to lose a fraction of its incident energy rather than a fixed amount of energy in elastic scattering. This suggests that it would be more convenient to use the logarithm of the neutron energy E. To this end, the neutron lethargy is defined as

$$
u=\ln \left(\frac{E_{0}}{E}\right)
$$

where $\mathrm{E}_{0}$ is chosen as the neutron source energy for a monoenergetic neutron source. The average lethargy gain $\xi$ of a neutron in a collision with a nucleus of arbitrary mass number can be computed using

$$
\xi=1+\frac{\alpha}{1-\alpha} \ln (\alpha)
$$




\begin{tabular}{|l|c|c|c|c|c|c|}
\hline Moderator & $\xi$ & $\begin{array}{c}\rho \\
{\left[\mathrm{g} / \mathrm{cm}^{3}\right]}\end{array}$ & $\begin{array}{c}\text { \# of collisions } \\
\text { from } 2.5 \mathrm{MeV} \\
\text { to } 25 \mathrm{meV}\end{array}$ & $\begin{array}{c}\text { \# of collisions } \\
\text { from } 14.1 \mathrm{MeV} \\
\text { to } 25 \mathrm{meV}\end{array}$ & $\begin{array}{c}\xi \cdot \Sigma_{s} \\
{\left[\mathrm{~cm}^{-1}\right]}\end{array}$ & $\xi \cdot \Sigma_{s} / \Sigma_{a}$ \\
\hline \hline $\mathrm{H}_{2} \mathrm{O}$ & 0.920 & 1.0 & 21 & 22 & 1.35 & 71 \\
\hline $\mathrm{D}_{2} \mathrm{O}$ & 0.509 & 1.1 & 37 & 40 & 0.176 & 5670 \\
\hline $\mathrm{C}$ & 0.158 & 1.60 & 117 & 128 & 0.060 & 192 \\
\hline $\mathrm{Pb}$ & $9.62 \times 10^{-3}$ & 11.34 & 1915 & 2095 & $3.61 \times 10^{-3}$ & 0.645 \\
\hline
\end{tabular}

Table 2.4: Properties of common moderator materials.

The mean lethargy gain per collision is tabulated for several moderators of interest in Table 2.4. Using the mean lethargy gain $\xi$, one can compute the average number of collisions necessary to thermalize $\left(\mathrm{E}_{t h}=25 \mathrm{meV}\right)$ a neutron of energy $\mathrm{E}_{0}$, it is given by

$$
\langle \#\rangle=\frac{\ln \left(\frac{E_{0}}{E_{t h}}\right)}{\xi} .
$$

$\xi$ is tabulated in Table 2.4. One can see how much more effective low mass-number nuclei are at moderating fast neutrons. We have seen that the number of scattering collisions necessary to slow a neutron to thermal energies is inversely proportional to $\xi$. Better moderators are characterized by large values of $\xi$. However, they must also be characterized by large values of $\Sigma_{s}$, or otherwise the probability of a scattering collision will be too small. Hence a more appropriate measure of the moderating power of a material is the product $\xi \cdot \Sigma_{s}$, defined as

$$
\text { Moderating power }=\xi \cdot \Sigma_{s} \text {. }
$$

However, even this parameter is not sufficient to describe the effectiveness of a moderator, because obviously one also wishes the moderator to be a weak absorber of neutrons. Thus it is customary to choose as a figure of merit the moderating ratio, defined as

$$
\text { Moderating ratio }=\xi \cdot \frac{\Sigma_{s}}{\Sigma_{a}} \text {. }
$$

Both moderating power and moderating ratio for different moderators are listed in Table 2.4. One can observe that light water has a much higher moderating power than heavy water, but its moderating ratio is much lower. In other words, light water thermalizes neutrons more rapidly than heavy water, but it attenuates the thermal neutron flux much faster too, because of its higher absorption cross section for thermal neutrons. This is one reason why heavy water is preferred to light water for neutron moderation. 
With the exception of elastic scattering at low neutron energies, most of the neutron reactions proceed in two steps. The incident neutron, upon striking the target nucleus, first coalesces with it to form a compound nucleus. The compound nucleus then decays in a number of ways. For a target nucleus ${ }^{A} Z$, one would have the following reactions:

$$
\begin{array}{r}
{ }^{A} Z+n \rightarrow{ }^{A+1} Z^{*} \rightarrow \quad{ }^{A} Z+n(\text { elastic scattering }) \\
{ }^{A} Z+n^{\prime}(\text { inelastic scattering }) \\
{ }^{A+1} Z+\gamma(\text { radiative capture }) \\
{ }^{A-1} Z+2 n(n, 2 n \text { reaction })
\end{array}
$$

The cross sections of these interactions that proceed by way of compound formation exhibit maxima at certain energies, called resonances. These resonances are due to the fact that the incident neutron and target nucleus are more likely to combine and form a compound nucleus if the energy of the neutron in such that the compound nucleus is produced in one of its excited states.

In elastic scattering, neutrons are moderated via elastic collisions in which a neutron merely bounces off of a nucleus in a billiard-ball fashion. Inelastic scattering occurs when the neutron has sufficient energy $(\geq 50 \mathrm{keV})$ to place the target nucleus in its first excited state. Therefore, $\sigma_{i}$ is zero up to some threshold energy. Generally speaking, the energy at which the first excited state is found decreases with increasing mass number, and, as a consequence, $\sigma_{i}$ is nonzero over a larger energy region for the heavier nuclei than for the lighter nuclei. In inelastic scattering processes, an appreciable fraction of the incident neutron energy goes into exciting the nucleus into this first excited state. Such scattering is extremely important in heavy mass nuclei, in which slowing down by elastic scattering is negligible. Iron has a large inelastic scattering cross section above $\sim 860 \mathrm{keV}$, which can be used beneficially to decrease the fast component of the neutron flux, to the advantage of the epithermal one.

The $(\mathrm{n}, 2 \mathrm{n})$ reaction occuring in materials such as lead, bismuth and iron can be taken advantage of when high-energy neutrons - such as the ones emitted by the D-T reaction - are available. The $(n, 2 n)$ cross sections for these materials are very high for neutron energies higher than $\sim 10 \mathrm{MeV}$, and the neutrons emitted by the reactions are of much lower energy. These characteristics will be used to enhance the number of neutrons from the D-T neutron source. 
Radiative capture produces $\gamma$ rays that are detrimental to the patient. They do not contribute to the selective cell killing desired in therapies based on boron neutron capture reactions. On the contrary, they deliver a photon dose independent on the location of the boron atoms, which is rather uniform throughout the tissues. Even though the moderating power of heavy water is not as high as the one of light water, it has a much lower radiative capture cross section, which is yet another reason why it will be used preferentially for neutron moderation.

\subsection{Neutron interactions with tissues}

When radiation interacts with a target it produces excited and ionized atoms and molecules as well as a large number of secondary electrons. The secondary electrons can produce additional ionizations and excitations until, finally, the energies of all electrons fall below the threshold necessary for exciting the medium. These changes, which require the direct absorption of energy from the incident radiation by the target, represent the initial

physical perturbations from which subsequent chemical and biological effects evolve. The primary physical quantity used in dosimetry is the dose. It is defined as the energy absorbed per unit mass from any kind of ionizing radiation in any target. The unit of dose, $\mathrm{J} / \mathrm{kg}$, is the gray (Gy).

Fast neutrons lose energy primarily by elastic scattering while slow and thermal neutrons have a high probability of being captured. The two principal capture reactions in tissues are ${ }^{1} \mathrm{H}(\mathrm{n}, \gamma){ }^{2} \mathrm{H}$ and ${ }^{14} \mathrm{~N}(\mathrm{n}, \mathrm{p}){ }^{14} \mathrm{C}$. Slow neutrons are quickly thermalized by the body. The first capture reaction releases a $2.22-\mathrm{MeV}$ gamma ray, which could deposit a fraction of its energy in escaping the body. In contrast, the nitrogen-capture reaction releases an energy of $0.626 \mathrm{MeV}$, which is deposited by the proton and recoil carbon nucleus in the immediate vicinity of the capture site. The resulting dose from the exposure of thermal neutrons can be calculated using the capture cross-sections of the elements in the tissues. Knowing the thermal neutron fluence $\phi$, the atomic density of nitrogen in tissues $N$, its thermal-neutron capture cross-section $\sigma$ and the energy $\mathrm{E}=0.626 \mathrm{MeV}$ deposited by each capture event, the resulting dose deposited in the tissues by the capture event is

$$
D=\frac{\phi N \sigma E}{\rho} \quad[G y]
$$

where $\rho$ is the tissue density. 
The dose from fast neutrons is due almost entirely to the energy transfered to the atomic nuclei in tissue by elastic scattering. A fast neutron loses an average of one-half its energy in a single collision with hydrogen. For other nuclei in tissues, the average energy loss can be computed using Eq. 2.4. The dose due to collisions of fast neutrons with nuclides of tissues can be computed using Eq. 2.10 with $\Delta \mathrm{E}$ instead of E. Fast neutrons deposit most of their energy in tissues by means of collisions with hydrogen. Detailed analysis show that neutrons in the energy range $10 \mathrm{keV}$ to $10 \mathrm{MeV}$ deposit approximatley $5 \%$ to $15 \%$ of their energy by collisions with $\mathrm{O}, \mathrm{C}$ and $\mathrm{N}$ nuclei [13].

A quantity related to dose for indirectly ionizing radiation (photons and neutrons) is the initial kinetic energy of all charged particles liberated by the radiation per unit mass. This quantity has the dimensions of doses and is called KERMA, for Kinetic Energy Released per unit MAss. By definition, KERMA includes energy that may subsequently appear as Bremsstrahlung and Auger-electron energies. Dose and KERMA are identical as long as all of the initial kinetic energy of the recoil charged particles can be considered as being absorbed locally at the interaction site. In most cases however, the dose builds up behind a surface irradiated by a beam of neutral particles to a depth comparable with the range of the secondary charged particles generated, while the KERMA, on the other hand, decreases steadily because of the attenuation of the primary radiation with increasing depth. Because KERMA is a local measure of dose, one can calculate the KERMA at any point in tissues, given the photon and neutron fluences and energy spectra at that point, regardless of the surroundings. It is therefore convenient to describe a given radiation field in a given material in terms of the KERMA in this material.

In this thesis, fluence-to-KERMA conversion factors will be used to convert neutron and photon energy-dependent fluences into KERMAs. The fluence-to-KERMA conversion factor for neutrons used in this thesis are displayed in Fig. 2.4, for both tissues and natural boron. They can also be found in appendices B.1, B.2, and B.4.

Because of the similarity between the notions of KERMA and dose, one uses often the term dose for KERMA. The following equation is used for the transformation of neutron fluence $\phi(E)$ into neutron dose $\mathrm{D}_{n}$ in tissues:

$$
D_{n}=\int k(E) \phi(E) d E
$$

where $\mathrm{k}(\mathrm{E})$ is the energy-dependent fluence-to-KERMA conversion factor for neutrons in tissues. Very often, it is of interest to differentiate the thermal neutron dose $\mathrm{D}_{t h}$ from the 


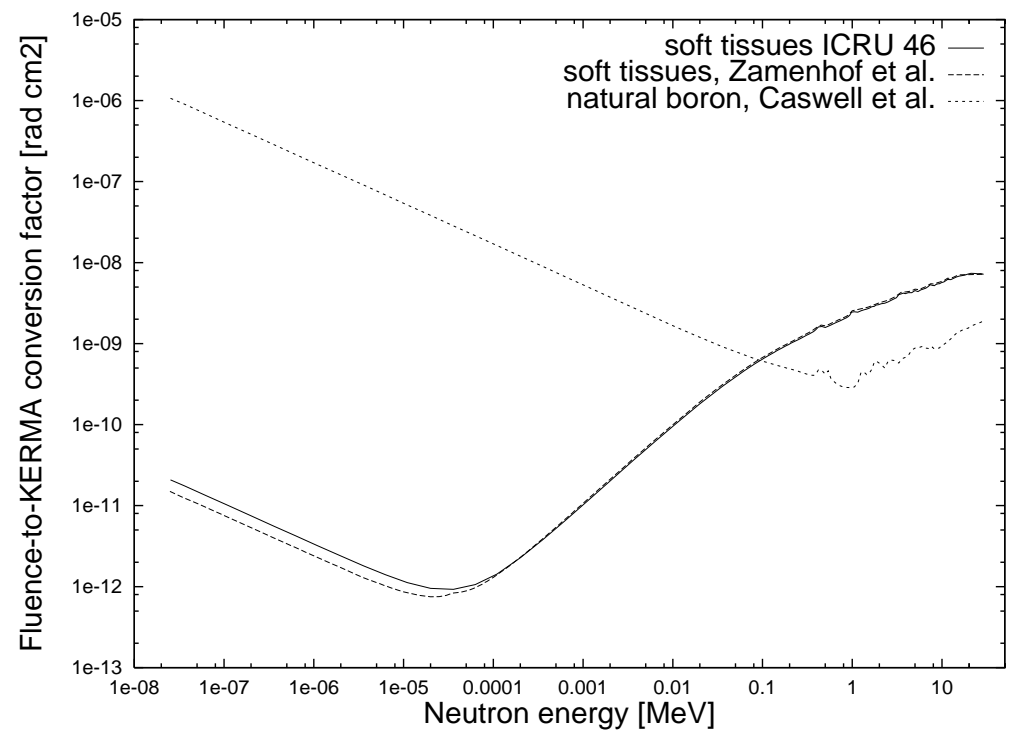

Figure 2.4: Fluence-to-KERMA conversion factors for neutrons in tissues [14, 15] and natural boron [16].

fast neutron dose $\mathrm{D}_{f}$. For that purpose, the cut-off energy between the two energy ranges is set to $0.5 \mathrm{eV}$.

Neutrons produce an additional dose due to the presence of boron in the tissues. This dose is due to the interaction of thermal neutrons with ${ }^{10} \mathrm{~B}$ atoms, inducing the following reaction :

$$
\begin{gathered}
{ }^{10} B+{ }^{1} n \rightarrow{ }^{11} B \rightarrow{ }^{7} \mathrm{Li}+\alpha+\gamma(0.48 \mathrm{MeV}) \\
{ }^{7} \mathrm{Li}+\alpha
\end{gathered}
$$

which has a cross section of 3840 barns for thermal neutrons. The $Q$-value of the reaction is $2.79 \mathrm{MeV}$. With a probability of $93 \%$, a gamma ray of energy $0.48 \mathrm{MeV}$ will be emitted and $2.31 \mathrm{MeV}$ will be shared between the $\alpha$-particle and the ${ }^{7} \mathrm{Li}$ ion. With a probability of $7 \%$, the full energy $2.79 \mathrm{MeV}$ will be shared between the $\alpha$-particle and the ${ }^{7} \mathrm{Li}$ ion. The dosimetric effect of the neutrons due to the presence of boron depend on the concentrations of boron in the tissues. One can compute the boron dose $\mathrm{D}_{B}$ knowing the neutron fluence $\phi(E)$ and the fluence-to-KERMA conversion factors for neutrons in boron using an equation very similar to Eq. 2.11.

For the photon dose, it is computed using the photon mass attenuation coefficients listed in appendix B.3 and displayed in Fig. 2.5. For a photon fluence $\phi(\mathrm{E})$, the photon 


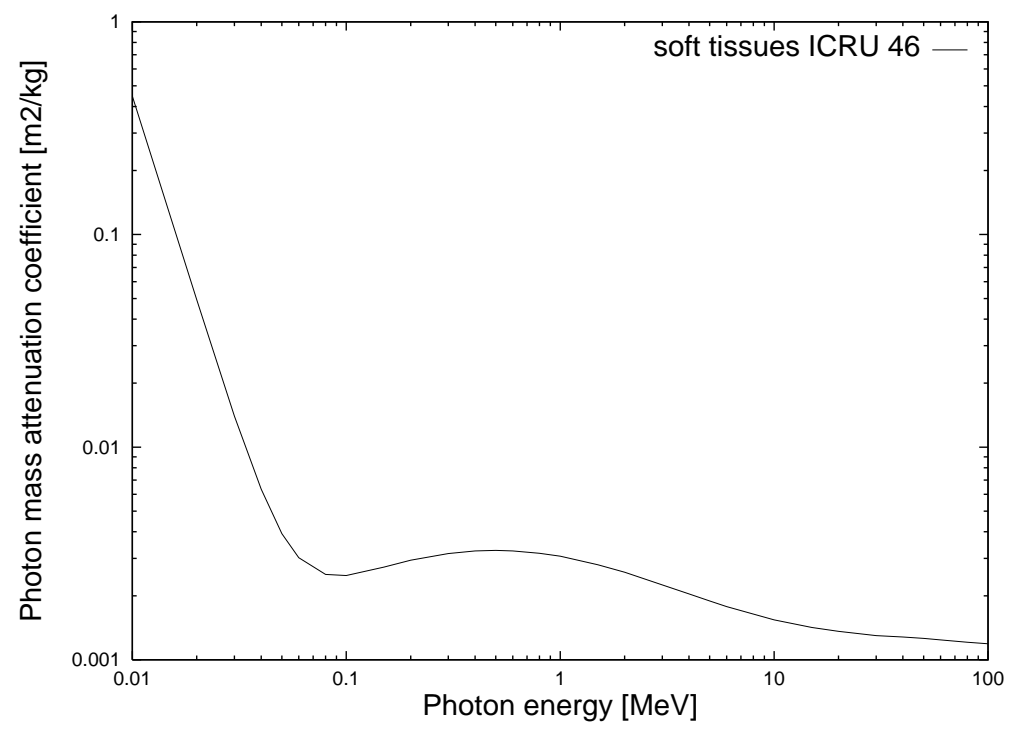

Figure 2.5: Photon mass attenuation coefficients in tissues, taken from [14].

dose $\mathrm{D}_{\gamma}$ is given by

$$
D_{\gamma}=\int k(E) E \phi(E) d E
$$

Dose-response curves depend on the type of radiation used and on the biological endpoint studied. Different radiations can be contrasted in terms of their relative biological effectiveness (RBE) compared with $\mathrm{x}$ rays. If a dose $\mathrm{D}$ of a given type of radiation produces a specific endpoint, then $\mathrm{RBE}$ is defined as the ratio $\mathrm{RBE}=\mathrm{D}_{X} / \mathrm{D}$, where $\mathrm{D}_{X}$ is the $\mathrm{x}$-ray dose needed under the same conditions to produce the same endpoint. The total absorbed tissue doses are obtained by combining the individual dose components $\left(\mathrm{D}_{n}, \mathrm{D}_{B}\right.$ and $\left.\mathrm{D}_{\gamma}\right)$ weighted by their RBE factors, using the following equation:

$$
D_{\text {total }}=R B E_{B} \cdot D_{B}+R B E_{t h} \cdot D_{t h}+R B E_{f} \cdot D_{f}+R B E_{\gamma} \cdot D_{\gamma} \quad[\text { Gy-equivalent }]
$$

The values of the RBE factors depend on the tissues and have to be determined experimentally. Different RBE factors will be used for BNCT and BNCS. For BNCS, the ${ }^{10} \mathrm{~B}$ reaction products $R B E_{B}$ is 4.0 , thermal neutron reaction $R B E_{t h}$ is 3.8 , fast neutron reaction $R B E_{f}$ is 3.8 , and $R B E_{\gamma}$ is 1.0 . These RBE values were taken directly from values used in Ref. [11]. In the case of BNCT, the value of $\mathrm{RBE}_{B}$ depends on the tumor-seeking compound used and will be different whether the compound is located in cancerous cells or not. Therefore, the quantity compound factor $(\mathrm{CF})$ is introduced, it replaces and includes $\mathrm{RBE}_{B}$ in Eq. 2.15. The value of $\mathrm{CF}$ is different in tumor and normal tissue cells. The normal tissue and tumor 
compound factors $C F$ are 1.3 and 3.8 , respectively; the thermal neutron reaction $R B E_{t h}$ is 3.2; the fast neutron reaction $R B E_{f}$ is 3.2 ; and $R B E_{\gamma}$ is 1.0 . The compound factors, and RBE factors are taken from values used in BNL's protocol [17]. 


\section{Chapter 3}

\section{Neutron beam design for shallow and deep-seated brain tumors}

\subsection{Introduction}

Boron neutron capture therapy (BNCT) is a binary cancer therapy modality that is very appealing due to its potential for selective cell killing [2]. This therapy is being investigated for several types of cancers including malignant melanoma [18] and glioblastoma multiforme, a highly malignant and therapeutically persistent brain tumor, for which conventional therapies like chemotherapy, surgery, and radiotherapy are not successful. Another very attractive application of the reaction ${ }^{10} \mathrm{~B}(n, \alpha){ }^{7} \mathrm{Li}$ is boron neutron capture synovectomy (BNCS) [5] for the treatment of rheumatoid arthritis, i.e., inflamed tissues in joints. Since this disease affects 1 to $3 \%$ of the population in the United States, its economic impact is considerable.

BNCT brings together two components. The first component is the delivery of ${ }^{10} \mathrm{~B}$ - a stable isotope of boron with a large cross section for thermal neutron absorption - preferentially to the tumor cells with the help of tumor-seeking compounds. The second component is a beam of low-energy neutrons. When a thermal neutron ( $\mathrm{E} \sim 25 \mathrm{meV})$ is captured by a ${ }^{10} \mathrm{~B}$ atom, the reaction ${ }^{10} \mathrm{~B}(n, \alpha)^{7} \mathrm{Li}$ occurs, releasing a high-energy $\alpha$ particle and ${ }^{7} \mathrm{Li}$ ion. Because of the high linear energy transfer and the high relative biological effectiveness (RBE) of these ions, only cells in close proximity to the reaction are damaged, leaving adjacent cells unaffected. The enhanced uptake of the boron-labeled agent in tumor 
cells versus normal cells results in selective killing of tumor cells, due to the higher dose that can be delivered to them.

Glioblastoma multiforme is characterized by a main tumor mass in the brain accompanied by microscopic fingerlets spreading throughout the surrounding healthy tissues. The ideal neutron beam for irradiation purposes would have to deliver thermal neutrons to the distributed (non-localized) tumor mass and surrounding tissues.

The accepted figures-of-merit used to measure the neutron beam quality are based on biological criteria. The figures-of-merit used in our study are the tumor doses at different depths. These doses must be maximized to increase the probability of cell killing, but they are limited by the Brookhaven National Laboratory's (BNL's) clinical trial protocol [17], which specifies that the local absorbed dose to the healthy tissues must not exceed 12.5 Gy-equivalent anywhere in the brain. BNL's more recent protocols now allow peak doses to substantially exceed this value, but 12.5 Gy-equivalent will be used in this study. No dose limit has been set by BNL's protocol for the skin. Radiation effects in the skin are nonstochastic, and a mild skin reddening, which is not permanent, is observed at doses of approximately 8 Gy-equivalent [19]. Thus, the dose to the skin should be minimized to limit potential carcinogenic effects.

The center of the brain is the most difficult part to reach by the neutrons due to scattering by healthy tissues. To deliver thermal neutrons to the proper area, a previous study [6] showed that one ideally needs to supply neutrons with an energy distribution peaking at around $10 \mathrm{keV}$. The high hydrogen content of the brain slows down the entering epithermal neutrons in such a way that they become thermalized when they reach the desired depth. Neutrons with lower energy contribute less significantly to the dose at the center of the brain because they do not penetrate to this depth. However, they contribute to the dose at shallower depths. Neutrons with energies higher than $40 \mathrm{keV}$ increase the dose to the healthy tissues at the surface of the brain by proton recoil reactions and are thus therapeutically not as useful. This study [6] did not (a) consider shallower tumor sites, which are often encountered; (b) account for the skin dose, which should be limited to minimize potential carcinogenic effects; and (c) go above $800 \mathrm{keV}$, which is lower than the energies of the D-D and D-T neutron sources.

In this Chapter, we present a study to determine which neutron energy is most suitable for treatment of shallow and deep-seated brain tumors. For the purpose of this ideal neutron beam study, we adopted an entrance dose limit to the skin of 8 Gy-equivalent. The 
energy range considered for the neutron beams extends to $14 \mathrm{MeV}$. The Monte-Carlo code MCNP [20] is used for the neutron transport calculations in the phantom head.

\section{$3.2 \quad$ Figures of merit}

In order to optimize the neutron energy for treatment of a tumor at a given depth, we use two figures-of-merit: the absorbed tumor dose to the brain at this depth $\left(\mathrm{D}_{t u}\right)$ and the absorbed dose to the skin $\left(\mathrm{D}_{s k}\right)$ per neutron emitted by the source. With these two quantities, we investigate the dosimetric properties of neutrons up to $14 \mathrm{MeV}$. The optimal neutron beam should maximize the tumor dose, under the constraints that (a) the skin dose does not exceed 8 Gy-equivalent, and (b) the maximum healthy-tissue dose is set to 12.5 Gy-equivalent.

Let $\mathrm{N}$ be the number of source neutrons required to reach the 12.5 Gy-equivalent dose limit on the healthy tissues. The skin and tumor doses are then given by $\mathrm{N} \cdot \mathrm{D}_{s k}$ and $\mathrm{N} \cdot \mathrm{D}_{t u}$, respectively. In mathematical terms, the neutron beam energy $\mathrm{E}_{\text {optimal }}$ is optimal for treatment of a tumor at a depth of $\mathrm{x} \mathrm{cm}$ when

$$
D_{t u}\left(x, E_{\text {optimal }}\right)=\max \left(D_{t u}(x, E)\right)
$$

for $0 \leq E \leq 14 \mathrm{MeV}$, under the constraints

$$
N \cdot D_{\text {sk }}\left(E_{\text {optimal }}\right) \leq 8 \quad G y-\text { equivalent }
$$

and

$$
N \cdot M D_{\text {ti }}\left(E_{\text {optimal }}\right)=12.5 \quad \text { Gy-equivalent }
$$

where $\mathrm{MD}_{t i}(\mathrm{E})$ is defined by

$$
M D_{t i}(E)=\max \left(D_{t i}(\vec{x}, E)\right)
$$

for all the points $\vec{x}$ inside the brain. The number of neutrons $\mathrm{N}$ is determined using Eq. 3.3.

Since the dose limit on the healthy tissues is subject to change as the BNL's clinical trials are pursued, we will often use the ratios $\mathrm{D}_{t u}(\mathrm{x}, \mathrm{E}) / \mathrm{MD}_{t i}(\mathrm{E})$ and $\mathrm{D}_{s k}(\mathrm{E}) / \mathrm{MD}_{t i}$ (E) in the optimization process. These two ratios are the absorbed tumor and skin doses normalized to the dose limit on the healthy tissues. They are not dependent on the healthytissue dose limit. For the optimal energy $\mathrm{E}_{\text {optimal }}$, we have

$$
D_{t u}\left(x, E_{\text {optimal }}\right) / M D_{t i}\left(E_{\text {optimal }}\right)=\max \left(D_{t u}(x, E) / M D_{t i}\left(E_{\text {optimal }}\right)\right)
$$


for $0 \leq E \leq 14 \mathrm{MeV}$, under the constraint

$$
D_{s k}(E) / M D_{t i}\left(E_{\text {optimal }}\right) \leq 8 / 12.5=0.64
$$

Incidentally, knowing the neutron source strength $S$ in $n / s$, we can compute the time $\mathrm{T}$ required for treatment using

$$
T=\frac{N}{S}[s]
$$

This equation will be used extensively in Chapter 4 .

Using the ellipsoidal head of the MIRD-5 anthropomorphic phantom [21], an MCNP [20] simulation study is carried out for monoenergetic and monodirectional beams. Although this study is not directly applicable to realistic neutron beams, it can provide us

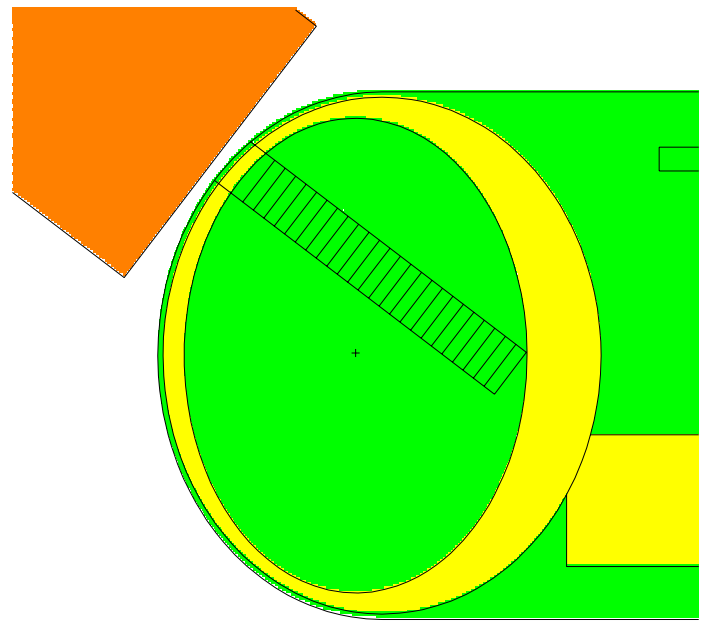

(a)

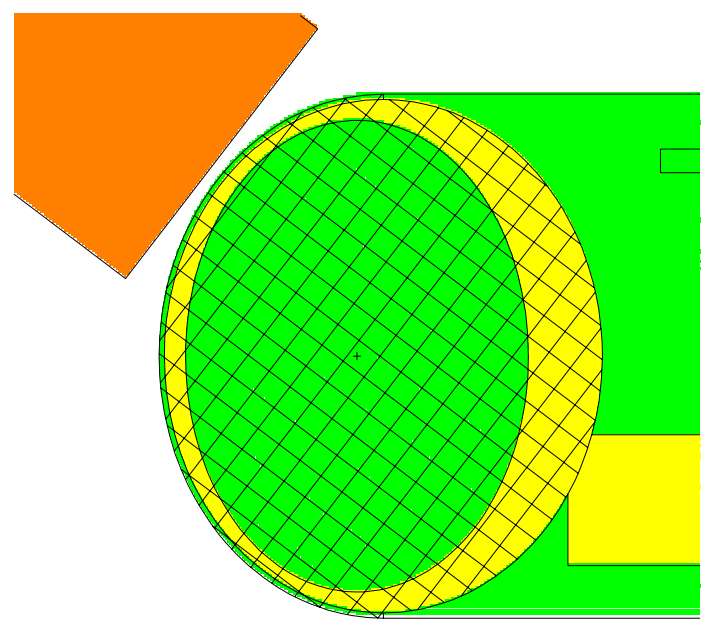

(b)

Figure 3.1: MIRD-5 phantom head. Ellipsoidal models of the brain, skull, and skin layer. The backbone is shown in the bottom right corner. The monodirectional neutron beam is shown in the upper left corner. In (a) absorbed tumor and healthy-tissue doses are computed as a function of depth within the 2-cm-diam and 0.5-cm-thick cylinders. In (b) the absorbed doses are computed in cubes of volume $1 \mathrm{~cm}^{3}$. Because of its complexity, model (b) was used only when isodose contour distributions were desired.

with useful insight and guidance in the design of beam-shaping assemblies (BSA).

The neutron beam used for this simulation study has a diameter of $12 \mathrm{~cm}$, an angle of $37.35 \mathrm{deg}$ with the axis of the MIRD-5 phantom backbone in the sagittal plane, and it is normal to the upper forehead of the phantom. The position of the neutron beam is such that the curvature of the skin at this location is minimum. It guarantees that the 
minimum travel distance from any neutron in the beam to the tumor site at a depth of 8 $\mathrm{cm}$ is at least $8 \mathrm{~cm}$. The monodirectionality of the beam simulates the most penetrating beam achievable. $\mathrm{A}{ }^{10} \mathrm{~B}$ concentration of $10 \mu \mathrm{g}$ per $\mathrm{g}$ is used for the neutron transport in the soft tissues. The $S(\alpha, \beta)$ treatment is used for the thermal neutron treatment. The neutron and photon fluences computed by MCNP [20] in the 2-cm-diam and 0.5-cm-thick cylinders (see Fig. 3.1a) or in the cubes of volume $1 \mathrm{~cm}^{3}$ (see Fig. 3.1b) are modified by the fluence-to-KERMA conversion factors (see Fig. 2.4 and appendix B.1) and the photon mass attenuation coefficients (see Fig. 2.5 and appendix B.3) of the International Commission on Radiation Units and Measurements (ICRU) Report No. 46 [14] to compute the neutron and photon doses. Since the MCNP code provides fluences per neutron emitted by the source, all doses are computed per neutron emitted by the source. All reactions happening at neutron energies less than $0.5 \mathrm{eV}$ (mostly nitrogen absorption reactions) contribute to the thermal neutron dose $D_{t h}$, while all reactions above $0.5 \mathrm{eV}$ (mostly proton recoil reactions) contribute to the fast neutron dose $D_{f}$. The dosimetric effect of neutrons depends on the concentrations of ${ }^{10} \mathrm{~B}$ in both tumor and healthy tissue. To estimate the ${ }^{10} \mathrm{~B}(n, \alpha){ }^{7} \mathrm{Li}$ contribution to the dose, the neutron fluence is modified by ${ }^{10} \mathrm{~B}$ fluence-to-KERMA conversion factors (see Fig. 2.4 and appendix B.2), listed in Caswell et al. [16], and then multiplied by either a factor of $13 \mathrm{ppm}$ (to represent the $13 \mu \mathrm{g} / \mathrm{g}$ of ${ }^{10} \mathrm{~B}$ in healthy tissues) or a factor of 45.5 $\mathrm{ppm}$ (to represent the $45.5 \mu \mathrm{g} / \mathrm{g}$ of ${ }^{10} \mathrm{~B}$ in a tumor). The individual dose components are combined to yield estimates of total absorbed doses per neutron emitted by the source to healthy tissue and tumor, using the following equation:

$$
D_{\text {total }}=C F \cdot D_{B}+R B E_{t h} \cdot D_{t h}+R B E_{f} \cdot D_{f}+R B E_{\gamma} \cdot D_{\gamma} \quad[G y-\text { equivalent } / n]
$$

where the following assumptions are made. The normal tissue and tumor compound factors $(C F)$ are 1.3 and 3.8 , respectively; the thermal neutron reaction $R B E_{t h}$ is 3.2 ; the fast neutron reaction $R B E_{f}$ is 3.2 ; and $R B E_{\gamma}$ is 1.0 . The ${ }^{10} \mathrm{~B}$ concentrations, compound factors, and RBE factors are taken from values used in BNL's protocol [17]. Knowing the distribution of the total absorbed dose to the healthy tissues per neutron emitted by the source, the number of source neutrons required to reach the maximum dose limit of 12.5 Gy-equivalent for the healthy tissues is calculated. Unless specified otherwise in the text, this number is used to scale all the dose distributions shown in this dissertation. Given the neutron source strength, the treatment time can then be computed.

Based on the model shown in Fig. 3.1b, it is found that the maximum healthy- 
tissue dose always occured on the axis of the neutron beam, even though the beam axis did not coincide with the axis of symmetry of the phantom head. The simplified model shown in Fig. 3.1a, with the 2-cm-diam cylindrical tally regions along the beam axis, was then used for most calculations, because it gave a better resolution along the axis of the neutron beam without excessive computational times. To obtain an average relative error of $1 \%$ on the total healthy-tissue dose, one Monte-Carlo simulation took about $220 \mathrm{~min}$ using the model shown in Fig. 3.1a, while it took 680 min using the model shown in Fig. 3.1b.

\subsection{Treatment characteristics as a function of the neutron beam energy}

Figure 3.2 shows the absorbed tumor doses $\left(\mathrm{D}_{t u}\right)$ at $2,4,6$ and $8 \mathrm{~cm}$, the maximum absorbed tissue dose $\left(\mathrm{MD}_{t i}\right)$, and the absorbed skin dose $\left(\mathrm{D}_{s k}\right)$ per neutron emitted by the source, as a function of neutron energy for monoenergetic and monodirectional

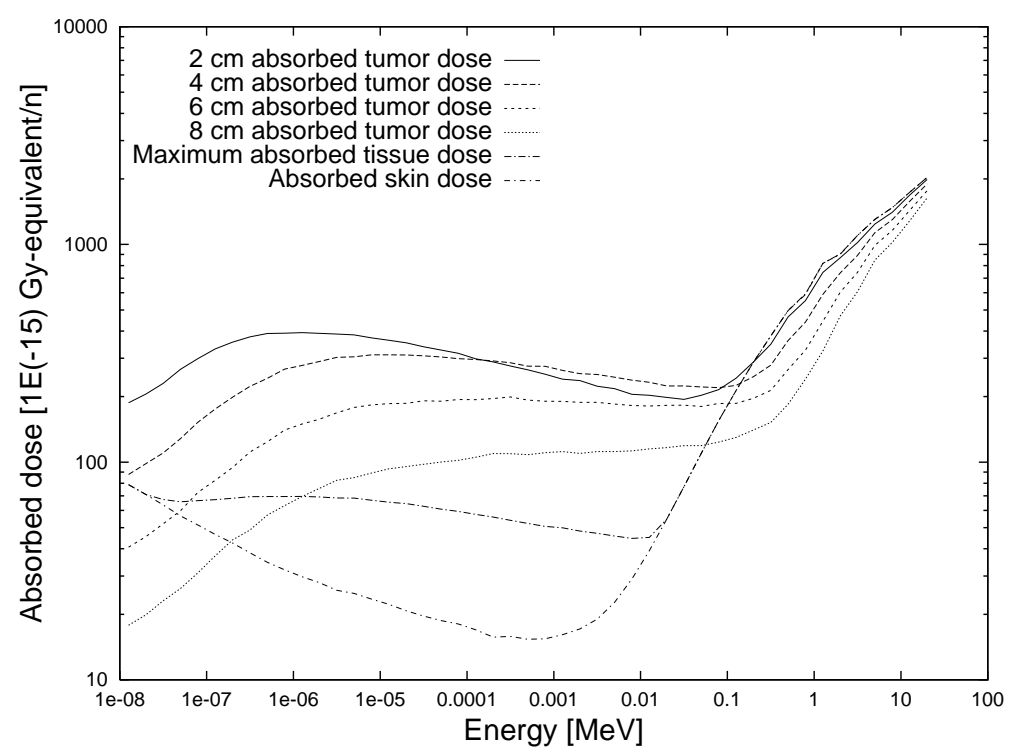

Figure 3.2: Absorbed doses per neutron emitted by the source versus neutron energy for a 12-cm-diam monodirectional neutron beam.

beams. Figure 3.3 shows the ratios of the absorbed tumor doses to the maximum absorbed healthy-tissue dose $\left(\mathrm{D}_{t u} / \mathrm{MD}_{t i}\right)$ - sometimes called therapeutic gains - and the ratio of the absorbed skin dose to the maximum absorbed healthy-tissue dose $\left(\mathrm{D}_{s k} / \mathrm{MD}_{t i}\right)$ as a function of the neutron energy. 
The ratio $\mathrm{D}_{s k} / \mathrm{MD}_{t i}$ increases as the neutron energy increases. For neutrons of energy $20 \mathrm{keV}$ or higher, $\mathrm{D}_{s k}$ is equal to $\mathrm{MD}_{t i}$, indicating that the absorbed healthy-tissue dose is maximum at the skin and decreases monotonically with the depth in the brain (see Fig. 3.4). The spatial distribution of the dose in this high-energy range is predominantly

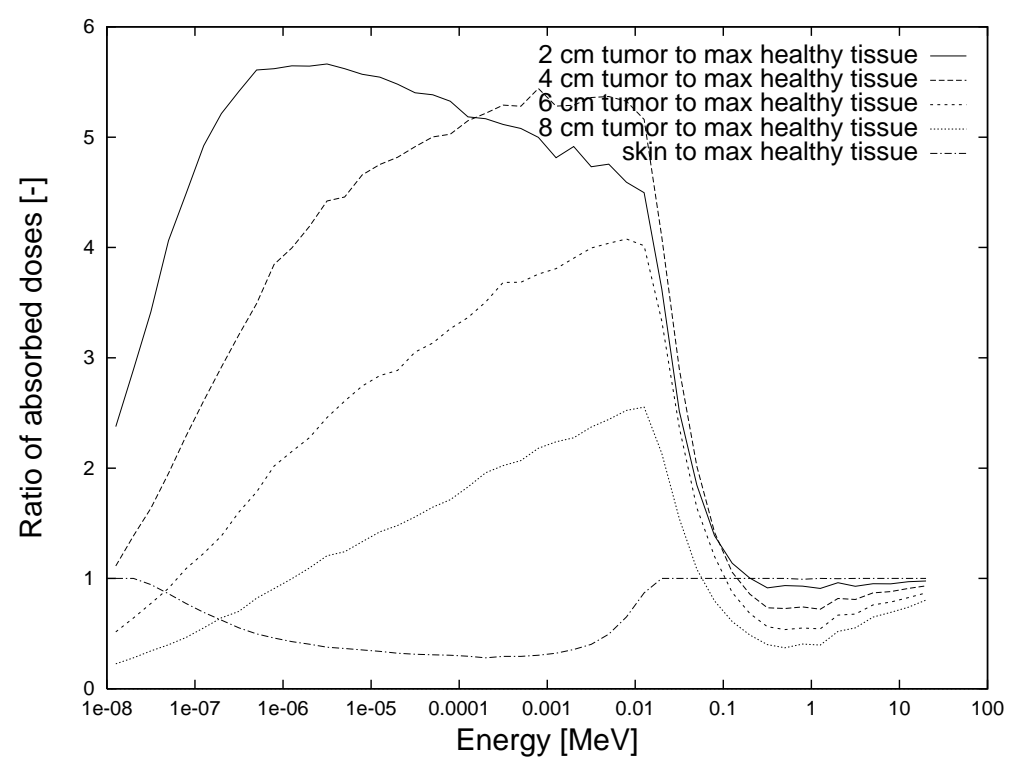

Figure 3.3: Ratios of absorbed doses versus neutron energy for a 12-cm-diam monodirectional neutron beam.

dictated by the proton recoil reactions. Since the absorbed dose to the healthy tissue is limited to 12.5 Gy-equivalent, the increase in fast neutron dose results in the decrease in boron dose in the entire brain. This decrease results in an overall decrease in the absorbed tumor dose (see Eq. 3.8). Figures 3.4 and 3.5, corresponding to 20.0 and $7.94 \mathrm{keV}$ neutron beams, illustrate the reduction in boron dose due to the increase in fast neutron dose.

In summary, high-energy neutron beams (a) generate predominantly proton recoil reactions, resulting in high skin doses and (b) do not take as much advantage of the boron-loaded tumor cells as lower-energy neutron beams. This is one reason why the neutron energy range above $\sim 20 \mathrm{keV}$ is therapeutically undesirable. Concerning lower energy neutron beams, it has been observed that for all neutrons in the energy range $10 \mathrm{eV}$ to 15 $\mathrm{keV}$, the absorbed healthy-tissue dose exhibits a very localized maximum dose between 2.5 and $3.5 \mathrm{~cm}$ (see Fig. 3.5, for example) and decreases toward the skin. Figure 3.3 shows that the ratio $\mathrm{D}_{s k} / \mathrm{MD}_{t i}$ decreases from 1.0 at $20 \mathrm{keV}$ down to about 0.28 at around $0.2 \mathrm{keV}$ and increases slowly back to 1.0 for thermal neutrons at $0.02 \mathrm{eV}$. If we limit the absorbed 


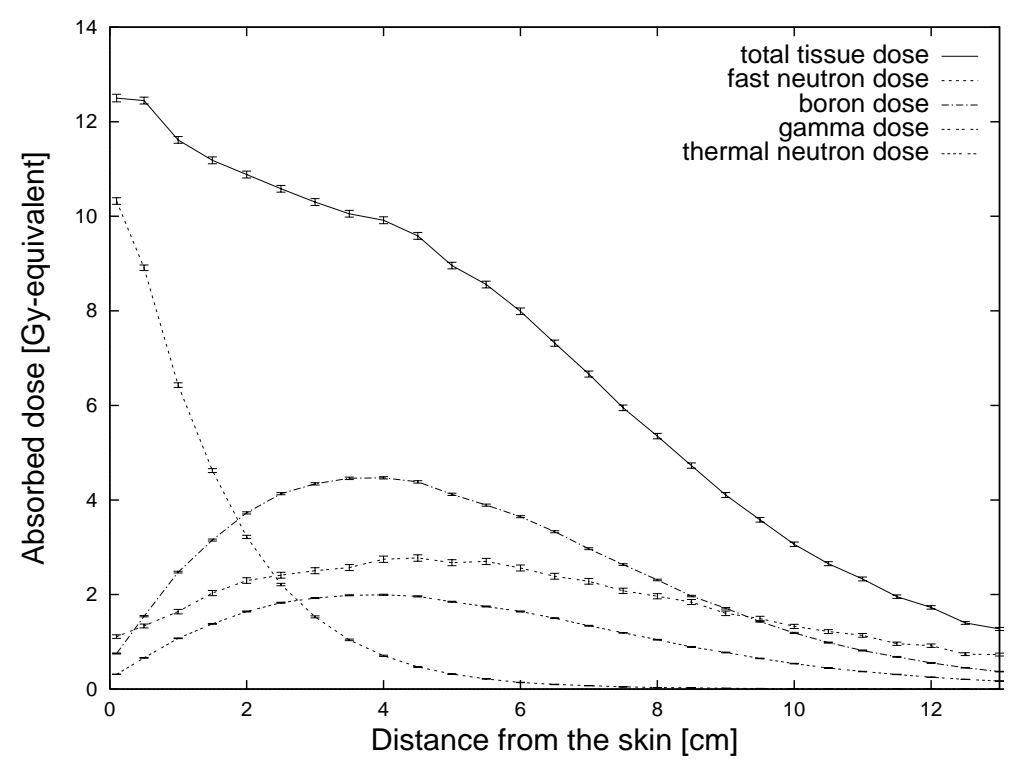

Figure 3.4: Total absorbed healthy-tissue doses (with their components) corresponding to a 12-cm-diam, 20-keV monodirectional neutron beam.

skin dose to 8 Gy-equivalent [19], the ratio $\mathrm{D}_{s k} / \mathrm{MD}_{t i}$ should not exceed $8 / 12.5=0.64$ (see Eq. 3.6). This dose limit imposes neutron energies in the range $0.18 \mathrm{eV}$ to $7.76 \mathrm{keV}$.

In the case of the therapeutic gains, the ratio $\mathrm{D}_{t u}(8 \mathrm{~cm}) / \mathrm{MD}_{t i}$ has a maximum at around $12.6 \mathrm{keV}$ (equal to 2.55). It decreases slowly for lower neutron energies, rapidly for higher neutron energies, and becomes less than unity below $1.28 \mathrm{eV}$ and above $58 \mathrm{keV}$. Neutrons with energies below $1.28 \mathrm{eV}$ and above $58 \mathrm{keV}$ contribute more to the maximum healthy-tissue dose than to the tumor dose at $8 \mathrm{~cm}$. The optimal neutron energy, as defined by Eqs. 3.5 and 3.6, is $7.76 \mathrm{keV}$. This neutron energy maximizes the tumor dose at $8 \mathrm{~cm}$ and results in a skin dose lower than 8 Gy-equivalent. The therapentic gain at $8 \mathrm{~cm}$ for this neutron energy is slightly lower, i.e., 2.52. Compared to the therapeutic gain of 1.37 that we would obtain for a $10-\mathrm{eV}$ neutron beam, for example, the therapeutic gain increase is $120 \%$. The curve representing the ratio $\mathrm{D}_{t u}(6 \mathrm{~cm}) / \mathrm{MD}_{t i}$ shows a similar behavior. It is maximal at $7.94 \mathrm{keV}$. The therapeutic gain at $6 \mathrm{~cm}$ for a $7.76 \mathrm{keV}$ neutron beam is 4.07 , which is $46 \%$ higher than for a $10-\mathrm{eV}$ neutron beam (2.79).

It can be concluded that $7.76-\mathrm{keV}$ neutron beams are optimal for tumors in the depth range 6 to $8 \mathrm{~cm}$. They fulfill both Eqs. 3.5 and 3.6, i.e., they maximize the therapeutic gains within the skin dose limit constraints and are thus ideal for treatment of tumors in that depth range. 


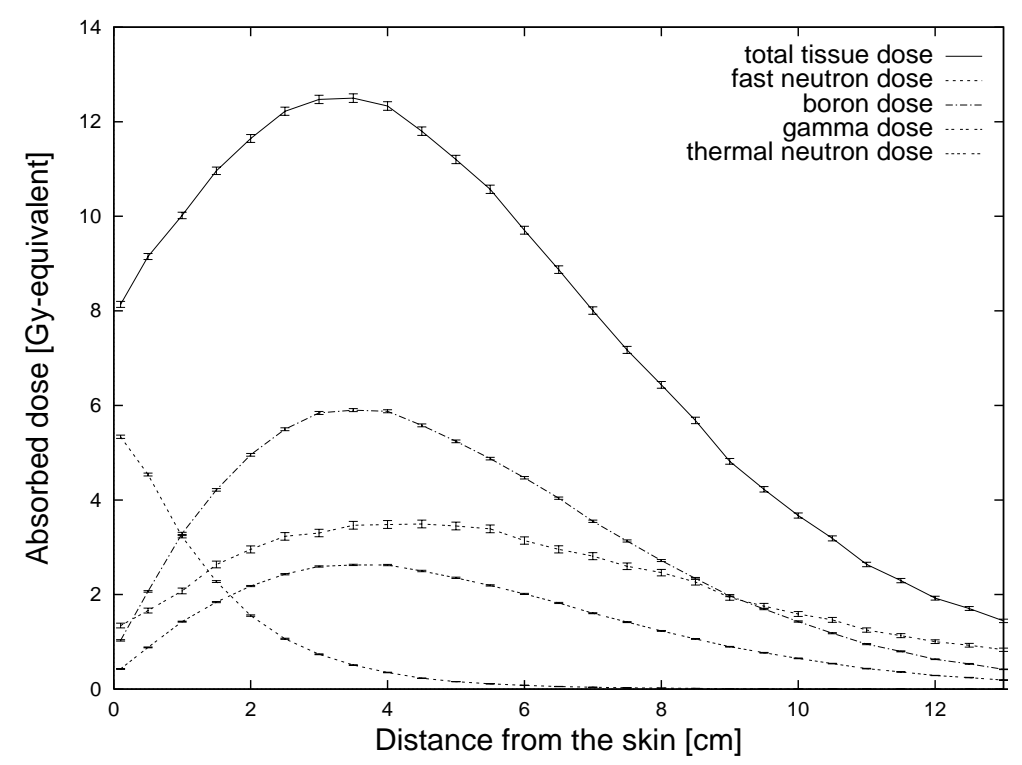

Figure 3.5: Total absorbed healthy-tissue doses (with their components) corresponding to a 12-cm-diam, 7.94-keV monodirectional neutron beam.

For tumors shallower than $6 \mathrm{~cm}$, the therapeutic gains at 2 and $4 \mathrm{~cm}$ are high and vary negligibly with the neutron beam energy between $1 \mathrm{eV}$ and $10 \mathrm{keV}$. They reach their maxima of 5.66 and 5.44 at energies of $3.16 \mathrm{eV}$ and $0.8 \mathrm{keV}$, respectively. Any neutron beam with energy higher than $1 \mathrm{eV}$ and lower than $7.76 \mathrm{keV}$ will result in a relatively high therapeutic gain and in a skin dose lower than 8 Gy-equivalent. However, it should be noted that even if the main tumor mass is shallower than $4 \mathrm{~cm}$, microscopic fingerlets spreading throughout the surrounding tissues can reach greater depths. Since these fingerlets also have to be irradiated, $7.76-\mathrm{keV}$ neutron beams should be preferred for treatment. An overall higher therapeutic gain is obtained with such neutron beams.

Using the therapeutic gain at $8 \mathrm{~cm}$ of 2.52 , the theoretical maximum achievable tumor dose at $8 \mathrm{~cm}$ is 31.5 Gy-equivalent with this 12 - $\mathrm{cm}$-diam, $7.76-\mathrm{keV}$ neutron beam. Without the constraint on the skin dose, 31.9 Gy-equivalent could be achieved with a 12.6$\mathrm{keV}$ neutron beam. Neutrons in the energy range of 2.29 to $16.9 \mathrm{keV}$ will give more than $90 \%$ of the maximum achievable absorbed tumor dose of 31.9 Gy-equivalent. This energy range widens to lower energies as the tumor depth decreases from 8 to $2 \mathrm{~cm}$.

In the case of the absorbed tissue dose per unit neutron emitted by the source, Fig. 3.2 shows that $\mathrm{MD}_{t i}$ increases rapidly for energies above $20 \mathrm{keV}$. For instance, one $14-\mathrm{MeV}$ neutron contributes as much to $\mathrm{MD}_{t i}$ as forty $10-\mathrm{keV}$ neutrons. This is another 
reason why the neutron energy range above $\sim 20 \mathrm{keV}$ is therapeutically undesirable. On the other hand, lower energy neutrons are not nearly as detrimental as fast neutrons. Thermal neutrons contribute only twice as much to the maximum healthy-tissue dose as $10-\mathrm{keV}$ neutrons. With an ideal $7.76-\mathrm{keV}$ neutron beam, each neutron will deliver $44.8 \times 10^{-15}$ Gy-equivalent to the healthy tissues at the location where the absorbed healthy-tissue dose is maximum. The number of neutrons required to reach the 12.5 -Gy-equivalent dose limit in the healthy tissues is $2.79 \times 10^{14}$. Dividing by the area of the beam, this corresponds to a fluence of $2.47 \times 10^{12} \mathrm{n} / \mathrm{cm}^{2}$, or a flux of $6.86 \times 10^{8} \mathrm{n} / \mathrm{cm}^{2} / \mathrm{s}$ for a 1 -h treatment time.

Figure 3.5 shows the total absorbed healthy-tissue dose (with its components) corresponding approximately to the energy of the ideal 12-cm-diam neutron beam. No photons are irradiating the patient in this simulation study. Thus, the gamma dose is strictly induced by interactions of the neutrons with the skin, skull, and brain in the patient head. This induced gamma dose (mainly due to the interactions of neutrons with hydrogen) represents about $16 \%$ of the total tissue dose at the skin.

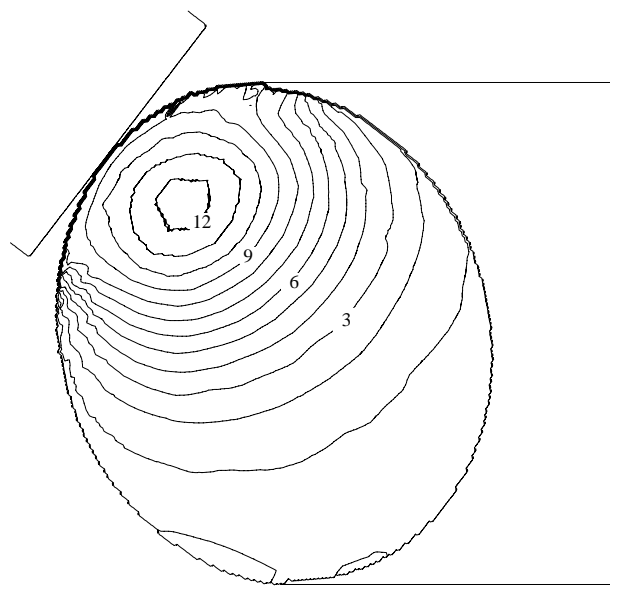

(a)

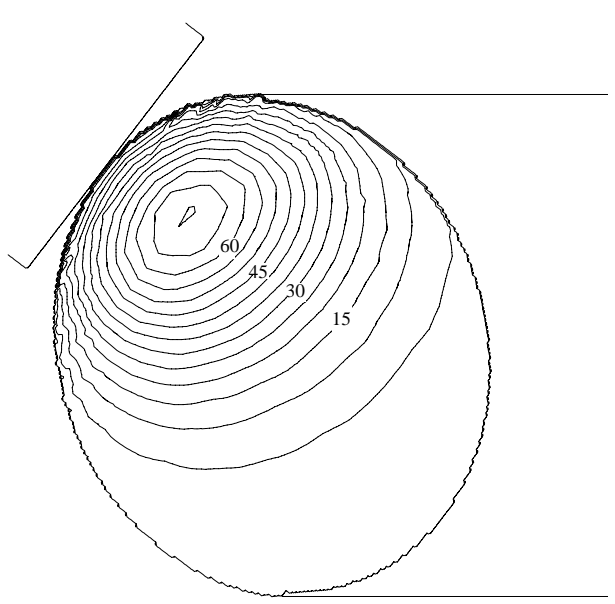

(b)

Figure 3.6: Isodose countour plots of the absorbed (a) healthy-tissue and (b) tumor dose distributions for a 12 -cm-diam neutron beam of energy $7.76 \mathrm{keV}$.

Figures 3.6a and 3.6b show isodose countour plots of the absorbed healthy-tissue and tumor dose distributions corresponding to the ideal 12-cm-diam neutron beam of energy $7.76 \mathrm{keV}$, in the cross-sectional plane of Fig. 3.1b. 


\subsection{Treatment characteristics as a function of the neutron beam diameter}

The analysis of treatment characteristics has been extended to include different neutron beam diameters. The results are shown in Figs. 3.7 and 3.8 for 6 -cm-diam neutron beams, and in Figs. 3.9 and 3.10 for 18 -cm-diam neutron beams. Table 3.1 provides a summary of the results.

The neutron fluence required to reach the 12.5-Gy-equivalent healthy-tissue dose limit decreases with increase in the diameter (see Table 3.1). Numerous simulations of BSAs (see Sec. 4.3) have shown that on a per source neutron basis, the neutron fluence is basically uniform across the exit window of the moderator and independent of the window diameter (for the diameters of interest from 6 through $18 \mathrm{~cm}$ ), as long as the moderator diameter and length are at least two to three times as large as the exit window diameter. Therefore, the decrease in neutron fluence requirement for large-beam diameters shown in Table 3.1 will result in shorter treatment times for an identical neutron yield at the target.

The optimal neutron energy for each tumor depth and each neutron beam diameter is determined using Eqs. 3.5 and 3.6, i.e., by maximizing the ratio $\mathrm{D}_{t u}(x \mathrm{~cm}) / \mathrm{MD}_{t i}$ under the constraint that the skin dose must not exceed 8 Gy-equivalent. For deep-seated tumors at $8 \mathrm{~cm}$, the maximum achievable tumor dose is more than double when the neutron beam diameter increases from 6 to $18 \mathrm{~cm}$. The optimal neutron energy also increases, but only slightly. The increase in tumor dose with beam diameter diminishes for shallower tumors and vanishes for 2-cm-deep tumors. 


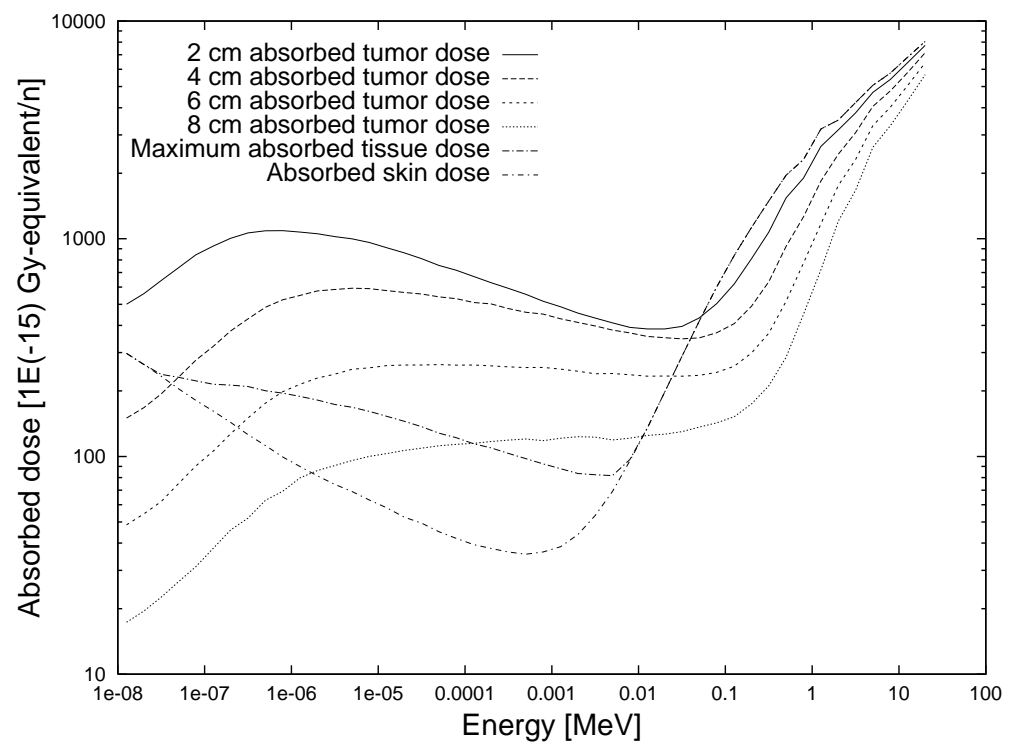

Figure 3.7: Absorbed doses per neutron emitted by the source for a 6-cm-diam monodirectional neutron beam.

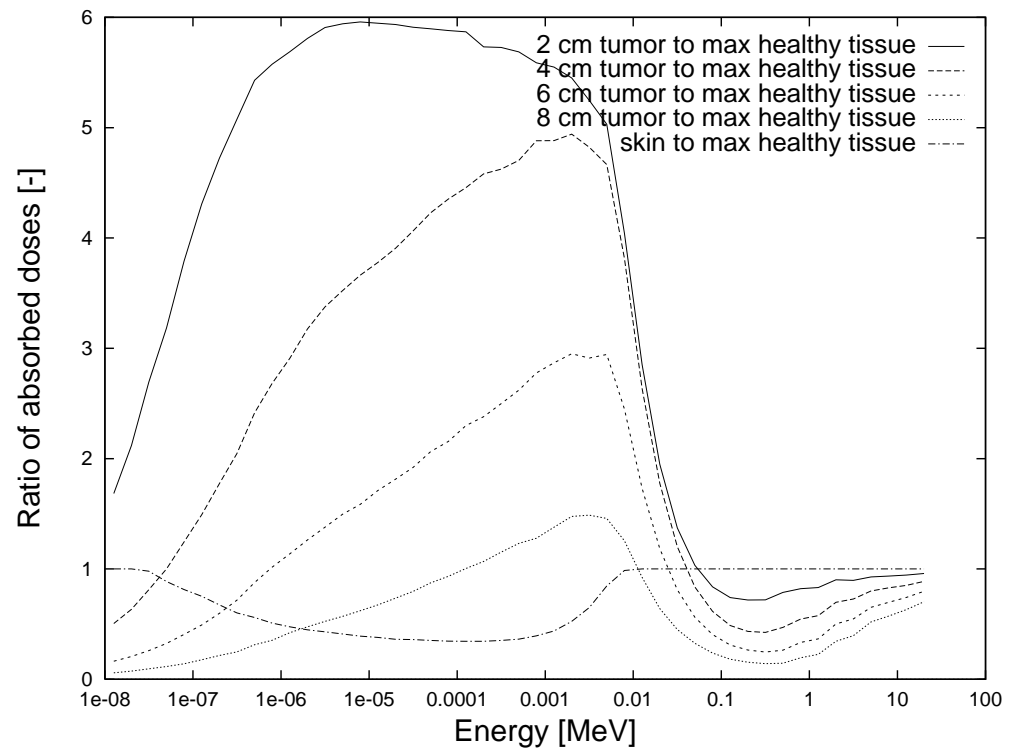

Figure 3.8: Ratios of absorbed doses versus neutron energy for a 6 - $\mathrm{cm}$-diam monodirectional neutron beam. 


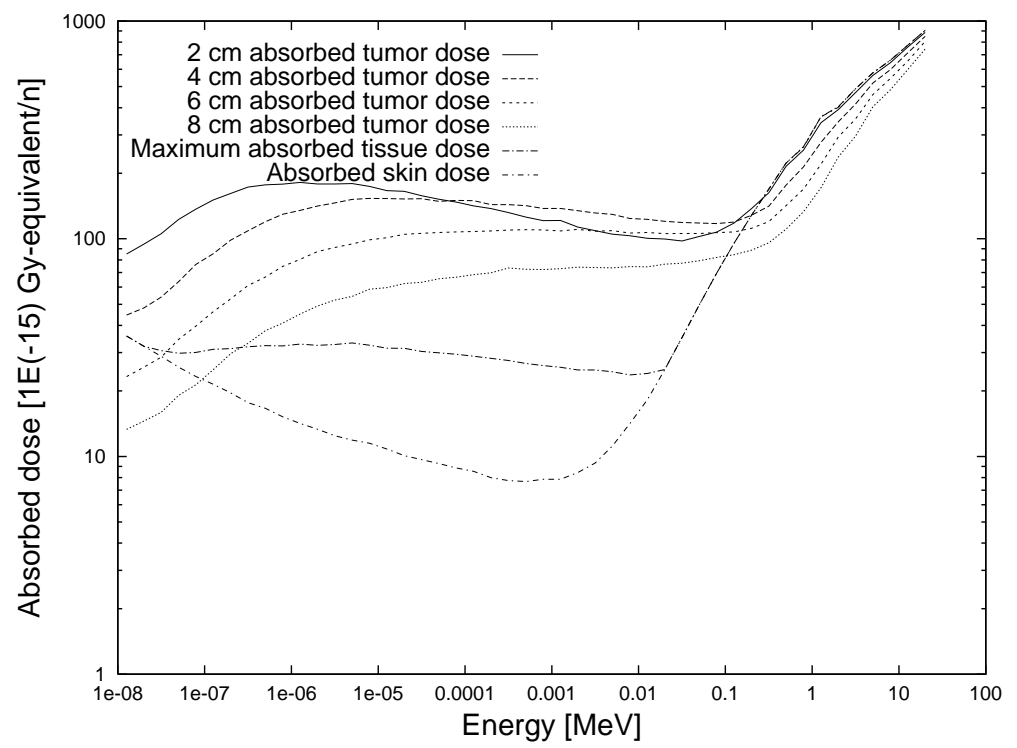

Figure 3.9: Absorbed doses per neutron emitted by the source for a 18-cm-diam monodirectional neutron beam.

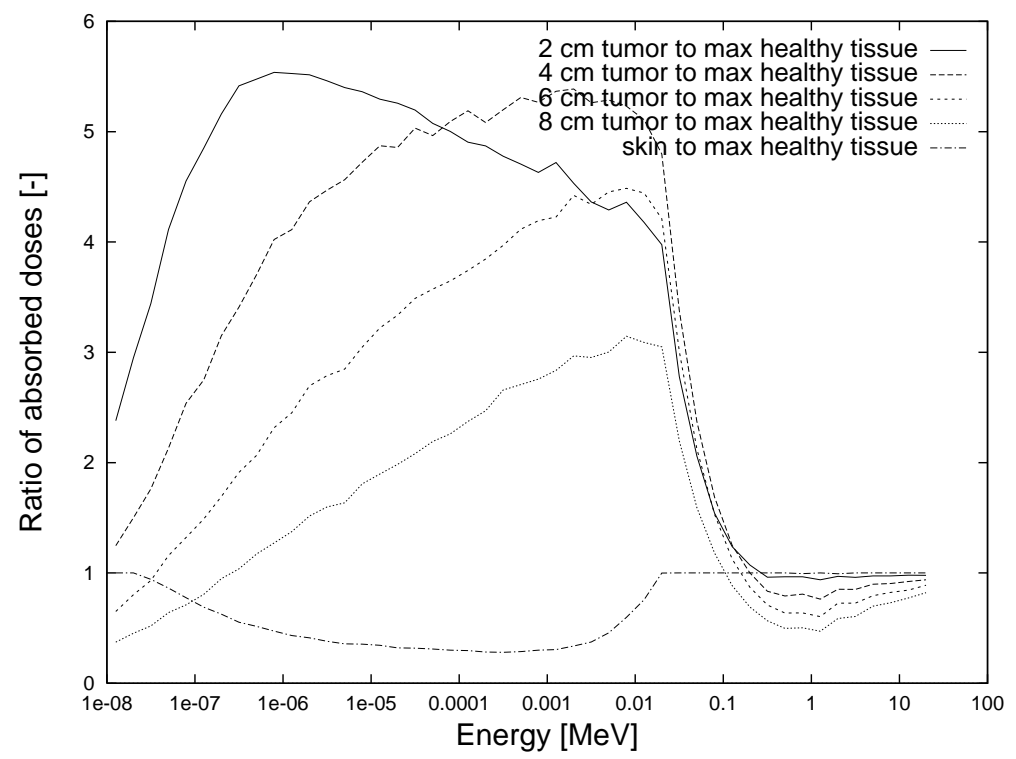

Figure 3.10: Ratios of absorbed doses versus neutron energy for a 18-cm-diam monodirectional neutron beam. 


\begin{tabular}{|c|c|c|c|}
\hline Beam diameter & $6 \mathrm{~cm}$ & $12 \mathrm{~cm}$ & $18 \mathrm{~cm}$ \\
\hline $\begin{array}{l}\text { Neutron fluence required at the entrance } \\
\text { of the skull to reach the } 12.5 \text {-Gy-equivalent } \\
\text { dose limit on the healthy tissues }\left[\mathrm{n} / \mathrm{cm}^{2}\right]\end{array}$ & $5.34 \cdot 10^{12}$ & $2.47 \cdot 10^{12}$ & $2.07 \cdot 10^{12}$ \\
\hline $\begin{array}{l}\text { Neutron flux required for a } \\
1 \text {-h treatment }\left[\mathrm{n} / \mathrm{cm}^{2} / \mathrm{s}\right]\end{array}$ & $14.8 \cdot 10^{8}$ & $6.86 \cdot 10^{8}$ & $5.76 \cdot 10^{8}$ \\
\hline \multicolumn{4}{|l|}{ 8-cm-deep tumor } \\
\hline Optimal energy [keV] & 2.71 & 7.76 & 7.94 \\
\hline $\begin{array}{l}\text { Maximum achievable tumor } \\
\text { dose [Gy-equivalent] }\end{array}$ & 18.5 & 31.5 & 39.3 \\
\hline $\begin{array}{l}\text { Energy range where } 90 \% \text { of the } \\
\text { maximum tumor dose is achieved [keV] }\end{array}$ & 1.08 to 6.67 & 2.29 to 16.9 & 1.23 to 22.6 \\
\hline \multicolumn{4}{|l|}{ 6-cm-deep tumor } \\
\hline Optimal energy [keV] & 2.00 & 7.76 & 7.94 \\
\hline $\begin{array}{l}\text { Maximum achievable tumor } \\
\text { dose [Gy-equivalent] }\end{array}$ & 36.9 & 50.9 & 56.1 \\
\hline $\begin{array}{l}\text { Energy range where } 90 \% \text { of the } \\
\text { maximum tumor dose is achieved }[\mathrm{keV}]\end{array}$ & 0.57 to 6.65 & 0.31 to 16.2 & 0.40 to 21.4 \\
\hline \multicolumn{4}{|l|}{ 4-cm-deep tumor } \\
\hline Optimal energy [keV] & 2.00 & 0.79 & 2.00 \\
\hline $\begin{array}{l}\text { Maximum achievable tumor } \\
\text { dose [Gy-equivalent] }\end{array}$ & 61.8 & 68.0 & 67.3 \\
\hline $\begin{array}{l}\text { Energy range where } 90 \% \text { of the } \\
\text { maximum tumor dose is achieved [keV] }\end{array}$ & 0.13 to 5.71 & 0.03 to 14.3 & 0.01 to 18.8 \\
\hline \multicolumn{4}{|l|}{ 2-cm-deep tumor } \\
\hline Optimal energy [keV] & $7.94 \cdot 10^{-3}$ & $3.16 \cdot 10^{-3}$ & $0.79 \cdot 10^{-3}$ \\
\hline $\begin{array}{l}\text { Maximum achievable tumor } \\
\text { dose [Gy-equivalent] }\end{array}$ & 74.5 & 70.8 & 69.2 \\
\hline $\begin{array}{l}\text { Energy range where } 90 \% \text { of the } \\
\text { maximum tumor dose is achieved [keV] }\end{array}$ & $\begin{array}{l}0.47 \cdot 10^{-3} \\
\text { to } 2.50\end{array}$ & $\begin{array}{l}0.17 \cdot 10^{-3} \\
\text { to } 0.41\end{array}$ & $\begin{array}{l}0.16 \cdot 10^{-3} \\
\text { to } 0.09\end{array}$ \\
\hline
\end{tabular}

Table 3.1: Dosimetric properties of monodirectional neutron beams as a function of their diameter.

From these results, one could conclude that larger beams have better treatment characteristics than narrow ones. Indeed, reduction in treatment times and enhancements in tumor doses are advantages of large diameter beams. However, they are also accompanied by disadvantages highlighted in Figs. 3.11 and 3.12, which show isodose countour plots of the absorbed healthy-tissue and tumor dose distributions for the optimal 6 - and 18 -cmdiam neutron beams, respectively. The healthy-tissue dose distribution corresponding to the 6 -cm-diam neutron beam is characterized by a well-localized, but fast decreasing peak 
dose. The volume of healthy tissues receiving large doses (e.g., 6 Gy-equivalent) is smaller than for the case of the 18-cm-diam neutron beam. The advantage of this narrow beam is

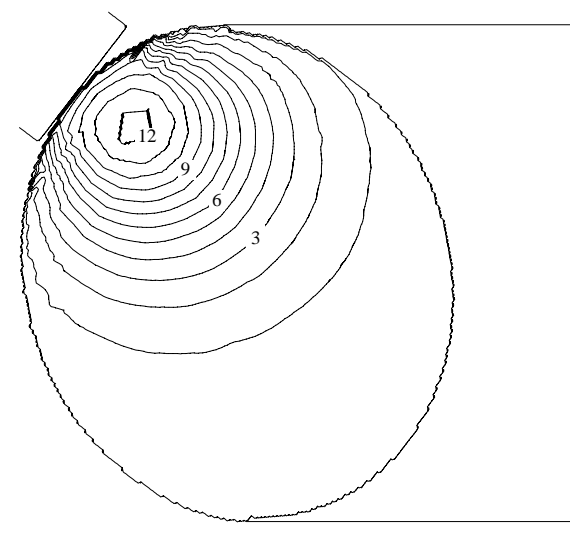

(a)

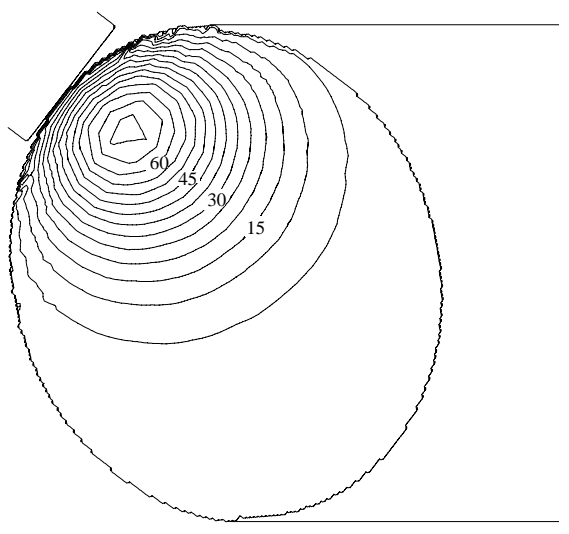

(b)

Figure 3.11: Isodose countour plots of the absorbed (a) healthy-tissue and (b) tumor dose distributions for a 6 -cm-diam neutron beam of energy $2.71 \mathrm{keV}$.

now obvious. It allows one to reduce the volume of healthy tissues exposed to high doses. Even though the healthy-tissue doses are much lower than the tumor doses, it is always desirable to limit radiation exposure to healthy tissues as much as possible. There are several cases when narrow beams would be better suited than broader beams. For a smalldiameter tumor mass located at a distance of less than $4 \mathrm{~cm}$ from the skin and which has

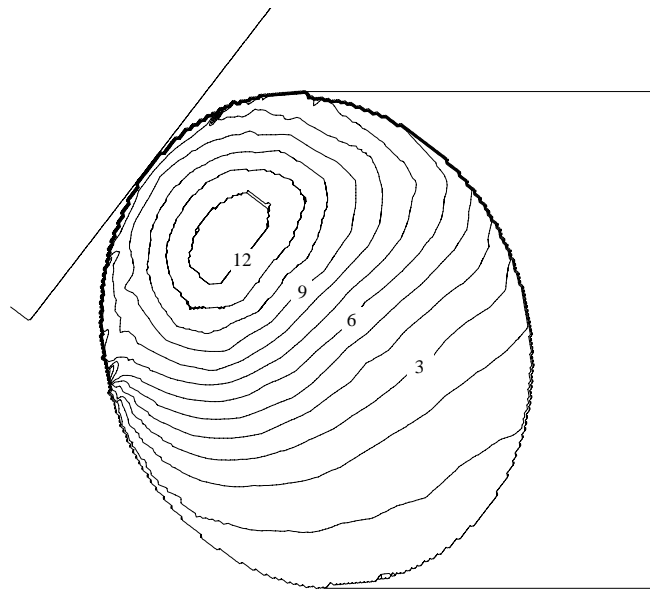

(a)

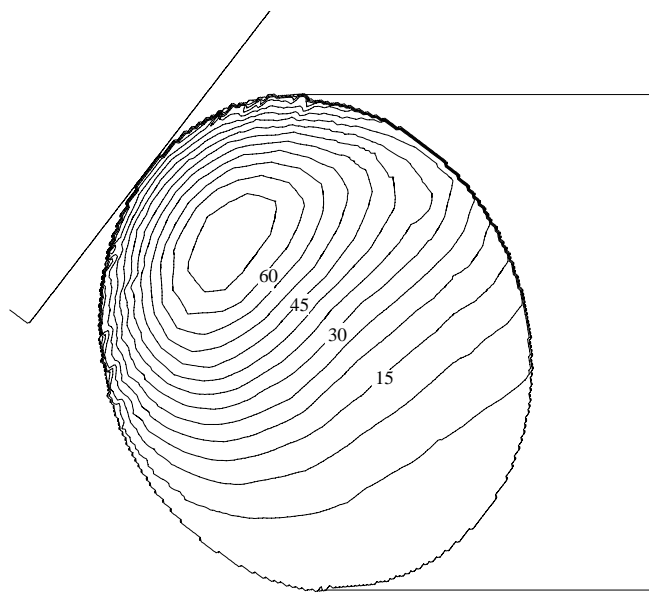

(b)

Figure 3.12: Isodose countour plots of the absorbed (a) healthy-tissue and (b) tumor dose distributions for an 18-cm-diam neutron beam of energy $7.94 \mathrm{keV}$. 
not spread deeply in the brain, the narrow beam would be perfectly suitable. It would give a maximum absorbed tumor dose of 70.3 Gy-equivalent at $3.0 \mathrm{~cm}$ from the skin and more than 60 Gy-equivalent to all the points between the depths 1.4 and $4.2 \mathrm{~cm}$. The distance at which the healthy-tissue dose decreases to 6.25 Gy-equivalent (i.e., half its maximum value) is $6.4 \mathrm{~cm}$. The 12 - and $18-\mathrm{cm}$-diam neutron beams are more penetrating but do not result in higher absorbed tumor doses. These beams lead to larger volumes of high tumor doses, but also to larger volumes of high healthy-tissue doses. The half healthy-tissue dose distances are much larger, 7.5 and $9.0 \mathrm{~cm}$, respectively. These larger volumes of high tumor doses are not necessary if the tumor is small. In this case, one can significantly reduce the radiation exposure to healthy tissues by using a narrow beam. Cases where narrow beams would be advantageous are shallow melanoma tumors, and deep-seated tumors that have been removed macroscopically by surgical procedure, but for which microscopic fingerlets still require open-skull radiation treatment, etc.

This simulation analysis applies only to the case of single-neutron beams. The results do not apply to the case of multiple-neutron beams, in which case the healthytissue dose contributions of the different neutron beams are added to satisfy the total dose limit of 12.5 Gy-equivalent at one point in the brain.

\subsection{Summary of results and discussion}

A monoenergetic neutron beam simulation study has been carried out to determine which neutron energy is the most suitable for treatment of shallow and deep-seated brain tumors in the context of BNCT. Table 3.1 summarizes the results. For deep-seated tumors, the energy range $1 \mathrm{keV}$ to $20 \mathrm{keV}$ appears to be optimal, with a maximum therapeutic gain at $\sim 8 \mathrm{keV}$. For shallow tumors, any neutron beam of energy higher than $1 \mathrm{eV}$ and lower than $\sim 8 \mathrm{keV}$ will result in a relatively high therapeutic gain. However, it should be noted that even if the main tumor mass is shallow, microscopic fingerlets spreading throughout the surrounding tissues can reach greater depths. Since these fingerlets also have to be irradiated, 8 -keV neutron beams should be preferred for treatment of shallow tumors. An overall higher therapeutic gain is obtained with such neutron beams.

The neutron beam diameter has a considerable effect on the tumor dose for deepseated tumors. For instance, the tumor dose at a depth of $8 \mathrm{~cm}$ becomes more than double when the neutron beam diameter increases from 6 to $18 \mathrm{~cm}$. The drawback of larger 
diameter beams is that the volume of high healthy-tissue dose also increases with the beam diameter. For shallow tumors, the increase in tumor dose with beam diameter is still present but less pronounced, and is not significant for 2-cm-deep tumors. However, the volume of high healthy-tissue dose still increases with the beam diameter. From the results of this neutron beam energy and diameter simulation study, one concludes that 8-keV neutron beams are optimal for deep-seated tumors, but no set of neutron beam diameter and energy is best for any kind of tumor. The diameter and energy of the neutron beam have to be determined by the characteristics of the tumor to be treated, such as tumor depth, size, and how far from the main tumor mass the fingerlets have spread. Given all these characteristics, simulation of particle transport in the brain can help one to determine the optimal set of neutron beam diameter and energy for treatment.

This analysis is only valid for the boron concentrations, relative biological effectivenesses, compound factors and dose limit on the healthy tissues given in Sec. 3.2. With the development of new pharmaceuticals, one can expect a considerable improvement of the tumor-seeking compounds. These would deliver more boron atoms to the target area, the cancerous cells. It is arguable whether this enhancement of the boron concentration in the tumor cells would be beneficial for the therapy. One has to consider the ratio of the boron concentrations in the tumor and in the healthy-tissue cells to answer this question. If this ratio is equal to the present one of 3.5, the boron uptake enhancement will result in dose distributions similar to the present ones. If the ratio is higher than 3.5 , the boron uptake enhancement will result in higher tumor doses for the same healthy-tissue doses, and the maximum achievable tumor doses shown in Table 3.1 will increase. This increase in tumor doses will however not be proportional to the ratio of the boron concentrations, because of the contributions of the neutron and photon doses to the total dose in Eq. 3.8. Enhancement of the tumor compound factor due to improvement of pharmaceuticals would also have a beneficial effect on the tumor doses, unless it is accompanied by a proportional increase in the normal tissue compound factor. If either the boron concentration in the tumor cells or the tumor compound factor is increased, the treatment time will be shorter, because it would take a lower neutron fluence to reach the dose limit of 12.5 Gy-equivalent on the healthy tissues.

Even though the dose limit on the healthy tissues is currently set to 12.5 Gyequivalent by BNL's protocol [17], this value might increase in the future, as more clinical trials are performed. Whatever this dose limit might become, the ratios shown in Figs. 3.3, 
3.8 and 3.10 will remain the same. The tumor and skin doses could then be obtained by multiplying the ratios in these figures by the new healthy-tissue dose limit. If the healthytissue dose limit increases by $20 \%$ to 15 Gy-equivalent, the tumor dose curves will also be $20 \%$ higher. It is important to observe that the ratio $\mathrm{D}_{s k} / \mathrm{MD}_{t i}$ will now have to be lower than $8 / 15=0.53$, instead of $8 / 12.5=0.64$. In the case of a 12 -cm-diam neutron beam, the optimal energy $E_{\text {optimal }}$ will therefore decrease slightly (see Fig. 3.3) and the therapeutic ratios for deep-seated tumors will be slightly lower. However, the maximum achievable tumor doses will overall still increase. In the limiting case where the maximum healthytissue dose would be as high as 29 Gy-equivalent, the ratio $\mathrm{D}_{s k} / \mathrm{MD}_{t i}$ would have to be lower than 0.28 . This occurs only for neutron beam energies of $\sim 0.2 \mathrm{keV}$, as shown in Fig. 3.3. In this particular case, the 8-cm-deep tumor dose would be as high as 57 Gy-equivalent. For higher healthy-tissue dose limits, the 8 Gy-equivalent skin dose limit can no longer be satisfied. 57 Gy-equivalent appears thus to be the maximum achievable 8-cm-tumor dose with the existing skin dose limit, RBE factors, compound factors, and boron concentrations. Since the neutron fluence and the healthy-tissue dose limit are proportional, the treatment time will increase proportionally with the healthy-tissue dose limit. 


\section{Chapter 4}

\section{Design of BSAs for BNCT for the D-D and D-T neutron sources}

\subsection{Introduction}

In the previous Chapter, we concluded that neutron beams for BNCT should have a specific energy distribution in order to maximize the tumor dose and to minimize the healthy tissue dose. Another constraint for this radiation therapy is the treatment time. The boron-bearing tumor-seeking compound bound to the tumor cells diffuses away after a few hours, and the treatment becomes then less efficient. Moreover, for the comfort of the patient, a 5 -h treatment is undesirable. Although fractionated treatment schemes could be adopted, this option still limits the total treatment time to a few hours or tens of hours. The neutron flux thus has to be sufficiently high.

Initially, only reactors were thought to be capable of delivering the necessary fluence of neutrons in suitable lengths of time. The problem with reactors is that they are very expensive and too large to be used in hospitals.

As an alternative to nuclear reactors, accelerator-based neutron sources are being considered for future use in hospitals. Such sources offer the potential for improved patient treatments in addition to avoid the above problems associated with reactor installations. The most appealing of the alternatives that have been suggested is the use of proton accelerators with low-Z targets. Several neutronics studies have been published on the design of accelerator-based neutron sources $[22,23,24,25]$. The maximum neutron energy from an 
accelerator-based neutron source utilizing the ${ }^{7} \operatorname{Li}(p, n)$ reaction is significantly below fission neutron energies, thus requiring less moderation. This can be exploited to achieve clinically superior depth-dose distributions [26]. For example, an accelerator-based BNCT facility is under development at the Lawrence Berkeley National Laboratory [27]. The ${ }^{7} \operatorname{Li}(p, n)$ reaction at proton energies of about $2.5 \mathrm{MeV}$ will be utilized since it offers a high neutron yield in combination with a low maximum neutron energy. Treatment times of 40 min were estimated for this accelerator [27] to treat a patient in a single-treatment session. In addition, the quality of the treatment is higher than for the existing reactor-based treatment facilities if one evaluates it on the figures-of-merit given previously. This source is probably one of the best achievable for BNCT. However, this accelerator-based treatment facility is still very expensive and large, even though it is not as large as a reactor-based one. Also, the target design could be difficult, due to the low melting point $\left(179^{\circ} \mathrm{C}\right)$ of metallic lithium.

The focus of this Chapter is on the fusion reactions ${ }^{2} \mathrm{H}(d, n)^{3} \mathrm{He}$ (deuteriumdeuterium) and ${ }^{3} \mathrm{H}(d, n)^{4} \mathrm{He}$ (deuterium-tritium), which have - to the best of author's knowledge - not yet been investigated in detail as potential neutron sources for BNCT. The monoenergetic neutrons emitted by these reactions have energies of about 2.43 and 14.1 MeV, respectively, for an incident deuteron beam with energy ranging between 100 and $400 \mathrm{keV}$.

The use of these two fusion reactions could result in an accelerator and target system simpler and less expensive than the current ones, while satisfying all of the requirements for BNCT. While the protons for the ${ }^{7} \operatorname{Li}(p, n)$ or ${ }^{9} \mathrm{Be}(p, n)$ reactions need to be accelerated between 2.5 and $4.0 \mathrm{MeV}$ for the reactions to occur with a sufficient neutron yield, the deuteron beam energy required for D-D and D-T reactions lies between 100 and $400 \mathrm{keV}$. Because of this lower beam energy, smaller accelerators with higher currents could be utilized.

In this Chapter, we present a study to determine which types of moderator and reflector are the most suitable to reduce the neutron energies to therapeutically useful regions, without suffering large losses in neutron beam intensity. The Monte-Carlo codes MCNP [20] and BNCT Radiation Treatment Planning Environment (BNCT_RTPE) software [28] are used for the neutron transport calculation. A comparison of two different methods for the dose distribution computation in the brain is also presented. 


\subsection{Neutron source characterization}

Neutrons from the D-D and D-T fusion reactions have to be moderated down to the desired epithermal or thermal energy range by means of a beam-shaping assembly (BSA). The following sections discuss the characteristics of the neutron source and the BSA.

In our numerical simulations, the neutron source is characterized as follows. Neutrons are emitted isotropically and monoenergetically across a 5-cm-diam flat circular surface. The source is distributed uniformly over the surface of the target, which is composed of a 10- $\mu$ m-thick titanium layer on a 1-mm-thick copper substrate, water-cooled on the back by 3 -mm-deep water channels machined in a 5-mm-thick stainless steel plate. The spread in the D-D and D-T neutron energies due to deuteron straggling in the target is not considered in this study. The assumption of isotropy has to be discussed in detail. Concerning the D-T reaction, the high $Q$-value for the reaction makes the neutron energy relatively insensitive to the angle of emission for the region of low deuteron energy $(\sim 100 \mathrm{keV})$ [8]. The neutron energy varies around $14.1 \mathrm{MeV}$ by only $\pm 7 \%$ over all solid angles for a deuteron beam energy of $200 \mathrm{keV}$. The neutrons are emitted practically isotropically in the center-of-mass system below this energy. In the lab system, the differential cross section for the D- $\mathrm{T}$ reaction varies by only $\pm 7 \%$ for the same $200-\mathrm{keV}$ deuteron beam. Thus, angular isotropy in the lab system is an adequate approximation for deuteron beams of low energy. For D-D, the angular distribution in the center-of-mass system is anisotropic. A better modeling of the source accounting for the angular distribution would be required, but this is beyond the scope of these calculations.

\subsection{Beam-shaping assembly description}

Source neutrons enter a cylindrical BSA with the monoenergetic neutron distribution corresponding to D-D or D-T reactions. They travel through the BSA, which contains several layers of different materials until they reach the other side where the phantom head is located (see Fig. 4.1). A 13.4-cm-thick lithiated polyethylene delimiter [10, 29], separating the BSA from the phantom head, restricts the neutron flux to a 12-cm-diam window. Its

role is to decrease radiation exposure to the organs other than the brain. The angle of the exit window of the delimiter was directly taken from Ref. [10]. No analysis of the possible influence of this angle on the treatment characteristics has been performed in this study. 


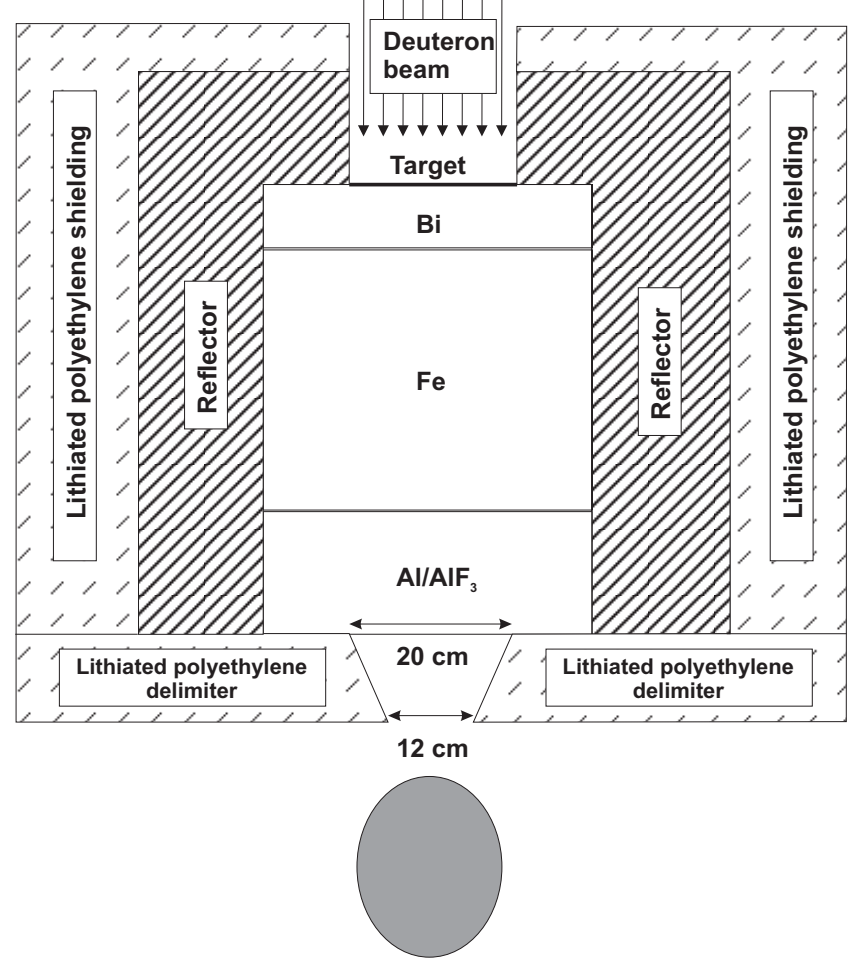

Figure 4.1: BSA showing the 20-cm-diam exit window where the neutron and photon spectra are calculated, referred to as exit window throughout the text.

The neutron beam diameter has been set equal to values used by other groups $[10,30]$ in order to compare the dose distributions in the brain.

\subsection{Estimation of absorbed doses in the brain}

Two different methods are used to compute the dose distribution in the patient's head. In the first method (method A), the neutron and photon transport simulation from the neutron source to the phantom head is performed entirely by the MCNP code [20]. Different variance reduction techniques, such as geometry splitting with Russian roulette and angle biasing with DXTRAN are used to decrease the simulation time. The second method (method B) is based on the coupling of the Monte-Carlo codes MCNP [20] and BNCT_RTPE [28]. MCNP is used to simulate the neutron and photon transport through the BSA. The energy and angular- dependent neutron and photon distributions are calculated at the exit of the BSA across the 20-cm-diam exit window (see Fig. 4.1). This window 
is referred to as the exit window throughout the text for simplicity. These distributions are then used by the BNCT_RTPE code for the radiation transport through the lithiated polyethylene delimiter and the phantom head. For both methods, the boron concentrations and RBE values used for the dose computations are taken from BNL's protocol [17] and are listed in Sec. 3.2. Since a boron concentration of $10 \mu \mathrm{g} / \mathrm{g}$ is used in method A for the neutron transport simulation in the soft tissues, the same concentration is used in method $\mathrm{B}$. The special-purpose BNCT_RTPE software system developed at the Idaho National Engineering and Environmental Laboratory is a tested, fully developed treatment- planning program, currently approved for use in a dosimetry protocol. This program has been used for the ongoing BNCT clinical trials at the Brookhaven Medical Research Reactor (BMRR).

The advantages of method A over method B are: (a) method A tracks the neutrons and photons transmitted through the delimiter and (b) it accounts for the neutrons and photons reflected from the head and the delimiter back to the BSA. However, method A has the disadvantage of longer computational time.

In addition to the advantages mentioned above, the main reason for computing the dose distributions with method A rather than with method B - using the well-established treatment planning code BNCT_RTPE — is the following. The delimiter [29] used in our numerical simulations has not been designed for high-energy neutron sources, which can generate high-energy photons by interactions with the moderator. Since the D-D and D$\mathrm{T}$ reactions emit high-energy neutrons, it is necessary to determine whether the delimiter still fulfills its role, i.e., still reduces radiation exposure to the organs other than the brain. Because method $\mathrm{B}$ does not track the neutrons and photons transmitted through the delimiter to the phantom, method A must be used to assess the performance of the delimiter. Method A is first compared with method B in Sec. 4.4.3. before being used extensively in the following sections.

\subsubsection{Tissue composition}

The densities and elemental compositions of the skin, bone, and brain used by BNCT_RTPE are slightly different from the ones used in the MIRD-5 [21] phantom head. To compare the doses computed by BNCT_RTPE with the ones computed with our model, we will use the densities and elemental compositions of BNCT_RTPE. They are taken directly from Ref. [31] and listed in Table 4.1. 


\begin{tabular}{|c||c|c|c|}
\hline Element [w/o] & Skin & Bone & Brain \\
\hline \hline $\mathrm{H}$ & 10.39 & 4.99 & 10.56 \\
\hline $\mathrm{C}$ & 23.74 & 21.14 & 13.95 \\
\hline $\mathrm{N}$ & 2.69 & 3.99 & 1.84 \\
\hline $\mathrm{O}$ & 62.98 & 43.38 & 72.59 \\
\hline $\mathrm{Na}$ & - & 0.10 & 0.14 \\
\hline $\mathrm{P}$ & - & 8.08 & 0.39 \\
\hline $\mathrm{Cl}$ & 0.21 & 0.28 & 0.14 \\
\hline $\mathrm{K}$ & - & - & 0.39 \\
\hline $\mathrm{Ca}$ & - & 17.55 & - \\
\hline $\mathrm{Mg}$ & - & 0.20 & - \\
\hline $\mathrm{S}$ & - & 0.30 & - \\
\hline \hline$\rho\left[\mathrm{g} / \mathrm{cm}^{3}\right]$ & 1.07 & 1.61 & 1.047 \\
\hline
\end{tabular}

Table 4.1: Elemental compositions in weight percent fractions and densities in grams per cubic centimetre of the skin, bone, and brain used in BNCT_RTPE.

\subsubsection{Fluence-to-KERMA conversion factors}

In BNCT_RTPE, the doses associated with nitrogen absorption and proton recoil reactions are computed separately using two different fluence-to-KERMA conversion factors. Goorley et al. [31] showed that the sum of these two fluence-to-KERMA conversion factors was sensibly equal to the fluence-to-KERMA conversion factor for all relevant reactions in brain tissues. To be consistent with the change in elemental compositions in the brain, the fluence-to-KERMA conversion factors for the neutrons have to be modified. The fluenceto-KERMA conversion factors for the neutrons computed with the elemental compositions listed in Table 4.1 are shown in Fig. 2.4 and listed in appendix B.4. They were taken from Ref. [15]. They differ from the ones used in Sec. 3.2 (ICRU report 46 [14]) only at low neutron energies. The photon mass attenuation coefficients (ICRU report 46 [14]) and the ${ }^{10} \mathrm{~B}$ fluence-to-KERMA conversion factors (listed in Caswell et al. [16]) are not different.

\subsubsection{Comparison of method A with method B}

In this section, the dose distributions and treatment times obtained by method A and method $\mathrm{B}$ are compared on a sample problem. D-T neutrons are being considered. The BSA used for comparison has a diameter of $30 \mathrm{~cm}$. It is composed of $5 \mathrm{~cm}$ of Bi, $50 \mathrm{~cm}$ of Fe, $24 \mathrm{~cm}$ of $40 \% \mathrm{Al} / 60 \% \mathrm{AlF}_{3}, 1 \mathrm{~mm}$ of ${ }^{6} \mathrm{LiF}$, and $1 \mathrm{~mm}$ of $\mathrm{Pb}$; and is surrounded by a $30-$ cm-thick $\mathrm{Al}_{2} \mathrm{O}_{3}$ reflector. For the dose computations in the phantom head, BCNT_RTPE 
uses cubic volumes of $1 \mathrm{~cm}^{3}$. Therefore, the cylinder - in which the absorbed doses are computed by method A (see Fig. 3.1) - has a diameter set equal to $1 \mathrm{~cm}$.

Since neutrons and photons transmitted through the delimiter separating the BSA from the phantom head (see Fig. 4.1) are not tracked to the head in method B, we will first compute the dose distribution obtained with method A without tracking the neutrons and photons around the exit window. Absorbed healthy-tissue and tumor doses are compared in Figs. 4.2 and 4.3. A good agreement is observed between the two methods. We observe

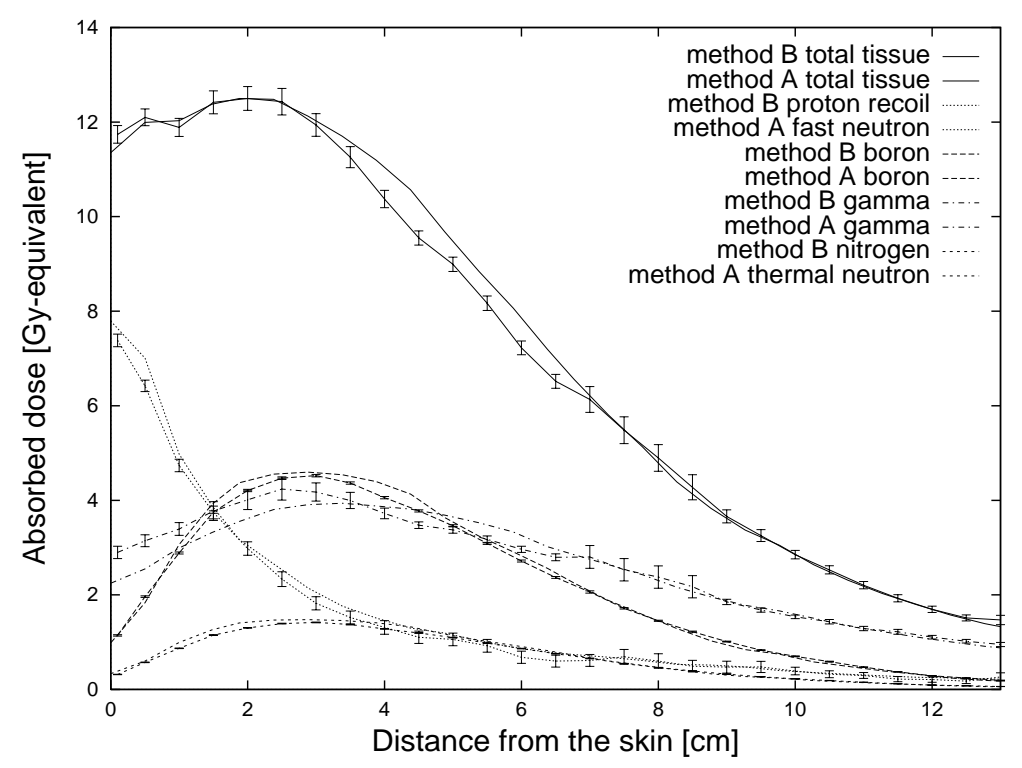

Figure 4.2: Comparison of absorbed healthy-tissue doses computed with method A (shown with errorbars) and method B. Neutrons and photons were not tracked through the delimiter.

that the thermal- and fast neutron absorbed doses are very close to the nitrogen absorption and proton recoil absorbed doses, respectively. This demonstrates that the thermaland fast neutron doses are mostly due to nitrogen absorption and proton recoil reactions, respectively. A perfect agreement between the two methods is not expected since the head geometries used in the two methods are slightly different.

By tracking the neutrons and photons transmitted through the delimiter, one can obtain the dose distribution shown in Fig. 4.4, which is compared with the case without tracking. We observe that all absorbed dose components are higher than when the particles transmitted through the delimiter were not tracked, which is not surprising. The advantage of method A over method B is clear because it accounts for the presence of particles 


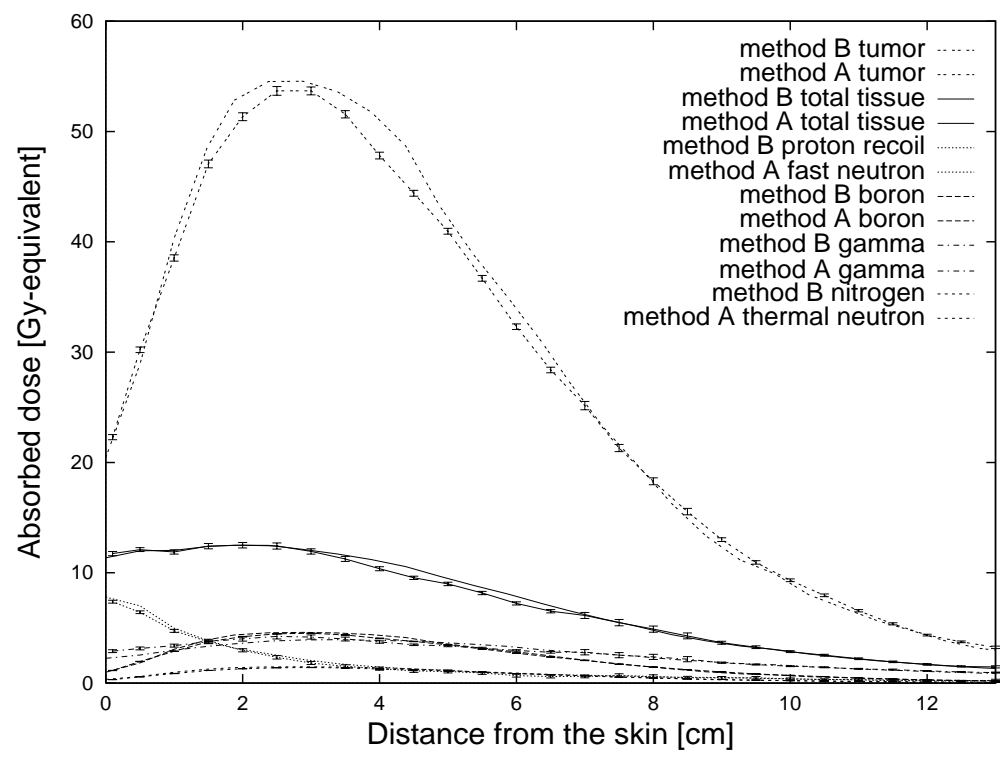

Figure 4.3: Comparison of absorbed tumor doses computed with method A (shown with errorbars) and method B. Neutrons and photons were not tracked through the delimiter.

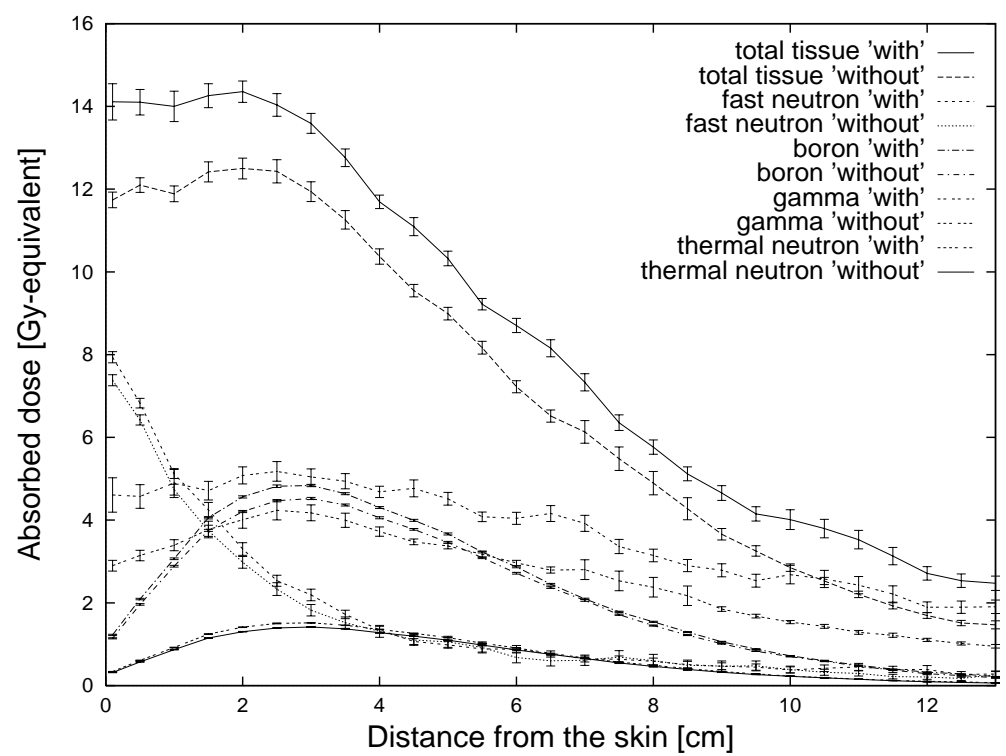

Figure 4.4: Comparison of absorbed healthy-tissue doses computed with method A with and without the particles transmitted through the delimiter. The dose distributions are normalized to reach a maximum absorbed healthy-tissue dose of 12.5 Gy-equivalent for the distribution neglecting the leakage through the delimiter.

transmitted through the delimiter and contributing to the doses in the phantom head. This contribution is simply omitted in method B. While neglecting this contribution may be valid 
for low-energy neutron sources, it is no longer valid for high-energy ones, which give rise to high-energy photons. In our case, for instance, the contribution of the photons transmitted through the delimiter is about $30 \%$ of the total photon dose.

Method A highlights a deficiency in the role of the delimiter, which was originally designed to absorb neutrons specifically. A layer of lead could be added to reduce the undesired photon dose. More discussion on the effect of this photon dose is included in Sec. 4.8.1. Method A is used for the dose computations in the following sections.

\subsection{Methodology for the optimization of the BSA}

Neutrons from both D-D and D-T fusion reactions have to be moderated down to the desired epithermal energy range. This section focuses on how different combinations of materials are being determined to shape the most suitable neutron beam. An entrance dose limit to the skin of 10 Gy-equivalent was used in the optimization process in order to compare our treatment characteristics with the ones of other groups [10] using $10 \mathrm{~Gy}-$ equivalent for the skin dose limit.

First, several materials were analyzed separately to determine their effect on the neutron spectrum. Then, different combinations of materials were considered, with the goal to produce an intense, broad energy epithermal beam peaking around $8 \mathrm{keV}$, with the fast and thermal neutron components reduced to a minimum level. Neutron spectra are analyzed at the exit of the BSA across the 20-cm-diam exit window shown in Fig. 4.1. Unless specified otherwise, the BSA used for the optimization was $25 \mathrm{~cm}$ in diameter and surrounded by a thin $(0.5-\mathrm{mm})$ layer of ${ }^{6} \mathrm{LiF}$ and a $30-\mathrm{cm}$-thick $\mathrm{Al}_{2} \mathrm{O}_{3}$ reflector, as recommended in Ref. [10].

The first step of the optimization was based on a "trial-and-error" process. One would try one set of materials and observe the neutron spectrum obtained, as well as its intensity. Then, the thickness of one material was varied, or a new material was substituted for another, and one would obtain a new neutron spectrum. Comparing the two neutron spectra, one would decide which one is better, in terms of their intensity, and their thermal, epithermal and fast neutron flux components.

Once a reasonably good neutron spectrum is obtained, the BSA is further improved using the quantities defined in Sec. 3.2 of Chapter 3 : the absorbed tumor dose $\mathrm{D}_{t u}(\mathrm{x})$ at a depth of $\mathrm{x} \mathrm{cm}$, the skin dose $\mathrm{D}_{s k}$ and the maximum healthy-tissue dose $\mathrm{MD}_{t i}$ per neutron emitted by the source, as well as the treatment time $\mathrm{T}$. Knowing the number $\mathrm{N}$ of source 
neutrons required to satisfy the dose limit on the healthy tissues, i.e., $\mathrm{N} \cdot \mathrm{MD}_{t i}=12.5 \mathrm{~Gy}-$ equivalent, the skin and tumor doses are given by $\mathrm{N} \cdot \mathrm{D}_{s k}$ and $\mathrm{N} \cdot \mathrm{D}_{t u}$, respectively. Several parameters can be varied for the optimization, the materials $M_{i}$, the number I of different materials, the material thicknesses $\mathrm{Th}_{i}$, the BSA diameter $\mathrm{D}$, the reflector material $\mathrm{M}_{\text {refl }}$, the reflector thickness $\mathrm{Th}_{\text {refl }}$, etc. Even though one could allow these parameters to vary to infinity, one will set limits on their ranges. Because of weight concerns, the BSA diameter D will vary in the range 25 to $80 \mathrm{~cm}$, the reflector thickness $T h_{r e f l}$ in the range 0 to $20 \mathrm{~cm}$. The number of materials I in the BSA will be limited to 3 . The reflector materials $\mathrm{M}_{\text {refl }}$, as well as the neutron moderating materials $\mathrm{M}_{i}$ will be chosen among those commonly used for BNCT. Let $\vec{y}$ be an array containing all the variable parameters.

$$
\vec{y}=\left(M_{i}(1 \leq i \leq I) \quad T h_{i}(1 \leq i \leq I) \quad D \quad M_{\text {refl }} \quad T h_{\text {refl }}\right)
$$

The optimal BSA will be such that the set of parameters gives the highest tumor dose at $\mathrm{x}$ $\mathrm{cm}$

$$
D_{t u}\left(x, \vec{y}_{\text {optimal }}\right)=\max \left(D_{t u}(x, \vec{y})\right),
$$

for all possible $\vec{y}$, under the following constraints:

$$
T\left(\vec{y}_{\text {optimal }}\right) \leq T_{\max }
$$

for the treatment time $\mathrm{T}$,

$$
N \cdot D_{\text {sk }}\left(\vec{y}_{\text {optimal }}\right) \leq 10 \text { Gy-equivalent }
$$

for the skin dose, and

$$
N \cdot M D_{t i}\left(\vec{y}_{o p t i m a l}\right)=12.5 \quad \text { Gy-equivalent }
$$

for the maximum healthy-tissue dose. The number of source neutrons $\mathrm{N}$ is determined using Eq. 4.5. The treatment time $\mathrm{T}$ is determined using Eq. 3.7. The neutron source strength $\mathrm{S}$ depends on the fusion reaction considered. In this Chapter, we will use $1.5 \times 10^{12} \mathrm{n} / \mathrm{s}$ for D-D (see Sec. 4.7) and $10^{14} \mathrm{n} / \mathrm{s}$ for D-T (see Sec. 4.8.3). The latter will be decreased to $7.5 \times 10^{13} \mathrm{n} / \mathrm{s}$ for the final treatment time calculations, for reasons explained in Sec. 4.8.3. The maximum permissible treatment time $\mathrm{T}_{\max }$ is set to $45 \mathrm{~min}$.

To illustrate graphically the optimization procedure, the absorbed dose at a depth of $8 \mathrm{~cm} \mathrm{D}_{t u}(8 \mathrm{~cm})$, the skin dose $\mathrm{D}_{s k}$, and the treatment time $\mathrm{T}$ are plotted as a function of 
the variable parameter $\mathrm{y}_{i}$, for four different cases in Figs. 4.5 through 4.8. The horizontal dotted line indicates (a) the upper limit of 10 Gy-equivalent on the absorbed skin dose $\mathrm{D}_{s k}$ (see left ordinate scale) and (b) the upper limit of $\mathrm{T}_{\max }$ on the treatment time $\mathrm{T}$ (see right ordinate scale). This dotted line is horizontal because these limits depend on (a) biological criteria and (b) clinical constraints rather than on the variable parameter $y_{i}$.

In Fig. 4.5, the skin dose $\mathrm{D}_{s k}$ and the treatment time $\mathrm{T}$ are lower than $10 \mathrm{~Gy}$ equivalent and $\mathrm{T}_{\max }$, respectively, for the range of $\mathrm{y}_{i}$ values indicated by the continuous line ended on both sides by arrows. In this range, $\mathrm{D}_{s k}$ and $\mathrm{T}$ are within the problem constraints imposed by Eqs. 4.4 and 4.3. The absorbed tumor dose $\mathrm{D}_{t u}(8 \mathrm{~cm})$ is maximum for $y_{i}=70$



Figure 4.5: Optimization for a case where the absorbed skin dose and treatment time constraints are not limiting the maximum achievable tumor dose $\mathrm{D}_{t u}(8 \mathrm{~cm})$. The optimal value of $\mathrm{y}_{i}$ is $70 \mathrm{~cm}$.

$\mathrm{cm}$. This optimal value of $\mathrm{y}_{i}$ is indicated by an " $\mathrm{x}$ " in the graph.

Figure 4.6 illustrates a case similar to the one shown in Fig. 4.5, but where the skin dose curve is different. It is lower than 10 Gy-equivalent for all values of $y_{i}$ smaller than $60 \mathrm{~cm}$, and greater otherwise. The maximum value of $\mathrm{D}_{t u}(8 \mathrm{~cm})$ is still obtained for a $y_{i}=70 \mathrm{~cm}$, but the skin dose constraint of Eq. 4.4 imposes values of $\mathrm{y}_{i}$ lower than $60 \mathrm{~cm}$. Therefore, $\mathrm{D}_{t u}(8 \mathrm{~cm})$ is maximum under the constraint of Eq. 4.4 for $y_{i}=60 \mathrm{~cm}$.

For the two first cases considered, the time constraint imposed by Eq. 4.3 was not limiting the maximum $\mathrm{D}_{t u}(8 \mathrm{~cm})$ achievable. Figure 4.7 illustrates a case similar to the one shown in Fig. 4.5, but the treatment time is now limiting the maximum tumor dose 


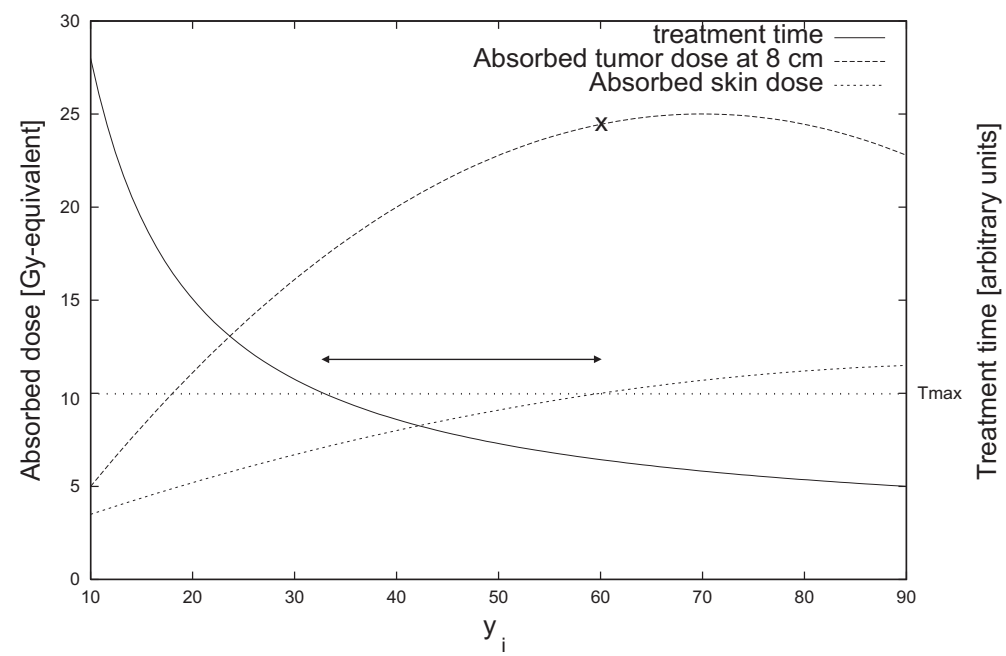

Figure 4.6: Optimization for a case similar to the one shown in Fig. 4.5, but where the skin dose curve is different. The maximum tumor dose $\mathrm{D}_{t u}(8 \mathrm{~cm})$ achievable is limited by the skin dose constraint. The optimal value of $y_{i}$ is $60 \mathrm{~cm}$.

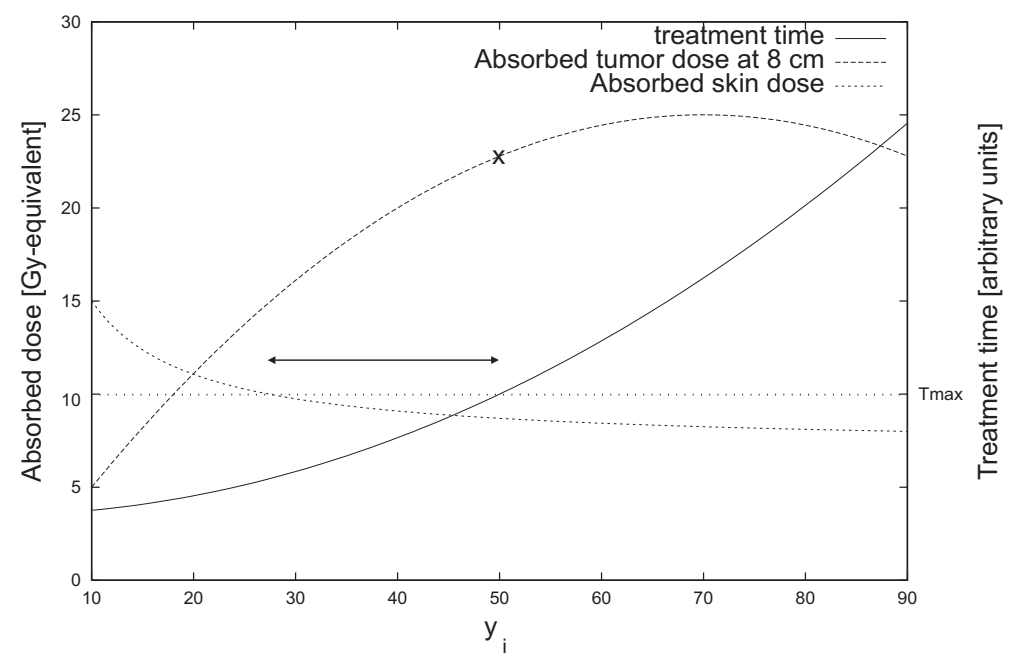

Figure 4.7: Optimization for a case similar to the one shown in Fig. 4.5, but where the treatment time constraint limits the maximum achievable tumor dose $\mathrm{D}_{t u}(8 \mathrm{~cm})$. The optimal value of $\mathrm{y}_{i}$ in this case is $50 \mathrm{~cm}$.

achievable $\mathrm{D}_{t u}(8 \mathrm{~cm})$. The treatment time is lower than $\mathrm{T}_{\text {max }}$ for all values of $\mathrm{y}_{i}$ smaller than $50 \mathrm{~cm}$, and greater otherwise. Even though the maximum tumor dose $\mathrm{D}_{t u}(8 \mathrm{~cm})$ is achieved for $y_{i}=70 \mathrm{~cm}$, the treatment time constraint of Eq. 4.3 imposes $y_{i}=50 \mathrm{~cm}$.

In Fig. 4.8, neither the treatment time constraint, nor the skin dose constraint are satisfied. However, the skin dose $\mathrm{D}_{s k}$ is approximately constant over the range of $\mathrm{y}_{i}$ values, 


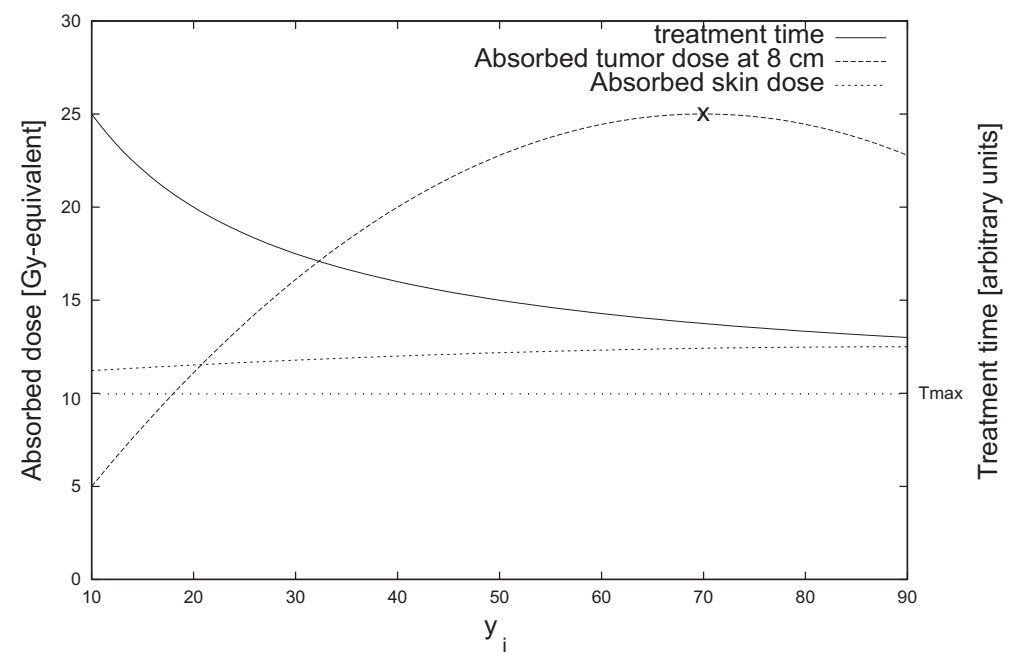

Figure 4.8: Optimization for a case where none of the two constraints can be satisfied. The optimal $\mathrm{y}_{i}$ in this case is chosen such that the tumor dose $\mathrm{D}_{t u}(8 \mathrm{~cm})$ is maximized.

and the treatment time $\mathrm{T}$ decreases quickly to approach $\mathrm{T}_{\max }$ asymptotically for large $\mathrm{y}_{i}$ values. Therefore, $\mathrm{y}_{i}$ should preferrably be chosen as large as possible. Since coincidentally the tumor dose $\mathrm{D}_{t u}(8 \mathrm{~cm})$ curve reaches its maximum for large $\mathrm{y}_{i}$ values, $\mathrm{y}_{i}$ can be chosen such that it maximizes $\mathrm{D}_{t u}(8 \mathrm{~cm})$. The optimal $\mathrm{y}_{i}$ in this case is $70 \mathrm{~cm}$. One should however eventually try to be within the constraints imposed by Eqs. 4.4 and 4.3 . This can be done by changing the values of the other variable parameters in the array $\vec{y}$.

This work of optimization should be performed for all the independent variables of the BSA. With 3 materials in the moderator assembly, the number of independent variables is 9 . The ratio $D_{t u}(x, \vec{y})$ is a 9-dimensional hypersurface imbedded in a 10-dimensional hyperspace. Because of the complexity of this optimization problem, one has to make some assumptions. Let $\vec{y}_{s u b}$ be the set of parameters $\vec{y}$ minus its $\mathrm{M}_{r e f l}$ and $\mathrm{Th}_{\text {refl }}$ components.

$$
\vec{y}_{s u b}=\left(M_{i}(1 \leq i \leq 3) \quad T h_{i}(1 \leq i \leq 3) \quad D\right) .
$$

One will assume that if the reflector material $\mathrm{M}_{\text {refl }}$ and thickness $\mathrm{Th}_{\text {refl }}$ are optimal for one subset $\vec{y}_{s u b}$ of parameters, they will be optimal for all subsets $\vec{y}_{s u b}$ of parameters. The same assumption will be made for the moderator diameter $\mathrm{D}$. With these assumptions, one can optimize (a) the reflector material and thickness, and (b) the moderator diameter, without optimizing simultaneously the other parameters. Once the ratio $D_{t u}(x, \vec{y})$ is maximized in terms of these parameters, the optimization problem is reduced to finding the maximum of a 6-dimensional hypersurface in a 7-dimensional hyperspace. The optimization must be per- 
formed with the constraints aforementioned. To simplify the optimization, we will consider only the moderating materials frequently used in the literature on BNCT: lead, bismuth, iron, lithium fluoride $\mathrm{LiF}$ enriched in either ${ }^{6} \mathrm{Li}$ or ${ }^{7} \mathrm{Li}$, the mixture $40 \% \mathrm{Al} / 60 \% \mathrm{AlF}_{3}$, magnesium fluoride $\mathrm{MgF}_{2}$ and heavy water. The only reflector materials considered were lead, aluminum oxide $\mathrm{Al}_{2} \mathrm{O}_{3}$ and graphite.

Knowing the absorbed tumor dose $\mathrm{D}_{t u}(\mathrm{x})$, the skin dose $\mathrm{D}_{s k}$ and the treatment time $\mathrm{T}$ for different sets of parameters $\vec{y}$, one can choose the one that is optimal in terms of Eqs. 4.2 through 4.5. Once a reasonably good set $\vec{y}$ has been found, one can vary the material thicknesses by small values to observe the influence of these parameters on the doses and treatment time and to converge to an even better set $\vec{y}$. Optimization processes based on that scheme are known as "climbing" procedures and are completely described in the literature [32]. The method used here is similar to the one described on pages 271 through 284 of Ref. [32]. This method leads to the optimal set of parameters that satisfies the constraints of the problem.

Since we did not have the priviledge to do an infinite number of simulations, the optimization did not intend to find the set of absolutely optimal parameters. Instead, we found a set of parameters that was optimal among those tried, and for which slight variations in material thicknesses did not improve the results, but rather worsened them.

\subsubsection{Method based on the multigroup neutron diffusion equations}

An analytical method based on the time-independent multigroup neutron diffusion equations could also be used for the optimization process. In these equations, the neutron energy range must be broken down into $\mathrm{G}$ energy groups, 1 being the fast neutron group, while $\mathrm{G}$ is the thermal energy group. The neutron energy range of the $\mathrm{g}^{\text {th }}$ group is $E_{g}$ to $E_{g-1} . \mathrm{S}_{g}$ is the rate at which neutrons appear in group $\mathrm{g}$ in units $\mathrm{n} / \mathrm{cm}^{3} / \mathrm{s}$. In the particular cases of the D-D and D-T neutron sources, all neutrons are born in the first energy group. A scattering collision can change the neutron energy and hence either remove it from the group $g$, or if it is initially in another group g', scatter it to an energy in the group g. We will characterize the probability for scattering a neutron from a group $g$ ' to the group $g$ by something akin to the differential scattering cross section $\Sigma_{s}\left(E_{g^{\prime}} \rightarrow E_{g}\right), \Sigma_{s g^{\prime} g}$. We will assume that neutrons always lose energy by scattering.

The material constants and neutron fluxes used in the multigroup diffusion equa- 
tions are now defined explicitly. The neutron flux $\phi_{g}(x)$ in the $\mathrm{g}^{\text {th }}$ energy group is defined as

$$
\phi_{g}(x)=\int_{E_{g}}^{E_{g-1}} \phi(E, x) d E .
$$

The total cross section characterizing the group $\mathrm{g}$ is defined as

$$
\Sigma_{t g}(x)=\frac{\int_{E_{g}}^{E_{g-1}} \Sigma_{t}(E, x) \phi(E, x) d E}{\phi_{g}(x)} .
$$

For the scattering terms, the group-transfer cross section is

$$
\Sigma_{s g^{\prime} g}(x)=\frac{\int_{E_{g}}^{E_{g-1}}\left(\int_{E_{g^{\prime}}}^{E_{g^{\prime}-1}} \Sigma_{s}\left(E^{\prime} \rightarrow E, x\right) \phi\left(E^{\prime}, x\right) d E^{\prime}\right) d E}{\phi_{g^{\prime}}(x)} .
$$

The multigroup neutron diffusion equations are approximations of the neutron transport equations. The approximations are based on Fick's law, which introduces a relationship between the neutron current $\mathrm{J}_{g}(\mathrm{x})$ and the neutron flux $\phi_{g}(\mathrm{x})$ :

$$
J_{g}(x)=-D_{g}(x) \nabla \phi_{g}(x)
$$

The diffusion coefficient $\mathrm{D}_{g}(\mathrm{x})$ is such that the leakage from group $\mathrm{g}$ can be written within the diffusion approximation as $\nabla D_{g} \nabla \phi_{g}[33]$. The diffusion coefficient $\mathrm{D}_{g}(\mathrm{x})$ is computed using

$$
D_{g}(x)=\frac{\int_{E_{g}}^{E_{g-1}} D(E, x) \nabla \phi(E, x) d E}{\int_{E_{g}}^{E_{g-1}} D(E, x) \nabla \phi(E, x) d E} .
$$

Most of the quantities above require the knowledge of the neutron flux $\phi(x)$, which is just the function that we are trying to calculate in the first place by discretizing the energydependent diffusion equation in energy. Hence it seems as if our development is somewhat circular. In reality, approximations of the neutron flux $\phi(x)$ are used to compute the material constants $\Sigma_{t g}(\mathrm{x}), \mathrm{D}_{g}(\mathrm{x})$ and $\Sigma_{s g^{\prime} g}(\mathrm{x})$. We will not consider these approximations in detail, since they are available in the literature [33].

Having defined the material constants and the neutron fluxes for the different energy groups, we can now derive the time-independent multigroup neutron diffusion equations:

$$
-\nabla D_{g} \nabla \phi_{g}+\Sigma_{R g} \phi_{g}=\sum_{g^{\prime}=1}^{g^{\prime}=g-1} \Sigma_{s g^{\prime} g} \phi_{g^{\prime}}+s_{g} \quad 1 \leq g \leq G .
$$

In these equations, $\Sigma_{R g}$ is the removal cross section of the neutron energy group $g$ defined as

$$
\Sigma_{R g}=\Sigma_{t g}-\Sigma_{s g g}
$$


Hence, we have a set of G coupled diffusion equations for the G unknown group fluxes $\phi_{g}(\mathrm{x})$.

To approximate the BSA, the time-independent diffusion equations are written in a one-dimensional slab geometry. The BSA is composed of several layers of materials with different thicknesses. Neutrons are produced in the first layer (target) in the first energy group, they travel through several materials before they reach a tissue layer which represents the patient's head. We will assume L layers, the first layer is located between the coordinates $\mathrm{x}_{1}$ and $\mathrm{x}_{2}$, the second layer between $\mathrm{x}_{2}$ and $\mathrm{x}_{3}$, etc. The neutron flux in the $1^{\text {th }}$ layer will be denoted as $\phi_{g}^{(l)}(\mathrm{x})$. At the interface between two materials, we have the following boundary conditions:

$$
\begin{aligned}
& \phi_{g}^{(l)}\left(x_{l+1}\right)=\phi_{g}^{(l+1)}\left(x_{l+1}\right) \\
& J_{g}^{(l)}\left(x_{l+1}\right)=J_{g}^{(l+1)}\left(x_{l+1}\right)
\end{aligned}
$$

for $1 \leq g \leq G$ and $1 \leq l \leq L-1$, which are equalities of neutron fluxes and neutron currents at the interfaces between two layers. On the left side of material 1, we apply the following boundary condition:

$$
\phi_{g}^{(1)}\left(x_{1}-2.13 \cdot D_{g}^{(1)}\right)=0 \quad 1 \leq g \leq G,
$$

while on the right side of material L, we have

$$
\phi_{g}^{(L)}\left(x_{L+1}+2.13 \cdot D_{g}^{(L)}\right)=0 \quad 1 \leq g \leq G
$$

These two conditions impose vanishing neutron fluxes at a small distance away from the first and last layers, respectively.

The solutions to Eqs. 4.12 for the first energy group read

$$
\phi_{1}^{(l)}(x)=A_{1}^{(l)} \exp \left(\frac{x}{L_{1}^{(l)}}\right)+C_{1}^{(l)} \exp \left(-\frac{x}{L_{1}^{(l)}}\right)+\frac{s_{1}^{(l)}}{\Sigma_{R, 1}^{(l)}}
$$

for $1 \leq l \leq L . \mathrm{A}_{1}^{(l)}$ and $\mathrm{C}_{1}^{(l)}$ are constants to be determined by the boundary conditions above. In this equation, we made use of the diffusion length L, defined as

$$
L^{2}=\frac{D}{\Sigma_{R}}
$$

The volumetric neutron source strength $s_{1}^{(l)}$ is equal to 0 for 1 different from 1 .

The system of equations for the first energy group can be written in the form

$$
A \cdot \vec{x}=\vec{b} .
$$


For 3 material layers, the matrix $\mathrm{A}$ is equal to

$$
\left(\begin{array}{cccccc}
e^{\frac{x_{1}-2.13 D_{1}^{(1)}}{L_{1}^{(1)}}} & e^{-\frac{x_{1}-2.13 D_{1}^{(1)}}{L_{1}^{(1)}}} & 0 & 0 & 0 & 0 \\
e^{\frac{x_{2}}{L_{1}^{(1)}}} & e^{-\frac{x_{2}}{L_{1}^{(1)}}} & -e^{\frac{x_{2}}{L_{1}^{(2)}}} & -e^{-\frac{x_{2}}{L_{1}^{(2)}}} & 0 & 0 \\
-\frac{D_{1}^{(1)}}{L_{1}^{(1)}} e^{\frac{x_{2}}{L_{1}^{(1)}}} & \frac{D_{1}^{(1)} e^{-\frac{x_{2}}{L_{1}^{(1)}}}}{L_{1}^{(1)}} & \frac{D_{1}^{(2)}}{L_{1}^{(2)}} e^{\frac{x_{2}}{L_{1}^{(2)}}} & -\frac{D_{1}^{(2)}}{L_{1}^{(2)}} e^{-\frac{x_{2}}{L_{1}^{(2)}}} & 0 & 0 \\
0 & 0 & e^{\frac{x_{3}}{L_{1}^{(2)}}} & e^{-\frac{x_{3}}{L_{1}^{(2)}}} & -e^{\frac{x_{3}}{L_{1}^{(3)}}} & -e^{-\frac{x_{3}}{L_{1}^{(3)}}} \\
0 & 0 & -\frac{D_{1}^{(2)}}{L_{1}^{(2)}} e^{\frac{x_{3}}{L_{1}^{(2)}}} & \frac{D_{1}^{(2)}}{L_{1}^{(2)}} e^{-\frac{x_{3}}{L_{1}^{(2)}}} & \frac{D_{1}^{(3)}}{L_{1}^{(3)}} e^{\frac{x_{3}}{L_{1}^{(3)}}} & -\frac{D_{1}^{(3)}}{L_{1}^{(3)}} e^{-\frac{x_{3}}{L_{1}^{(3)}}} \\
0 & 0 & 0 & 0 & e^{\frac{x_{3}+2.13 D_{1}^{(3)}}{L_{1}^{(3)}}} & e^{-\frac{x_{3}+2.13 D_{1}^{(3)}}{L_{1}^{(3)}}}
\end{array}\right)
$$

while the vectors $\vec{x}$ and $\vec{b}$ are equal to

$$
\left(\begin{array}{c}
A_{1}^{(1)} \\
C_{1}^{(1)} \\
A_{1}^{(2)} \\
C_{1}^{(2)} \\
A_{1}^{(3)} \\
C_{1}^{(3)}
\end{array}\right) \text { and }\left(\begin{array}{c}
-\frac{s_{1}^{(1)}}{\Sigma_{R, 1}} \\
0 \\
0 \\
0 \\
0 \\
0
\end{array}\right)
$$

For G energy groups and $\mathrm{L}$ material layers, the system will consist of $2 \cdot L \cdot G$ equations. Such a system can easily be solved using some numerical linear algebra routine for lower triangular systems of equations. We can easily vary the thicknesses of the material layers and obtain the solution for the vector of unknowns $\vec{x}$.

Once the system of equations is solved in terms of the unknowns $\vec{x}$, i.e. $\mathrm{A}_{g}^{(l)}$ and $\mathrm{C}_{g}^{(l)}$, one can compute the different components of the tissue and tumor doses using Eq. 2.11 discretized in energy in the tissue layer L. The thermal neutron dose $\mathrm{D}_{t h}$ for instance reads

$$
D_{t h}(x)=k_{n, G} \phi_{G}^{(L)}(x)
$$

where $\mathrm{k}_{n, G}$ is the fluence-to-KERMA conversion factor for neutrons in tissues averaged over the energy range $\mathrm{E}_{G}$ to $\mathrm{E}_{G-1}$. The averaging over energy is performed using an equation of the same form as Eq. 4.8. The fast neutron dose $\mathrm{D}_{f}$ reads

$$
D_{f}(x)=\sum_{g=1}^{G-1} k_{n, g} \phi_{g}^{(L)}(x),
$$


while the boron dose reads

$$
D_{B}(x)=C_{B} \sum_{g=1}^{G} k_{B, g} \phi_{g}^{(L)}(x),
$$

where $C_{B}$ is the ${ }^{10} B$ concentration in either normal cells or tumor cells. The total tissue and tumor doses are then computed using Eq. 2.15. One should notice that no information is available for the photon doses, because photon fluxes are not computed by the multigroup diffusion equations. This is one disadvantage of this method.

Knowing the unknown vector $\vec{x}$ for different sets of materials and thicknesses, one can find the corresponding tissue doses, tumor doses and treatment time. The optimization procedure is then similar to the one described in Sec. 4.5. One varies the material thicknesses by small values to observe the influence of these parameters on the doses and the treatment time, until the optimal set of parameters that satisfies the constraints of the problem is found.

The advantage of this method based on the time-independent multigroup diffusion equations is that it is much faster than optimizing with Monte-Carlo simulations. However, this method also presents several disadvantages compared to methods based on Monte-Carlo simulations. It is not as accurate because (a) it is a one-dimensional model and therefore it simulates the three-dimensional geometry only approximately, (b) it is a multigroup method, which means that the cross sections are averaged over energy ranges, as opposed to MonteCarlo methods which use cross sections continuous in energy, (c) it uses Fick's law as an approximation to solve the neutron transport equations, while Monte-Carlo simulations model the physics exactly, $(d)$ it does not account for the photon dose in the normal tissue and tumor dose computations.

The method based on the multigroup diffusion equations could be used to obtain a good starting point in the optimization process. Materials and material thicknesses could be estimated with this method. Once a reasonably good starting point is obtained, MonteCarlo simulations are however necessary to obtain more accurate results and to pursue the research of the optimal set of materials and material thicknesses. Even though it presents advantages, the method based on the multigroup diffusion equations will not be used for the optimization problem in this work. Monte-Carlo simulations will be performed for all stages of the optimization. 


\subsection{Selection of filter configurations}

It is worth briefly examining the cross sections of the different materials considered for neutron moderation. The data are taken from the MCNP [20] cross section library. As shown in Figs. 4.9 and 4.10, lead and bismuth have high cross sections for the $(n, 2 n)$

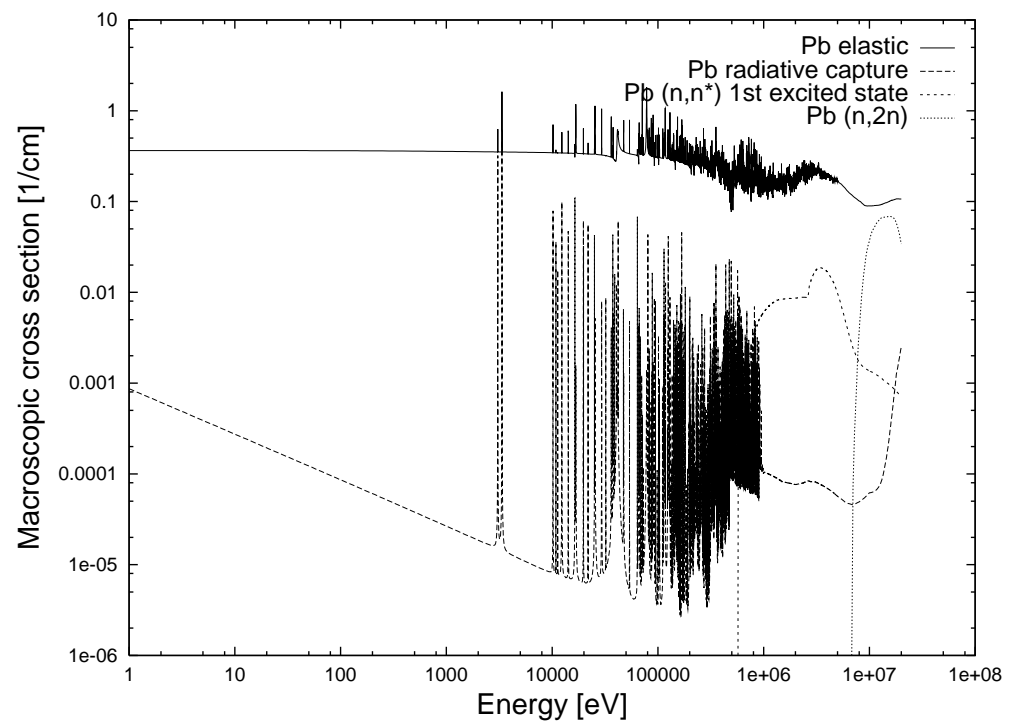

Figure 4.9: $\mathrm{Pb}$ macroscopic cross sections.

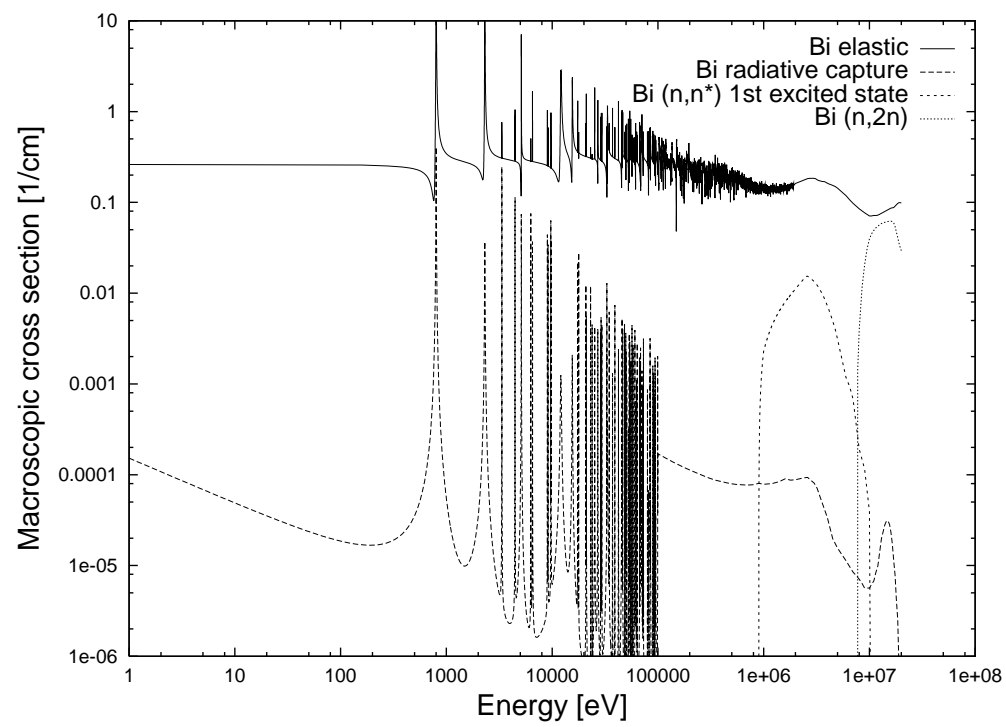

Figure 4.10: Bi macroscopic cross sections. 
reactions at energies above $10 \mathrm{MeV}$. Thus, lead and bismuth could be used to enhance the number of neutrons through $(n, 2 n)$ reactions with $14.1-\mathrm{MeV}$ neutrons in the case of the D-T neutron source. On the other hand, lead is also a good photon absorber. Thin layers of lead will be used at the end of the moderation to decrease the undesired photon dose.

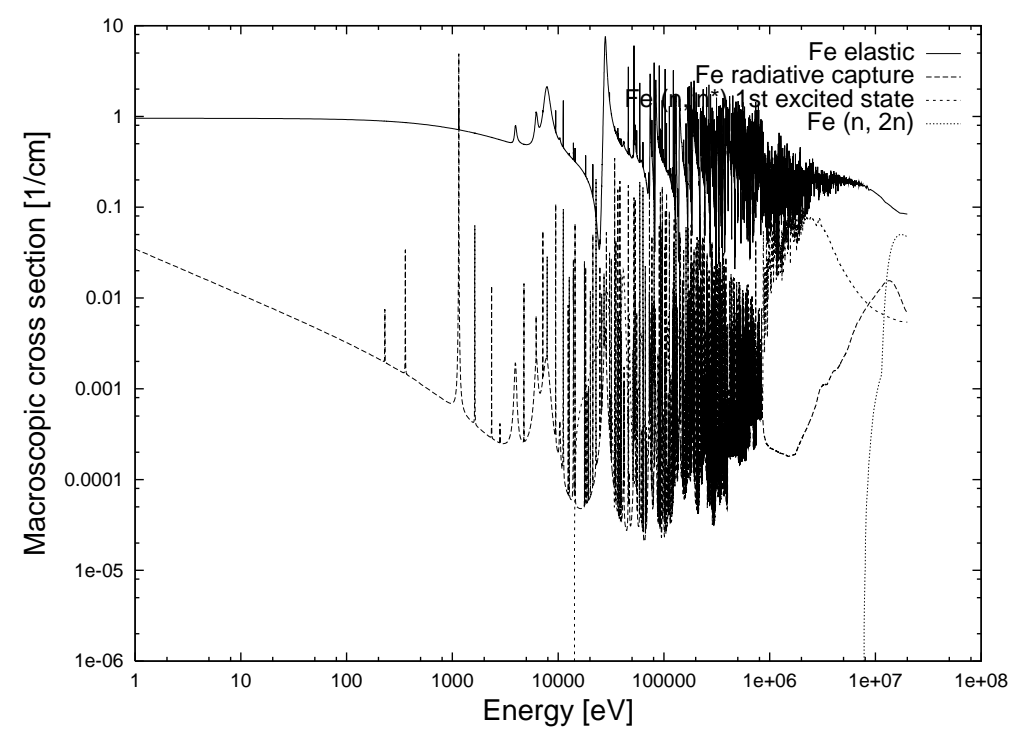

Figure 4.11: Fe macroscopic cross sections.

Iron has a less-pronounced $(n, 2 n)$ reaction and a higher absorption cross section than lead (see Fig. 4.11). However, iron is a good moderator at high energies due to its high inelastic scattering cross section above $860 \mathrm{keV}$. Moreover, it has a window at $20 \mathrm{keV}$ with low cross sections, just around the desired neutron energy. Thus, iron is used to moderate neutrons from both reactions.

Though widely utilized as a neutron moderator, heavy water is of no interest in our case. Because of the presence of deuterium, $\mathrm{D}_{2} \mathrm{O}$ thermalizes neutrons very quickly and shifts the neutron spectrum down to below epithermal energies.

The next moderator analyzed was ${ }^{7} \mathrm{LiF}[34]$. The heavier isotope ${ }^{7} \mathrm{Li}$ does not shift the neutron spectrum down as fast as deuterium but is still very effective in slowing the neutrons on a short distance. Figure 4.12 shows the cross section of ${ }^{7} \mathrm{LiF}$. The elastic scattering resonance structure of $\mathrm{F}$ extending down to $27 \mathrm{keV}$ can be used beneficially to downscatter neutrons above this energy. The absorption and elastic cross sections of ${ }^{6} \mathrm{LiF}$ are shown in Fig. 4.13. The increasing absorption cross section of ${ }^{6} \mathrm{Li}$ for decreasing neutron energies makes this compound an excellent thermal neutron filter. In summary, 


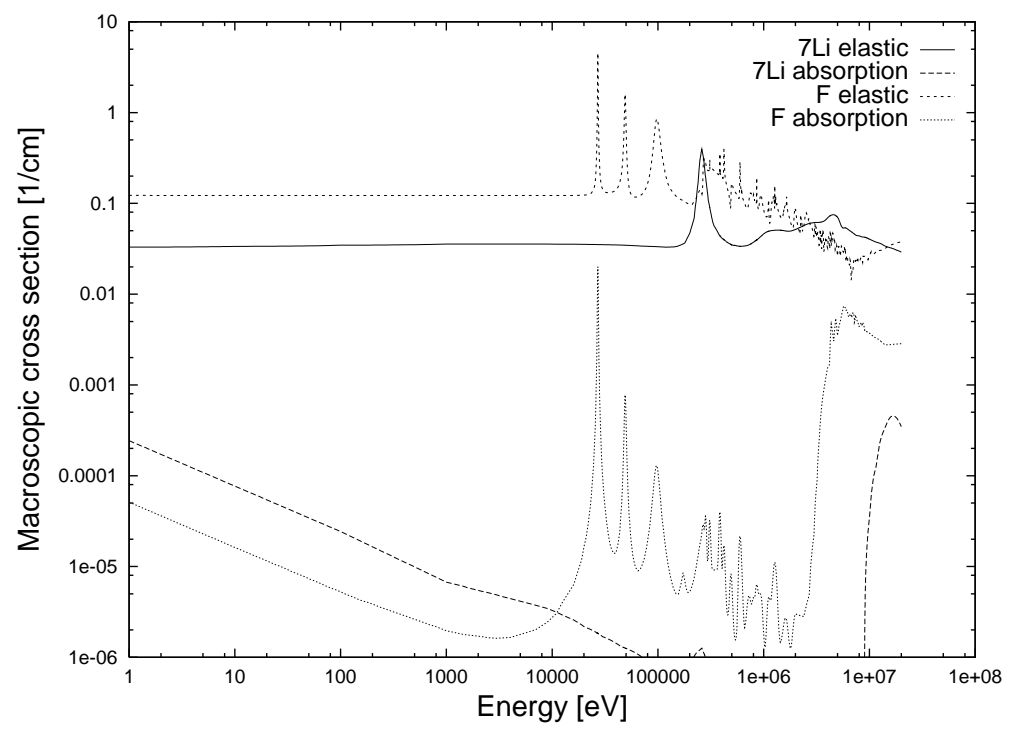

Figure 4.12: ${ }^{7} \mathrm{LiF}$ macroscopic cross sections.

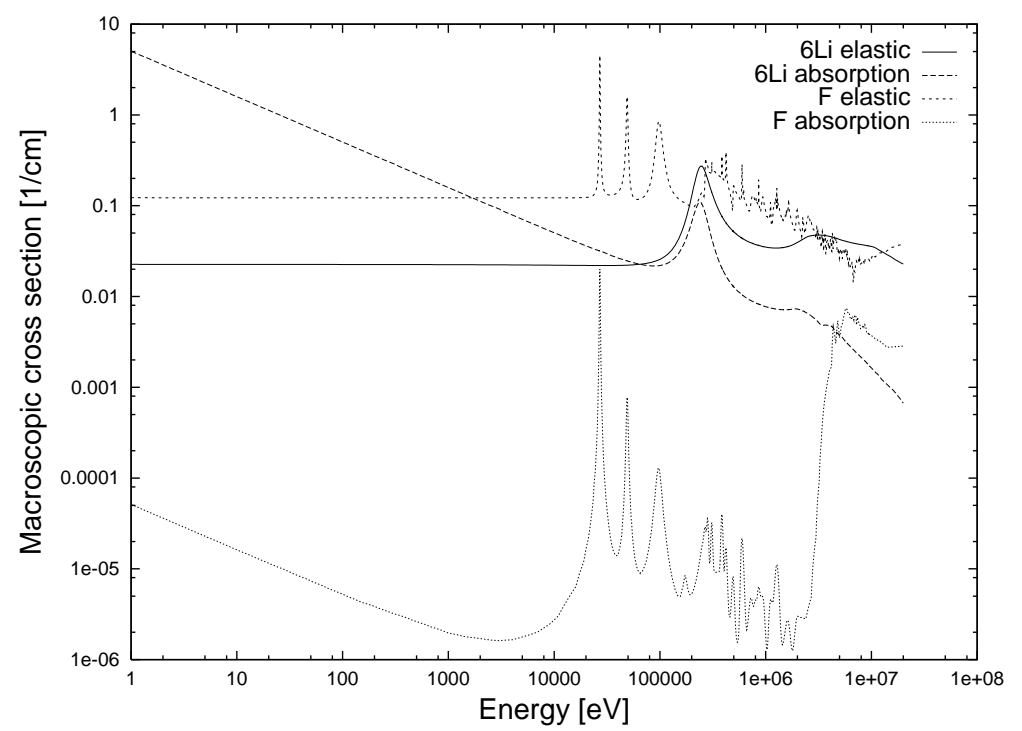

Figure 4.13: ${ }^{6} \mathrm{LiF}$ macroscopic cross sections.

lithium fluoride has the interesting properties of (a) decreasing the neutron energy in a somewhat more controllable way than $\mathrm{D}_{2} \mathrm{O}$, (b) restricting the number of neutrons above $27 \mathrm{keV}$, and (c) being a good thermal neutron filter if ${ }^{6} \mathrm{Li}$ is present. In that perspective it will be used in the later Chapters when combinations of materials will be considered.

As one can observe in Fig. 4.14, the mixture $40 \% \mathrm{Al} / 60 \% \mathrm{AlF}_{3}$ [35] is interesting 


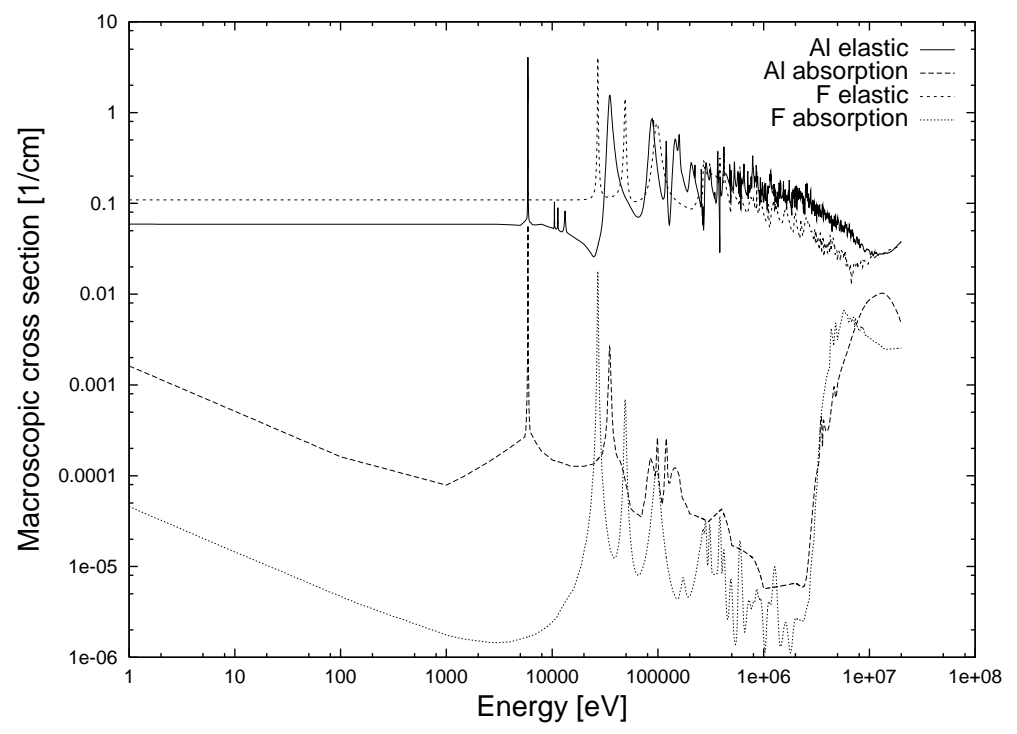

Figure 4.14: $40 \% \mathrm{Al} / 60 \% \mathrm{AlF}_{3}$ macroscopic cross sections.

in the sense that the elastic scattering resonances of Al supplement exactly the ones of $\mathrm{F}$ from $27 \mathrm{keV}$ up to the high-energy tail, except for a narrow energy range around $70 \mathrm{keV}$. This resonance structure at high energies will preferentially reduce the number of neutrons above 27 keV, see Fig. 4.14.

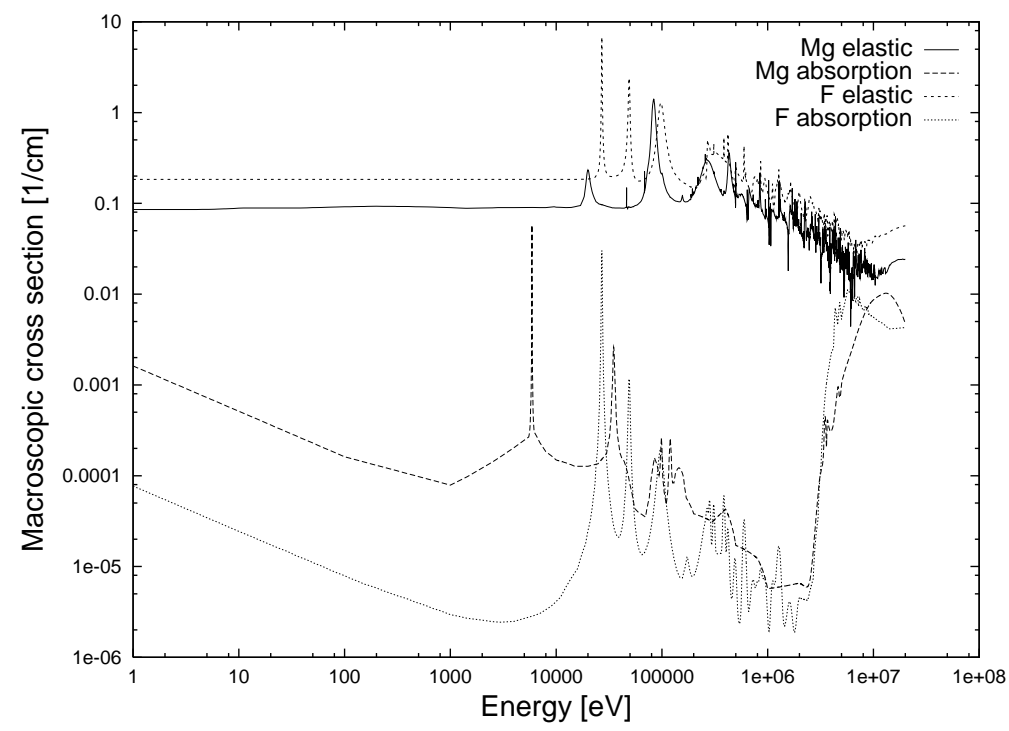

Figure 4.15: Macroscopic cross sections of $\mathrm{MgF}_{2}$.

The $\mathrm{MgF}_{2}$ (Fig. 4.15) has properties similar to the mixture $40 \% \mathrm{Al} / 60 \% \mathrm{AlF}_{3}$ but 
appeared to be worse because several energy ranges are not covered by resonances above $27 \mathrm{keV}$. It has thus been abandoned.

\subsection{Moderation of D-D neutrons}

In the first stage, ${ }^{7} \mathrm{LiF}$ and $40 \% \mathrm{Al} / 60 \% \mathrm{AlF}_{3}$ were simulated separately as moderators. Starting with $2.43-\mathrm{MeV}$ neutrons, the neutron energy distributions as a function of the BSA thickness are shown in Figs. 4.16 and 4.17. The ${ }^{7} \mathrm{LiF}$ shifts the spectrum toward

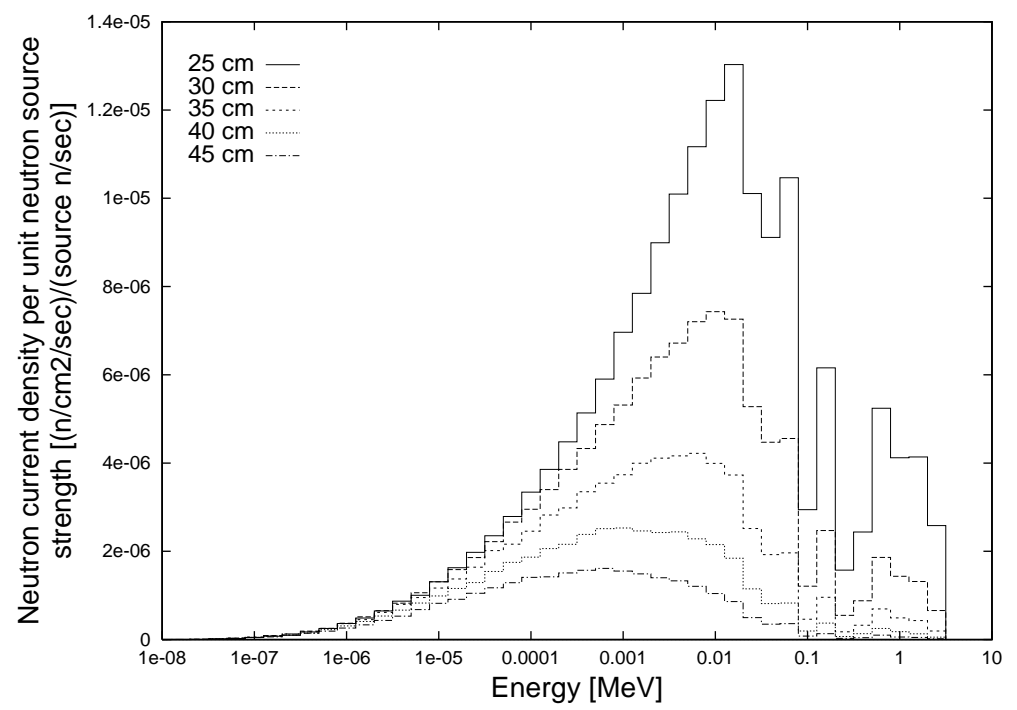

Figure 4.16: Neutron energy distribution as a function of ${ }^{7} \mathrm{LiF}$ moderator thicknesses.

lower energies, and the flux of neutrons with energies above $100 \mathrm{keV}$ is reduced to very low levels. The dips at 100 and $250 \mathrm{keV}$ in the spectra correspond to the elastic resonances of $\mathrm{F}$ and ${ }^{7} \mathrm{Li}$ respectively (see Fig. 4.12). The drawback of this moderator is that the peak is shifted to lower than $8 \mathrm{keV}$ as the moderation proceeds. The mixture $40 \% \mathrm{Al} / 60 \% \mathrm{AlF}_{3}$ exhibits a well-defined peak at $15 \mathrm{keV}$, which is close to our target energy. However, the high-energy tail of the spectra and particularly the narrow peak around $70 \mathrm{keV}-$ corresponding to energies not covered by elastic resonances in Fig. 4.14 - still remains. In summary, ${ }^{7} \mathrm{LiF}$ and $40 \% \mathrm{Al} / 60 \% \mathrm{AlF}_{3}$ could be used beneficially in combination with other moderator materials to achieve the desired epithermal energy distribution.

Several combinations of materials have been tested. The best neutron spectrum was obtained with the following combination of materials: $30 \mathrm{~cm}$ of ${ }^{7} \mathrm{LiF}, 18 \mathrm{~cm}$ 


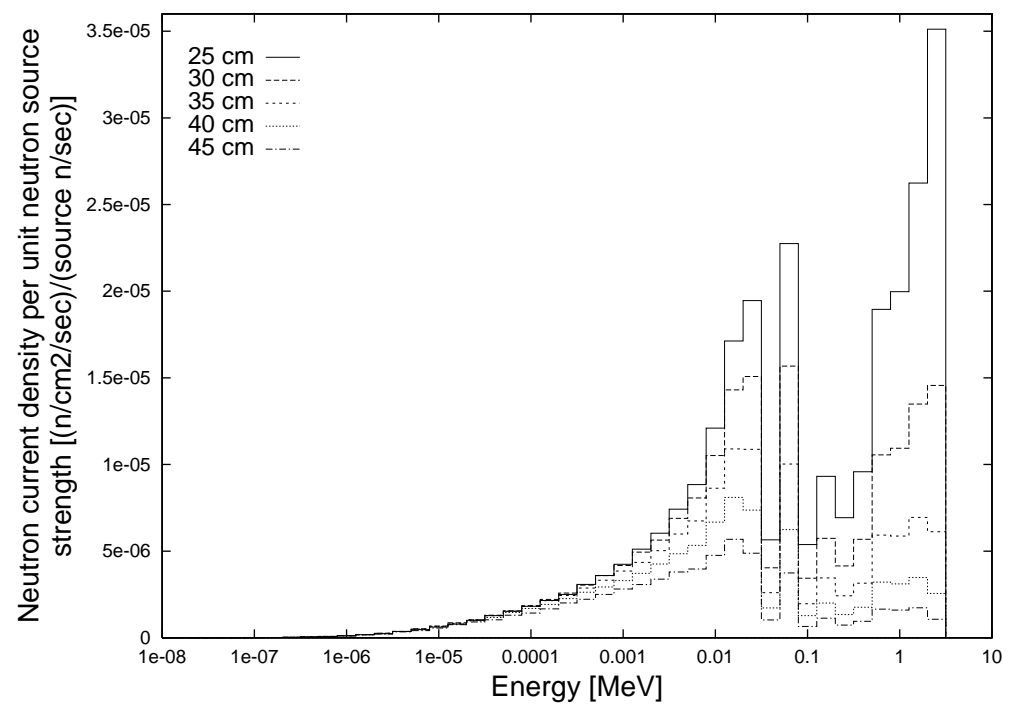

Figure 4.17: Neutron energy distribution as a function of $40 \% \mathrm{Al} / 60 \% \mathrm{AlF}_{3}$ moderator thicknesses.

of $40 \% \mathrm{Al} / 60 \% \mathrm{AlF}_{3}, 1 \mathrm{~mm}$ of ${ }^{6} \mathrm{LiF}$ - to reduce the thermal component of the neutron flux - and $1 \mathrm{~mm}$ of $\mathrm{Pb}$ - to decrease the photon flux. A 3-cm-thick layer of $\mathrm{Pb}$ between the BSA and the delimiter is used to decrease the photon dose. The neutron spectrum after moderation and the absorbed healthy-tissue dose (with its components) are shown in Figs. 4.18 and 4.19. The absorbed tumor dose to the desired depth of $8 \mathrm{~cm}$ with this

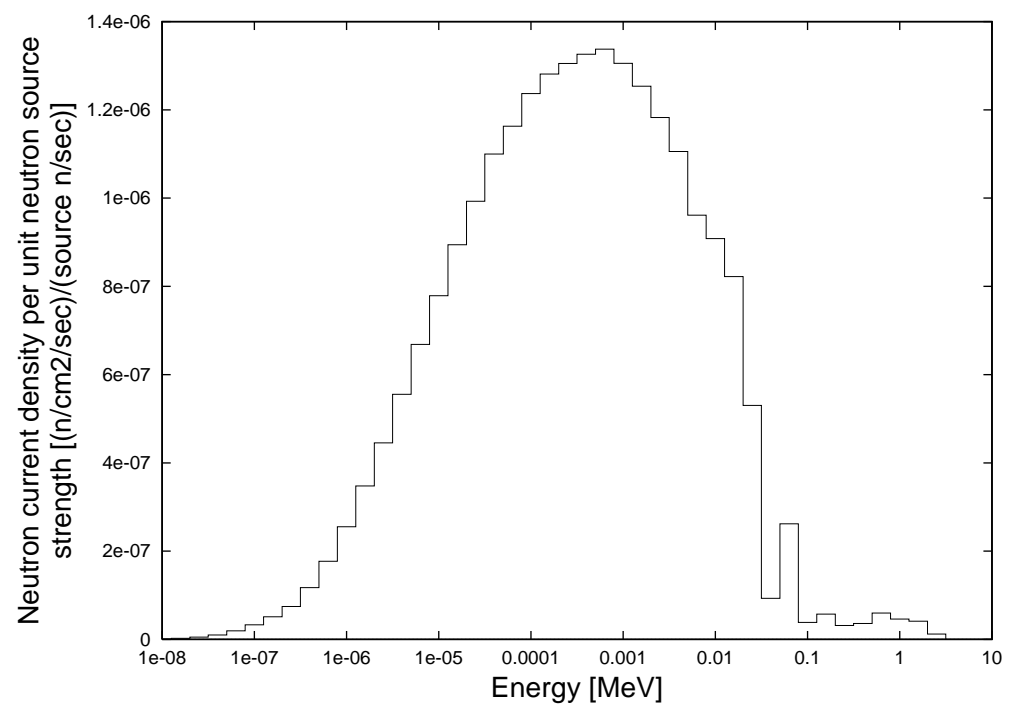

Figure 4.18: Neutron energy distribution after moderation corresponding to D-D. 


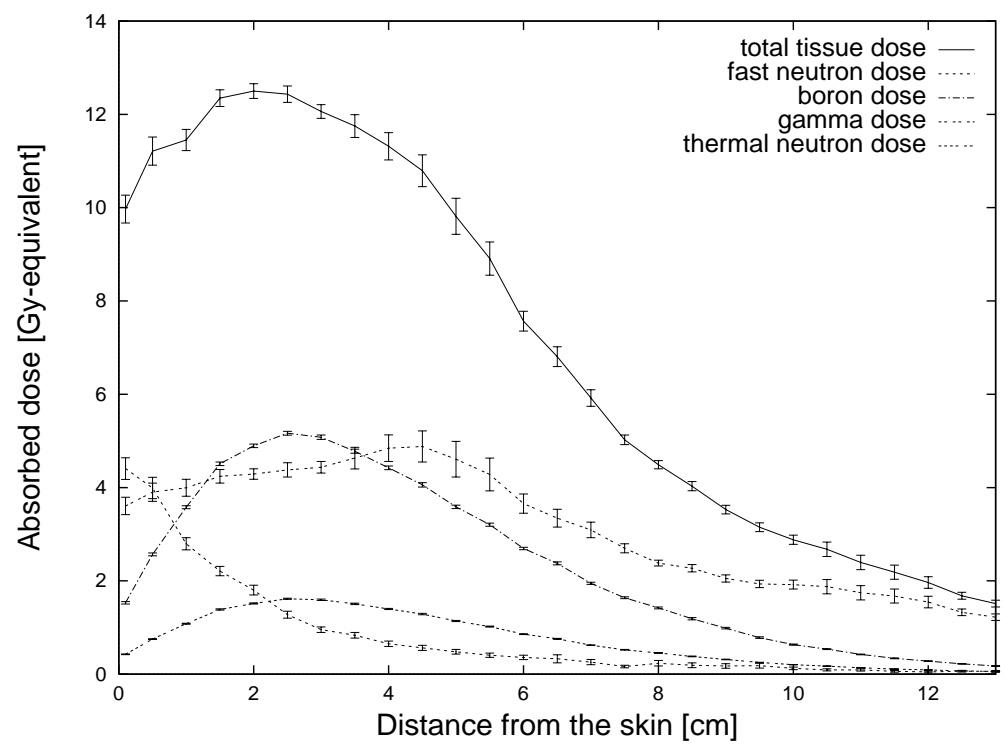

Figure 4.19: Absorbed healthy-tissue dose (with its components) corresponding to D-D.

moderation is 17.6 Gy-equivalent. The absorbed skin dose is 10.0 Gy-equivalent, which satisfies Eq. 4.4. Accounting for the neutron yield of D-D - which is $\sim 10^{9} \mathrm{n} / \mathrm{s} / \mathrm{mA}$ for a $200-\mathrm{keV}$ beam (see Sec.7.2.2) — this moderation would lead to a treatment time of $34 \mathrm{~h}$ for a 1.5-A deuteron beam. No further optimization was performed at this stage since the treatment time obtained was much larger than the maximum permissible one of $45 \mathrm{~min}$. This unacceptably large treatment time could probably be reduced by using several beams, by increasing the beam intensity, or by improving the BSA.

\subsection{Moderation of D-T neutrons}

The $\mathrm{D}$ - T reaction is a better choice when intense neutron dose is needed because its neutron yield is more than one order of magnitude higher than D-D. Figures 4.20 and 4.21 show the neutron energy distributions for different thicknesses of lead and bismuth if 14.1-MeV neutrons are used. Because of the $(n, 2 n)$ reactions, the neutron flux increases in the first $5 \mathrm{~cm}$. This neutron multiplication is slightly more pronounced for lead. Figure 4.22 shows the same graph for iron. The shapes of the spectra for lead and bismuth exhibit clear peaks at around $1 \mathrm{MeV}$, with less sharply defined peaks around 25 and $250 \mathrm{keV}$ for iron. Neutrons passing through iron are moderated to slightly lower energies than in the cases of lead and bismuth, and the 14.1-MeV ones are better suppressed. To take advantage of 


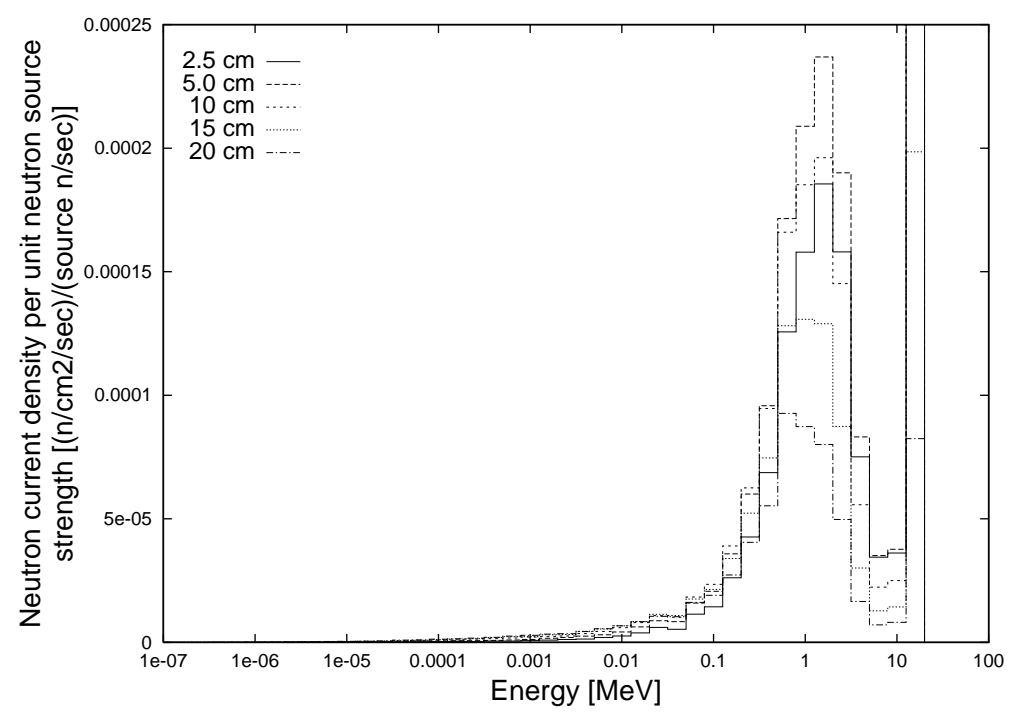

Figure 4.20: Neutron energy distribution as a function of $\mathrm{Pb}$ moderator thicknesses.

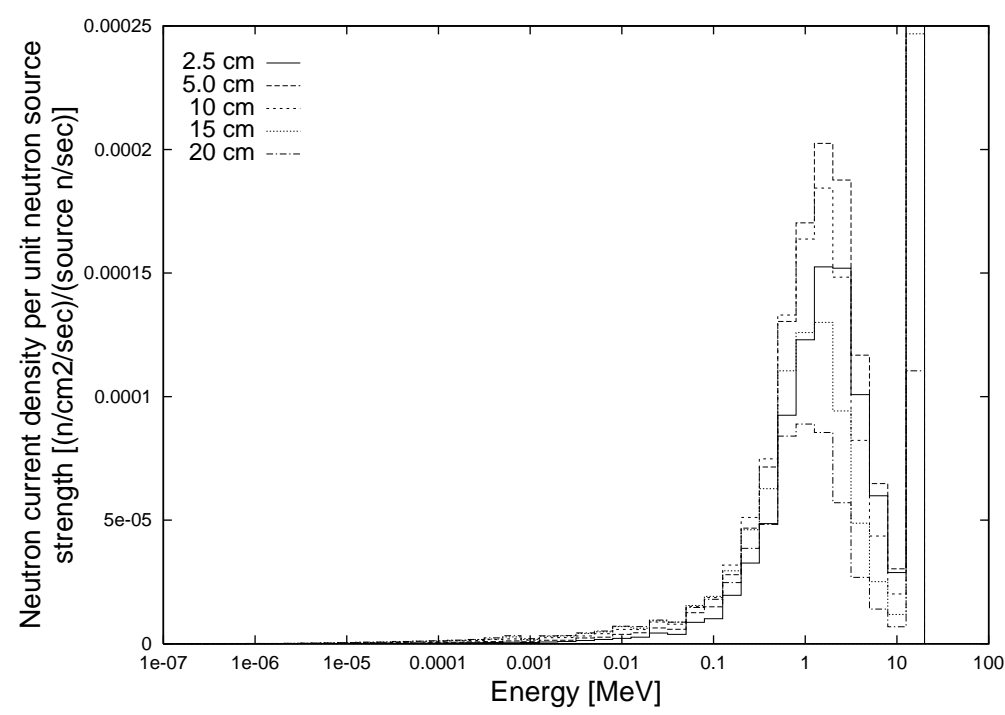

Figure 4.21: Neutron energy distribution as a function of Bi moderator thicknesses.

the properties shown in Figs. 4.20, 4.21, and 4.22, a 5-cm-thick layer of lead or bismuth, followed by a thicker layer of iron, is used at the beginning of the moderation.

The emphasis in the design of a BSA was on decreasing the high-energy neutron flux to a level as low as possible. The best D-T moderator design consisted of $5 \mathrm{~cm}$ of $\mathrm{Bi}$, $50 \mathrm{~cm}$ of $\mathrm{Fe}, 24 \mathrm{~cm}$ of $40 \% \mathrm{Al} / 60 \% \mathrm{AlF}_{3}, 1 \mathrm{~mm}$ of ${ }^{6} \mathrm{LiF}$, and $1 \mathrm{~mm}$ of $\mathrm{Pb}$. Bismuth was used to generate more neutrons with the $(n, 2 n)$ reactions. Iron was used to decrease the fast 


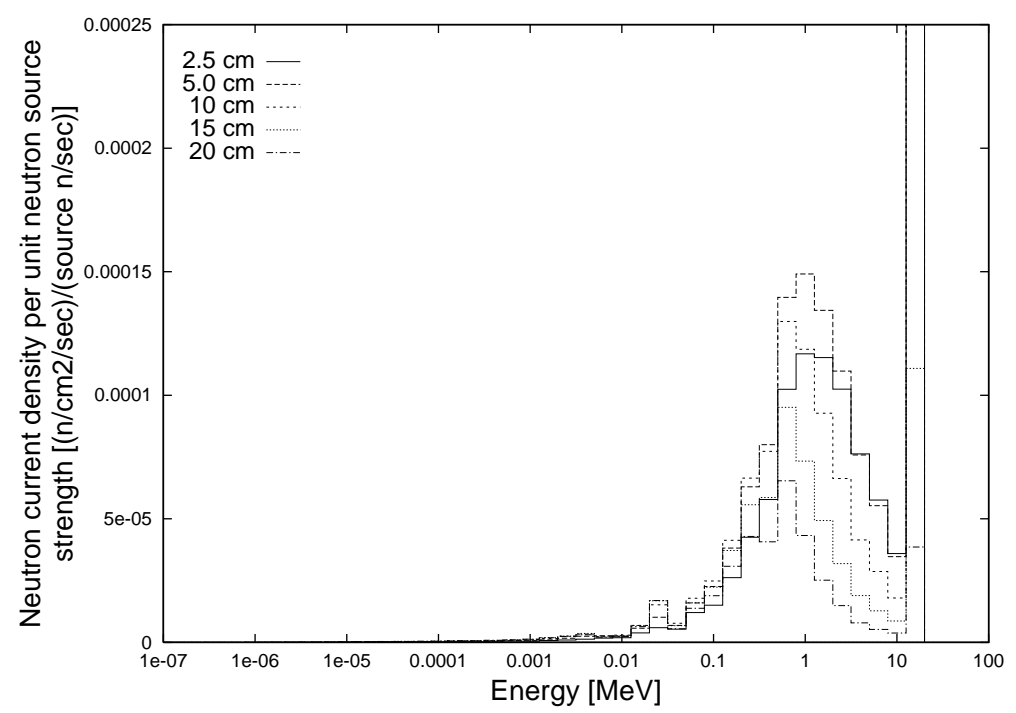

Figure 4.22: Neutron energy distribution as a function of Fe moderator thickness.

neutron flux in the range of 1 to $14 \mathrm{MeV}$, the mixture of $40 \% \mathrm{Al} / 60 \% \mathrm{AlF}_{3}$ to reduce the fast neutron flux in the range of $90 \mathrm{keV}$ and higher, and eventually the thin layers of ${ }^{6} \mathrm{LiF}$ and $\mathrm{Pb}$ were used to decrease the thermal neutron and photon fluxes, respectively. The thin layer of ${ }^{6} \mathrm{LiF}$ surrounding the BSA has been removed, as the thermal component of the neutron flux was not significant at this location. The neutron spectrum after moderation is shown in Fig. 4.23. We observe that the number of neutrons with energies greater than $3 \mathrm{MeV}$ has been reduced to about a hundredth of the number of neutrons at $15 \mathrm{keV}$. As mentioned in Sec. 3.4, it requires 20 to 40 times fewer high-energy (3 MeV and higher) neutrons than epithermal neutrons to give an identical dose to the healthy tissues. Moreover, these fast neutrons are therapeutically undesirable. This fast component of the neutron flux should be decreased by at least two or three orders of magnitude. Simulations with a 5-cm slab of lead instead of bismuth at the entrance of the moderator gave slightly worse results, especially concerning the fast neutron flux. Even though the neutron spectrum seems very promising for the dose distribution in the brain, the absorbed tumor dose at $8 \mathrm{~cm}$ is only 15.6 Gy-equivalent, and the absorbed skin dose is 11.9 Gy-equivalent. The explanation for this unexpected result is given in the next section. 


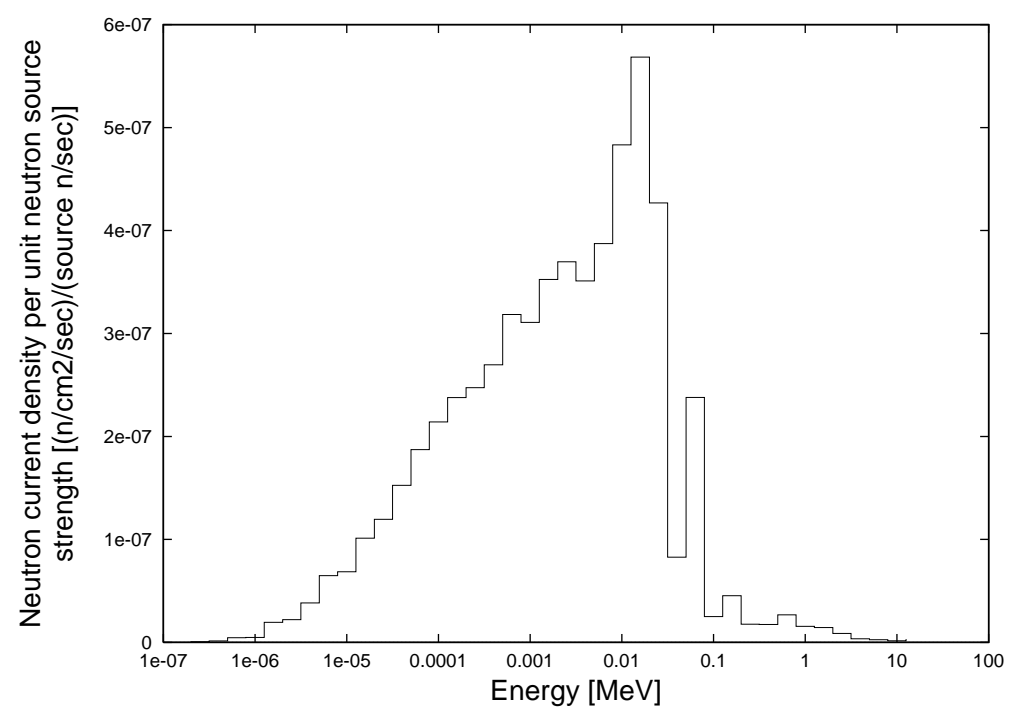

Figure 4.23: Neutron energy distribution after moderation corresponding to D-T with a 25-cm-diam BSA.

\subsubsection{Effect of the BSA diameter on the treatment characteristics}

Once a reasonably good combination of materials has been determined, the next step is to modify the diameter of the cylindrical BSA, which has been kept at $25 \mathrm{~cm}$ so far. The neutron spectrum - calculated across the 20-cm-diam exit window shown in Fig. 4.1 - corresponding to the 80 -cm-diam BSA is shown in Fig. 4.24. As one can observe, the

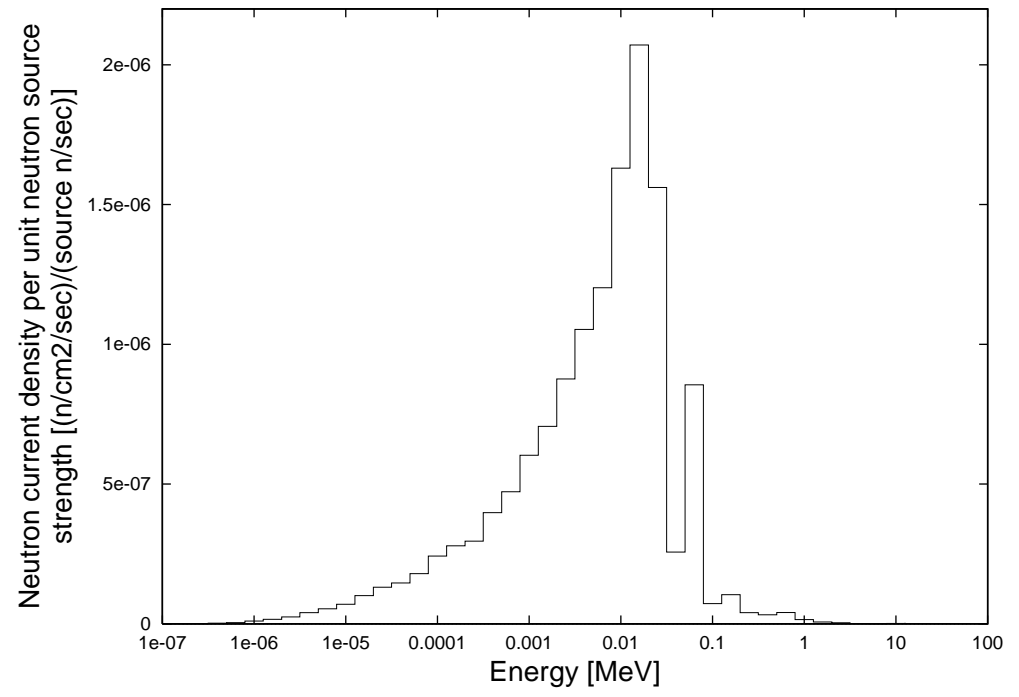

Figure 4.24: Neutron energy distribution after moderation corresponding to D-T with a 80-cm-diam BSA. 
fast neutron flux is lower, the epithermal neutron flux is higher, and the neutron energy distribution exhibits a sharper peak at $15 \mathrm{keV}$. These effects are beneficial for the treatment, except for the peak energy, which is slightly too high for the skin. Concerning the doses, these simulations with greater BSA diameters revealed the somewhat predictable trends shown in Fig. 4.25. The treatment time decreases, the absorbed tumor dose at $8 \mathrm{~cm}$ increases

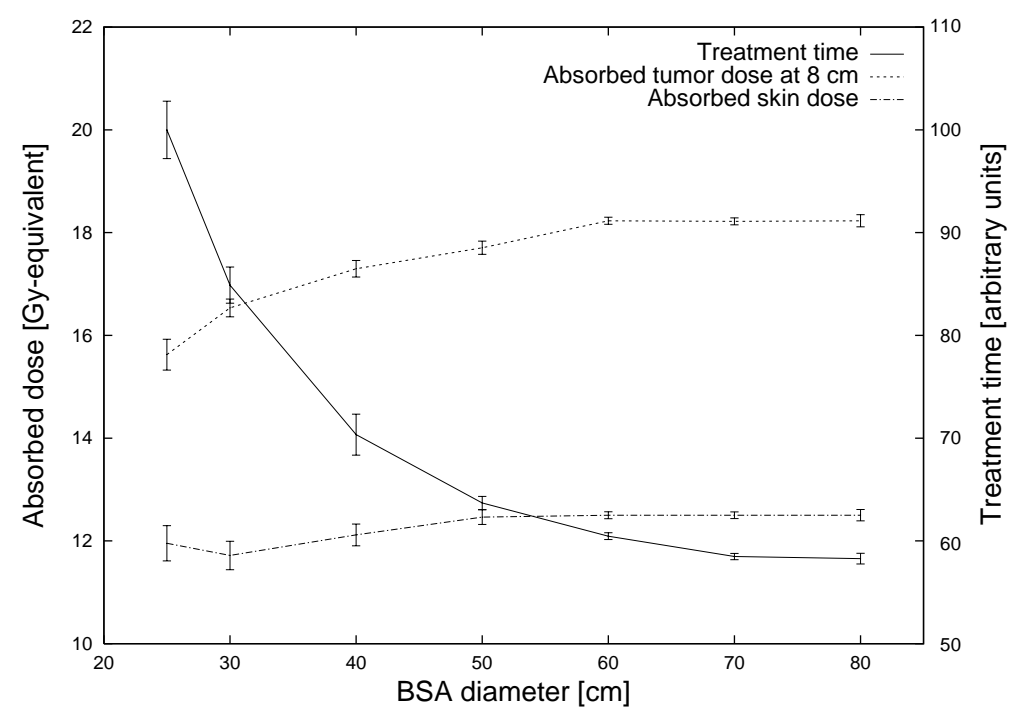

Figure 4.25: Treatment time, absorbed tumor dose at $8 \mathrm{~cm}$, and absorbed skin dose as a function of the BSA diameter, accounting for the neutrons and photons transmitted through the delimiter.

as well as the absorbed dose to the skin. For the 80 -cm-diam BSA for instance, the absorbed tumor dose at a depth of $8 \mathrm{~cm}$ is 18.2 Gy-equivalent and the absorbed skin dose is 12.5 Gy-equivalent. Among all the diameters tried, the 80 -cm-diam BSA appears to be optimal in terms of Eq. 4.2. However, Eq. 4.4 is not satisfied.

Figure 4.26 shows the same quantities as Fig. 4.25 when the neutrons and photons transmitted through the delimiter are not accounted for. Since fewer particles reach the patient's head, the treatment time is longer. For the absorbed tumor doses at $8 \mathrm{~cm}$, they are now much higher. Indeed, we have seen in Sec. 4.4.3 that the delimiter was not adequately designed to attenuate photons. Therefore, the additional photons transmitted through the delimiter increase the photon dose contribution to the healthy tissues. As a result, the boron dose contribution has to decrease in the entire brain - and particularly at the point of maximum healthy-tissue dose — to satisfy the 12.5 Gy-equivalent limit for healthy tissue. From Eq. 3.8, we see that the absorbed tumor dose is significantly reduced by the decrease 


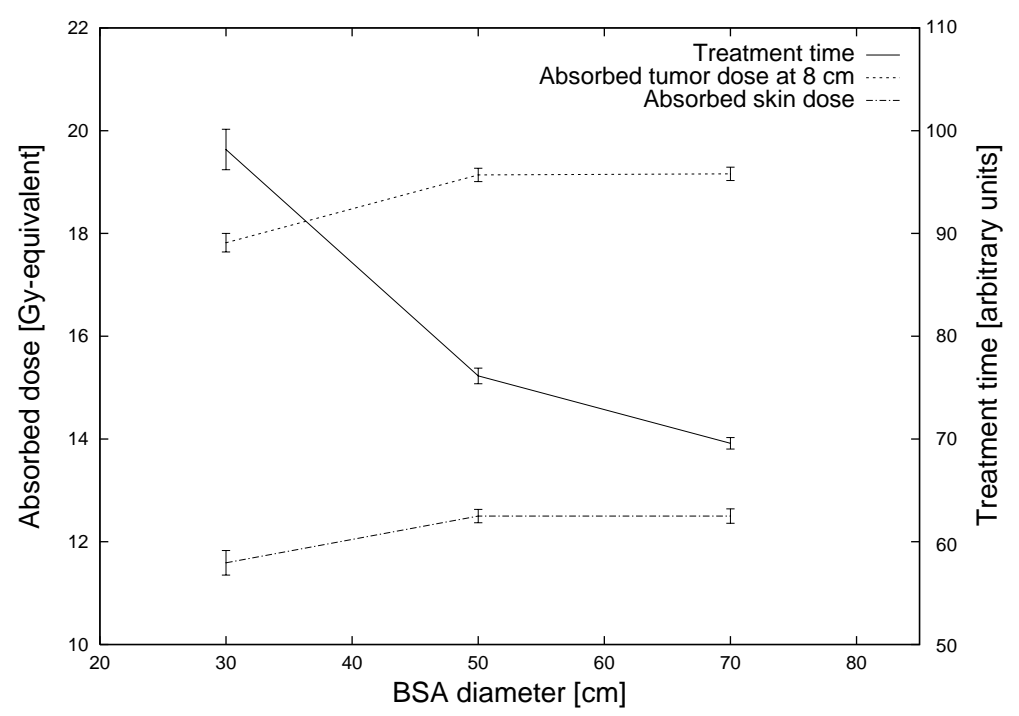

Figure 4.26: Treatment time, absorbed tumor dose at $8 \mathrm{~cm}$, and absorbed skin dose as a function of the BSA diameter, not accounting for the neutrons and photons transmitted through the delimiter.

in boron dose, which is hardly compensated by the increase in photon dose $(R B E \gamma=1$ versus $C F=3.8$ ).

The optimization study was mostly based on trying to shape the neutron spectrum across the exit window. Since the neutron spectra obtained peak around $10 \mathrm{keV}$, one can expect relatively high tumor doses. However, the high tumor doses shown in Fig. 4.26 represent in fact the doses that would have been obtained with the neutron and photon spectra across this exit window only, without taking into account particles transmitted through the delimiter. By taking into account the particles transmitted through the delimiter, the actual tumor doses were much lower due to the maximum healthy-tissue dose constraint, as shown in Fig. 4.25. To reduce this contamination by undesired photons transmitted through the delimiter, a thick lead shield is added between the BSA and the delimiter.

\subsubsection{Reflector material and thickness}

In this section, we try to determine which materials have the best reflective properties for high-energy neutrons. Different reflector materials - such as lead, aluminum oxide, and graphite - and reflector thicknesses are simulated to determine their reflective capabilities. The BSA used for these simulations has a diameter of $80 \mathrm{~cm}$, and its composition is slightly changed to reduce the dose to the skin. In Fig. 4.27, the treatment time is 


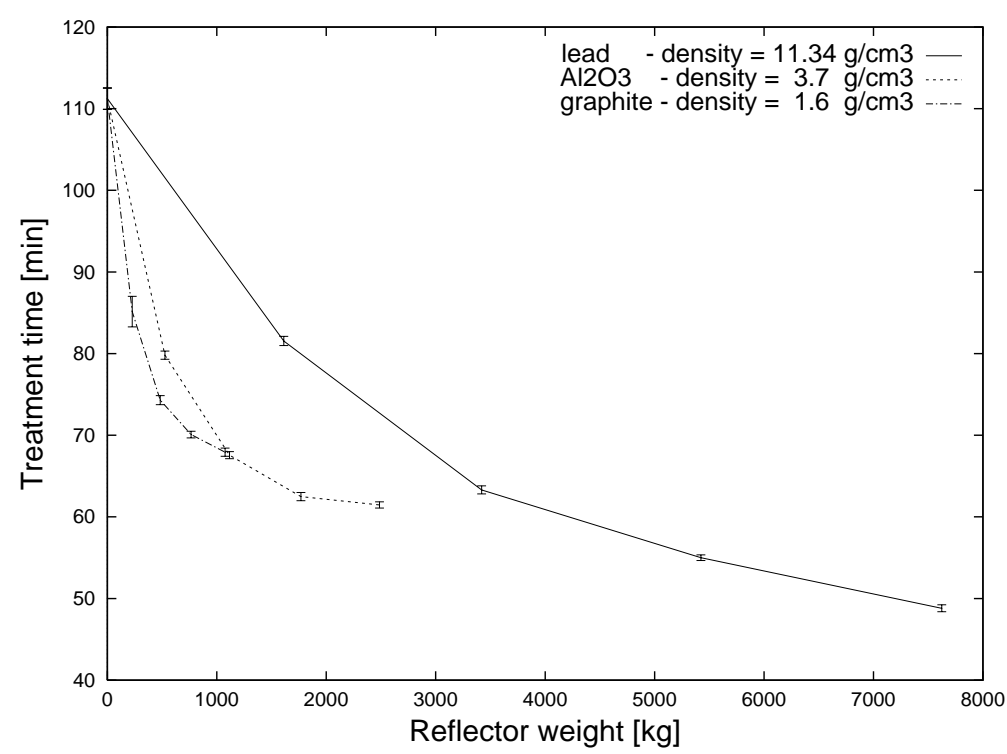

Figure 4.27: Treatment time versus reflector weight. A neutron source strength of $10^{14} \mathrm{n} / \mathrm{s}$ is assumed.

estimated as a function of the reflector weight. For each material, simulations with five different reflector thicknesses are performed, from 0 to $20 \mathrm{~cm}$ thick in increments of $5 \mathrm{~cm}$. The treatment time decreases more rapidly for the lower density materials, but the decrease seems to approach asymptotically a higher value. The absorbed dose to the tumor at $8 \mathrm{~cm}$

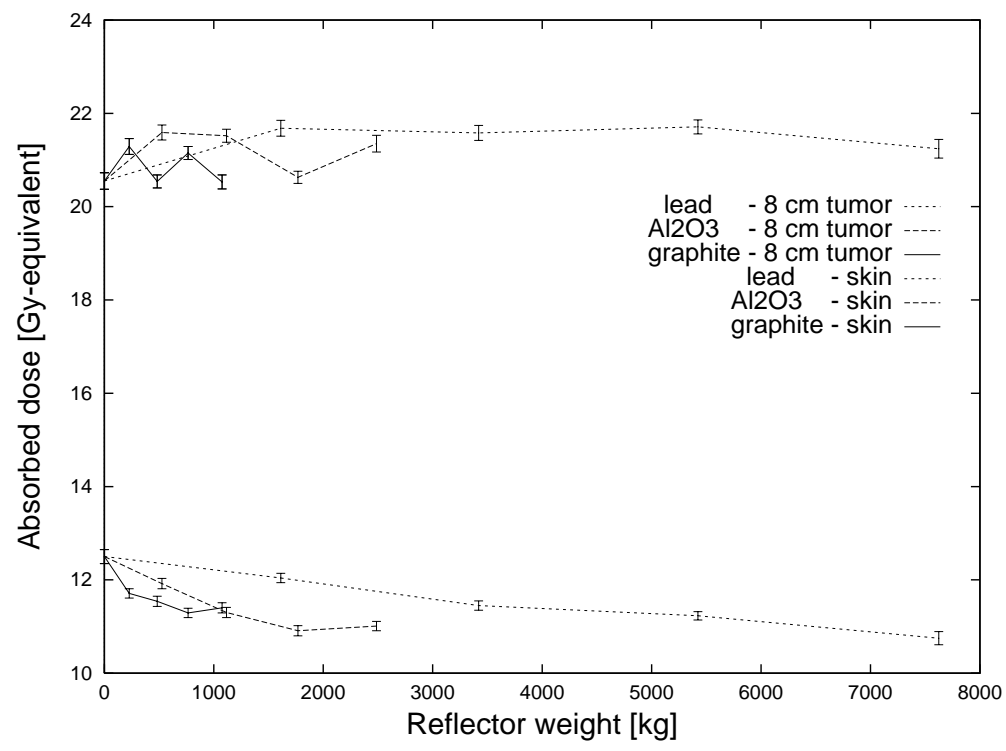

Figure 4.28: Absorbed tumor dose at a depth of $8 \mathrm{~cm}$ and absorbed skin dose as a function of the reflector weight. 
and the absorbed skin dose are represented as a function of the reflector material and weight in Fig. 4.28. For all materials considered, the absorbed skin dose decreases as the reflector thickness increases. Concerning the absorbed tumor dose, lead seems to perform slightly better than aluminum oxide and graphite.

Several conclusions can be drawn from this section. Treatment characteristics always improve with reflector thickness, regardless of the material. If the reflector weight is limited to less than $1000 \mathrm{~kg}$, the lower density materials such as graphite and aluminum oxide are preferred over lead. They lead to lower treatment times and lower absorbed skin dose. If weight is not a concern however, lead gives better results for large reflector thicknesses.

\subsubsection{Optimal BSA for D-T neutrons}

The best results, among the designs that the author has analyzed and in terms of Eqs. 4.2- 4.5, were obtained for the 80-cm-diam moderator design consisting of $5 \mathrm{~cm}$ of $\mathrm{Bi}, 34 \mathrm{~cm}$ of $\mathrm{Fe}, 39 \mathrm{~cm}$ of the mixture $40 \% \mathrm{Al} / 60 \% \mathrm{AlF}_{3}, 1 \mathrm{~mm}$ of ${ }^{6} \mathrm{LiF}$ and $1 \mathrm{~mm}$ of $\mathrm{Pb}$. Bismuth was used to generate more neutrons with the $(n, 2 n)$ reactions. Iron could decrease the fast neutron flux in the range of 1 to $14 \mathrm{MeV}$. The mixture of $40 \% \mathrm{Al} / 60 \% \mathrm{AlF}_{3}$ could reduce the fast neutron flux in the range of $90 \mathrm{keV}$ and higher, and eventually the thin layers of ${ }^{6} \mathrm{LiF}$ and $\mathrm{Pb}$ could decrease the thermal neutron and photon fluxes, respectively. The BSA was surrounded by a 20 -cm-thick $\mathrm{Pb}$ reflector. A 3-cm-thick layer of lead is added between the BSA and the delimiter around the exit window to attenuate the photons. A 2cm-thick lead liner is placed inside the delimiter at the exit window. Numerical simulations showed that this collimator reduces the treatment time and enhances the absorbed tumor dose at $8 \mathrm{~cm}$. One could argue that the presence of this collimator increases the neutron beam diameter from 12 to $16 \mathrm{~cm}$, because lead does not attenuate neutron flux as efficiently as the delimiter. The increase in tumor dose would then be due solely to the increase in diameter (see Table 3.1). However, Figs. 4.29a and 4.29b show that the absorbed healthytissue and tumor dose distributions for this BSA are very similar to the ones shown in Figs. 3.6, which correspond to the case of an ideal 12-cm-diam neutron beam. The presence of the collimator does not alter the dose distributions in the brain, nor does it increase significantly the volumes where the doses to the healthy tissues are large. Therefore, it effectively improves the neutron beam quality. The neutron spectrum (across the exit 


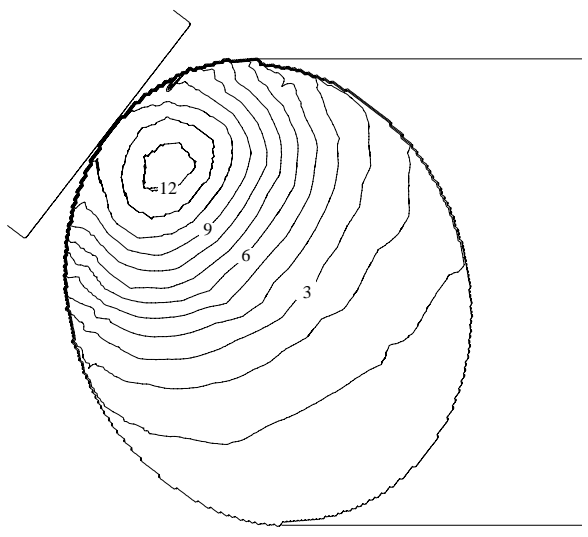

(a)

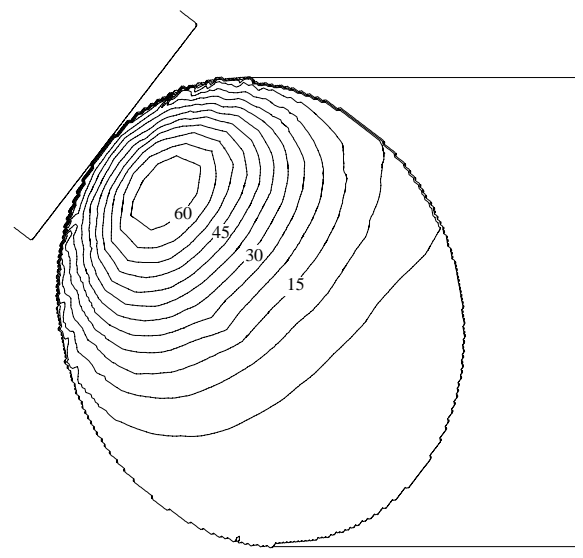

(b)

Figure 4.29: Isodose countour plots of the absorbed (a) healthy-tissue and (b) tumor dose distributions for the neutron beam obtained with the optimal BSA.

window) and dose distributions as a function of depth corresponding to this BSA are shown in Figs. 4.30, 4.31, and 4.32. The absorbed tumor and skin doses are 24.2 and 10.2 Gyequivalent, respectively.

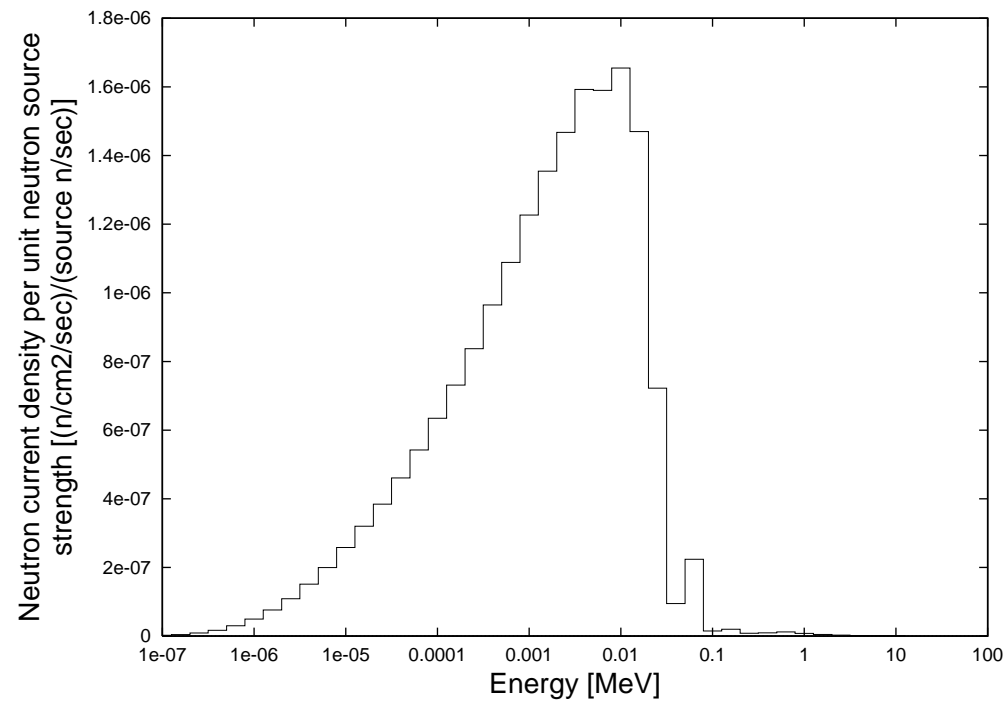

Figure 4.30: Neutron energy distribution after moderation corresponding to the optimal BSA for D-T.

For a deuteron beam with energy of $150 \mathrm{keV}$ bombarding a thick TiT 1.6 $_{1}$ target, a neutron yield of $1.12 \times 10^{14} \mathrm{n} / \mathrm{s} \cdot A$ can be estimated theoretically, see Sec. 7.2.2. For our BSA design, a 1.5-A beam intensity would lead to a treatment time of 21 min using 


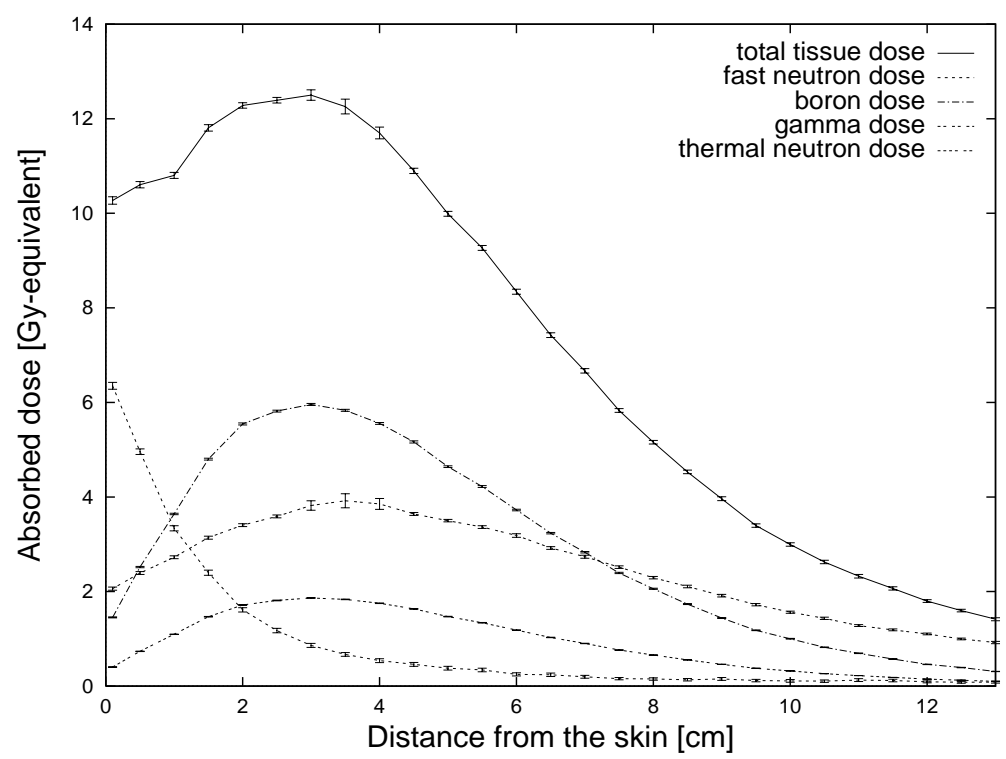

Figure 4.31: Absorbed healthy-tissue dose (with its components) corresponding to the optimal BSA for D-T.

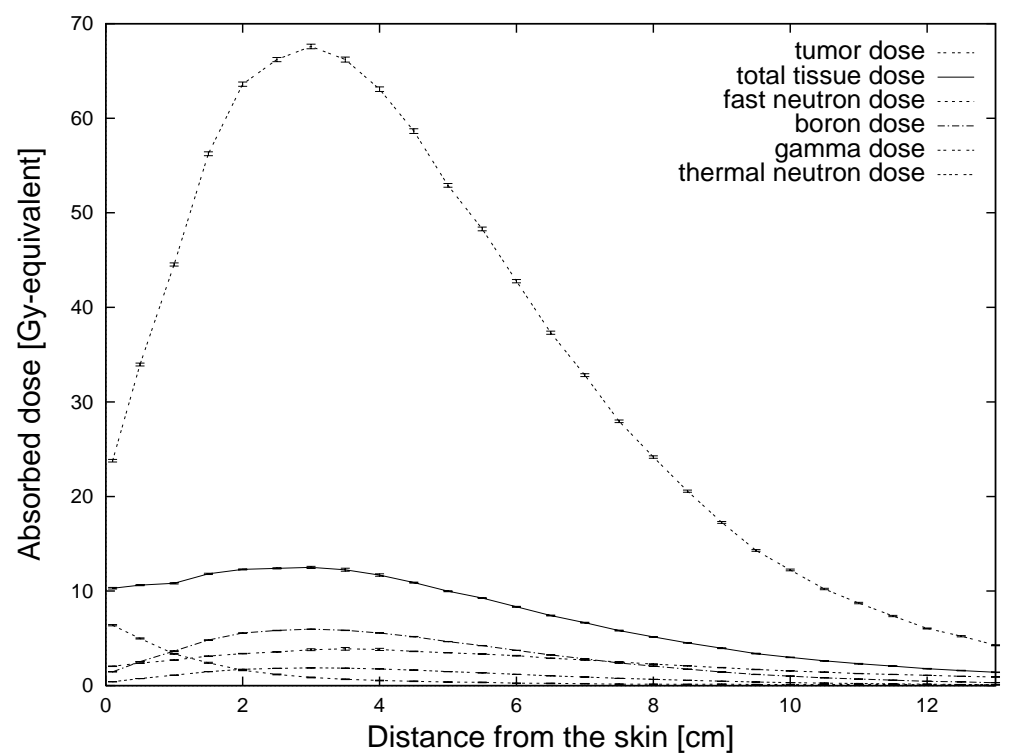

Figure 4.32: Absorbed tumor dose corresponding to the optimal BSA for D-T.

a single beam. The power load on the 5-cm-diam water-cooled target would be much too high $\left(11.4 \mathrm{~kW} / \mathrm{cm}^{2}\right)$ in that case. With a $30 \times 30 \mathrm{~cm}^{2}$ target, the power load is $255 \mathrm{~W} / \mathrm{cm}^{2}$, which is technologically feasible. MCNP simulations of the BSA and the phantom head show that the treatment characteristics are not affected significantly by the larger size of 
the target. Another issue is the target lifetime, which is dictated by the replacement of tritium by deuterium in the target during operation. Typical lifetimes for tritiated targets are $1 \mathrm{~mA} \cdot \mathrm{h} / \mathrm{cm}^{2}$ or just over half an hour for the $30 \times 30 \mathrm{~cm}^{2}$ target. To enhance the lifetime of the target and to maintain a constant neutron output, a mixed $50 \%$ deuteron/ $50 \%$ triton beam will be used instead of a $100 \%$ deuteron beam to load a drive-in target. With mixed beam operation, the neutron output per unit power is decreased by approximately a factor of 2 , but the target lifetime is now only limited by sputtering of the titanium/scandium layer. One can estimate a neutron yield of $5.0 \times 10^{13} \mathrm{n} / \mathrm{s} \cdot A$ in mixed beam operation (see Sec. 7.2.3), assuming $85 \%$ monatomic and $15 \%$ molecular species in the beam. This mode of operation would lead to a treatment time of $47 \mathrm{~min}$ for a 1.5-A mixed beam.

\begin{tabular}{|l|c|c|c|c|c|}
\hline Neutron source & ${ }^{3} \mathrm{H}(\mathrm{d}, n)^{4} \mathrm{He}$ & ${ }^{2} \mathrm{H}(\mathrm{d}, \mathrm{n})^{3} \mathrm{He}$ & ${ }^{7} \mathrm{Li}(\mathrm{p}, \mathrm{n})^{7} \mathrm{Be}$ & ${ }^{7} \mathrm{Li}(\mathrm{p}, n)^{7} \mathrm{Be}$ & $\mathrm{BMRR}$ \\
\hline \hline Moderator & $\begin{array}{c}\mathrm{Bi} / \mathrm{Fe} \\
\mathrm{Al} / \mathrm{AlF} \mathrm{F}_{3}\end{array}$ & $\begin{array}{c}{ }^{7} \mathrm{LiF} \\
\mathrm{Al} / \mathrm{AlF} \mathrm{F}_{3}[36]\end{array}$ & $\begin{array}{c}{ }^{7} \mathrm{LiF} \\
{[10]}\end{array}$ & $\begin{array}{c}\mathrm{Al} / \mathrm{AlF}_{3} \\
{[10]}\end{array}$ & $\begin{array}{c}\left(\mathrm{Al} \mathrm{I}_{2} \mathrm{O}_{3}\right) \\
{[30]}\end{array}$ \\
\hline $\begin{array}{l}\text { Proton or deuteron } \\
\text { energy [MeV] }\end{array}$ & 0.15 & 0.2 & 2.3 & 2.4 & - \\
\hline $\begin{array}{l}\text { Proton or deuteron } \\
\text { current [m A] }\end{array}$ & 1500 & 1500 & 20 & 20 & $(3 \mathrm{MW})$ \\
\hline Treatment time [min] & 47 & 2040 & 40 & 54 & 39 \\
\hline $\begin{array}{l}\text { Absorbed tumor dose } \\
\text { (max) [Gy-equivalent] }\end{array}$ & 67.6 & 60.2 & 64.3 & 65.1 & 61.6 \\
\hline $\begin{array}{l}\text { Absorbed tumor dose } \\
(5 \mathrm{~cm}) \text { [Gy-equivalent] }\end{array}$ & 52.9 & 43.0 & 50.5 & 51.4 & 38.6 \\
\hline $\begin{array}{l}\text { Absorbed tumor dose } \\
(8 \mathrm{~cm}) \text { [Gy-equivalent] }\end{array}$ & 24.2 & 17.6 & 21.4 & 22.3 & 14.5 \\
\hline Advantage depth [cm] & 9.9 & 9.0 & 9.5 & 9.5 & 8.4 \\
\hline
\end{tabular}

Table 4.2: Comparison of treatment characteristics for different neutron sources.

In Table 4.2, the treatment characteristics obtained with the D-T neutron source are compared with those obtained with other neutron sources. From the point of view of the dose distributions, the optimized spectrum from the D-T neutron source produces about $65 \%$ higher dose near the center of the brain than the currently used reactor spectrum at BMRR [30].

Taking advantage of the small sizes of the ion source and accelerator for D-T, two beams could easily be used in parallel. By increasing the beam intensity and the number of beams, treatment times could be reduced and treatment characteristics could be improved. 


\subsection{Summary of results}

Based on the results of the ideal neutron beam simulation study, the fusion reactions D-D and D-T were then investigated as neutron sources for BNCT. Two different methods were used to compute the dose distribution in the brain. The first method was based on the simulation of the radiation transport in the BSA and the phantom with MCNP. The second method is based on the coupling of MCNP and BNCT_RTPE for the radiation transport simulations in the BSA and phantom, respectively, the coupling being done through an exit window between the BSA and the phantom. The radiation transmitted through the delimiter is not accounted for in the second method, due to the limitations of BNCT_RTPE, which makes it less accurate than the first one. The first method, although more accurate, is much more time consuming due to the use of MCNP to simulate radiation transport through both the BSA and the phantom head.

Our analysis shows that the low neutron yield of the D-D reaction is an obstacle for the treatment of glioblastoma multiforme. On the other hand, high-energy neutrons from $\mathrm{D}-\mathrm{T}$ can be moderated to the desired energy range without reducing the neutron flux to a negligible level. With the optimal moderator and lead reflector configuration, a 1.5-A mixed deuteron/triton beam with energy of $150 \mathrm{keV}$ accelerated onto a titanium target leads to a treatment time of $\sim 45$ min. As observed in Table 4.2 , the dose near the center of the brain obtained with this configuration is more than $65 \%$ higher than doses obtained by a neutron beam currently used at BMRR for clinical trials, and it is comparable to other

accelerator-based neutron sources. A multiple-beam configuration could increase the tumor dose at the center of the brain and reduce the treatment time. 


\section{Chapter 5}

\section{Neutron beam design for boron neutron capture synovectomy}

\subsection{Introduction}

Rheumatoid arthritis is a disease characterized by the inflammation of the synovial membrane or synovium, a thin tissue layer that overlays articulating joints (such as knee and finger joints) and provides lubrication for the articulation. It results in swollen, inflamed, and painful joints that can cause loss of joint function at advanced stages. Different treatments are currently used for this disease. The most common is the administration of drugs to reduce synovial inflammation. Even though the drugs work successfully for the majority of the patients, some joints are unresponsive to this treatment, and removal of the synovial membrane becomes necessary. Excision of the inflamed synovium via invasive surgery is effective in treating the disease but presents some dangers, such as infection, hemorrhage and anesthesia. Moreover, complete removal of the inflamed tissues is technically difficult because of the recesses and crevices of the joints. This synovectomy technique also incapacitates the patient during the recovery period. Radiation synovectomy using beta-emitting radionuclides presents several advantages, such as success rates comparable to surgery, local anesthesia, no rehabilitation time, lower cost, and a less time-consuming procedure. However, irradiation of healthy tissues by diffusion of beta-emitters away from the joint is a major concern $[37,38]$.

Boron neutron capture synovectomy (BNCS) $[5,11,39,40]$ is currently being in- 
vestigated as an alternative approach for treatment for rheumatoid arthritis. The treatment is similar to boron neutron capture therapy [2,41], presently being studied for treatment of glioblastoma multiforme, a malignant brain tumor. It employs the ${ }^{10} \mathrm{~B}(n, \alpha)^{7} \mathrm{Li}$ reaction. One of the applications of BNCS is the treatment of diseased knee joints. A boronated compound such as $\mathrm{K}_{2} \mathrm{~B}_{12} \mathrm{H}_{12}$ is injected into the synovial membrane of the diseased knee. After injection, the knee is exposed to a low-energy neutron beam. Boron-10 atoms, with their large absorption cross section for thermal neutrons, undergo fission reactions, releasing high-energy, high-LET alpha particles and lithium nuclei. These particles deposit their energy locally (typically 2.3 to $2.8 \mathrm{MeV}$ within 4 to $9 \mu \mathrm{m}$ ), and they damage or kill cells along their paths. Since boron has been previously concentrated in the synovium cells, the dose given to the synovium will be significantly higher than the dose given to healthy tissues and bone. The treatment is expected to take only a few minutes of neutron irradiation time. BNCS offers the same advantages as therapies based on beta-emitting radionuclides by being noninvasive. Additionally, it permits a better control on the irradiation of healthy tissues, since cell killing is triggered by the neutron beam and stops after irradiation.

\subsection{Clinical requirements}

To design an efficient, practical, and safe neutron beam for radiotherapy, it is necessary to determine clinical criteria. To be efficient, the neutron beam must deliver to the boron-loaded synovium a dose sufficient to thoroughly kill the cells of the inflamed tissues. The dose required to produce a clinical effect on the synovium, as estimated for beta-emitters, is about 100 Gy-equivalent. Boronated compounds uptake in the synovium higher than $1000 \mathrm{ppm}$ have been reported [5] in the past few years. With this localized high boron uptake, high doses to the synovial membrane can be achieved without irradiating neighboring tissues excessively.

Skin and bone are of concern. Radiation effects in the skin are nonstochastic, and a mild skin reddening, which is not permanent, is observed at doses of approximately 8 Gyequivalent [19]. Concerning the bone, potential radiation effects are stochastic, indicating that the probability of cancer induction increases with the radiation dose without dose threshold [39]. Reduction of bone dose is crucial to the success of any BNCS treatment regimen.

Let $\mathrm{D}_{s y n}, \mathrm{D}_{s k}$ and $\mathrm{D}_{b o n e}$ be the average absorbed synovium, skin, and bone doses 
per neutron emitted by the source. Let $\mathrm{N}$ be the number of source neutrons required to reach the 100 Gy-equivalent dose on the synovium. The synovium, skin, and bone doses are then given by $\mathrm{N} \cdot \mathrm{D}_{s y n}, \mathrm{~N} \cdot \mathrm{D}_{s k}$, and $\mathrm{N} \cdot \mathrm{D}_{\text {bone }}$, respectively. In mathematical terms, the neutron beam energy $\mathrm{E}_{\text {optimal }}$ is optimal when

$$
D_{\text {bone }}\left(E_{\text {optimal }}\right)=\min \left(D_{\text {bone }}(E)\right)
$$

for $0 \leq E \leq 14 \mathrm{MeV}$, under the constraints

$$
N \cdot D_{s k}\left(E_{\text {optimal }}\right) \leq 8 \quad \text { Gy-equivalent }
$$

and

$$
N \cdot D_{\text {syn }}\left(E_{\text {optimal }}\right)=100 G y-\text { equivalent. }
$$

The number of neutrons $\mathrm{N}$ is determined using Eq. 5.3. All the doses are scaled in such a way that Eq. 5.3 is verified. From Eq. 5.3, one can compute the time T required for treatment using

$$
T=\frac{N}{S}[s]
$$

where $S$ is the neutron source strength in $n / s$.

An analysis based on two figures-of-merit to measure the beam quality has been proposed by Yanch [39]. The first one is the ratio of the synovium absorbed dose to the skin absorbed dose. To satisfy both the 100-Gy-equivalent dose to the synovium and the 8-Gy-equivalent dose limit on the skin, this ratio should be greater than 12.5 . The second is the ratio of the synovium absorbed dose to the bone absorbed dose. It is advantageous to maximize this ratio to limit potential cancer induction. In terms of the quantities defined earlier, Yanch et al.'s analysis results in the following equations for the optimization. The neutron energy $\mathrm{E}_{\text {optimal }}$ is optimal when

$$
D_{\text {syn }}\left(E_{\text {optimal }}\right) / D_{\text {bone }}\left(E_{\text {optimal }}\right)=\max \left(D_{\text {syn }}(E) / D_{\text {bone }}(E)\right)
$$

for $0 \leq E \leq 14 \mathrm{MeV}$, under the constraint

$$
D_{\text {syn }}\left(E_{\text {optimal }}\right) / D_{\text {sk }}\left(E_{\text {optimal }}\right) \geq 100 / 8=12.5 \text {. }
$$

Besides maximizing the ratio in Eq. 5.5 under the constraint of Eq. 5.6, an ideal neutron beam must have sufficient intensity for short treatment times, although fractionated radiation schemes could be adopted. This second criterion will be used in Chapter 6 for the design of beam-shaping assemblies. 


\subsection{Dose computations in the knee}

The human knee model used in our numerical simulation is taken from Ref. [11] and is shown in Fig. 5.1. It is composed of concentric circles representing different layers

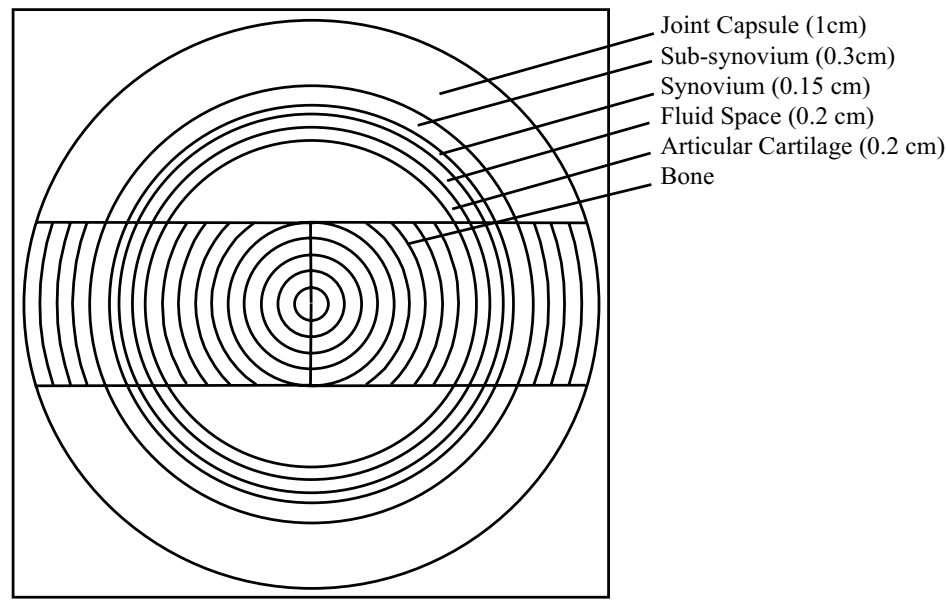

Figure 5.1: Cross-section view of the cylindrical knee model used in the MCNP simulations. Absorbed doses are computed as a function of the depth within the 2.5-cm-diam cylinder intersecting the different tissue layers.

of tissue at various depths. The outer diameter of the knee is $8.7 \mathrm{~cm}$ and corresponds to the approximate width of an adult human knee. Tissue layers were assigned the following thicknesses estimated using magnetic resonance imaging data of the knee: 1.0-cm joint capsule, 0.3-cm subsynovium, 0.15-cm synovium, 0.2-cm fluid space, 0.2-cm articular cartilage, and 5.0-cm-diam bone. In the knee model, the synovium layer was therefore at the depth of 1.3 to $1.45 \mathrm{~cm}$ below the surface of the skin. The estimates for elemental compositions of tissue and bone are shown in Table 5.1. Boron-10 concentrations of $1000 \mathrm{ppm}$ in the synovium and $1 \mathrm{ppm}$ in all other tissues are assumed for the numerical simulations. The neutron beam direction is normal to the skin and is $8.7 \mathrm{~cm}$ in diameter. Neutron and photon transport in the soft tissues was simulated by the MCNP Monte-Carlo code [20]. The photon fluences computed in the volumes inside the 2.5-cm-diam cylinder intersecting the knee (see Fig. 5.1) are modified by the photon mass attenuation coefficients (see Fig. 2.5 and appendix B.3) of the International Commission on Radiation Units and Measurements report $46[14]$ to compute the photon doses $\mathrm{D}_{\gamma}$. Similarly, the neutron fluences are modified by the fluence-to-KERMA conversion factors (see Fig. 2.4 and appendix B.1) [14] to compute the neutron doses. All reactions happening at neutron energies less than $0.5 \mathrm{eV}$ 


\begin{tabular}{|l||c|c|c|c|}
\hline Element & articular cartilage & bone & joint fluid & tissue \\
\hline \hline Hydrogen & 9.60 & 3.40 & 11.10 & 10.00 \\
\hline Carbon & 9.90 & 15.50 & - & 14.90 \\
\hline Nitrogen & 2.20 & 4.20 & - & 3.50 \\
\hline Oxygen & 74.40 & 43.50 & 88.90 & 71.60 \\
\hline Magnesium & 0.50 & - & - & - \\
\hline Phosphorous & - & 0.20 & - & - \\
\hline Sulfur & 2.20 & 10.30 & - & - \\
\hline Calcium & 0.09 & 22.50 & - & - \\
\hline Chlorine & 0.03 & - & - & - \\
\hline \hline Density $\left[\mathrm{g} / \mathrm{cm}^{3}\right]$ & 1.10 & 1.92 & 1.00 & 1.00 \\
\hline
\end{tabular}

Table 5.1: Density and elemental composition of bone and soft tissue in weight percentages.

(mostly nitrogen absorption reactions) constitute the thermal neutron dose $\mathrm{D}_{t h}$, while all reactions above $0.5 \mathrm{eV}$ (mostly proton recoil reactions) contribute to the fast neutron dose $\mathrm{D}_{f}$. The dosimetric effect of neutrons will depend on the concentrations of ${ }^{10} \mathrm{~B}$ in both synovium and healthy tissues. To estimate the ${ }^{10} \mathrm{~B}(n, \alpha)^{7} \mathrm{Li}$ contribution $\mathrm{D}_{B}$ to the dose, the neutron fluence was modified by ${ }^{10} \mathrm{~B}$ fluence-to-KERMA conversion factors (see Fig. 2.4 and appendix B.2), listed in Caswell et al. [16], and then multiplied by either a factor of 1 $\mathrm{ppm}$ (to represent the $1 \mu \mathrm{g} / \mathrm{g}$ of ${ }^{10} \mathrm{~B}$ in healthy tissues) or a factor of $1000 \mathrm{ppm}$ (to represent the $1000 \mu \mathrm{g} / \mathrm{g}$ of ${ }^{10} \mathrm{~B}$ in synovium). The total absorbed tissue doses are obtained by combining the individual dose components weighted by their RBE factors, using the following equation:

$$
D_{\text {total }}=R B E_{B} \cdot D_{B}+R B E_{t h} \cdot D_{t h}+R B E_{f} \cdot D_{f}+R B E_{\gamma} \cdot D_{\gamma} \quad[\text { Gy-equivalent } / n]
$$

where the following assumptions are made: ${ }^{10} \mathrm{~B}$ reaction products $R B E_{B}$ is 4.0 , thermal neutron reaction $R B E_{t h}$ is 3.8 , fast neutron reaction $R B E_{f}$ is $3.8, R B E_{\gamma}$ is 1.0 . The $\mathrm{RBE}$ values and ${ }^{10} \mathrm{~B}$ concentrations were taken directly from values used in Ref. [11].

\subsection{Neutron beam dose-response}

To predict the dose-response to any neutron spectrum, a large number of simulations were performed with monoenergetic neutron beams. Each simulation consisted of a 8.7-cm-diam neutron beam - which corresponds to the knee diameter - located at a distance of $1.65 \mathrm{~cm}$ from the surface of the knee and centered on the axis of the cylinder 
where the absorbed doses are computed. The neutron beam energies ranged from $0.01 \mathrm{eV}$ to $14 \mathrm{MeV}$, with four energies equilogarithmically spaced per decade. This energy range covers cold, thermal, epithermal, and finally the fast neutrons produced by the D-D and D-T reactions. For each simulation, the dose-response for each reaction in Eq. 5.7 was determined along the centerline of the beam through the knee. A large database was constructed from which dose as a function of the energy, position, reaction, and RBE can be determined for any monodirectional neutron beam of diameter $8.7 \mathrm{~cm}$ incident on the knee.

The only necessary input is the neutron and photon energy distributions of the beam. The dose-response to any neutron and photon spectra can be computed instantaneously using this database, since it does not require the Monte-Carlo simulation of radiation transport in the knee. A similar database can be constructed to evaluate the dose-response to any photon beam incident on the knee.

\subsubsection{Analysis of the dose-response databases}

A previous study by Binello et al. [5] showed that low-energy neutrons in the range from thermal energies to $1 \mathrm{keV}$ provide the highest therapeutic ratios. This study was carried out for isotropic neutron beams. One could expect the neutron angular distribution to be mainly isotropic after heavy moderation by low-Z materials, which is required to slow down the high-energy neutrons produced by most neutron sources to the desired energy range. However, the presence of the collimator reduces the number of neutrons directly in line of sight with the knee to a small fraction within the solid angle formed by the exit window of the collimator, see Fig. 5.2. This results in a neutron beam that has a forwardpeaked angular distribution. To illustrate the effect of the collimator on the directionality of the neutron beam, we consider the following example where neutrons from the D-D reaction are thermalized by a 50-cm-thick, 35-cm-diam $\mathrm{D}_{2} \mathrm{O}$ moderator (see Fig. 5.2), surrounded by a 10-cm-thick lead reflector, surrounded itself by a 10-cm-thick lithiated polyethylene layer. The back-reflector is a 10-cm-thick lead layer and is followed by a 10-cm-thick lithiated polyethylene layer. The delimiter between the BSA and the knee is a single 11-cm-thick lithiated polyethylene layer. The collimator is cylindrical, $8.7 \mathrm{~cm}$ in diameter, and not lined by any material. The current-to-flux ratio, which measures beam directionality, is equal to 0.5 for a neutron beam isotropic in $2 \pi$ in the forward direction (referred to as isotropic hereafter). It is equal to 1.0 for a monodirectional neutron beam. In our case, the ratio 


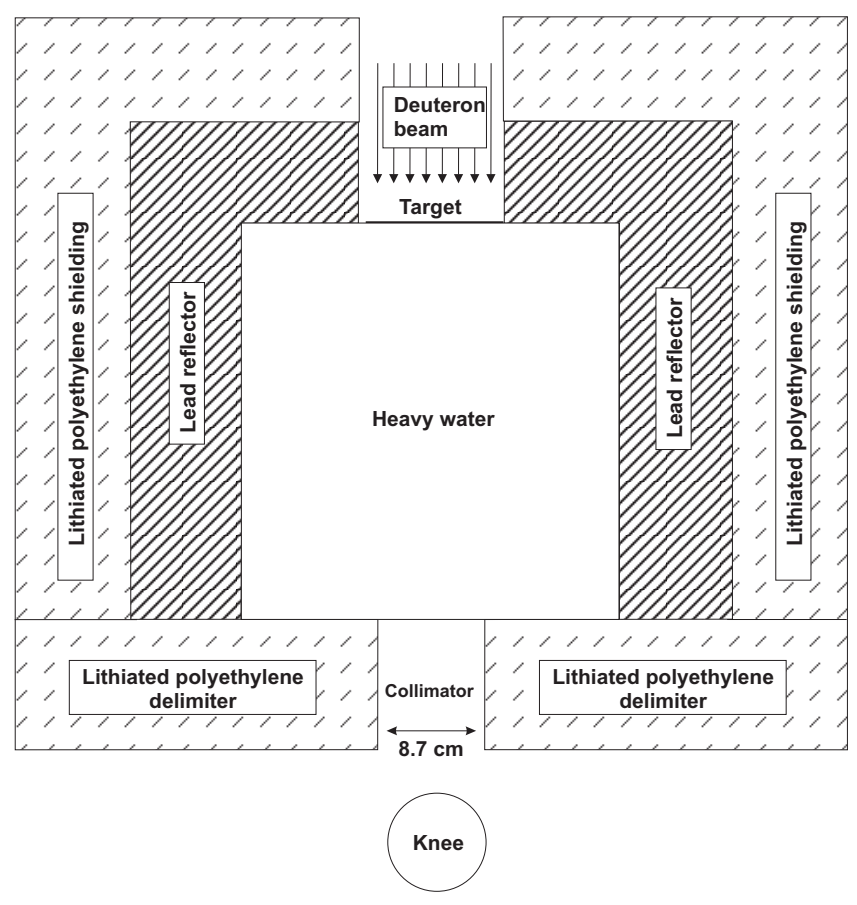

Figure 5.2: Sample beam-shaping assembly (BSA).

is equal to 0.55 before the collimator and 0.90 at the exit window of the collimator. This number reveals the strong directionality of the beam. Since a general neutron beam is partially isotropic and monodirectional, it is interesting to see whether the dose-responses for isotropic and monodirectional neutron beams are similar.

The therapeutic ratios as a function of the neutron energy are shown for both cases in Fig. 5.3. The ratio of the synovium absorbed dose to the average bone absorbed dose decreases as the neutron energy increases for both beam cases. Monodirectional neutron beams are more penetrating than isotropic ones, and their synovium/bone ratios, are, therefore $20 \%$ lower. The ratio of the synovium absorbed dose to maximum front skin absorbed dose increases from thermal energies to $1 \mathrm{eV}$, is approximately constant between $1 \mathrm{eV}$ and $0.5 \mathrm{keV}$, and decreases rapidly down to 0 at about $100 \mathrm{keV}$. This ratio is slightly higher for monodirectional neutron beams than for isotropic ones. To satisfy the 8-Gy-equivalent dose limit on the skin dose, this ratio has to be higher than $100 / 8=12.5$. This condition is satisfied for all neutrons of energy lower than approximately $10 \mathrm{keV}$. To maximize the synovium/bone therapeutic ratio under the skin dose limit constraint, thermal neutron beams are optimal. 


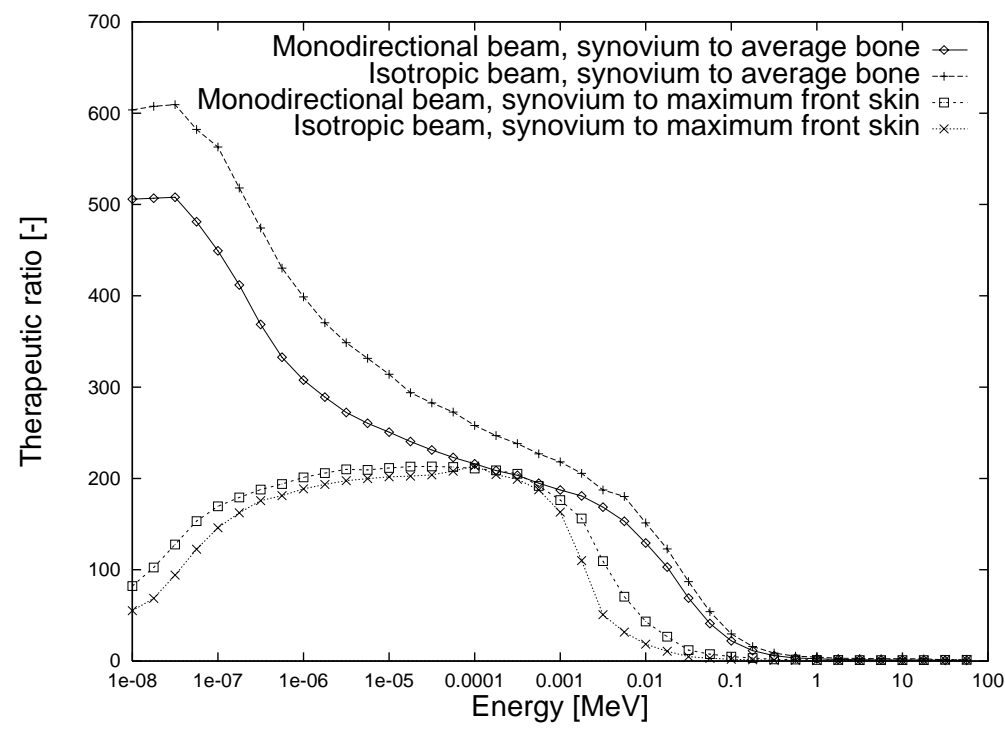

Figure 5.3: Therapeutic ratios as a function of the neutron beam energy for isotropic and monodirectional neutron beams.

The dose rates per unit neutron current at the neutron source for three different positions in the knee are shown in Fig. 5.4. The dose rates to the skin and bone layers

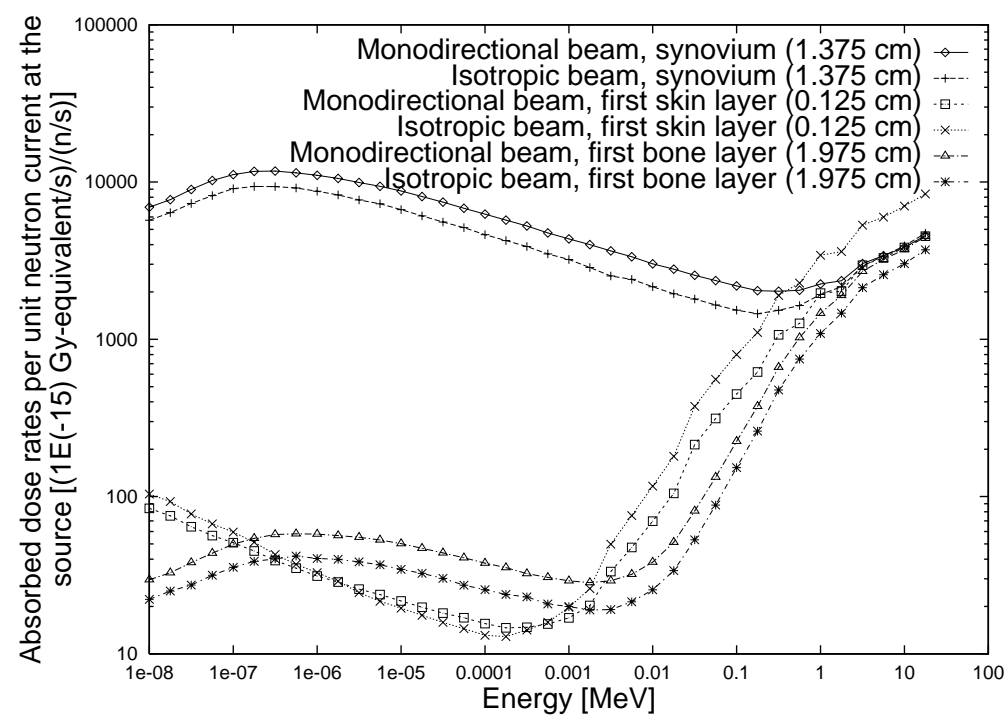

Figure 5.4: Absorbed dose rates per unit neutron current at the neutron source as a function of the neutron beam energy for isotropic and monodirectional neutron beams.

indicated in the graph increase rapidly for energies above $1 \mathrm{keV}$ and $10 \mathrm{keV}$, respectively. For instance, one 14.1-MeV neutron contributes as much to the skin layer dose as 500 neutrons of 
energy $0.1 \mathrm{keV}$ in the isotropic neutron beam case. Moreover, these fast neutrons contribute less to the synovium absorbed dose than neutrons of energy $0.1 \mathrm{keV}$. For this reason and from the therapeutic ratios shown in Fig. 5.3, neutrons in the energy range 5 to $10 \mathrm{keV}$ and higher are therapeutically undesirable. Fast neutron (14-MeV) currents at the entrance of the knee must be at least three orders of magnitude lower than the desired thermal neutron currents not to become the main contributors to the skin dose and therefore decrease the therapeutic ratios. Similar calculations can be performed for all neutron energies. One can then deduce how much each fast component of the neutron current must be decreased to obtain reasonably good therapeutic ratios. An ideal neutron spectrum can be determined based on such an analysis.

Neutrons with energy $0.2 \mathrm{eV}$ maximize the synovium dose per neutron emitted by the source. Each neutron emitted by the source delivers $\sim 10^{-11}$ Gy-equivalent to the synovium. The number of neutrons required to reach the $100-\mathrm{Gy}$-equivalent dose in the synovium is $10^{13}$. Dividing by the area of the beam, this corresponds to a neutron fluence of $1.7 \times 10^{11} \mathrm{n} / \mathrm{cm}^{2}$, or a neutron flux of $2.8 \times 10^{8} \mathrm{n} / \mathrm{cm}^{2} / \mathrm{s}$ for a 10 -min treatment time.

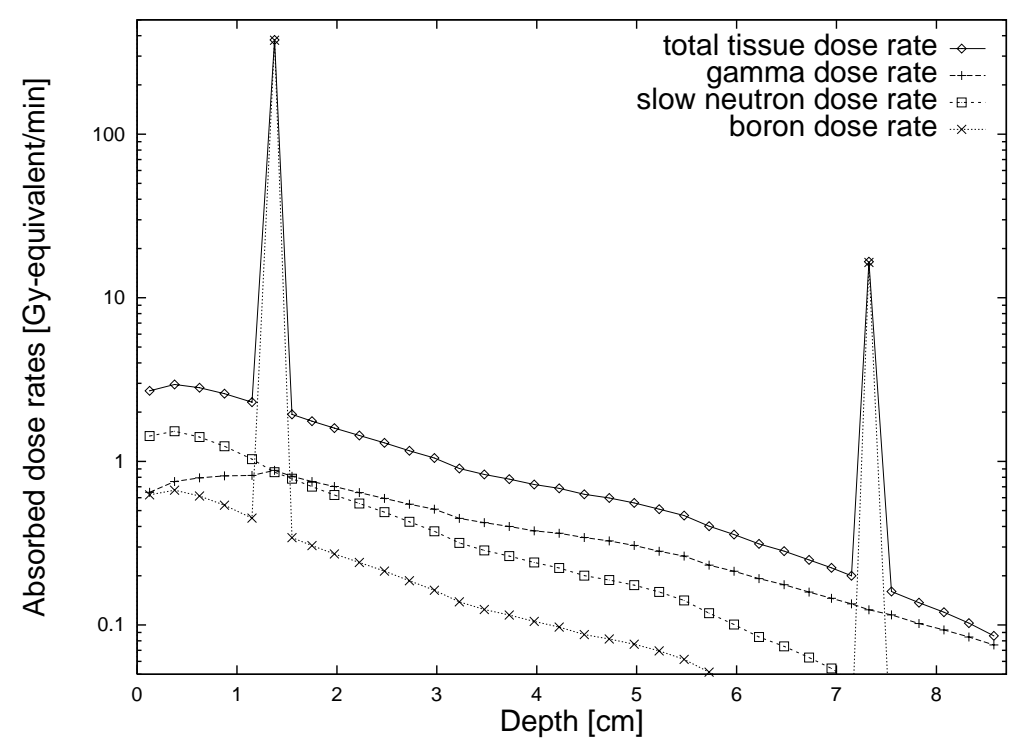

Figure 5.5: Absorbed dose rates versus depth for a 8.7-cm-diam monodirectional neutron beam of energy $0.031 \mathrm{eV}$. A neutron source strength of $7 \times 10^{11} \mathrm{n} / \mathrm{s}$ was used.

Figure 5.5 shows the dose distribution in the knee corresponding to a monodirectional neutron beam of energy $0.031 \mathrm{eV}$. Since only neutrons are irradiating the patient's knee in this simulation study, the gamma dose is strictly induced by interactions of the 
neutrons with the different tissues in the knee. This induced gamma dose (mainly due to the interactions of neutrons with hydrogen) represents about $20 \%$ of the total tissue dose at the surface of the skin and $50 \%$ of the total tissue dose in the center of the knee.

\subsubsection{Use of dose-response database for the design of BSAs}

These dose-response databases can be used to accelerate the design of BSAs for BNCS. MCNP is used to simulate the neutron and photon transport through the BSA. Given a neutron source strength, the neutron and photon currents and fluxes are calculated across the $8.7-\mathrm{cm}$-diam exit window at the end of the BSA, see Fig. 5.2. The dose-response database corresponding to the monodirectional neutron beam is then used to convert the neutron current passing through the exit window of the delimiter into a dose rate distribution in the knee. Since we are using the dose-response database corresponding to an 8.7-cm-diam monodirectional neutron beam, this conversion gives the dose rate distribution in the knee that results from an 8.7 -cm-diam monodirectional neutron beam which has the same neutron current at the exit window of the delimiter as the one computed by MCNP. We will refer to it as the monodirectional neutron beam dose-response. The same procedure can be followed to compute the isotropic neutron beam dose-response using the dose-response database corresponding to the isotropic neutron beam instead of the one corresponding to the monodirectional neutron beam. To compute the dose-response of the neutron beam coming out of the delimiter (referred to as the real neutron beam), which is neither monodirectional nor isotropic, we will combine the monodirectional with the isotropic neutron beam dose-responses in such a way that the combination is a good estimation of the actual dose distribution in the knee.

The procedure is based on the fact that any neutron beam can be approximated by the weighted sum of a monodirectional neutron beam and an isotropic neutron beam. Different approximations are possible; the one used in this study is based on the equality of the neutron beam directionalities. The directionality $\alpha$ of the real neutron beam - or any neutron beam - is the ratio of the neutron current density at the exit window of the delimiter to the neutron flux density at the same location, i.e., $J_{+} / \phi$. To obtain the same directionality $\alpha$ from a combination of a monodirectional neutron beam and an isotropic neutron beam, one has to combine a fraction $2 \cdot \alpha-1$ of the monodirectional neutron beam with a fraction $2 \cdot(1-\alpha)$ of the isotropic neutron beam. Indeed, by integrating the weighted 
sum of the angular neutron densities $\mathrm{n}_{\text {monodirectional }}(\theta, \varphi)=\frac{\delta(\varphi-\pi / 2)}{2 \pi}$ and $\mathrm{n}_{\text {isotropic }}(\theta, \varphi)=\frac{1}{2 \pi}$ corresponding to the two beam types over all solid angles corresponding to positive currents, one obtains a neutron current

$$
J_{+}=\int_{\theta=0}^{\theta=2 \pi} d \theta \int_{\varphi=0}^{\varphi=\pi / 2} d \varphi\left[(2 \cdot \alpha-1) \cdot \frac{\delta(\varphi-\pi / 2)}{2 \pi}+2 \cdot(1-\alpha) \cdot \frac{1}{2 \pi} \cos (\varphi) \sin (\varphi)\right]=\alpha,
$$

and a neutron flux

$$
\phi=\int_{\theta=0}^{\theta=2 \pi} d \theta \int_{\varphi=0}^{\varphi=\pi / 2} d \varphi\left[(2 \cdot \alpha-1) \cdot \frac{\delta(\varphi-\pi / 2)}{2 \pi}+2 \cdot(1-\alpha) \cdot \frac{1}{2 \pi} \cos (\varphi)\right]=1,
$$

that result in the same neutron beam directionality $J_{+} / \phi=\alpha$ as the real neutron beam. Note that the two angular neutron densities $\mathrm{n}_{\text {monodirectional }}(\theta, \varphi)$ and $\mathrm{n}_{\text {isotropic }}(\theta, \varphi)$ are normalized in such a way that their integral over all solid angles corresponding to positive currents are equal to 1.

The monodirectional and isotropic neutron beam dose-responses will be weighted the same way to obtain an approximation of the dose distribution in the knee for the real neutron beam. The same procedure can be used to convert the photon current at the exit window of the delimiter into a dose distribution corresponding to the photons passing through the exit window of the delimiter. Adding up the two dose distributions, we can compute the dose distribution in the knee due to the neutrons and photons going through the exit window.

This method will be referred to as the "dose-response database method". It will be compared with the "full simulation method", where the neutron and photon transport simulation from the neutron source to the knee is performed entirely by the MCNP code. For both methods, different variance-reduction techniques such as geometry splitting with Russian roulette, weight windows, and angle biasing with DXTRAN are used to decrease the simulation time. The dose-response database method is computationally less time consuming than the full-simulation method, it is approximately 10 times faster. The drawback of this method is that it does not account for the particles transmitted through the delimiter. Only the particles crossing the exit window are used to compute the dose-response. For this reason, the delimiter has to be designed using the full-simulation method, with the goal of reducing to negligible values the radiation due to leakage through the delimiter. Once the delimiter has been shown to efficiently attenuate radiation leakage through the delimiter using the full-simulation method, the dose-response database method can be used more extensively for the design of neutron beams. 


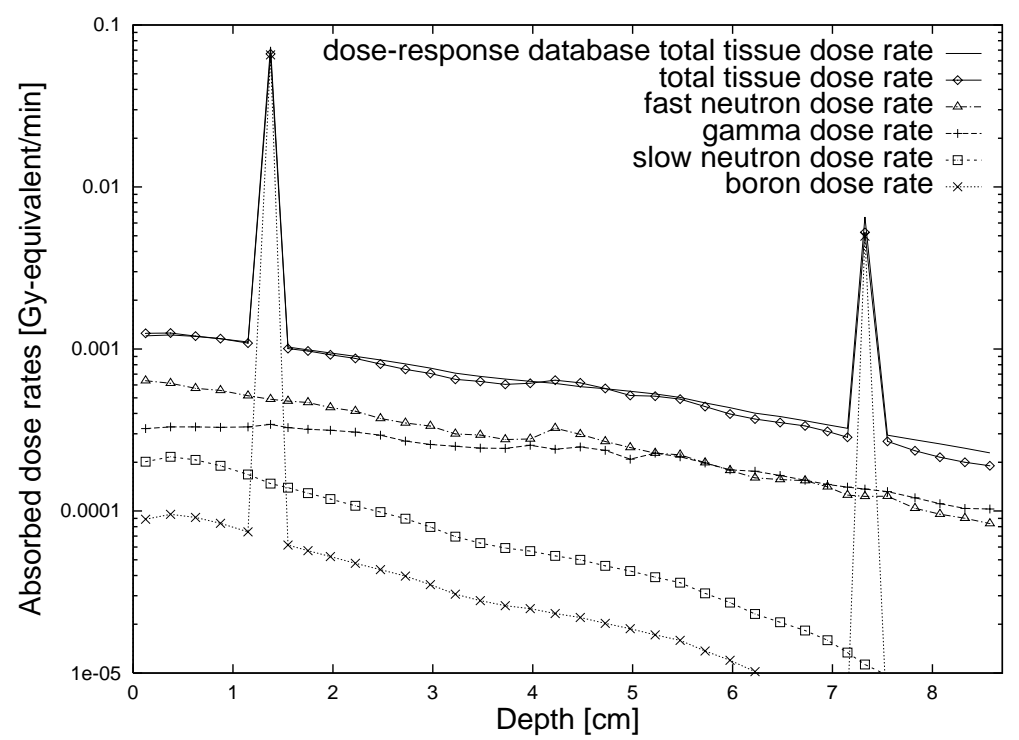

Figure 5.6: Comparison of the dose-response database method with the full simulation method for the computation of the absorbed dose rates. A D-D neutron source of strength $7 \times 10^{11} \mathrm{n} / \mathrm{s}$ was used.

Figure 5.6 compares the dose distributions computed using the dose-response database and full-simulation methods. The D-D neutron source and BSA used for the comparison are the ones described in Secs. 6.1 and 5.4.1. This moderator, composed primarily of heavy water, was chosen because of its high moderating ratio and low photon production, which lead to high therapeutic ratios. For the sake of clarity, only the total tissue absorbed dose is shown for the computations with the dose-response database method. As we will see in Sec. 6.3, the delimiter used for this simulation is very efficient for the attenuation of neutron fluxes. Leakage of photons through the delimiter is not of primary concern, because of the low level of photon fluxes at the end of the moderation. Even without the delimiter, photons coming from this moderator contribute less than $5 \%$ of the total dose in the knee. We observe in Fig. 5.6 that the two methods give significantly identical total absorbed doses for most depths in the knee. More comparisons between dose distributions computed with the full simulation and the dose-response database methods are included in Secs. 6.5 and 6.6 to validate the dose-response database method. In the case of the dose distribution corresponding to the pure thermal neutron beam shown in Fig. 5.5 - which is close to an ideal neutron beam - , the main contributor to the total dose is the dose corresponding to the thermal neutrons. In Fig. 5.6, the total absorbed dose is mostly 
due to the fast neutron component of the neutron beam through proton recoil reactions.

\subsection{Summary of results}

Using Monte Carlo simulations to model neutron beam interaction with a phantom knee, guidelines were developed for determining the optimal neutron beam energy for delivering a dose to the target area, the synovium. Two figures-of-merit are used to measure the beam quality: the ratio of the synovium absorbed dose to the skin absorbed dose and the ratio of the synovium absorbed dose to the bone absorbed dose. It was found that (a) thermal neutron beams are optimal for BNCS treatment, (b) similar absorbed dose rates and therapeutic ratios are obtained with monodirectional and isotropic neutron beams. The thermal neutron flux required to deliver 100 Gy-equivalent to the synovium in $10 \mathrm{~min}$ is approximately $2.8 \times 10^{8} \mathrm{n} / \mathrm{cm}^{2} / \mathrm{s}$. The calculated dose rates and ratios depend on the ${ }^{10} \mathrm{~B}$ concentrations in the synovium and healthy tissues. $\mathrm{A}^{10} \mathrm{~B}$ concentration of $1000 \mathrm{ppm}$ was assumed in the synovium, while $1 \mathrm{ppm}$ was assumed in the healthy tissues and bone.

Computation of the dose distribution in the knee requires the simulation of the neutron and photon transport from the neutron source to the knee phantom through the complex BSA. A method - namely the dose-response database method - was developed to predict the absorbed dose distribution in the knee based on any neutron and photon spectra incident on the knee. This method enables one to reduce the neutron and photon transport simulation time by a factor 10 by modeling the transport in the moderator only. Good agreement was observed between dose distributions computed using this method and dose distributions computed using simulation of the entire model, i.e., moderator and knee phantom. 


\section{Chapter 6}

\section{Design of BSAs for BNCS for the D-D and D-T neutron sources}

A previous study [39] showed that the fusion reactions D-D and D-T did not fulfill the requirements for BNCS, i.e., high therapeutic ratios and short treatment times. Based on the experience acquired on moderation of high-energy neutrons in the context of BNCT, it was believed that the BSAs used in this study were not optimal. Therefore, the use of D-D and D-T neutrons for BNCS was reinvestigated in light of our work for BNCT.

Neutrons from both fusion reactions have to be moderated down to thermal energies. The following sections focus on designing BSAs to shape the most suitable neutron beam. The two first sections describe the neutron source and the BSA, the third one concentrates on the design of the delimiter, the fourth one explains the methodology used to optimize the BSA, while the fifth and sixth ones focus on the moderation of D-D and D-T neutrons.

\subsection{Neutron source characterization}

In our numerical simulations, the neutron source is identical to the one described previously in the part on BNCT. We briefly recall its main characteristics. Neutrons are emitted isotropically and monoenergetically across a 5-cm-diam flat circular surface. The source is distributed uniformly over the surface of the target, which is composed of a 10$\mu$ m-thick titanium layer on a 1-mm-thick copper substrate water-cooled on the back by 3 -mm-deep water channels machined in a 5-mm-thick stainless-steel plate. The spread in 
the D-D and D-T neutron energies due to deuteron straggling in the target is not considered in this study. As mentioned in Sec. 4.2, angular isotropy in the lab system is an adequate approximation for D-T neutrons and deuteron beams of low energy. For D-D, the angular distribution in the center-of-mass system is anisotropic. A better modeling of the source accounting for the angular distribution would be required but this is beyond the scope of this work.

\subsection{BSA description}

Source neutrons enter a cylindrical BSA with the monoenergetic neutron distribution corresponding to D-D or D-T. They travel through the BSA composed of several layers of different materials until they reach the other side where the knee is located, see Fig. 6.1. Thick lead and lithiated polyethylene [29] layers separate the BSA from the knee in order

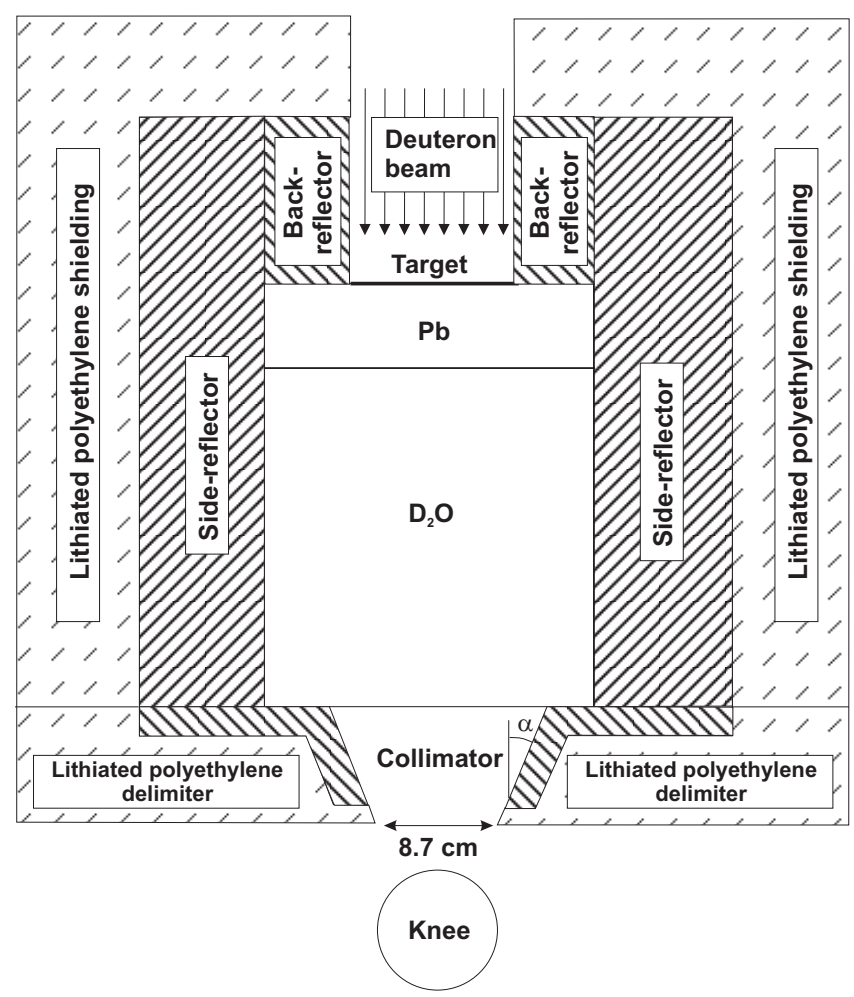

Figure 6.1: BSA showing the 8.7-cm-diam exit window where the neutron and photon spectra are calculated, referred to as exit window throughout the text. The collimator is the truncated cone of angle of aperture $\alpha$ located between the moderator and the exit window. 
to restrict the photon and neutron fluxes to a $8.7-\mathrm{cm}$-diam window, which corresponds to the diameter of the knee. The role of these layers is to decrease radiation exposure to the organs other than the knee. The collimator is the truncated cone located between the moderator and the exit window. It can be lined by a layer of lead or other material in order to collimate the neutron beam to the exit window.

\subsection{Neutron beam delimiter}

The role of the delimiter (see Fig. 6.1) is to decrease radiation exposure to the organs other than the knee. It has to attenuate both neutron and photon fluxes coming out of the moderator efficiently. Lithiated polyethylene is commonly used to reduce photon fluxes. The light elements $\mathrm{C}$ and $\mathrm{H}$ thermalize the neutrons, while lithium enriched in its isotope ${ }^{6} \mathrm{Li}$ absorbs the thermalized neutrons.

\subsubsection{Delimiter thickness and material}

Using the BSA described in Sec. 5.4.1, several simulations are performed using different lithiated polyethylene delimiter thicknesses. Two quantities are used to compare the performances of the delimiters, they are shown against the delimiter thickness in Fig. 6.2. The first one measures the decrease in neutron current at the exit window due to the presence of the delimiter. It is defined as the ratio of the neutron current across the exit window with the delimiter to the neutron current across the exit window without the delimiter. Note that the exit window is at the end of the delimiter, its position varies thus with the delimiter thickness. For lithiated polyethylene, this ratio decreases as the delimiter increases, while the directionality of the beam (shown in Fig. 6.3) increases. This means that most of the neutrons hitting the walls of the collimator are eventually absorbed in the delimiter. On the contrary, if graphite or lead are used as a delimiter, the ratio first increases to reach a maximum of 1.16 for a thickness of $4 \mathrm{~cm}$ and then slowly decreases, while the directionality of the beam slowly but steadily increases. This small increase can be qualitatively explained. The lead and graphite layers reflect neutrons - that would otherwise be absorbed in the lithiated polyethylene - back to the moderator. These neutrons then contribute to the current at the exit window of the collimator. This leads to the conclusion that the neutron current across the exit window can be increased by about $16 \%$ by inserting a layer of either graphite or lead between the moderator and the delimiter. This layer will 


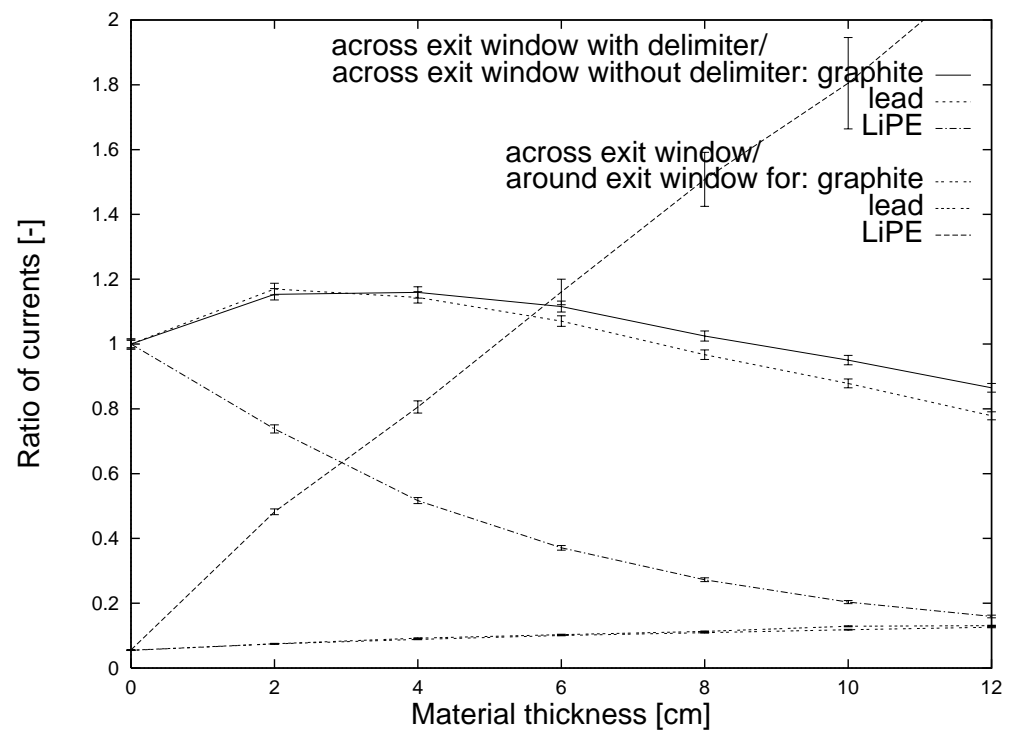

Figure 6.2: Delimiter thickness and material parametric study. Ratios of neutron current across the exit window with the delimiter to neutron current across the exit window without delimiter, and neutron current across the exit window to neutron current around the exit window with the delimiter, as a function of the delimiter thickness.

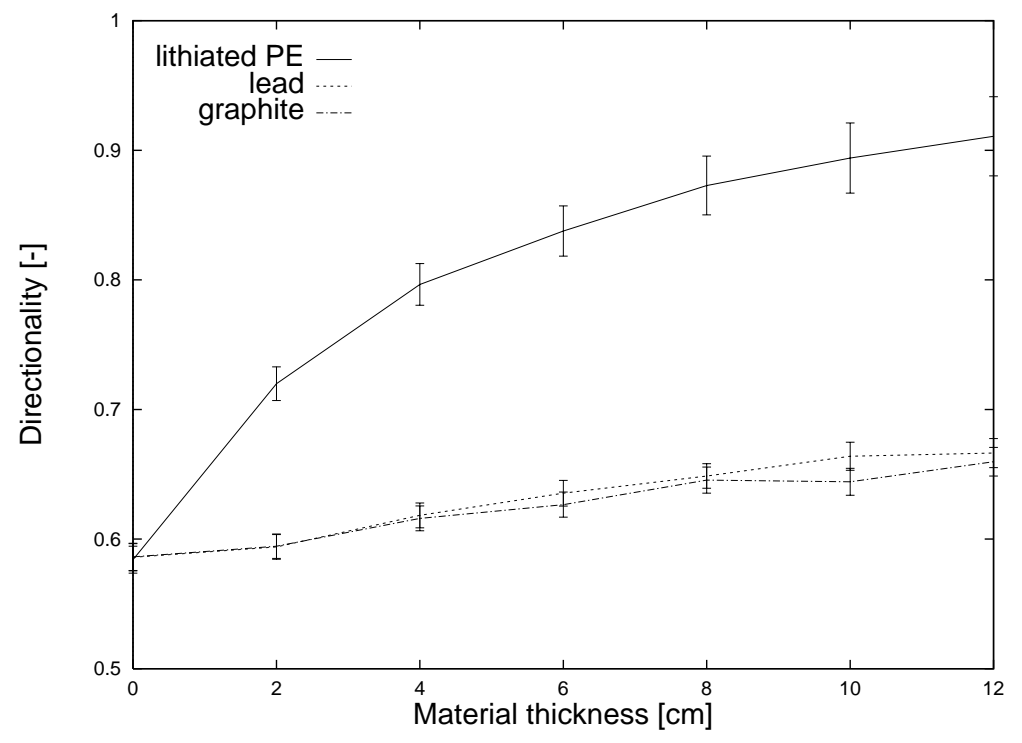

Figure 6.3: Delimiter thickness and material parametric study. Directionality - measured by the ratio of the current $J_{+}$to the flux $\phi$ across the exit window - versus delimiter thickness.

systematically be added to our BSAs. While lead is advantageous for the attenuation of photons, graphite is preferable when photons are not an issue, since the density of graphite 
makes it mechanically much easier to handle.

The second quantity measures the delimiter "attenuation efficiency". It is defined as the ratio of the neutron current across the exit window (of diameter $8.7 \mathrm{~cm}$ ) to the neutron current in the annular area of inner diameter equal to the exit window diameter and outer diameter equal to the reflector outer diameter $(55 \mathrm{~cm})$. Since the ratio of the areas is $\pi \cdot 4.35^{2} / \pi \cdot\left(27.5^{2}-4.35^{2}\right) \simeq 1 / 39$, the ratio of the neutron current density across the exit window to the one outside the exit window can be found by multiplying the neutron attenuation efficiency by 39 .

Figure 6.2 shows that the neutron attenuation efficiency steadily increases for lithiated polyethylene. Lead and graphite are very poor materials in that regard. A 8-cmthick layer of lithiated polyethylene will be used in our BSAs to reduce neutron leakage through the delimiter. This will lead to an average neutron current density 60 times lower outside the exit window than across the exit window.

\subsubsection{Collimator thickness and angle}

Using a 5-cm-thick graphite layer followed by a 8-cm-thick layer of lithiated polyethylene as a delimiter, we now study the influence of the angle $\alpha$ and the thickness of the collimator (as shown in Fig. 6.1). The material used for the collimator is also graphite. A 2-cm-thick layer of lithiated polyethylene (see Fig. 6.1) is left between the collimator and the knee in order to compensate for the lower neutron attenuation of the collimator material. Figure 6.4 shows the ratio of the neutron current across the exit window with the delimiter to the neutron current across the exit window without delimiter for different collimator angles and thicknesses. This ratio, which measures the neutron output gain or loss due to the delimiter, increases steadily with the collimator angle $\alpha$ until it reaches its maximum at an angle of $40^{\circ}$, and then starts decreasing. It also increases with the collimator thickness. A thick collimator of angle $40^{\circ}$ seems to be optimal for neutron collimation. However, Fig. 6.5 shows that the neutron attenuation efficiency decreases steeply with the collimator angle, past a critical angle of $20^{\circ}$.

Thin collimators have lower attenuation efficiencies for small angles, while they have higher ones for large angles. This can qualitatively be explained by the fact that some neutrons are reflected off the walls of the graphite collimator and directed towards the exit window, contributing to the numerator of the ratio, while they are absorbed by the 


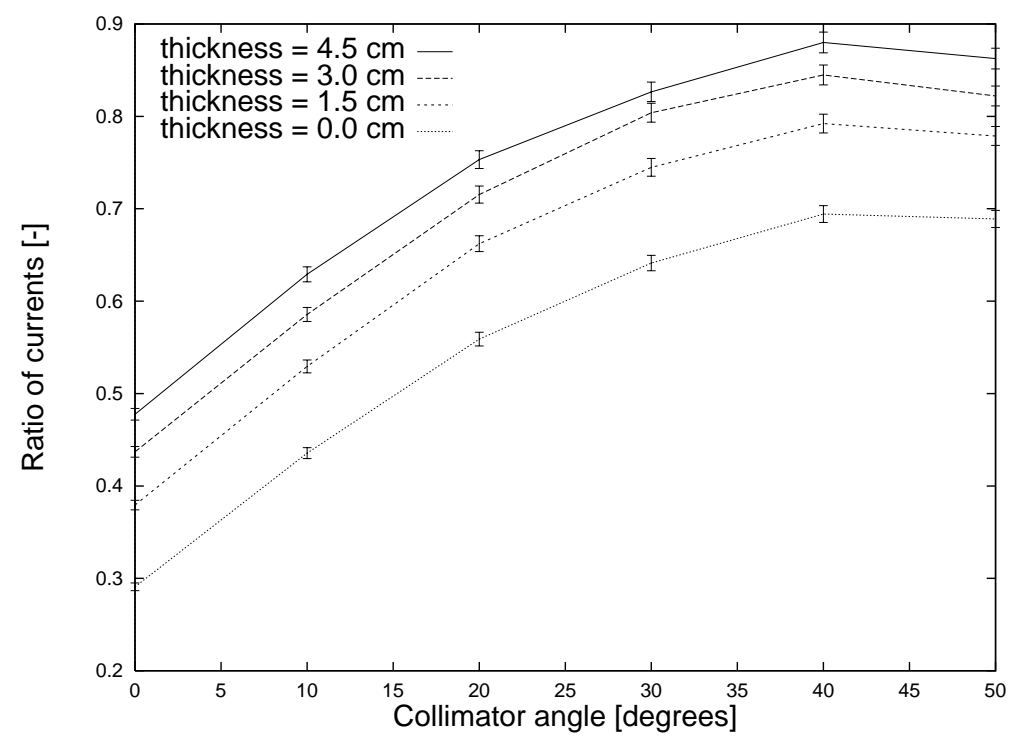

Figure 6.4: Collimator angle and thickness parametric study. Ratio of neutron current across the exit window with the delimiter to neutron current across the exit window without delimiter, as a function of the graphite collimator angle and thickness. The delimiter is composed of a 5-cm-thick graphite layer followed by a 8-cm-thick lithiated polyethylene layer.

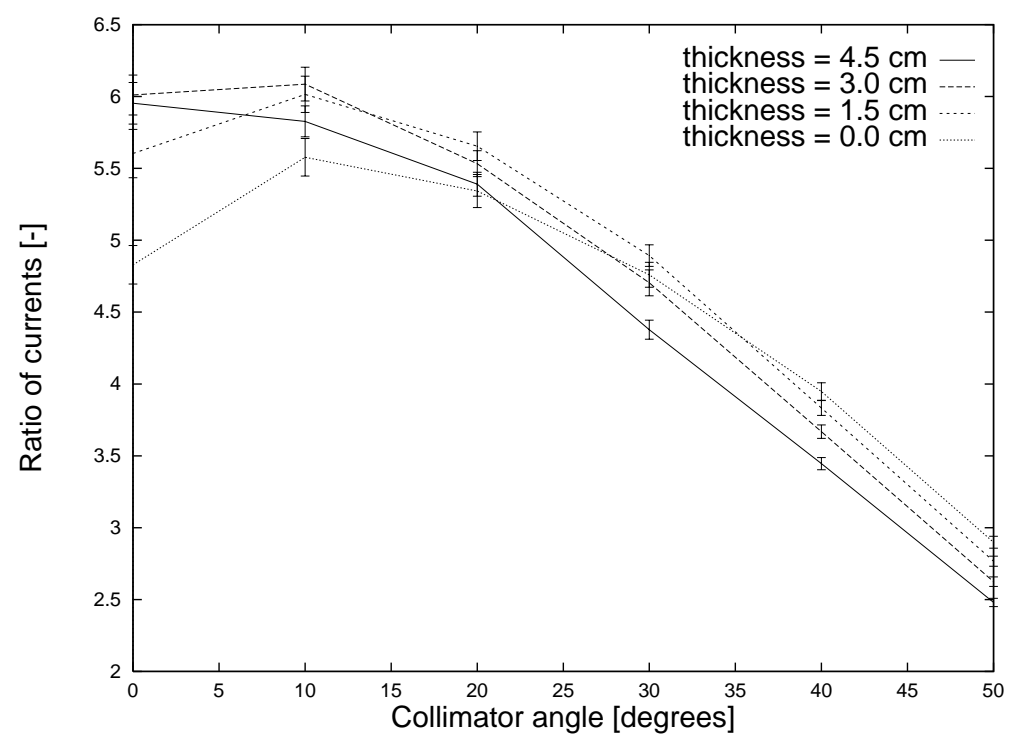

Figure 6.5: Collimator angle and thickness parametric study. Ratio of neutron current across the exit window to neutron current around the exit window with the delimiter, as a function of the graphite collimator angle and thickness. The delimiter is composed of a 5-cm-thick graphite layer followed by a 8 -cm-thick lithiated polyethylene layer. 
delimiter, if no collimator is present. For very thick collimators however (see 4.5-cm-thick case), the higher neutron leakage through the delimiter due to the presence of the thick collimator is not compensated by the increase in neutron current in the exit window and therefore the ratio becomes lower. Considering both Fig. 6.4 and 6.5, one can conclude that $1.5-\mathrm{cm}$-thick and 3.0-cm-thick graphite collimators of angle $20^{\circ}$ are close to optimal. A 3-cm-thick collimator of angle $20^{\circ}$ will be used further in this study, because it gives a slightly higher ratio in Fig. 6.4. The same work has been repeated for lead instead of graphite for the collimator and for the 5-cm-thick layer preceding the 8-cm-thick layer of lithiated polyethylene. The results were very similar and lead could easily substitute for graphite in case photon production in the moderator is of concern.

\subsection{Methodology for the optimization of the BSAs}

The quantities used to measure the quality of the neutron beam coming out of the BSA are those defined in Sec. 5.2 of Chapter 5: the ratio $D_{\text {syn }} / D_{\text {bone }}$ of the absorbed synovium dose to the average absorbed bone dose, the ratio $D_{s y n} / D_{s k}$ of the absorbed synovium dose to the maximum absorbed front skin dose, the absorbed synovium dose $\mathrm{N} \cdot \mathrm{D}_{\text {syn }}$, and the treatment time T. Several parameters can be varied for the optimization, the materials $\mathrm{M}_{i}$ of the BSA, the number I of different materials, the material thicknesses $\mathrm{Th}_{i}$, the BSA diameter $\mathrm{D}$, the side- and back-reflector materials $\mathrm{M}_{\text {side-refl }}$ and $\mathrm{M}_{\text {back-refl }}$, the side- and back-reflector thicknesses $\mathrm{Th}_{\text {side-refl }}, \mathrm{Th}_{\text {back-refl }}$, etc. Even though one could allow these parameters to vary to infinity, one will set limits on their ranges. Because of weight concerns, the BSA diameter $D$ will vary in the range 0 to $100 \mathrm{~cm}$, the side- and backreflector thicknesses $\mathrm{Th}_{\text {side-refl }}$ and $\mathrm{Th}_{\text {back-refl }}$ in the range 0 to $50 \mathrm{~cm}$. The number of materials I in the BSA will be limited to 3 . The reflector materials $\mathrm{M}_{\text {refl }}$, as well as the neutron moderating materials $\mathrm{M}_{i}$ will be chosen based on the work done in Chapter 4 for BNCT. Let $\vec{x}$ be an array containing all the parameters.

$\vec{x}=\left(M_{i}(1 \leq i \leq I) \quad T h_{i}(1 \leq i \leq I) \quad D \quad M_{\text {side-refl }}\right.$ Th $\left.h_{\text {side-refl }} M_{\text {back-refl }} \quad T h_{\text {back-refl }}\right)$.

The optimal BSA will be such that the set of parameters gives the highest therapeutic ratio $D_{\text {syn }} / D_{\text {bone }}$

$$
\frac{D_{\text {syn }}}{D_{\text {bone }}}\left(\vec{x}_{\text {optimal }}\right)=\max \left(\frac{D_{\text {syn }}}{D_{\text {bone }}}(\vec{x})\right)
$$


for all possible $\vec{x}$ under the following constraints:

$$
T\left(\vec{x}_{\text {optimal }}\right) \leq T_{\max }
$$

for the treatment time $\mathrm{T}$,

$$
\frac{D_{\text {syn }}}{D_{s k}}\left(\vec{x}_{\text {optimal }}\right) \geq 12.5
$$

for the therapeutic ratio absorbed synovium dose to absorbed maximum front skin dose, and

$$
N \cdot D_{\text {syn }}\left(\vec{x}_{\text {optimal }}\right)=100 \quad \text { Gy-equivalent }
$$

for the absorbed synovium dose. The number of source neutrons $\mathrm{N}$ is determined using Eq. 6.5. The treatment time $\mathrm{T}$ is determined using Eq. 5.4. The neutron source strength $\mathrm{S}$ depends on the fusion reaction considered. In this Chapter, we will use $7 \times 10^{11} \mathrm{n} / \mathrm{s}$ for D-D (see Sec. 6.5) and $10^{14} \mathrm{n} / \mathrm{s}$ for D-T (see Sec. 6.6). The maximum permissible treatment time $\mathrm{T}_{\max }$ is set to $10 \mathrm{~min}$.

With 3 materials in the BSA, the number of independent variables is 11 . The ratio $D_{\text {syn }} / D_{\text {bone }}(\vec{x})$ is a 11-dimensional hypersurface imbedded in a 12-dimensional hyperspace. Because of the complexity of this optimization problem, one has to make some assumptions. Let $\vec{x}_{s u b}$ be the set of parameters $\vec{x}$ minus its $\mathrm{M}_{\text {side-refl }}$ and $\mathrm{Th}_{\text {side-refl }}$ components.

$$
\vec{x}_{s u b}=\left(M_{i}(1 \leq i \leq 3) \quad T h_{i}(1 \leq i \leq 3) \quad D \quad M_{b a c k-r e f l} \quad T h_{b a c k-r e f l}\right)
$$

One will assume that if the side-reflector material $\mathrm{M}_{\text {side-refl }}$ and thickness $\mathrm{Th}_{\text {side-refl }}$ are optimal for one subset $\vec{x}_{s u b}$ of parameters, they will be optimal for all subsets $\vec{x}_{s u b}$ of parameters. The same assumption will be made for the back-reflector material $\mathrm{M}_{b a c k-r e f l}$ and thickness Thback-refl, as well as for the BSA diameter D. With these assumptions, one can optimize (a) the side-reflector material and thickness, (b) the back-reflector material and thickness, and (c) the BSA diameter, without optimizing simultaneously the other parameters. Once the ratio $D_{\text {syn }} / D_{\text {bone }}(\vec{x})$ is maximized in terms of these parameters, the optimization problem is reduced to finding the maximum of a 6 -dimensional hypersurface in a 7-dimensional hyperspace. Furthermore, in light of our work for BNCT, we will limit the number of moderating materials to just a few: heavy water for D-D neutrons, lead, lithium fluoride, and heavy water (in this order) for D-T neutrons. The optimization problem reduces then to finding the maximum of a line in a 2-dimensional plane for D-D, and the maximum of a 3-dimensional hypersurface imbedded in a 4-dimensional hyperspace for $\mathrm{D}$ - $\mathrm{T}$. 
The optimization must be performed with the constraints mentioned above. More on the optimization method can be found in Sec. 4.5.

\subsection{Moderation of D-D neutrons}

First, a generic moderator is used to analyze the impact of the reflector material, the reflector thickness and the BSA diameter. Then, different combinations of materials are considered, with the goal of producing an intense thermal neutron beam with the fast neutron component reduced to a minimum level.

It is worth briefly examining the neutron cross sections of heavy water. The data are taken from the MCNP [20] cross section library. As shown in Fig. 6.6, heavy water has a high elastic scattering cross section and a very low absorption cross section at low energies.

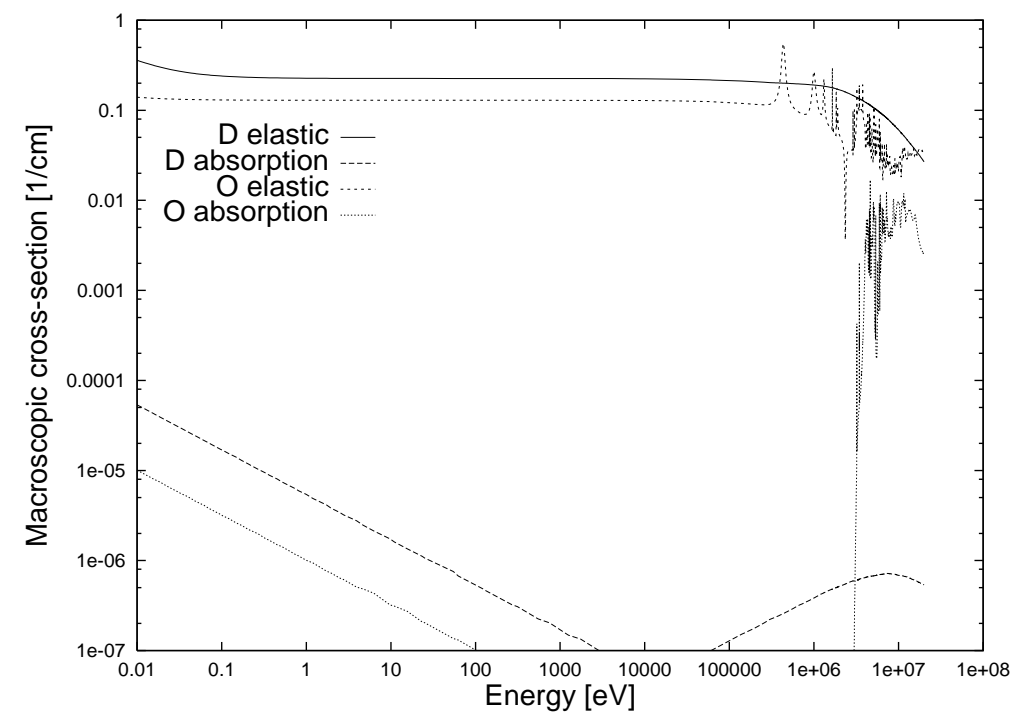

Figure 6.6: Macroscopic cross sections of heavy water.

Its moderating ratio is very high in that range. However, the elastic scattering cross section of deuterium decreases at energies higher than $2 \mathrm{MeV}$. It is reduced by approximately a factor 4 between $2.4 \mathrm{MeV}$ and $14 \mathrm{MeV}$. Moreover, the absorption cross section of oxygen increases steeply at $3 \mathrm{MeV}$. Therefore, the moderating ratio of heavy water for D-T source neutrons will be much lower than for D-D source neutrons.

The generic moderator used for the BSA optimization in Secs. 6.5.1 through 6.5.4 is identical to the one described in Sec. 5.4.1. The delimiter between the heavy water 
moderator and the knee, as well as the collimator, are different. As described in Sec. 6.3, the delimiter is composed of a 5-cm-thick graphite layer followed by a 8-cm-thick lithiated polyethylene layer. The collimator is lined with a 3-cm-thick graphite layer at an angle of $20^{\circ}$. A 2-cm-thick layer of lithiated polyethylene (see Fig. 6.1) is left between the collimator and the knee in order to compensate for the lower neutron attenuation of the collimator material. A D-D neutron source strength of $7 \times 10^{11} \mathrm{n} / \mathrm{s}$ is assumed at the target. This strength corresponds to a neutron generator in which a 1.5-A mainly monatomic deuteron beam of energy $150 \mathrm{keV}$ hits a large area $T i D_{1.6}$ target, see Sec. 7.2.2. Unless specified otherwise, this delimiter, collimator and neutron source strength will be used further in the BSA optimization for D-D neutrons.

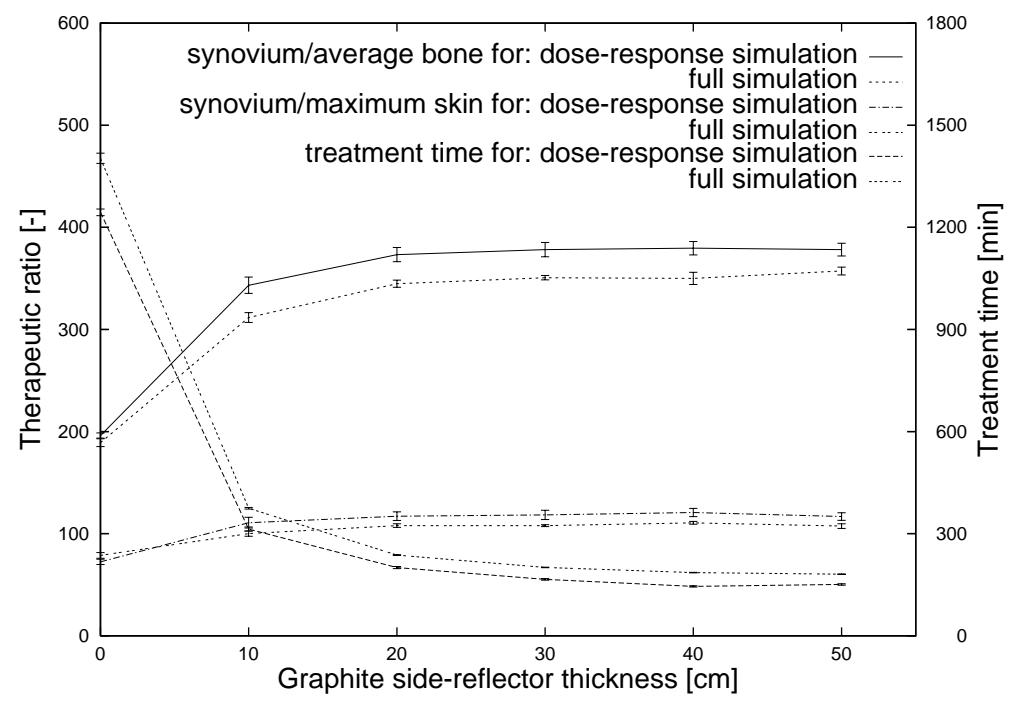

Figure 6.7: Comparison of treatment times and therapeutic ratios obtained with the doseresponse database and the full simulation methods.

Figure 6.7 shows a comparison between the treatment times and therapeutic ratios obtained using the full simulation and the dose-response database methods for different graphite side-reflector thicknesses. The comparison shows an overall good agreement between the two simulation methods. The agreement between the trendlines shows that the dose-response database method can be used for the optimization of the BSA, since the maxima and minima occur for the same side-reflector thicknesses. The good agreement between the values confirms the fact that most particles reaching the knee are transmitted through the exit window of the BSA, as opposed to leaking through the delimiter. 


\subsubsection{Side-reflector design}

The side-reflector material and thickness are varied to see the impact of these two parameters on the treatment time and therapeutic ratios. Three materials are used for the simulations: graphite, lead and aluminium oxide $\mathrm{Al}_{2} \mathrm{O}_{3}$. The side-reflector thicknesses range from $0 \mathrm{~cm}$ to $50 \mathrm{~cm}$. The $\mathrm{BSA}$ is otherwise the same as the one described in Sec. 6.5. The results of this simulation study are presented in Figs. 6.8 and 6.9. Graphite gives the

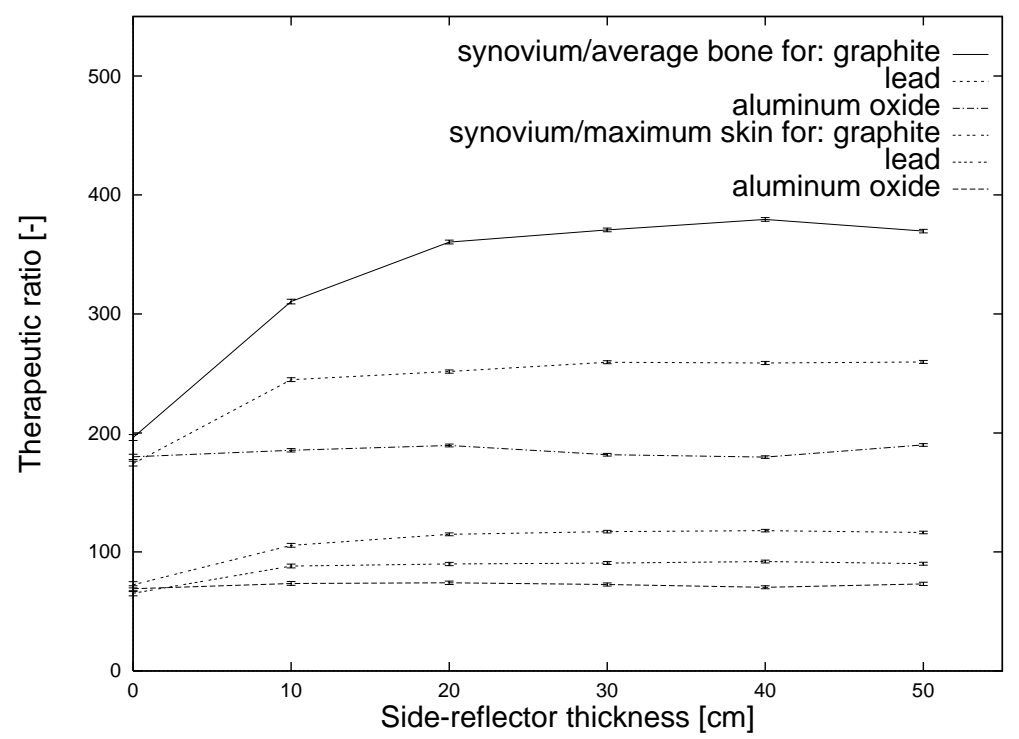

Figure 6.8: Side-reflector material and thickness parametric study. Therapeutic ratios as a function of the side-reflector material and thickness.

highest therapeutic ratios for all side-reflector thicknesses, while aluminum oxide gives the lowest. A more detailed analysis has been carried out to explain these effects. Aluminum oxide generates more photons than graphite and lead when bombarded by neutrons. The excessive photon dose in the knee results in lower therapeutic ratios. Concerning lead, it has a lower moderating power than graphite. Therefore, more fast neutrons are reflected from the lead than from the graphite reflector. This higher fast neutron component in the neutron beam decreases by itself and by photon induction in the tissues the therapeutic ratios. The therapeutic ratios increase asymptotically with the reflector thickness, to reach plateaux at about $30 \mathrm{~cm}$. The treatment time decreases very rapidly with reflector thicknesses up to $30 \mathrm{~cm}$. Graphite has the shortest treatment times for all side-reflector thicknesses. As a conclusion, a 30-cm-thick graphite side-reflector will be used for the simulations in Secs. 6.5.2 through 6.5.4. 


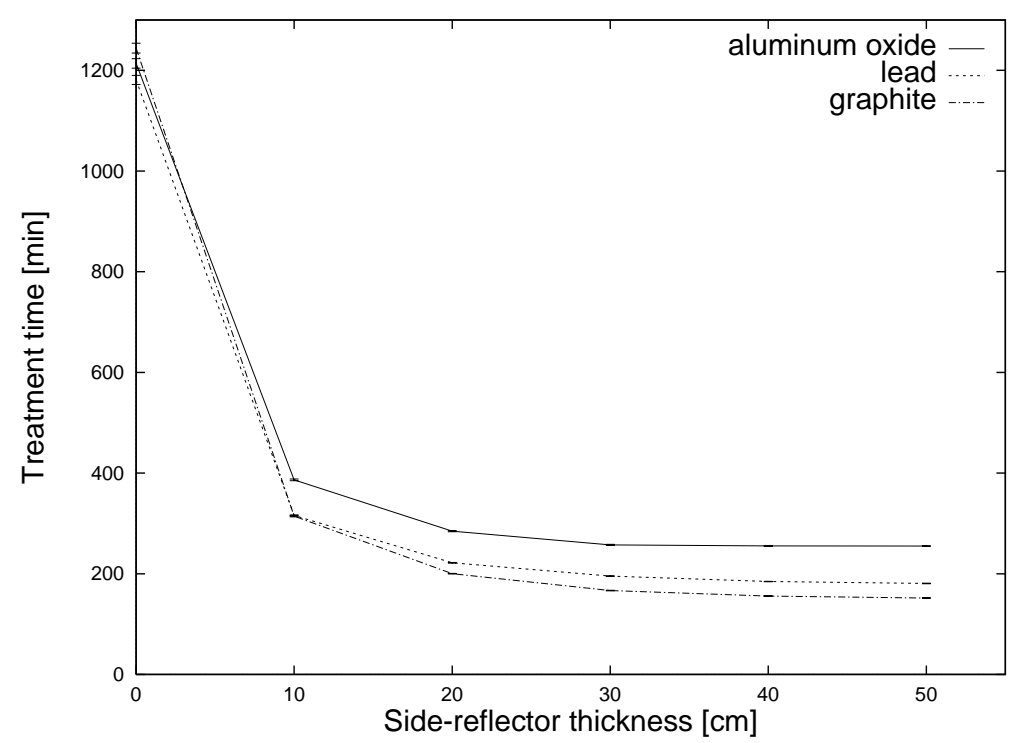

Figure 6.9: Side-reflector material and thickness parametric study. Treatment time as a function of the side-reflector material and thickness.

\subsubsection{Back-reflector design}

With the material and thickness of the side-reflector determined in Sec. 6.5.1, we analyze the effect of the back-reflector material and thickness. The same materials and thicknesses as in Sec. 6.5.1 are considered for the back-reflector. Figures 6.10 and 6.11

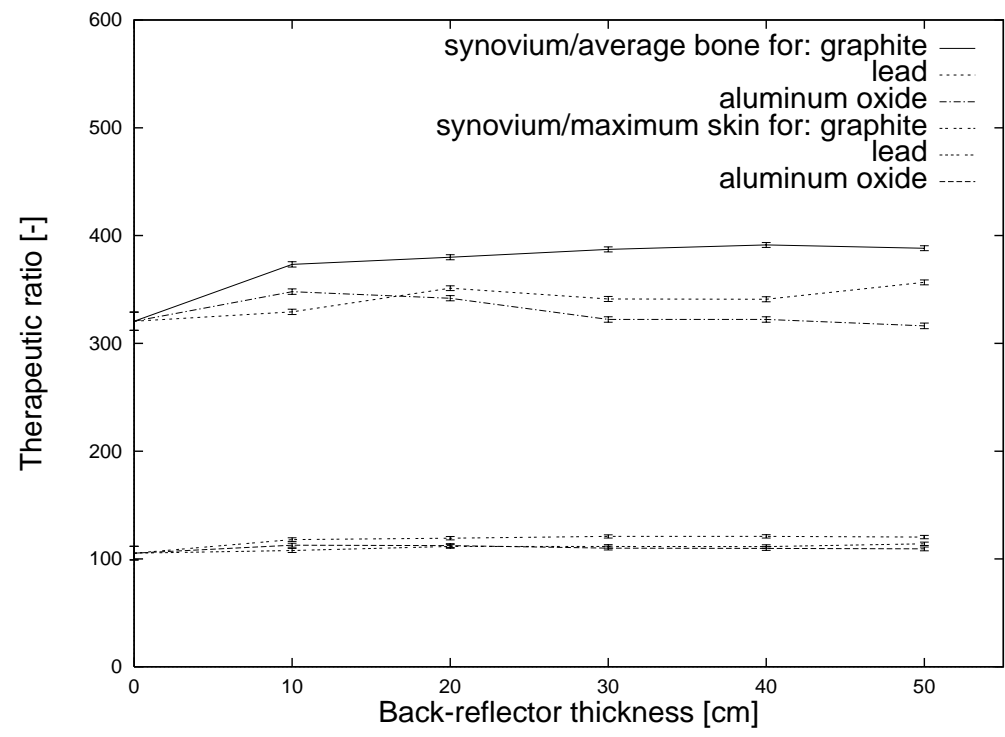

Figure 6.10: Back-reflector material and thickness parametric study. Therapeutic ratios as a function of the back-reflector material and thickness. 
show the results of this parametric study. The back-reflector thickness has no significant

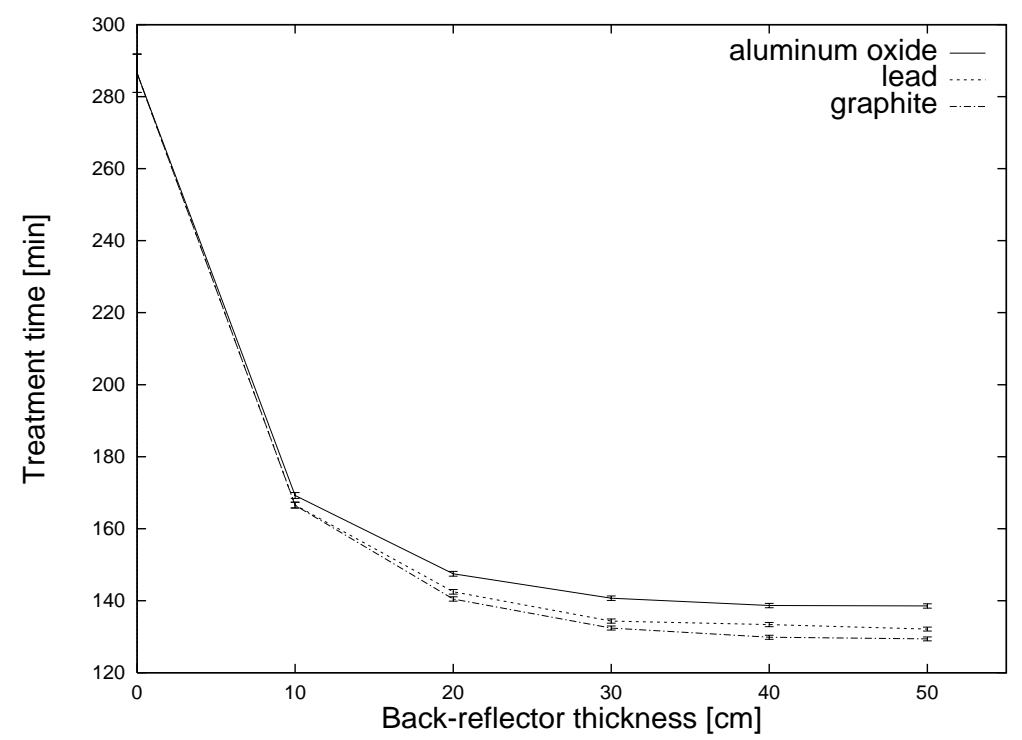

Figure 6.11: Back-reflector material and thickness parametric study. Treatment time as a function of the back-reflector material and thickness.

influence on the therapeutic ratios, while the treatment time decreases rapidly for backreflector thicknesses up to $30 \mathrm{~cm}$. Concerning the back-reflector material, graphite exhibits better characteristics than lead and aluminum oxide. A 30-cm-thick layer of graphite will be used further in Secs. 6.5.3 and 6.5.4 for the back-reflector.

\subsubsection{Optimization of moderator thickness}

With the 30-cm-thick graphite side- and back-reflectors, simulations have been carried out with different heavy water moderator thicknesses, from $0 \mathrm{~cm}$ to $100 \mathrm{~cm}$. The results are shown in Fig. 6.12. Both therapeutic ratios increase with the thickness for thicknesses up to $70 \mathrm{~cm}$. For larger thicknesses, the ratio of the synovium dose to average bone dose decreases slightly, while the ratio of the synovium dose to the skin dose remains constant. The treatment time increases exponentially with the thickness. A 50-cm-thick heavy water moderator gives simultaneously high therapeutic ratios and reasonable treatment times. This thickness will be used for the moderator diameter parametric study. Other materials were tried for the moderation but none of them had better characteristics than heavy water, and were not retained. 


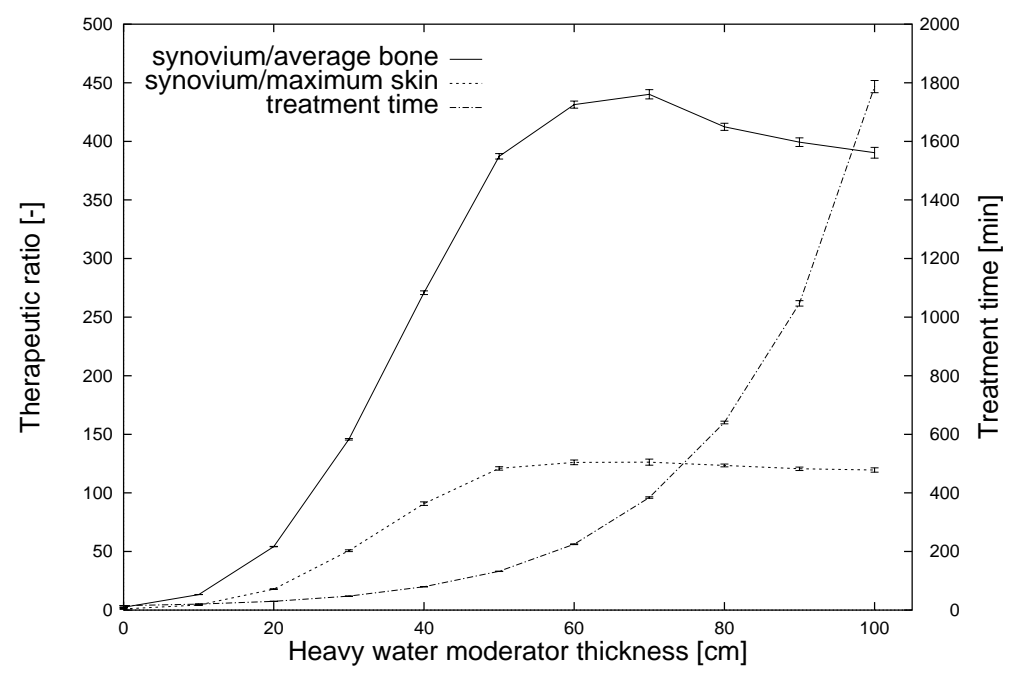

Figure 6.12: Therapeutic ratios and treatment time as a function of the heavy water moderator thickness.

\subsubsection{Optimization of moderator diameter}

The moderator diameter, which had been set to $35 \mathrm{~cm}$ initially and remained unchanged throughout the previous sections, has then been varied in the range $15 \mathrm{~cm}$ to $75 \mathrm{~cm}$, the results are shown in Fig. 6.13. The therapeutic ratios increase steeply up to a diameter of $35 \mathrm{~cm}$, after that the gains are less pronounced. The treatment time decreases

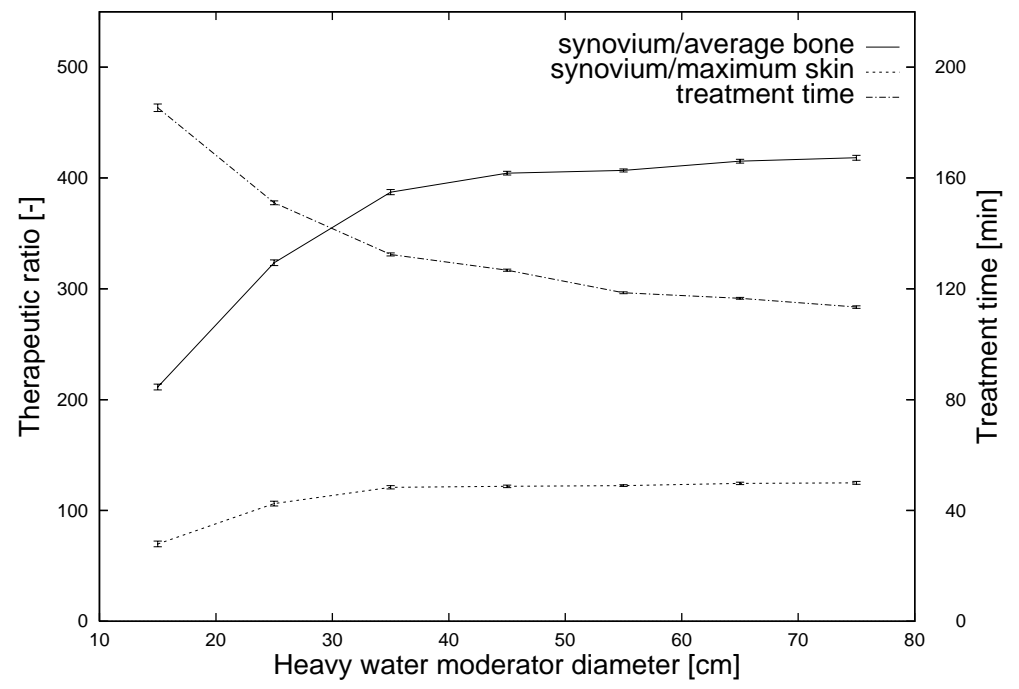

Figure 6.13: Therapeutic ratios and treatment time as a function of the heavy water moderator diameter. 
with increasing diameter. A diameter of $55 \mathrm{~cm}$ is considered optimal for both the treatment time and the therapeutic ratios.

To summarize the results obtained in the BSA optimization study, we conclude that a 50-cm-thick, 55-cm-diam heavy water moderator is optimal for the moderation of D-D neutrons. For the reflection of neutrons leaking out of the moderator, the heavy water is surrounded by a 30-cm-thick graphite side-reflector and preceded by a 30-cm-thick graphite back-reflector. Concerning the delimiter between the heavy water moderator and the patient knee, an 8-cm-thick layer of lithiated polyethylene reduces sufficiently radiation exposure to the patient organs other than the knee. A 5-cm-thick graphite layer located between the heavy water and the lithiated polyethylene layer reflects neutrons leaking out of the heavy water volume back to this volume and reduces neutron losses by neutron absorption in the lithiated polyethylene layer. A 3-cm-thick graphite collimator at an angle of $20^{\circ}$ is optimal to collimate the neutrons through the exit window. A 2-cm-thick layer of lithiated polyethylene (see Fig. 6.1) is left between the collimator and the knee in order to compensate for the lower neutron attenuation of the collimator material.

With this BSA, a full simulation has been performed and therapeutic ratios of $396 \pm$ 4 and $113 \pm 2$ for the synovium/average bone and synovium/maximum skin, respectively, were obtained. These ratios can be compared with the ones obtained using the dose-response database method, $407 \pm 2$ and $122 \pm 1$. With a D-D neutron source strength of $7 \times 10^{11}$ $\mathrm{n} / \mathrm{s}$, one can predict a treatment time of $2 \mathrm{~h}$ and $20 \mathrm{~min}$. Unless this long treatment time is reduced by an increase of the ${ }^{10} \mathrm{~B}$ concentration in the synovium, or by a neutron flux amplifier such as a fission plate converter, it will be a major obstacle for the use of the D-D reaction for BNCS.

\subsection{Moderation of D-T neutrons}

The $\mathrm{D}$ - $\mathrm{T}$ reaction was considered next because its neutron yield is more than one order of magnitude higher than D-D. Therefore, shorter treatment times can be expected using this reaction. The neutron yield at the target used in this study is $10^{14} \mathrm{n} / \mathrm{s}$. This corresponds to the neutron yield of a mainly monatomic 2-A mixed deuteron/triton beam bombarding titanium or scandium targets with an energy of $150 \mathrm{keV}$, see Sec. 7.2.3.

The first moderator tried for the moderation of D-T neutrons was similar to the one used for the D-D neutrons and was composed of a single layer of heavy water. Even 
with thick layers of heavy water, this moderator did not result in good therapeutic ratios. The reason for this is shown in Fig. 6.6, the elastic scattering cross section of heavy water for $14.1 \mathrm{MeV}$ neutrons is relatively low. An analysis of the neutron energy distribution after moderation of D-T neutrons by heavy water reveals the presence of two peaks, one thermal peak and one peak at energies greater than $10 \mathrm{MeV}$. Other materials were thus considered for moderation.

Neutron moderation for BNCT in Chapter 4 and Ref. [42] showed that lead, bismuth, iron, lithium fluoride enriched in the isotope ${ }^{7} \mathrm{LiF}$ and FLUENTAL ${ }^{(C)}$ [35] (mixture of $40 \%$ aluminium and $60 \%$ aluminium fluoride) used for moderation of D-D and D-T neutrons gave good neutron spectra for boron neutron capture therapy applications. Lead, bismuth and iron were particularly good for reducing the fast components of the neutron spectrum. Based on this study, a new moderator was designed for the moderation of 14 $\mathrm{MeV}$ neutrons. It is composed of a 20 -cm-thick layer of lead, followed by a 25-cm-thick layer of lithium fluoride ${ }^{7} \mathrm{LiF}$, a 45 -cm-thick layer of heavy water and a 1 -mm-thick layer of lead to attenuate the photon flux at the end of the moderation. The moderator diameter was initially set to $35 \mathrm{~cm}$. The side- and back-reflectors are 10 -cm-thick layers of lead. 10-cm-thick lithiated polyethylene layers surround the side-reflector and preceed the back-reflector. The delimiter between the moderator and the knee is identical to the one described in Sec. 6.5.

Figures 6.14 and 6.15 compare the therapeutic ratios and treatment times obtained using the full simulation and the dose-response database methods for different graphite side-reflector thicknesses, and for lead and graphite in the 5-cm-thick layer preceding the 8-cm-thick lithiated polyethylene layer in the delimiter. There is an overall good agreement between the trendlines shown in the figures for the two simulation methods. This demonstrates that the dose-response database method can be used for the optimization of beam-shaping assemblies for D-T neutrons. In general, the dose-response database method gives higher therapeutic ratios and shorter treatment times than the full simulation method. The lead delimiter gives slightly higher $(\sim 5 \%)$ therapeutic ratios than the graphite delimiter for side-reflector thicknesses between $10 \mathrm{~cm}$ and $30 \mathrm{~cm}$. Therefore, a lead delimiter will be used further in Secs. 6.6.1 through 6.6.4 for the BSA optimization. Since the graphite delimiter gives approximately the same results as the lead delimiter, it could easily replace it without affecting significantly the BSA performance in case the delimiter weight is of concern. 


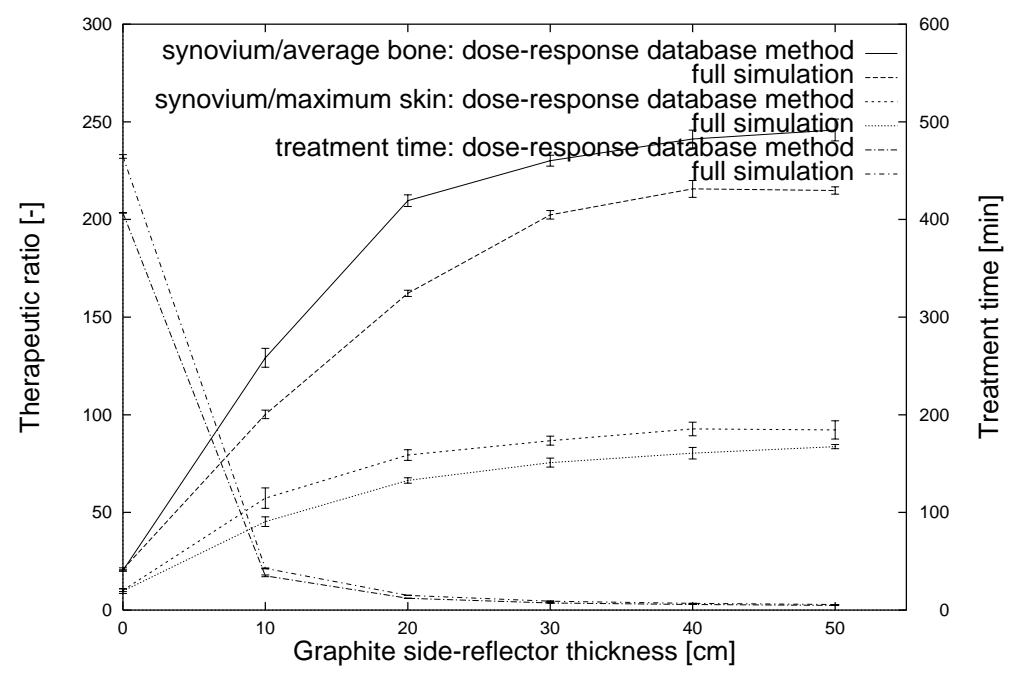

Figure 6.14: Comparison of treatment times and therapeutic ratios obtained with the doseresponse database and the full simulation methods using a lead delimiter.

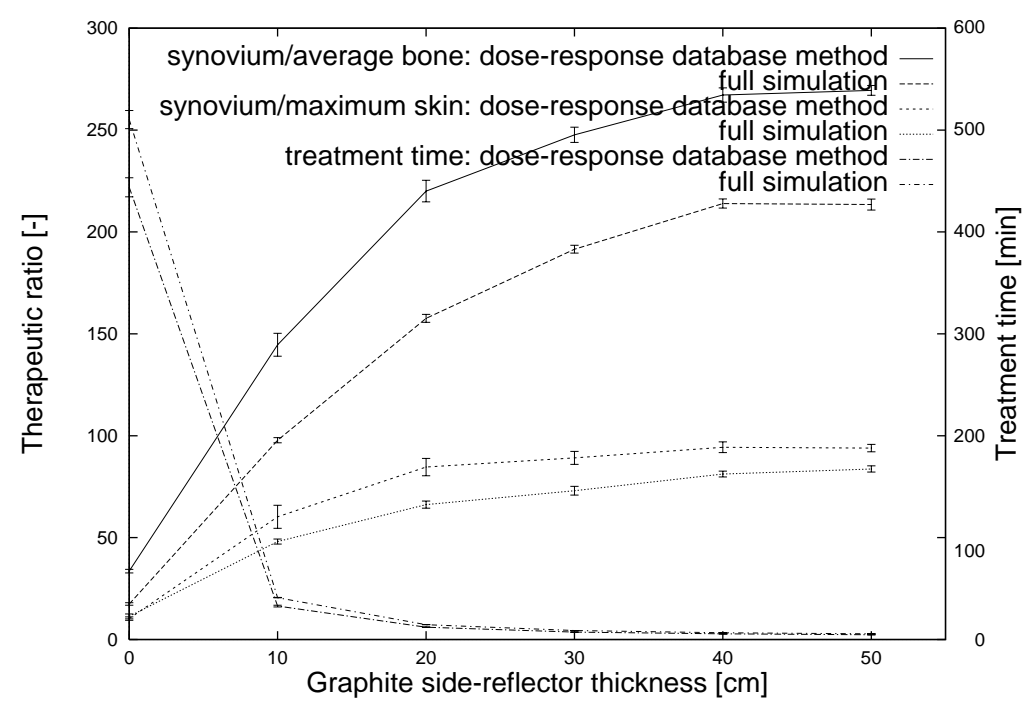

Figure 6.15: Comparison of treatment times and therapeutic ratios obtained with the doseresponse database and the full simulation methods using a graphite delimiter.

\subsubsection{Side-reflector design}

Using the lead delimiter, the side-reflector material and thickness are optimized. The materials considered for reflection are the same as the ones used for the D-D neutrons: lead, graphite and aluminum oxide. The results of this optimization are shown in Figs. 6.16 and 6.17 . 


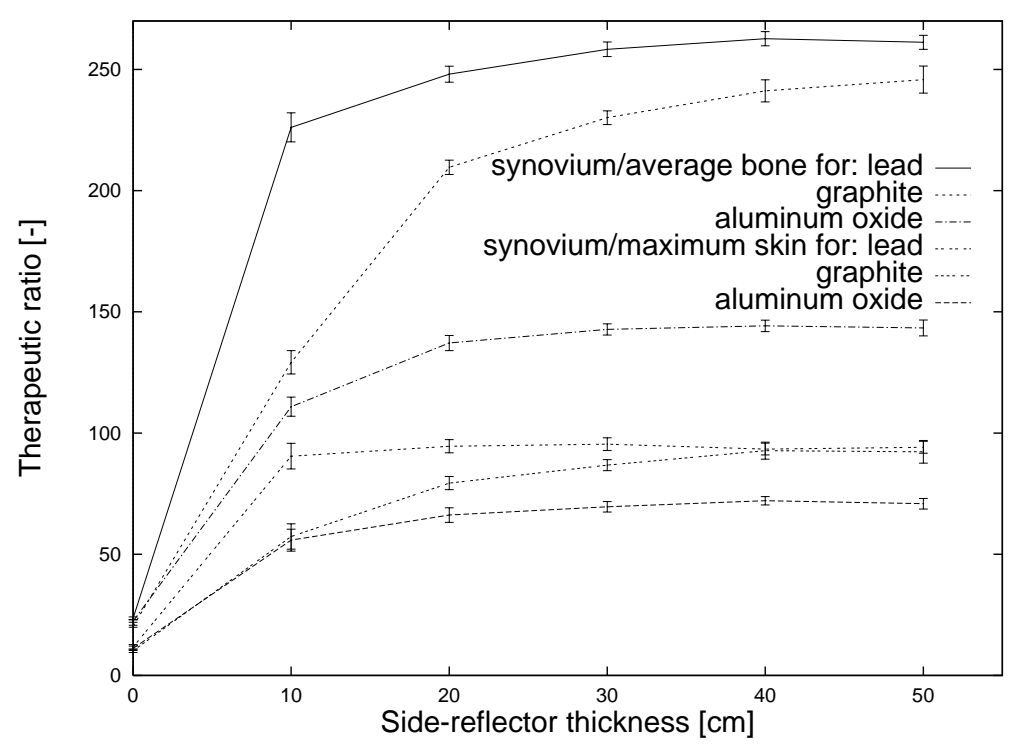

Figure 6.16: Side-reflector material and thickness parametric study. Therapeutic ratios as a function of the side-reflector material and thickness.

The therapeutic ratios increase and the treatment time decreases asymptoticallty with the thickness of the side-reflector. Aluminum oxide as a reflector results in very low therapeutic ratios due to the significant gamma-ray production in this material, which contaminates the neutron beam to be used for treatment. The highest therapeutic ratios

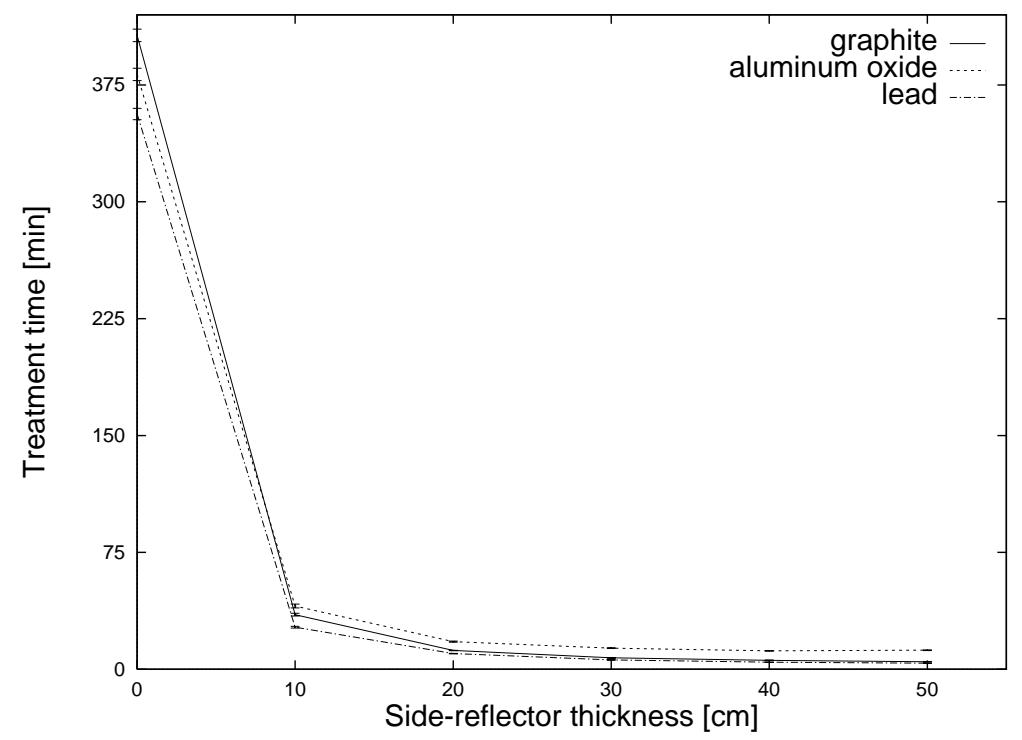

Figure 6.17: Side-reflector material and thickness parametric study. Treatment time as a function of the side-reflector material and thickness. 
and shortest treatment times are obtained with the lead side-reflector. Large thicknesses of lead are not necessary for high therapeutic ratios, $90 \%$ of the asymptotic values of the therapeutic ratios can be obtained using a 10-cm-thick lead side-reflector. Graphite gives therapeutic ratios about $10 \%$ lower than lead for thicknesses larger than $30 \mathrm{~cm}$. A $30-\mathrm{cm}-$ thick graphite side-reflector and a 10-cm-thick lead side-reflector give identical therapeutic ratios. However, since the density of lead is about 7 times higher than the density of graphite, the weight increase occuring when lead is used instead of graphite is not justified even though the side-reflector thickness is smaller. A 30-cm-thick graphite side-reflector will therefore be used further in this study. We keep in mind however that therapeutic ratios higher by about $10 \%$ could be obtained using heavy lead side-reflectors.

\subsubsection{Back-reflector design}

A brief analysis of the back-reflector material and thickness was also performed. The results are plotted in Figs. 6.18 and 6.19, where the therapeutic ratios and treatment times respectively, are plotted as a function of the back-reflector thickness. The ratios are basically constant over all thicknesses, while the treatment time decreases asymptotically with the thickness. Graphite is again preferred over other materials due to its lower density. A 30-cm-thick layer of graphite will be used further for the optimization of the moderator

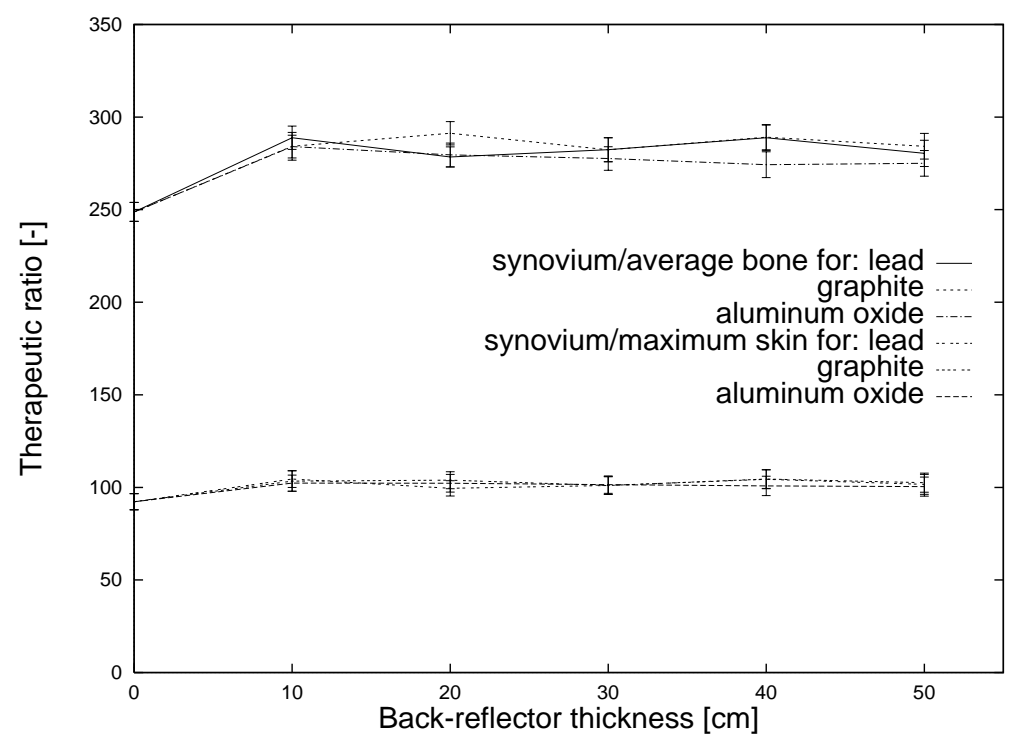

Figure 6.18: Back-reflector material and thickness parametric study. Therapeutic ratios as a function of the back-reflector material and thickness. 
diameter and thickness.

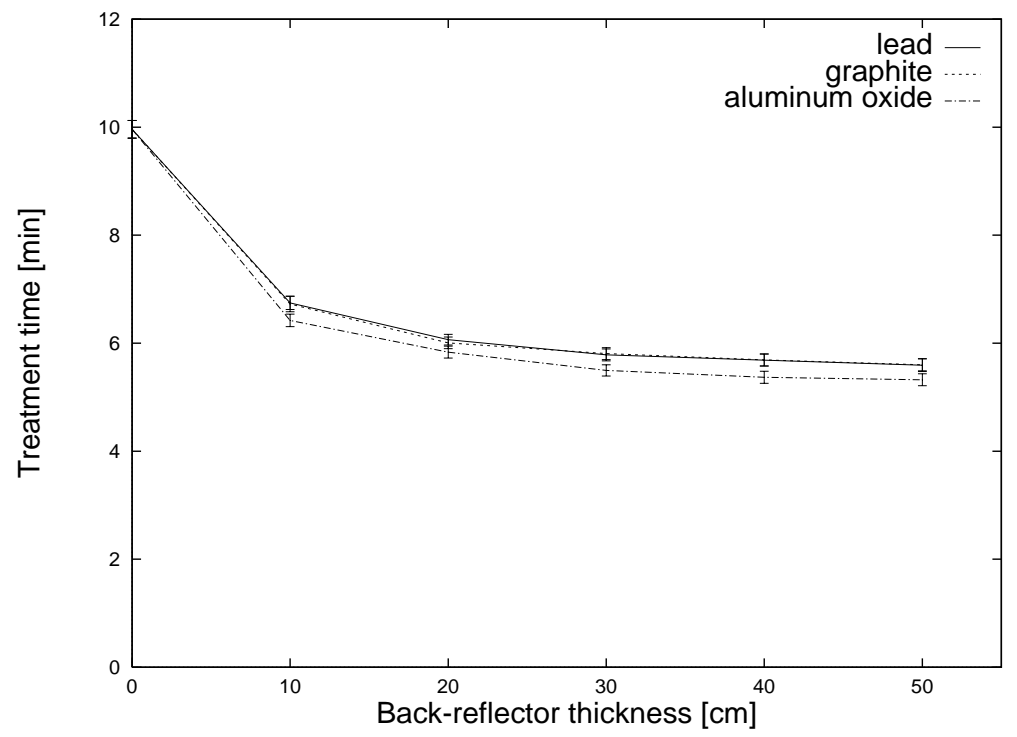

Figure 6.19: Back-reflector material and thickness parametric study. Treatment time as a function of the back-reflector material and thickness.

\subsubsection{Optimization of moderator diameter}

With the side-reflector and back-reflector described in Secs. 6.6.1 and 6.6.2, a moderator diameter parametric study is performed. The moderator diameter is varied from $15 \mathrm{~cm}$ to $85 \mathrm{~cm}$ in increments of $10 \mathrm{~cm}$. Both $30-\mathrm{cm}$-thick layers of lead and graphite were considered as side-reflectors for this study, since both materials gave similar results in the side-reflector thickness parametric study. The results of this study are shown in Fig. 6.20. The therapeutic ratios increase substantially with the moderator diameter and the treatment time decreases significantly. In the case of the graphite reflector for instance, the two therapeutic ratios increase from 89 and 234 for a 35-cm-diam moderator to 108 and 308 for a 55 -cm-diam moderator, and to 112 and 338 for a 85 -cm-diam moderator. For large moderator diameters, the difference between the graphite and the lead reflectors is not very pronounced, $5 \%$ to $10 \%$. 


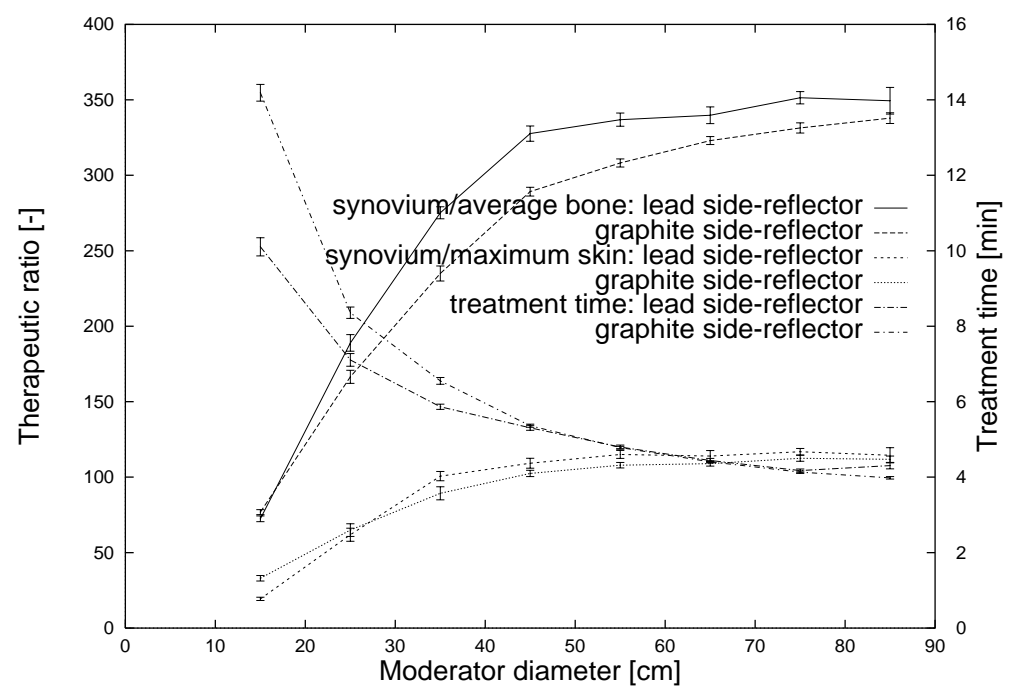

Figure 6.20: Moderator diameter parametric study. Therapeutic ratios and treatment time as a function of the moderator diameter and side-reflector material. A side-reflector thickness of $30 \mathrm{~cm}$ is used for all simulations.

\subsubsection{Optimization of moderator composition and thickness}

For the moderator thickness and composition study, a 55-cm-diam moderator with a graphite reflector will be used. One could obtain larger therapeutic ratios and better treatment times with even larger moderators, but $55 \mathrm{~cm}$ is a good compromise between moderator volume and thus weight, and moderator efficiency in terms of therapeutic ratios.

So far, the moderator was composed of a 20 -cm-thick layer of lead, followed by a 25 -cm-thick layer of lithium fluoride ${ }^{7} \mathrm{LiF}$, a 45 - $\mathrm{cm}$-thick layer of heavy water and a 1 mm-thick layer of lead to attenuate the photon flux at the end of the moderation. In order to see whether higher therapeutic ratios and shorter treatment times can be obtained with a better moderator, a parametric study was performed using the moderator above as a reference moderator and changing the lead, lithium fluoride and heavy water thick layer thicknesses by increments of $5 \mathrm{~cm}$. Iron was also tried as a moderator material but the high-energy photon production of this material did not make it attractive.

Figures 6.21 and 6.22 show the therapeutic ratios and treatment times respectively, as a function of the moderator material thickness increment. The vertical line is the reference moderator. The first data points of the lead curves represent the case of a moderator without lead. We will first examine the influence of lead in the moderation. The treatment time 


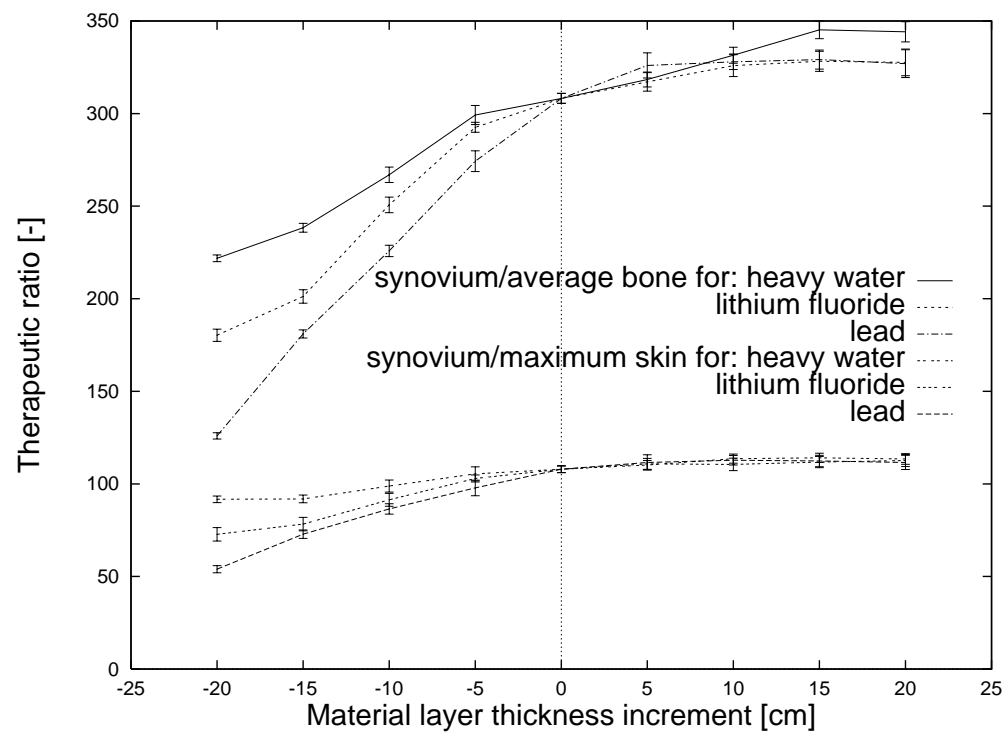

Figure 6.21: Moderator materials thickness parametric study. Therapeutic ratios as a function of moderator material thickness increment. The reference moderator is composed of a 20 -cm-thick layer of lead, followed by a 25 -cm-thick layer of lithium fluoride ${ }^{7} \mathrm{LiF}$, a 45-cm-thick layer of heavy water and a 1-mm-thick layer of lead.

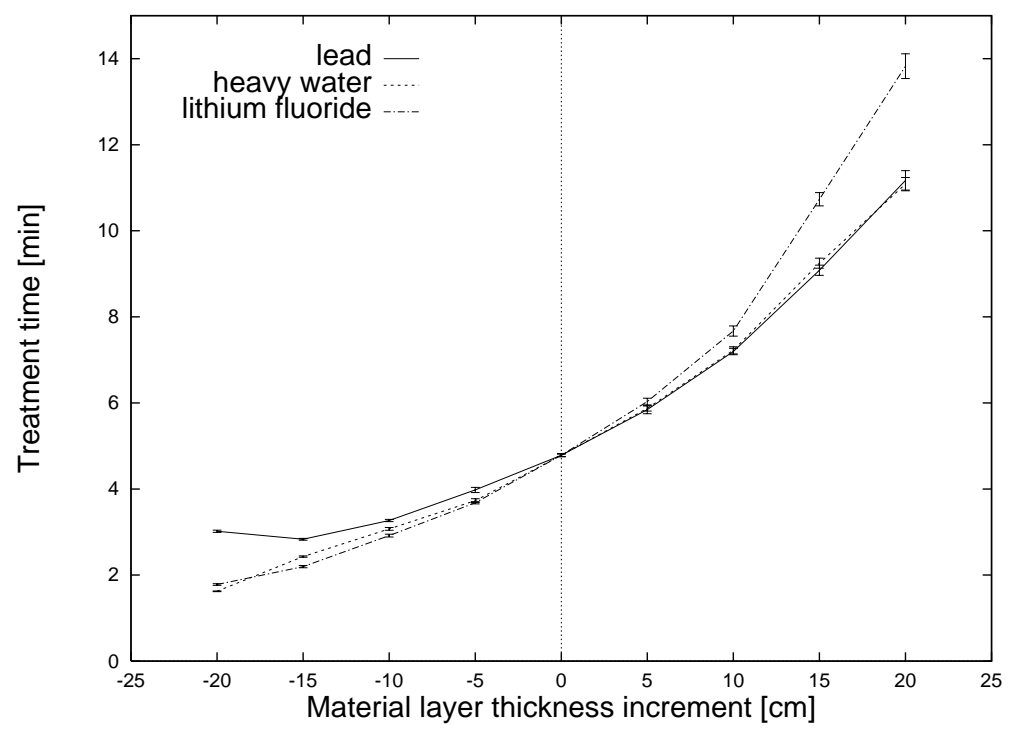

Figure 6.22: Moderator materials thickness parametric study. Treatment time as a function of moderator material thickness increment. The reference moderator is composed of a 20$\mathrm{cm}$-thick layer of lead, followed by a 25 -cm-thick layer of lithium fluoride ${ }^{7} \mathrm{LiF}$, a 45 -cm-thick layer of heavy water and a 1-mm-thick layer of lead. 
decreases when a 5-cm-thick layer of lead is added to the moderator without lead. This illustrates the effect of the $(n, 2 n)$ reactions occuring in lead at high neutron energies.

Therapeutic ratios increase steeply for lead layer thicknesses up to $25 \mathrm{~cm}$, and reach asymptotically a platean for larger thicknesses. From the therapentic ratios plotted in Fig. 6.21, it appears that an additional 5-cm-thick layer of lead would be beneficial for the treatment.

Concerning heavy water, the therapeutic ratios do not increase as rapidly with the thickness increment as lead or lithium fluoride. For very large heavy water thicknesses however, the therapeutic ratios reach values $5 \%$ higher than the two other materials. The behavior of lithium fluoride is somewhere between the ones of lead and heavy water. From the observations made in Figs. 6.21 and 6.22, it appears that replacing $5 \mathrm{~cm}$ of heavy water by $5 \mathrm{~cm}$ of lead would be beneficial for both the therapeutic ratios and the treatment times.

A simulation run was performed with $25 \mathrm{~cm}$ of lead, $25 \mathrm{~cm}$ of lithium fluoride and $40 \mathrm{~cm}$ of heavy water. The therapeutic ratios synovium dose to maximum skin dose and synovium dose to average bone dose were effectively higher and equal to $111.9 \pm 1.9$ and $324.6 \pm 3.3$ respectively. The treatment time decreases slightly from $4.78 \pm 0.03$ min to $4.72 \pm 0.05 \mathrm{~min}$.

A full simulation was then carried out to check the accuracy of these results. The therapeutic ratios for the full simulation run were $97.9 \pm 1.6$ and $293.6 \pm 3.4$, while the treatment time was $5.74 \pm 0.04 \mathrm{~min}$. The 10 to $15 \%$ overestimation of the therapentic ratios and $20 \%$ underestimation of the treatment time obtained with the dose-response database method are in good agreement with the differences observed in Fig. 6.14 and confirms that a full simulation is always necessary, after the BSA has been optimized with the doseresponse database method. A full simulation run was then performed with lead back- and side-reflectors instead of graphite ones, since we had noticed in Sec. 6.6.1 that this would enhance the therapeutic ratios and reduce the treatment time. The therapeutic ratios obtained were $111.0 \pm 2.4$ and $323.2 \pm 6.1$, while the treatment time was $4.03 \pm 0.04$ min. These treatment characteristics are $\sim 10 \%$ better than the ones for the graphite reflectors. Depending on whether weight is of concern, lead or graphite will be selected as a reflector material.

In summary, the best moderator that has been tried was a 25-cm-thick layer of lead followed by a 25 -cm-thick layer of lithium fluoride ${ }^{7} \mathrm{LiF}$ and a 40 -cm-thick layer of heavy water. This moderator is $55 \mathrm{~cm}$ in diameter and surrounded by a $30-\mathrm{cm}$-thick graphite 
layer to reflect neutrons which would otherwise leak out of the moderator. In order to reduce radiation exposure to the patient's organs other than the knee, a 8-cm-thick lithiated polyethylene delimiter is placed between the moderator and the patient's knee. A 5 -cm-thick lead layer is placed between the moderator and the polyethylene layer to reflect neutrons leaking out through this surface, see Fig. 6.1. The inside of the exit window in the polyethylene layer is lined with a 3 -cm-thick layer of lead at an angle of $20^{\circ}$ to collimate the neutrons to the knee. With this BSA, therapeutic ratios synovium/maximum skin and synovium/average bone of $97.9 \pm 1.6$ and $293.6 \pm 3.4$ were obtained, the treatment time was $5.74 \pm 0.04$ min. Lead back- and side-reflectors give in general $\sim 10 \%$ better results but are much heavier.

\subsection{Summary of results}

The neutron sources considered for boron neutron capture synovectomy were D-D and D-T, which emit 2.45 and 14.1 MeV neutrons. The neutron yields considered for the $\mathrm{D}$-D and D-T reactions were $7 \times 10^{11}$ and $10^{14} \mathrm{n} / \mathrm{s}$ respectively. The dose-response database method developed in Chapter 5 was used to accelerate the design of beam-shaping assemblies for BNCS. A 50-cm-thick, 55-cm-diam heavy water moderator surrounded by a graphite reflector is optimal for moderation of D-D neutrons. However, the treatment time was longer than $2 \mathrm{~h}$. It could potentially be reduced if the ${ }^{10} \mathrm{~B}$ concentration in the synovium is increased. For D-T neutrons, a 25-cm-thick layer of lead followed by a 25-cm-thick layer of lithium fluoride ${ }^{7} \mathrm{LiF}$ and a 40 -cm-thick layer of heavy water gives high therapeutic ratios and a treatment time of approximately $5 \mathrm{~min}$. In the case of $\mathrm{D}-\mathrm{T}$, an increase in the moderator assembly diameter significantly reduces the treatment time. 


\section{Chapter 7}

\section{Compact sealed high-intensity neutron generator}

\subsection{Introduction}

Recently, LBNL has developed a compact, sealed-accelerator-tube neutron generator capable of producing $10^{9}$ to $10^{10} \mathrm{D}$-T neutrons per second [43, 44]. The ion source, a miniaturized variation of earlier RF-driven multicusp ion sources, is designed to fit within a 5 -cm-diam borehole. Typical operating parameters include repetition rates up to $100 \mathrm{pps}$, with pulse widths between 10 and $80 \mu s$ (limited only by the available RF power supply) and source pressure as low as 5 mTorr.

The neutron production requirements estimated in the Chapters on BNCT and

BNCS are of the order of $10^{14} \mathrm{n} / \mathrm{s}$. This high neutron output would also be adequate for other applications, such as explosive detection in airports, mine detection, biological research, neutron radiography, etc. As we will see in Sec. 7.3 on the ion source, there are several new developments which enable the neutron tube to provide a higher neutron yield: (a) $\mathrm{H}^{+}$yields over $95 \%$ have been achieved using a 5-cm-diam RF-driven multicusp source. High monatomic species yields are essential for high neutron outputs; (b) The ion source could be operated at low gas pressures (1 to 2 mTorr). Low gas pressures are necessary to reduce both charge exchange and high-voltage breakdown in the accelerator column. These experimental findings together with recent ion source testing will enable one to develop a new generation of compact neutron generators based on the fusion of deuterium and 
deuterium (D-D), or deuterium and tritium (D-T).

In order to achieve a neutron yield of $10^{14} \mathrm{n} / \mathrm{s}$ over long periods of time using the D-T reaction, a large multicusp source together with a multi-aperture extraction system to produce an ion beam current of $2 \mathrm{~A}$, accelerated to $150 \mathrm{kV}$, and impinging on a well-cooled target is required. The main components of the sealed neutron tube are the ion source, the $150 \mathrm{kV}$ accelerator column, the water-cooled target and the vacuum system. Figure 7.1 shows a schematic diagram of the sealed neutron generator. It is a scale up version of the

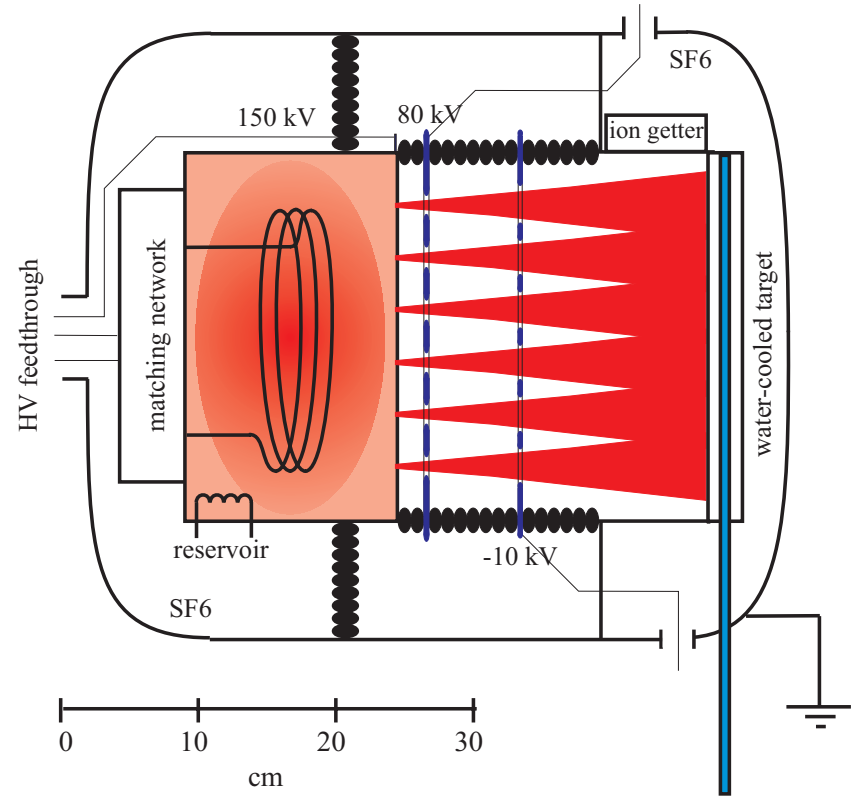

Figure 7.1: Schematic diagram of the sealed D-T neutron generator.

compact neutron tube that LBNL has recently developed.

In this system, the ion source, the accelerator and the target are all housed in a sealed metal container without external pumping. The gas pressure in the tube will be controlled by the reservoir element, which is typically a small diameter tungsten wire wrapped with zirconium. Just before operation, the reservoir element is heated electrically and provides enough gas pressure (deuterium and tritium) for the neutron tube operation. During operation, small amounts of deuterium and tritium gas are released from the target due to ion bombardment. The gas pressure in the tube is controlled by the reservoir element. After operation, deuterium and tritium gases return to the reservoir element and target, and the tube is essentially evacuated. The absence of gaseous tritium in the neutron tube 
between operations is a safety feature in case of mechanical failure during transportation.

Neutron yields of $10^{14} \mathrm{n} / \mathrm{s}$ can be achieved by bombarding a tritiated target with a 1 A deuteron beam at $120 \mathrm{keV}$. However, this neutron output deteriorates as deuterium coming from the beam slowly replaces the tritium occluded in the target. In order to maintain a constant neutron output, a beam-loading target will be used and the multicusp source will be operated with a $50 \%-50 \%$ mixture of deuterium and tritium. The concentration of deuterium and tritium in the target decreases with temperature. A large target area will be used in order to reduce the power density deposited by the impinging ions and to lower the target temperature. The lower temperature will result in a higher neutron output for a given ion beam current. With a $150 \mathrm{keV}$ and 2 A average mixed beam current hitting a well-cooled target, it is estimated that a neutron yield of $10^{14} \mathrm{n} / \mathrm{s}$ can be achieved over long periods of time.

The development of the neutron generator will be carried out in four successive steps, increasing the current from $1 \mathrm{~mA}$ to $15 \mathrm{~mA}, 150 \mathrm{~mA}$ and finally $1.5 \mathrm{~A}$. Neutron generation will take place in the last step only. In the three first steps, natural hydrogen will be used instead of deuterium and tritium in order to avoid health hazards related to radioactivity and neutron production. The first step has been completed, the second and third step are under development.

In this Chapter, we describe in detail the three main components of the neutron tube: the ion source, the accelerator column and the target.

\subsection{Target design}

The target is a copper substrate coated with a thin film of either scandium or titanium. Film thickness can range from 10 to $50 \mu \mathrm{m}$. The neutron production efficiency of target materials depends mainly on their capacity to retain deuterium and tritium, and on their stopping power. The more deuterium and tritium they can retain, the more nuclear fusion reactions can occur between incoming ions and occluded gas. The lower the stopping power of the material, the less energy the ions will lose interacting with it. Scandium and titanium form metal hydrides and can thus be used to produce neutrons from the D-T reactions. Due to their low atomic number, their stopping power is relatively low compared to other higher- $Z$ metals. Moreover, the ratio of hydrogen atoms to metal atoms - referred to as atomic ratio or $\mathrm{AR}$ in the text - for these metal hydrides can be as high as 2.0. These 
two properties make them the most efficient metal hydrides for neutron generation. Other metals form hydrides of atomic ratios up to 3.0 - and even 3.75 for thorium - but their higher $\mathrm{Z}$ and thus higher stopping power results in an overall lower neutron production efficiency.

\subsubsection{Thick-target neutron yield computations}

Even though titanium targets have been used extensively for neutron generation, the outcome of a thorough literature search for thick titanium target neutron yields resulted in only two papers, one report by Shope [45] and a paper by Kim [46]. Due to the lack of published experimental data for the stopping power of titanium in 1966, Shope estimated it by using two sets of data for elements that bracket titanium in atomic weight, namely, carbon and copper, and assuming that the atomic stopping power is proportional to the square root of the atomic weight. Using these estimated stopping powers, he computed neutron yields for deuteron and triton beams driven into titanium targets. Shope's calculations could be improved and completed using experimental data sets for the stopping power. Kim studied thick-target neutron yields for mixed beams driven into titanium targets. No information was found in the literature concerning neutron yields of scandium targets. In order to compare the efficiency of titanium and scandium as solid targets for neutron production, calculations of neutrons yields have been performed for deuteron beams, triton beams and mixed beams driven into these two metals using the stopping powers based on experimental data sets published by Andersen [47].

Calculations of neutron yields for a metal target onto which deuterium and tritium particles are bombarded, is very tedious because of the complexity of the concentration buildup inside the target. In steady state operation, the deuterium and tritium concentrations are believed to reach certain saturation values which depend on the beam current density, target material, and the target temperature. In the calculations which follow, it is assumed that the saturation concentrations are constant over the particle range, and that the target thickness is larger than the particle range.

For a deuteron beam of current $I$ and energy $E$ composed of monatomic and molecular species impinging on a target loaded with tritium, the total number of neutrons produced per second can be computed using the integral form of the thick-target yield 
equation:

$$
Y=\frac{A R \cdot I}{e} \sum_{k=1}^{3} k \cdot f_{k} \int_{0}^{E / k} \frac{\sigma_{D-T}(E)}{\frac{d E}{d x}(E)} d E \quad[n / s]
$$

where $A R$ is the atomic ratio of the tritium in the target, and $e$ is the electronic charge. The integrals represent the contributions of each ion species. They are weighted by their fraction $f_{k}$ and their number of nuclei $k$ per ion. $\sigma_{D-T}(E)$ is the neutron production cross section of the fusion reaction D- $\mathrm{T}$ and is taken from Shope [45]. $d E / d x(E)$ is the molecular stopping power of the target material loaded with tritium.

Similar equations can be written for deuteron beams bombarding targets loaded with deuterium, and for triton beams bombarding targets loaded with deuterium. The stopping power of the target material is assumed to follow Bragg's law of additivity, namely, that the molecular stopping power is the sum of the atomic stopping powers of the constituents:

$$
d E / d x_{M H_{A R}}=d E / d x_{M}+A R \cdot d E / d x_{H} \quad[e V / \text { molecule }]
$$

where $M$ indicates the metal occluder and $H$ the hydrogen isotope. The atomic stopping powers of deuterons and tritons in deuterium or tritium are taken directly from Shope [45]. They were computed from the well-established stopping power of protons in hydrogen [48, 49] and the experimentally established and accepted facts that (1) isotopes have the same atomic stopping power for the same incident particles and (2) isotopes of the same velocity lose energy at the same rate in the same material. For the stopping powers of deuterons in titanium and scandium, they were taken from Andersen [47]. Fact (2) was used to compute the stopping power of tritons in both metal occluders.

\subsubsection{Thick-target neutron yields for monoisotopic ion beams}

The integral in Eq. 7.1 is evaluated numerically for atomic ratios ranging from 0.2 to 2.0 and the results are plotted in Figs. 7.2 and 7.3 for the D-D nuclear reaction.

Neutron yields are traditionally computed per unit impinging ion beam current. In this study, we are interested in maximizing the neutron yield per unit ion beam power, because this last quantity determines the power required by the high-voltage power supplies, the heat load on the target and thus the heat exchanger requirements to cool down the target. Hence, the figures show the neutron yields per unit ion beam power versus ion beam energy. 


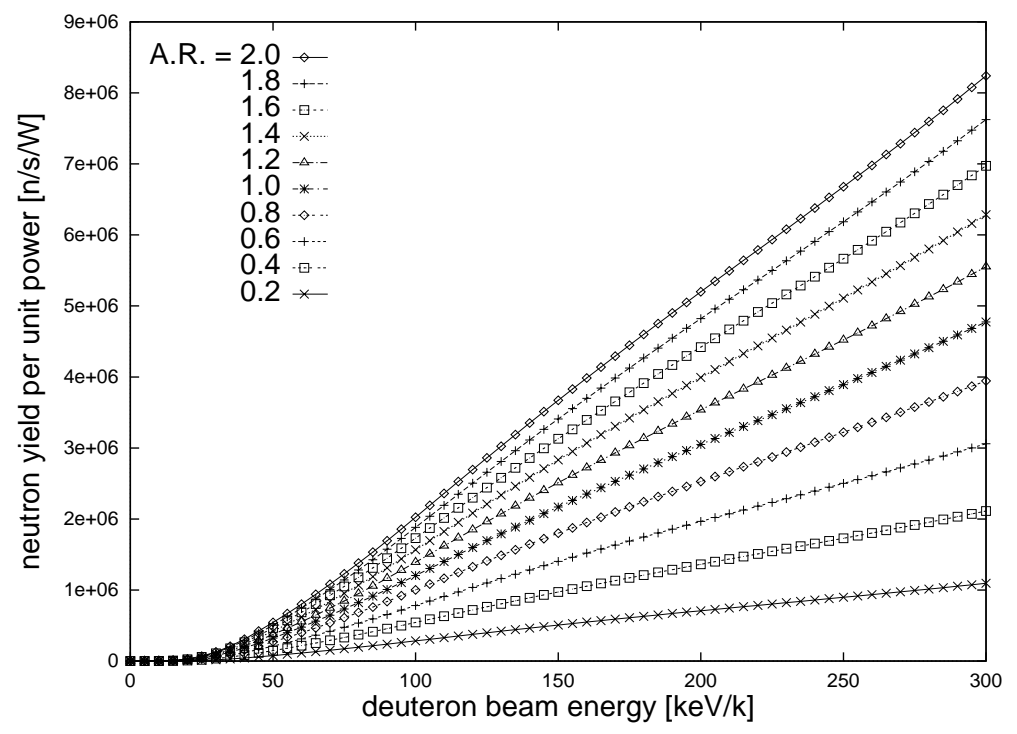

Figure 7.2: Neutron yield per unit beam power versus beam energy for deuteron beams impinging on a titanium target loaded with deuterium. $k$ in the units of the energy scale refers to the number of nuclei per ion.

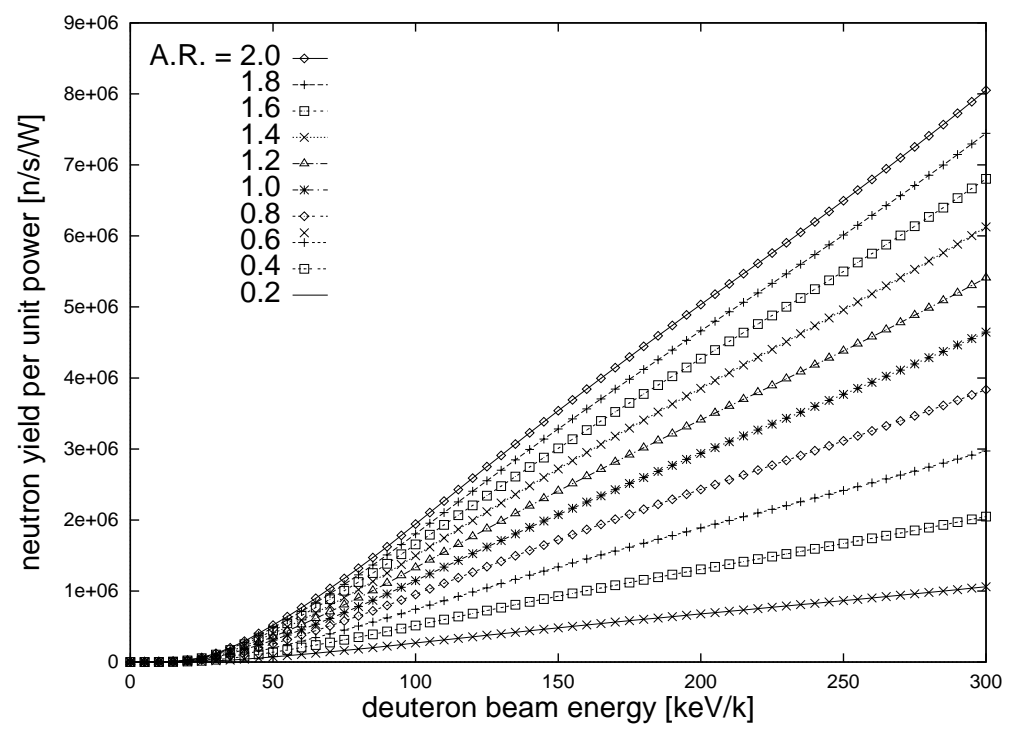

Figure 7.3: Neutron yield per unit beam power versus beam energy for deuteron beams impinging on a scandium target loaded with deuterium. $k$ in the units of the energy scale refers to the number of nuclei per ion.

The neutron yield increases with the deuteron beam energy for D-D. Its maximum is outside of the energy range of interest for compact neutron generators. Therefore, the operational voltage will strictly be determined by voltage holding in the accelerator column 
of the neutron generator.

The neutron yields per unit beam power for the D-T nuclear reaction are plotted in Figs. 7.4 and 7.5, and in Figs. 7.6 and 7.7 for the T-D nuclear reaction.

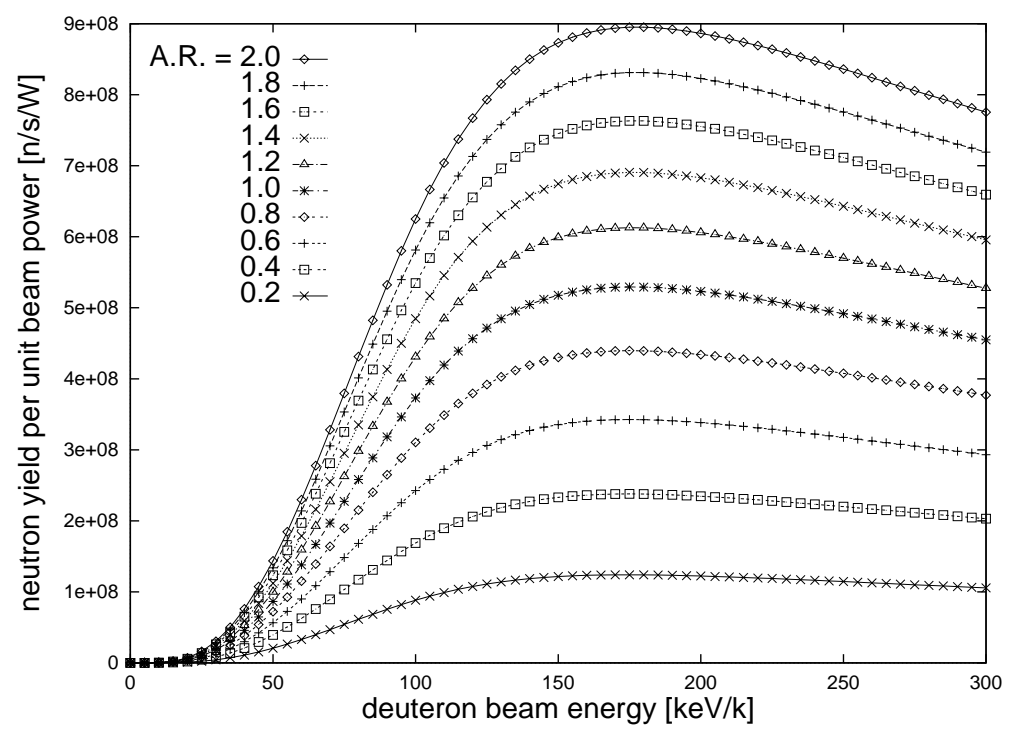

Figure 7.4: Neutron yield per unit beam power versus beam energy for deuteron beams impinging on a titanium target loaded with tritium. $k$ in the units of the energy scale refers to the number of nuclei per ion.

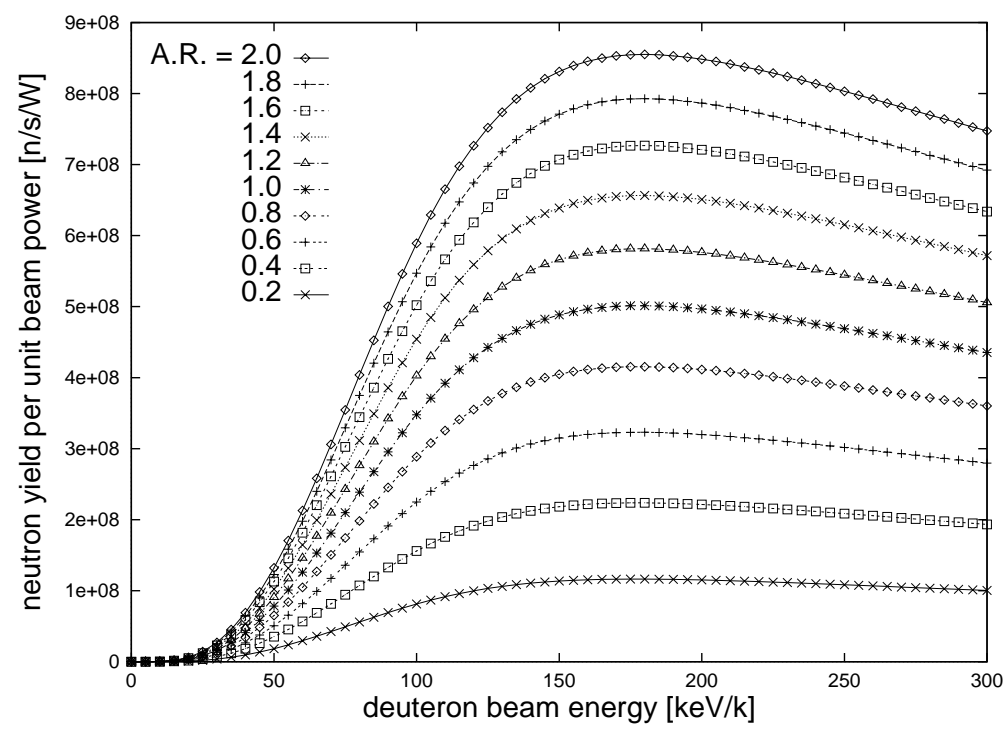

Figure 7.5: Neutron yield per unit beam power versus beam energy for deuteron beams impinging on a scandium target loaded with tritium. $k$ in the units of the energy scale refers to the number of nuclei per ion. 


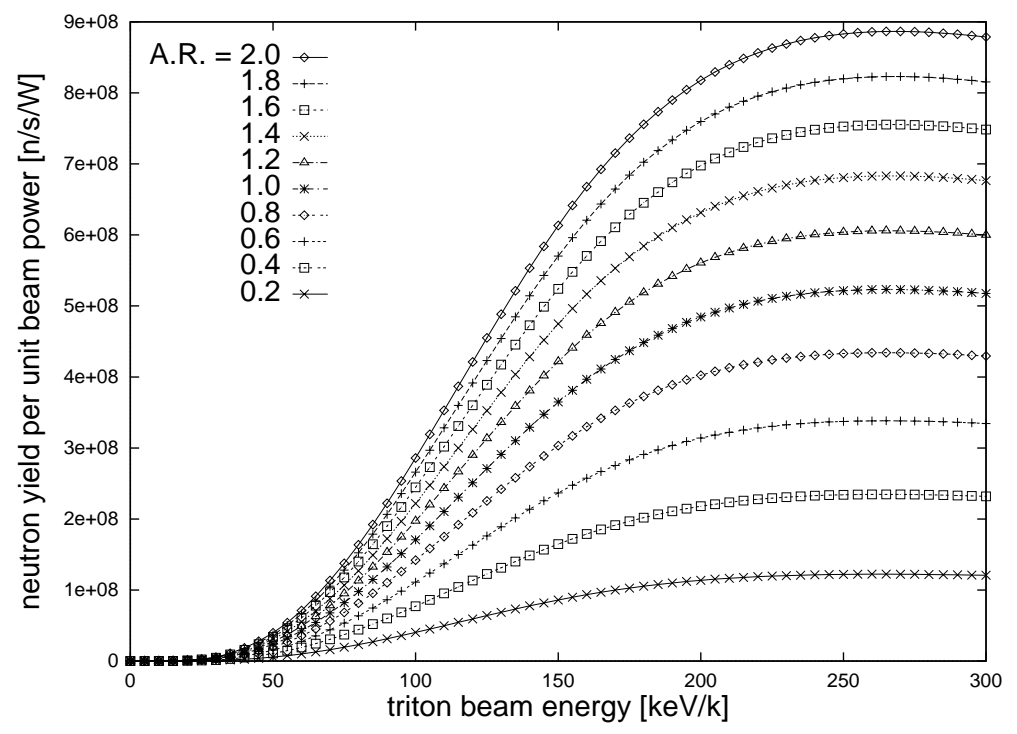

Figure 7.6: Neutron yield per unit beam power versus beam energy for triton beams impinging on a titanium target loaded with deuterium. $k$ in the units of the energy scale refers to the number of nuclei per ion.

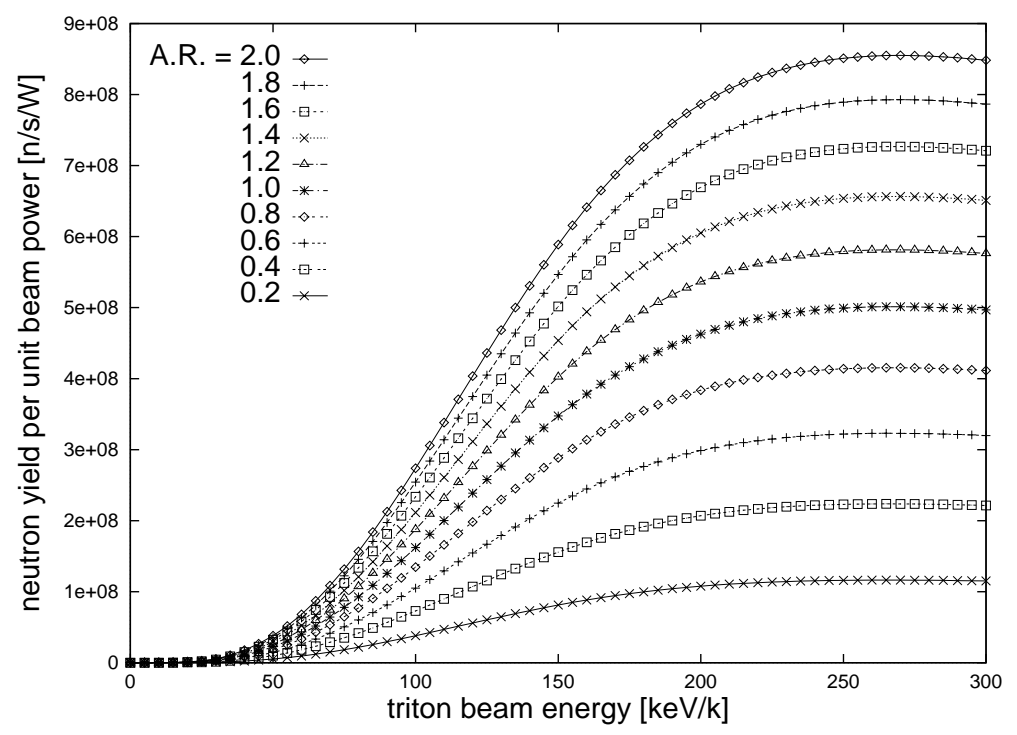

Figure 7.7: Neutron yield per unit beam power versus beam energy for triton beams impinging on a scandium target loaded with deuterium. $k$ in the units of the energy scale refers to the number of nuclei per ion.

For the D-T nuclear reaction, the highest neutron yields per unit ion beam power is obtained for a deuteron beam energy of $175 \mathrm{keV}$ for the titanium target and $180 \mathrm{keV}$ for the scandium target. These optimal energies are the same for all atomic ratios. The 
neutron yields per unit beam power decrease slowly around the optimal energies. Half of the neutron yields per unit beam power are still obtained at about $80 \mathrm{keV}$ for both targets and for all atomic ratios. For the T-D reaction, the optimal triton beam energy is 265 $\mathrm{keV}$ for titanium targets and $270 \mathrm{keV}$ for scandium targets. Since scandium has a higher stopping power than titanium, scandium targets have lower neutron yields than titanium targets. As shown in Figs. 7.4 and 7.5, the neutron yields increase with the atomic ratio.

The energy scales in the figures are in $\mathrm{keV}$ per number of nuclei $k$ per ion. The neutron yields per unit beam power for diatomic ion beams $D_{2}^{+}$and $T_{2}^{+}$or triatomic ion beams $D_{3}^{+}$and $T_{3}^{+}$can be computed from these figures by doubling and tripling respectively the energy scales. Thus, the optimal energy for a diatomic deuteron beam on a tritiated titanium target would be $350 \mathrm{keV}$ and its neutron yield per unit power would be identical to the neutron yield of the optimal $175 \mathrm{keV}$ monatomic deuteron beam. This higher energy is much more difficult to obtain with compact accelerators. Therefore, it is advantageous to produce mostly monatomic deuteron ions in the ion source. If the ion source provides mostly diatomic deuteron ions, the neutron output per unit power at $175 \mathrm{keV}$ will be less than half the neutron output for monatomic deuteron ion beams at the same energy. Similar conclusions can easily be drawn for diatomic triton beams, as well as triatomic deuteron and triton beams.

\subsubsection{Thick-target neutron yields for mixed ion beams}

With titanium and scandium targets loaded with tritium to an atomic ratio of 2.0 , neutron yields of about $10^{14} \mathrm{n} / \mathrm{s} / \mathrm{A}$ can be obtained with $120 \mathrm{keV}$ deuteron beams. However, this neutron yield deteriorates with time due to dilution of tritium in the target by deuterons from the ion beam. Indeed, a deuteron beam bombarding a tritiated target will gradually lead to mixed gas target operation and a decrease in neutron output with time. In order to maximize the lifetime of the target and to maintain a constant neutron output, a beam-loading target will be used and the multicusp ion source and accelerator column will be operated with a $50 \%-50 \%$ mixture of deuterium and tritium. This mode of operation solves the dilution problem. However, the neutron yield is lower.

For mixed beams composed of deuterons and tritons, the neutron yield at the target is the sum of several terms of the same form as Eq. 7.1: 


$$
\begin{aligned}
Y & =\frac{I}{e}\left[\sum_{k=1}^{3} k \cdot f_{D_{k}^{+}} \int_{0}^{E / k} \frac{A R_{T} \sigma_{D-T}(E)}{\frac{d E}{d x}(E)} d E \sum_{k=1}^{3} k \cdot f_{T_{k}^{+}} \int_{0}^{E / k} \frac{A R_{D} \sigma_{T-D}(E)}{\frac{d E}{d x}(E)} d E\right. \\
& +f_{D T^{+}}\left(\int_{0}^{2 \cdot E / 5} \frac{A R_{T} \sigma_{D-T}(E)}{\frac{d E}{d x}(E)} d E \int_{0}^{3 \cdot E / 5} \frac{A R_{D} \sigma_{T-D}(E)}{\frac{d E}{d x}(E)} d E\right) \\
& +f_{D_{2} T^{+}}\left(2 \int_{0}^{2 \cdot E / 7} \frac{A R_{T} \sigma_{D-T}(E)}{\frac{d E}{d x}(E)} d E \int_{0}^{3 \cdot E / 7} \frac{A R_{D} \sigma_{T-D}(E)}{\frac{d E}{d x}(E)} d E\right) \\
& \left.+f_{D T_{2}^{+}}\left(\int_{0}^{E / 4} \frac{A R_{T} \sigma_{D-T}(E)}{\frac{d E}{d x}(E)} d E 2 \int_{0}^{3 \cdot E / 8} \frac{A R_{D} \sigma_{T-D}(E)}{\frac{d E}{d x}(E)} d E\right)\right][n / s]
\end{aligned}
$$

where the $f \mathrm{~s}$ denote the fractions of each species in the ion beam, $A R_{D}$ and $A R_{T}$ refer to the ratios of occluded deuterium and tritium atoms respectively to metal atoms in the target.

The first term is the neutron yield produced by high-energy deuterons from the beam interacting with tritium in the target through $\mathrm{D}-\mathrm{T}$ reactions, the second term is related to T-D reactions. The other terms correspond to the interactions of $\mathrm{DT}^{+}, \mathrm{D}_{2} \mathrm{~T}^{+}$ and $D T_{2}^{+}$ions also present in the beam with deuterium and tritium in the target. The contributions of the D-D and T-T fusion reactions to the total neutron yield are neglected in this computation because the neutron production cross sections of the D-D and T-T reactions are much smaller than the ones of the $\mathrm{D}-\mathrm{T}$ and $\mathrm{T}-\mathrm{D}$ reactions.

In the case of mixed ion beams, the atomic ratio $A R$ in Eq. 7.2 is the ratio of the sum of deuterium and tritium occluded atoms in the target to metal occluder atoms. This is also the atomic ratio shown in Figs. 7.8 and 7.9 , where the neutron yields for mixed beams bombarding titanium and scandium targets are plotted versus ion beam energy.

The neutron yields per unit power for mixed beams are about twice lower than the ones for monoisotopic ion beams. The ion beam energy for which the neutron yield per unit power is the highest is about $230 \mathrm{keV}$ for titanium and $240 \mathrm{keV}$ for scandium. This energy is the same for all atomic ratios. 


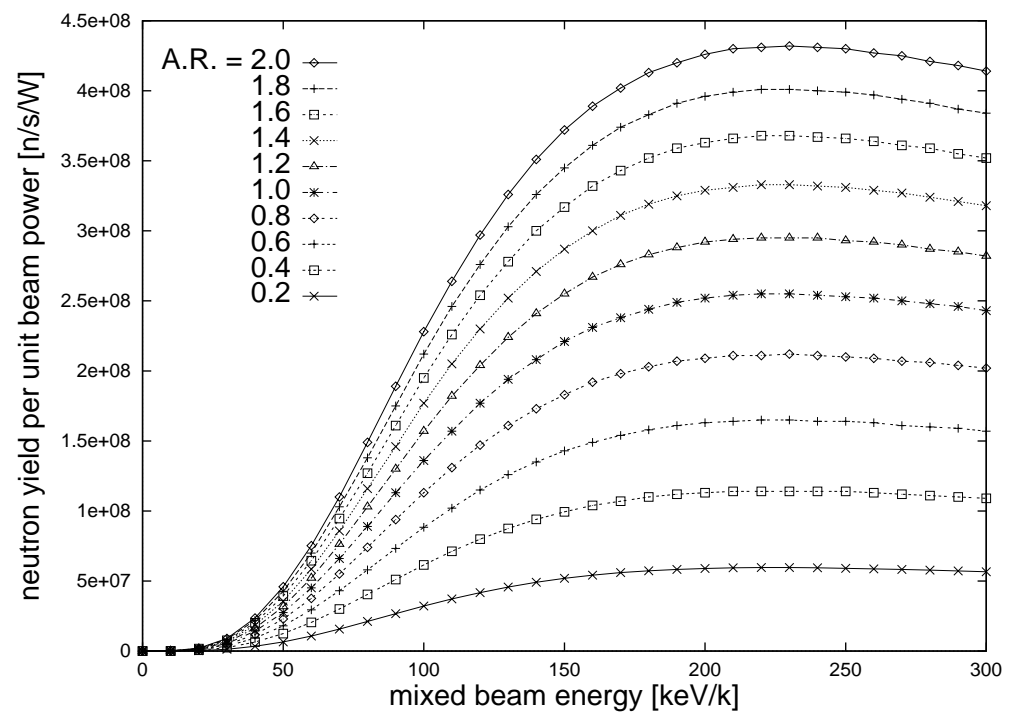

Figure 7.8: Neutron yield per unit beam power versus beam energy for mixed $50 \%$ deuteron $/ 50 \%$ triton beams impinging on a titanium target. $k$ in the units of the energy scale refers to the number of nuclei per ion.

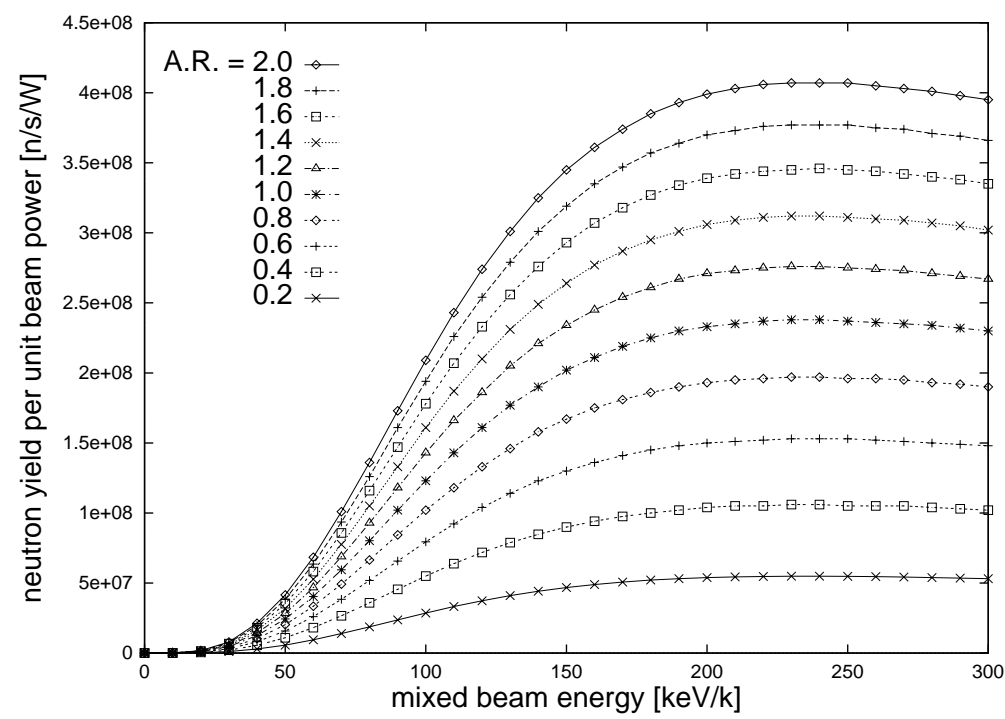

Figure 7.9: Neutron yield per unit beam power versus beam energy for mixed $50 \%$ deuteron $/ 50 \%$ triton beams impinging on a scandium target. $k$ in the units of the energy scale refers to the number of nuclei per ion.

\subsubsection{Effect of the monatomic/diatomic species fractions in the ion beam}

The effect of the monatomic fraction in the ion beam hitting the target is analyzed. For the sake of simplicity, the triatomic ion species are neglected in this analysis. 
The monatomic ion species fraction will be increased from $0 \%$ to $100 \%$ in increments of $20 \%$, while the diatomic ion species fraction will be decreased from $100 \%$ to $0 \%$ in decrements of $20 \%$. The effect of the ion species fractions will be studied for the two modes of operation which lead to a constant neutron output over time. The first one is the case of a deuteron beam bombarding a target loaded with deuterium, the second one is a mixed beam bombarding a target loaded equally with deuterium and tritium.

\section{Deuteron beam on deuterium-loaded target}

The effect of the monatomic deuteron fraction in a deuteron beam bombarding a target loaded with deuterium is first analyzed. The neutron yields per unit beam power are shown in Figs. 7.10 and 7.11 for titanium and scandium targets respectively. The neutron yield per unit beam power is again always higher for titanium targets than for scandium targets. Concerning the dependence on the deuteron beam composition, the neutron yield more than doubles when a $100 \%$ monatomic deuteron beam is used instead of a $100 \%$ diatomic deuteron beam. Regardless of the ion beam energy, it is advantageous to produce a high fraction of monatomic deuterons in the ion source in order to maximize the neutron output.

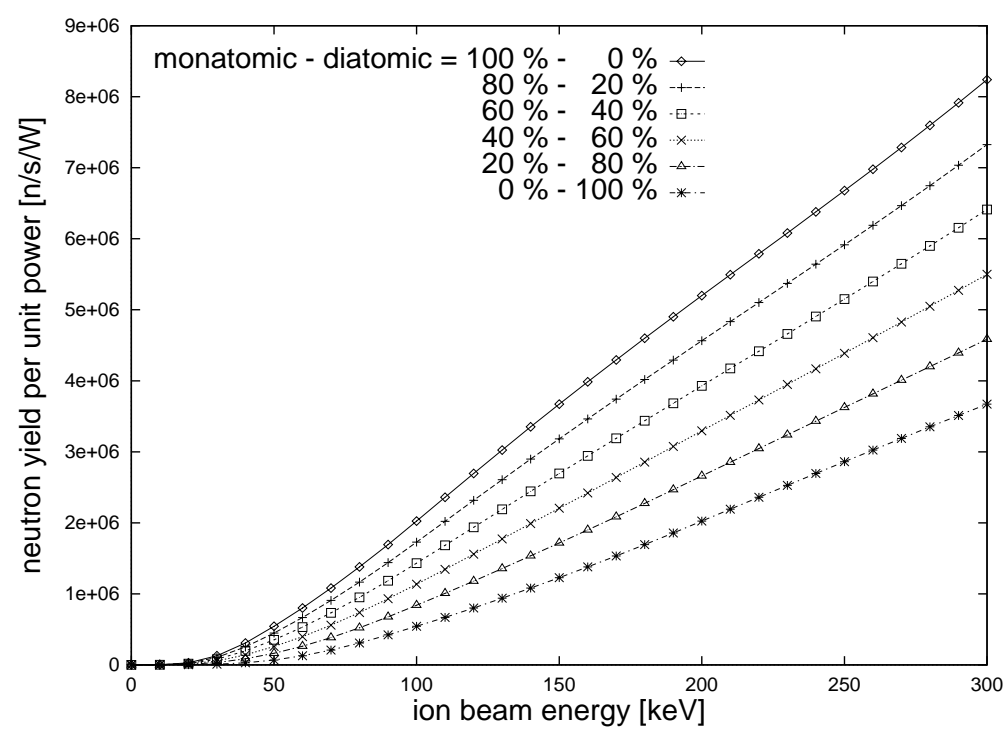

Figure 7.10: Neutron yield per unit beam power versus beam energy for multispecies deuteron beams impinging on a titanium target loaded with deuterium. The atomic ratio is set to 2.0 . 


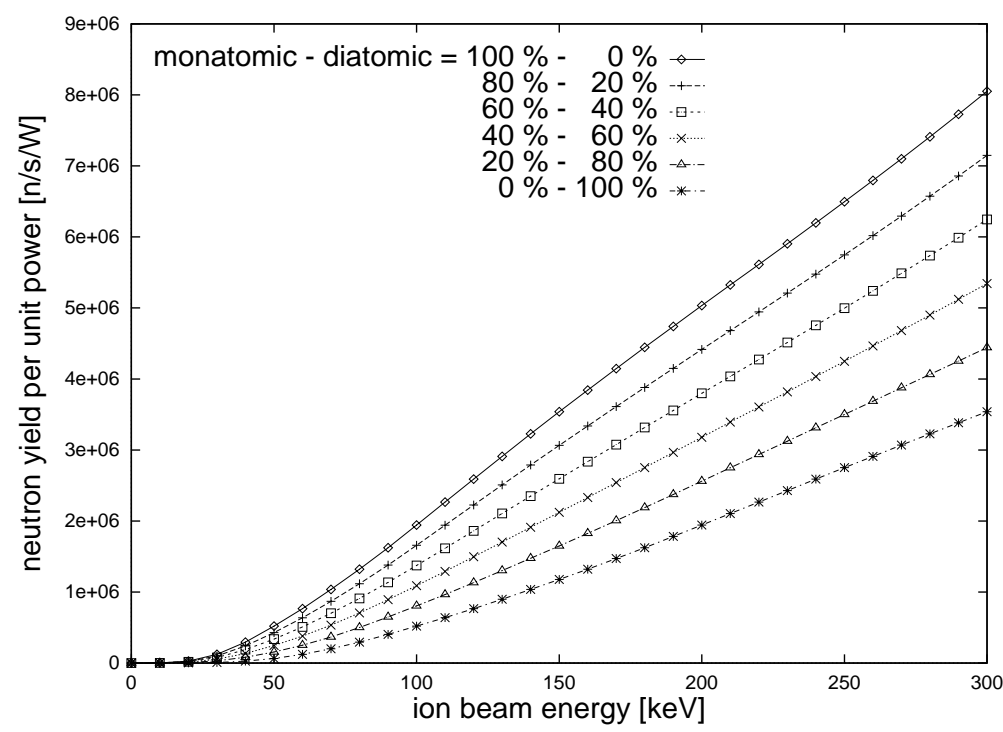

Figure 7.11: Neutron yield per unit beam power versus beam energy for multispecies deuteron beams impinging on a scandium target loaded with deuterium. The atomic ratio is set to 2.0 .

\section{Mixed beam on $50 \%$ deuterium $/ 50 \%$ tritium-loaded target}

For the case of a mixed beam bombarding a target loaded with both deuterium and tritium equally, the ion species fractions in the beam are assumed to be identical for deuterons and tritons, i.e., $f_{D^{+}}=f_{T^{+}}$and $f_{D_{2}^{+}}=f_{T_{2}^{+}}$. There are three different diatomic ion species, $D_{2}^{+}, T_{2}^{+}$and $D T^{+}$. We assume $f_{D_{2}^{+}}=f_{T_{2}^{+}}=0.5 f_{D T^{+}}$. Figures 7.12 and 7.13 show plots of the neutron yields per unit power for mixed beams of different compositions hitting titanium and scandium targets loaded with $50 \%$ deuterium/50\% tritium to an atomic ratio of 2.0. The neutron yield per unit power is independent of the beam composition at about $330 \mathrm{keV}$. The neutron yield per unit power is higher for monatomic ion beams than for diatomic ones below $330 \mathrm{keV}$ and lower above $330 \mathrm{keV}$. Therefore, depending on the ions species produced by the ion source, accelerator columns must operate at very different energies in order to maximize the neutron output.

The neutron output per unit power is maximized at $230 / 240 \mathrm{keV}$ for monatomic ions, at $460 / 480 \mathrm{keV}$ for diatomic ions, and at $690 / 720 \mathrm{keV}$ for triatomic ions bombarding titanium/scandium targets. The neutron output per unit beam power is lower at any other energies, regardless of the ion beam composition. If monatomic ion species are produced by the ion source, it is optimal to operate at $230 / 240 \mathrm{keV}$ and there is no gain to operate 


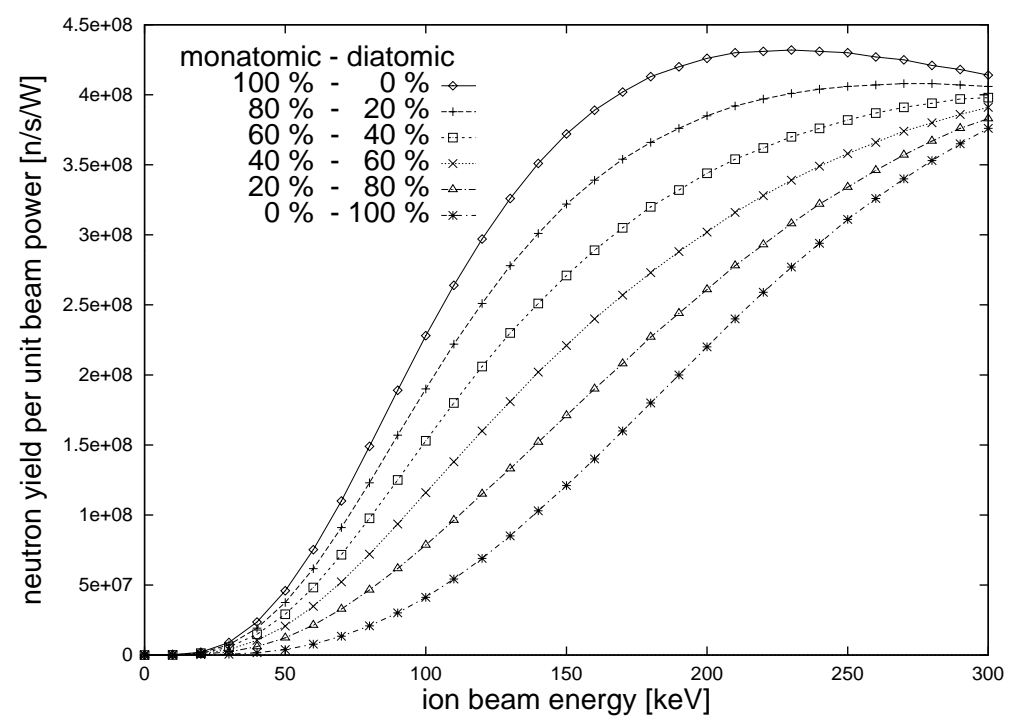

Figure 7.12: Neutron yield per unit beam power versus beam energy for multispecies, mixed $50 \%$ deuteron $/ 50 \%$ triton beams impinging on a titanium target loaded with $50 \%$ deuterium $/ 50 \%$ tritium. The atomic ratio is set to 2.0 .

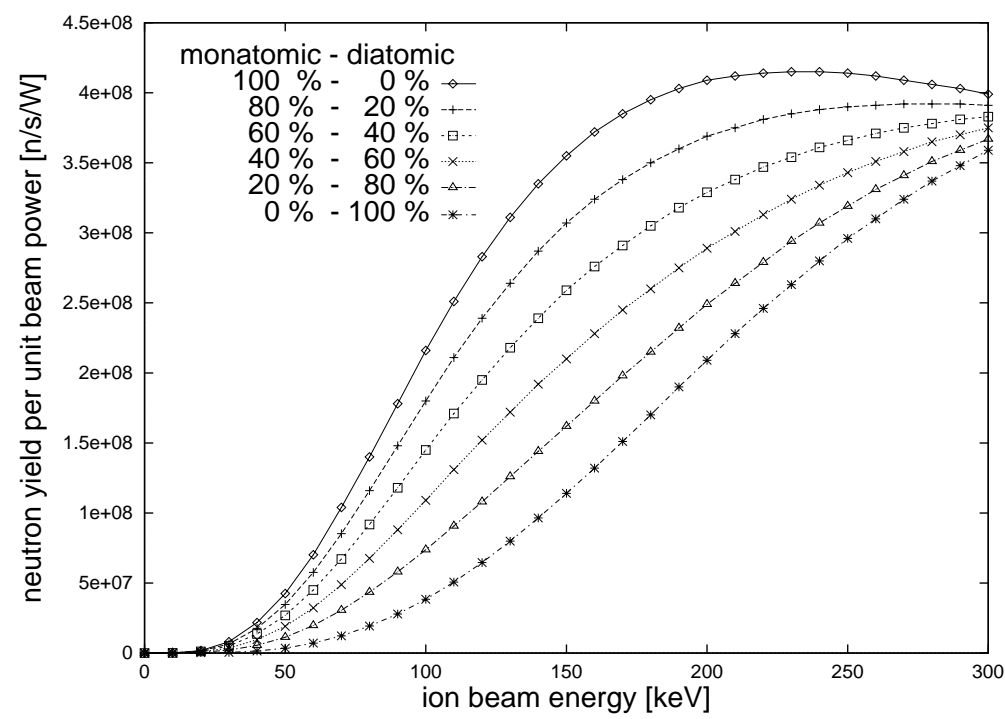

Figure 7.13: Neutron yield per unit beam power versus beam energy for multispecies, mixed $50 \%$ deuteron $/ 50 \%$ triton beams impinging on a scandium target loaded with $50 \%$ deuterium $/ 50 \%$ tritium. The atomic ratio is set to 2.0 .

at higher energies.

One might consider operating the generator at lower energies because of the difficulty to hold high voltages in compact accelerators. The neutron output per unit unit 
beam power decreases slowly from $230 / 240 \mathrm{keV}$ down to $150 \mathrm{keV}$, and more rapidly down to lower energies. Considering the size of the proposed compact neutron generator and the difficulties related to high voltage holding, the accelerator column will have to operate at energies lower than $200 \mathrm{keV}$. In order to maximize the neutron output at this energy, the ion source will have to produce a highly monatomic ion beam. This is the subject of Sec. 7.3.

\subsubsection{Thermodynamic stability of titanium and scandium targets}

A few words should be said about the maximum atomic ratios achievable for both scandium and titanium. As metal hydrides heat up, they release deuterium and tritium occluded gases. If the energy deposited by the ion beam is not removed, the temperature of the target rises, the atomic ratio and consequently the neutron yield decrease. To reduce the peak temperature to which the ion beam drives the target, the target is cooled by circulating water.

The atomic ratio of a metal in equilibrium with hydrogen gas can be determined by the gas pressure and the system temperature from PTC (pressure-temperature-composition) curves, which show the hydrogen gas pressure in equilibrium with metals as a function of the atomic ratio for different temperatures. For titanium and scandium at a given temperature, the hydrogen pressure increases rapidly with the atomic ratio in two ranges $A R<A R_{\text {lower }}$ and $A R>A R_{\text {upper }}$ where there are only single metal phases, and is constant between these two values where two metal phases are present. The two limits depend on the metal and the temperature, $A R_{\text {lower }}=0.6$ and $A R_{\text {upper }}=1.3$ for instance for scandium at $800^{\circ} \mathrm{C}$. The temperature corresponding to the plateau between $A R_{\text {lower }}$ and $A R_{\text {upper }}$ decreases with decreasing hydrogen pressure and can be calculated using the enthalpy and entropy of formation of the metal hydride using the following equation:

$$
\ln \left(p_{H_{2}}\right)=-\frac{2 \Delta S_{f}}{R}+\frac{2 \Delta H_{f}}{R T} \quad[-]
$$

where $p_{H_{2}}$ is the gas equilibrium pressure with the metal hydride in atmosphere, $\Delta S_{f}$ and $\Delta H_{f}$ are the entropy and enthalpy of formation of the metal hydrides per mole of hydrogen gas $\mathrm{H}_{2}, R$ is the universal gas constant and is equal to $8.3143 \mathrm{~J} / \mathrm{mol} / \mathrm{K}, T$ is the absolute temperature in Kelvin. Lieberman and Wahlbeck [50] measured the entropy and enthalpy of formation of scandium, they found $\Delta S_{f}=-145.5 \mathrm{~J} / \mathrm{mole}$ of $\mathrm{H}_{2}$ and $\Delta H_{f}=-200.8 \mathrm{~kJ} / \mathrm{K} /$ mole of $\mathrm{H}_{2}$. For titanium, Beavis [51] cites $\Delta S_{f}=-126.7 \mathrm{~J} / \mathrm{mole}$ of 
$\mathrm{H}_{2}$ and $\Delta H_{f}=-123.8 \mathrm{~kJ} / \mathrm{K} /$ mole of $\mathrm{H}_{2}$. From these values, on can conclude that scandium hydride is thermodynamically more stable than titanium hydride at high temperatures.

For titanium in equilibrium with hydrogen at $1 \mathrm{~m}$ Torr for instance, the equilibrium temperature of the platean is $244^{\circ} \mathrm{C}$, while it is $505^{\circ} \mathrm{C}$ for scandium. The atomic ratio will be higher than $\sim A R_{\text {upper }}$ for lower temperatures, and lower than $\sim A R_{\text {lower }}$ for higher temperatures. Therefore, it is essential to keep the temperature of the target reasonably low by an appropriate cooling system.

Very little information is available on PTC curves at low temperatures, but from these first considerations, we conclude that higher atomic ratios can be obtained with scandium hydride than with titanium hydride, especially for target temperatures of a few hundred degrees. If the atomic ratio is 1.9 for scandium and 1.6 for titanium, higher neutron yields will be obtained with scandium than with titanium. The neutron yields will be measured experimentally for both target materials.

\subsubsection{Target lifetime}

We have already seen that the use of a mixed ion beam and a target loaded with equal parts of deuterium and tritium eliminates the dilution problem. Mixed beam operation has the advantage of stable long term performance with the disadvantage of producing about a factor two lower neutron yield than a pure deuterium beam accelerated onto a target loaded with tritium.

Another factor that affect the lifetime of the target is sputtering. The sputtering rate of a $300 \mathrm{keV}$ deuteron beam on a $\mathrm{TiT}_{2}$ target has been measured to be about $0.65 \times 10^{-3}$ $\mathrm{mg} / \mathrm{C}$ [52]. Same rates were measured for $\mathrm{TiD}_{2}$ targets. The surface density of a $10-\mu \mathrm{m}-$ thick titanium layer is about $4.5 \mathrm{mg} / \mathrm{cm}^{2}$. If it is bombarded by a $1.5 \mathrm{~mA} / \mathrm{cm}^{2}$ deuteron beam, it will be completely sputtered in about $1300 \mathrm{~h}$. Since the thickness of the titanium layer will be in the range 10 to $50 \mu \mathrm{m}$, sputtering will not limit the lifetime of the target.

Eventually, one should consider the helium gas buildup in the system. Due to (i) the decay of tritium in the reservoir element and the target, (ii) the nuclear reactions producing an helium atom for each neutron produced, the neutron generator lifetime is limited by the helium gas buildup. We can easily compute that the pressure increase due to (ii) only in a $0.06 \mathrm{~m}^{3}$ sealed container for $1300 \mathrm{~h}$ of operation at $10^{14} \mathrm{n} / \mathrm{s}$ is about 240 mTorr. At these high gas pressures, the ion source can no longer function and the accelerator 
column will break down. Concerning (i), a target of surface area $1000 \mathrm{~cm}^{2}$ covered with $4.5 \mathrm{mg} / \mathrm{cm}^{2}$ of TiDT contains about $0.13 \mathrm{~g}$ of tritium. After complete decay, 0.04 moles of helium will be present in the system, resulting in even higher gas pressures, of the order of 13 Torr. An ion getter pump will be used to trap the helium gas. It can be turned on when the tube is not operating, i.e., when deuterium and tritium are evacuated from the tube and residing in the reservoir element.

\subsubsection{Summary of results}

For D-D, neutron yields of about $7 \times 10^{11} \mathrm{n} / \mathrm{s}$ can be obtained with a 1.5 - A, 150$\mathrm{keV}$ mainly monatomic deuteron beam bombarding deuterated targets. Neutron yields of about $10^{14} \mathrm{n} / \mathrm{s}$ can be obtained with a $1-\mathrm{A}, 120-\mathrm{keV}$ deuteron beam bombarding a tritiated targets. In the case of $\mathrm{D}-\mathrm{T}$ however, this neutron output deteriorates over time due to the replacement of tritium occluded in the target by deuterium coming from the beam. In order to maintain a constant neutron output, mixed beams composed of $50 \%$ deuterons / $50 \%$ tritons will be used for neutron production. From thick target neutron yield computations, $230-\mathrm{keV}$ mixed beams lead to the highest neutron yields per unit beam power. However, because of difficulties related to high-voltage holding in compact accelerator columns, the neutron generator will operate at energies lower than $200 \mathrm{keV}$. One can estimate a neutron production of approximately $10^{14} \mathrm{n} / \mathrm{s}$ for a $150-\mathrm{keV}, 2-\mathrm{A}$ mixed beam bombarding titanium and scandium targets. Neutron yield computations for ion beams with different monatomic/diatomic species fractions showed that the neutron output increases rapidly with the monatomic ion species fraction in the beam in the energy range of 150 to 200 $\mathrm{keV}$. It was also shown that low target temperatures are beneficial for the neutron yield. A cooling system for the target is therefore advised. Due to the greater thermal stability of scandium hydride, scandium targets lead to greater neutron yields than titanium targets, especially at high temperatures.

\subsection{Ion source}

The multicusp ion source will be a $30-\mathrm{cm}$-diam cylindrical stainless-steel chamber surrounded with columns of samarium-cobalt magnets. The plasma is produced by RF induction discharge. In order to deliver RF power to the plasma, a coupler in the form of a multi-turn induction coil is used. The RF power supply is a broad band power amplifier 
driven at $13.56 \mathrm{MHz}$ by a signal generator. To maximize the neutron output at the target, it is necessary to produce high $D^{+}$and $T^{+}$fractions in the extracted beam. Experiments have been carried out with a prototype 5-cm-diam ion source to determine the distribution of hydrogen ion species. The extractable ion beam current was also measured in order to design the accelerator column for the extraction. Ion distribution and current density measurements are the subject of Secs. 7.3.3 and 7.3.4. They were evaluated for different magnet configurations, which included the cases with and without a magnetic filter.

\subsubsection{Description of the prototype ion source}

A photograph of the prototype RF-driven ion source used for measurements of ion species distributions and ion beam currents is shown in Fig. 7.14. The ion source is $5.0 \mathrm{~cm}$

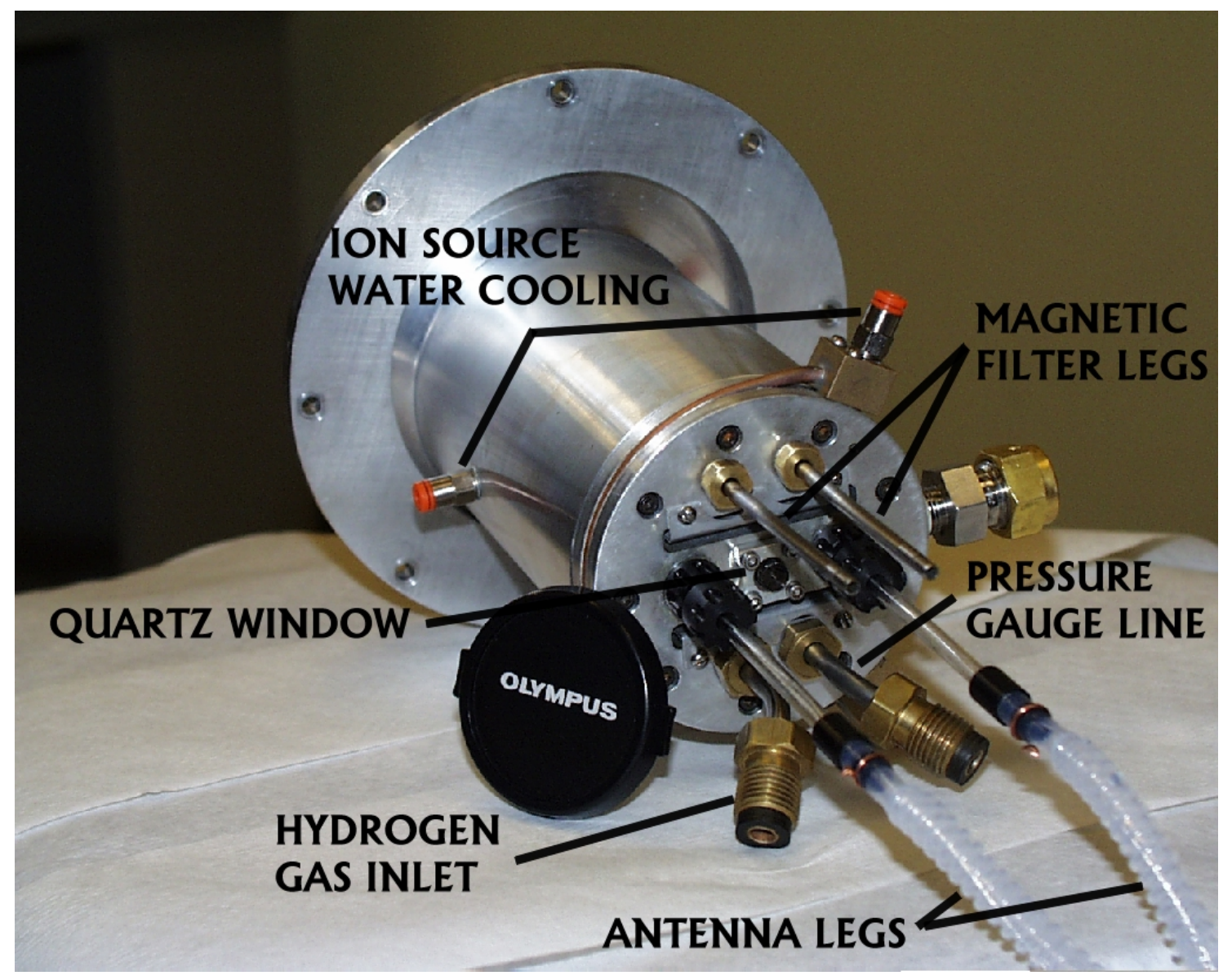

Figure 7.14: Ion source with quartz antenna and magnetic filter. 
in diameter and $11.7 \mathrm{~cm}$ long. The body is water-cooled by low-conductivity water. It is closed off at both ends by a plasma electrode on one side and a back flange on the other. The gas inlet, a pressure gauge, the feed-throughs for the RF antenna and the magnetic filter are installed in the back flange. The back flange is also equipped with a small diameter quartz window to permit viewing of the plasma in the ion source during operation.

Figure 7.15 shows a schematic diagram of the ion source, both in cross-section and end views. The prototype design incorporates several noteworthy features. Plasma con-

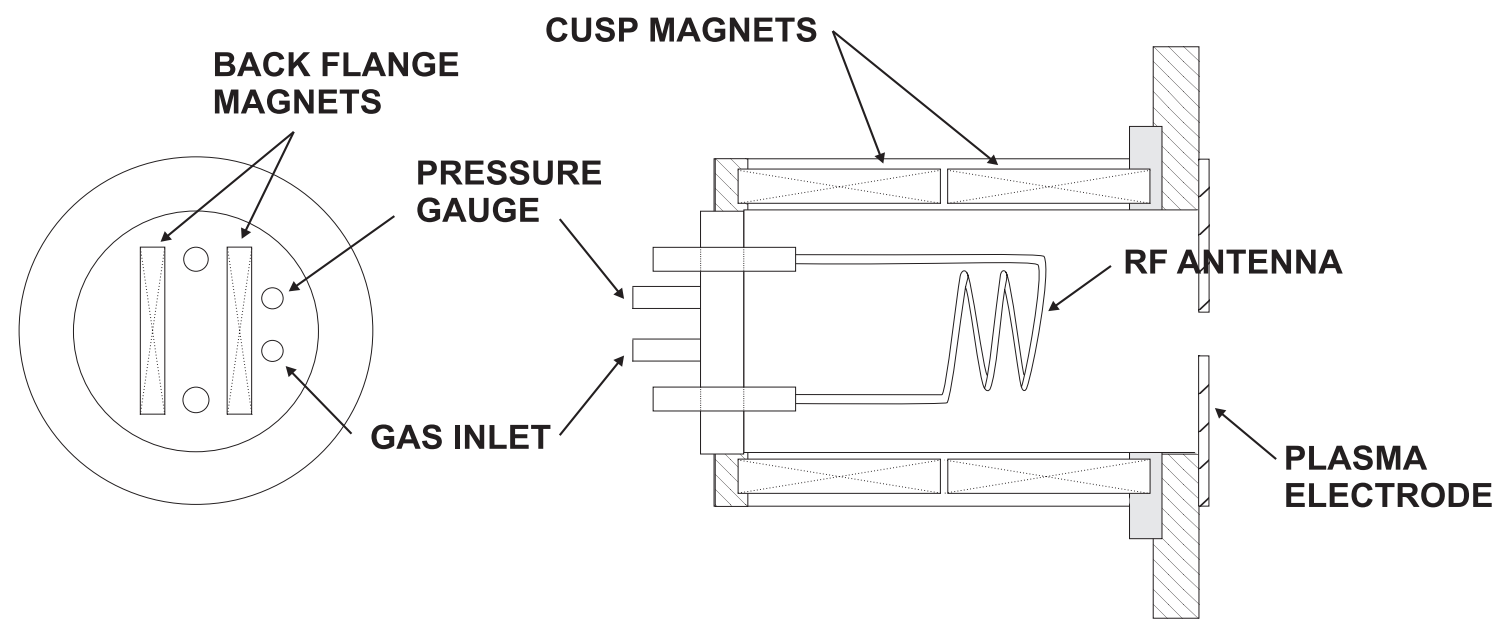

Figure 7.15: Schematic diagram of the RF-driven ion source.

finement is achieved by several arrangements of samarium cobalt rare-earth magnets. For confinement on the back flange and to protect the various feed-throughs from the plasma, a pair of permanent magnets, with opposite polarity facing the plasma chamber, are embedded in the back flange. A second, removable pair of magnets can be positioned inside the ion source, near the plasma electrode. Figures 7.16a and 7.16b show the ion source configurations with and without this set of magnets between the RF antenna and the plasma electrode. This front set of magnets with its opposite polarities facing the plasma, creates a transverse magnetic field across the discharge volume which serves two purposes. First, it provides ion and primary ionizing electron confinement on the plasma electrode side of the ion source. Second, this magnetic field has been shown to have a filtering effect. By providing confinement for the ionizing electrons, the transverse magnetic field effectively partitions the discharge volume into two regions, each with different electron temperatures. Upstream, toward the back flange of the ion source, is the ionization region, in which the ion- 


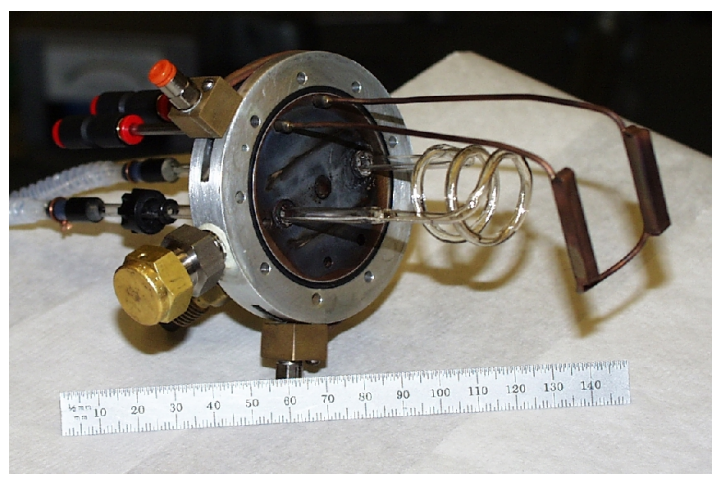

(a)

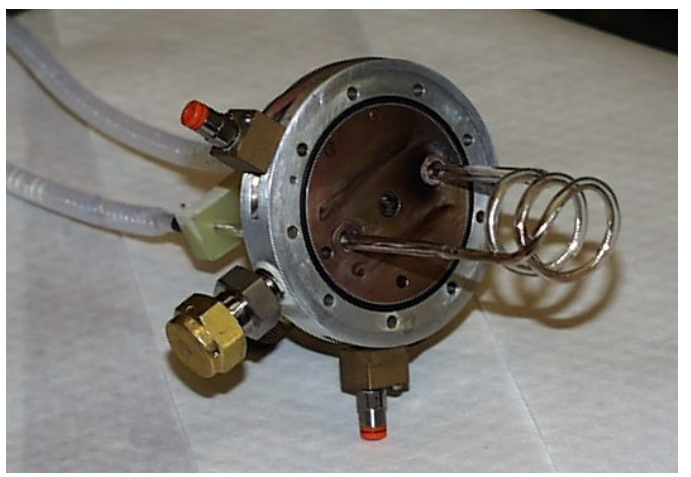

(b)

Figure 7.16: Ion source (a) with and (b) without magnetic filter.

izing primary electrons are generated and confined. This region is characterized by a higher background plasma electron temperature of $5 \mathrm{eV}$ or more. Downstream from the transverse field, toward the plasma electrode, is the extraction region, which is essentially free of ionizing electrons. This region, being shielded from energetic electrons by the transverse magnetic field, is characterized by a much lower electron temperature than the ionization region, typically less than $1 \mathrm{eV}$. As a result, the probability for ionization to take place in the extraction region is very small.

The permanent-magnet filter enables the formation of two plasmas with distinct electron temperatures, which for molecular gases, results in different regions containing different ion species. Experiments have shown that with hydrogen or deuterium gas, the monatomic species fraction can be enhanced with the addition of a filter field [53]. In light of this desirable effect with respect to neutron generation, the prototype ion source was designed to be fitted with removable filter magnets. The magnetic filter shown in Fig. 7.16a creates a transversal magnetic field of strength 130 Gauss at the midline between the two magnets. The vacuum seal on the magnetic filter legs was achieved with standard rubber O-rings. This feed-through design permits the position of the magnetic filter to be varied within the ion source.

The antenna is made up of two main components, the electric conductor and the insulator. The conductor responsible for the generation of the RF magnetic field inside the ion source is a water-cooled metallic wire. It is placed inside a quartz tube of $4.25-\mathrm{mm}$ diam in the shape of a coil, as shown in Figs. 7.16a and 7.16b. The coil outside diameter and length are $2.7 \mathrm{~cm}$ and $2.85 \mathrm{~cm}$, respectively. Being made of an insulating material, 
the tube floats at a low negative potential in the ion source. Therefore, bombardment and sputtering of the quartz tube occur only with low-energy particles, enhancing the lifetime of the antenna. The vacuum seal of the antenna is of the same type as the one for the magnetic filter, permitting longitudinal displacement of the antenna in the ion source. A $5 \mathrm{~kW}(\mathrm{cw})$, 13.56 $\mathrm{MHz}$ RF amplifier was used to power the ion source. Power is delivered to the ion source impedance matching network via a flexible coaxial cable of $50 \Omega$ nominal impedance. To reduce health hazards associated with radioactivity and neutron production, hydrogen is used instead of deuterium and tritium.

\subsubsection{Ion beam extraction system}

To characterize the ion source output, an extraction system consisting of two copper electrodes was attached to the ion source. The ion source and the two electrodes are shown in Fig. 7.17. The first electrode, or plasma electrode, contained a 2-mm-diam

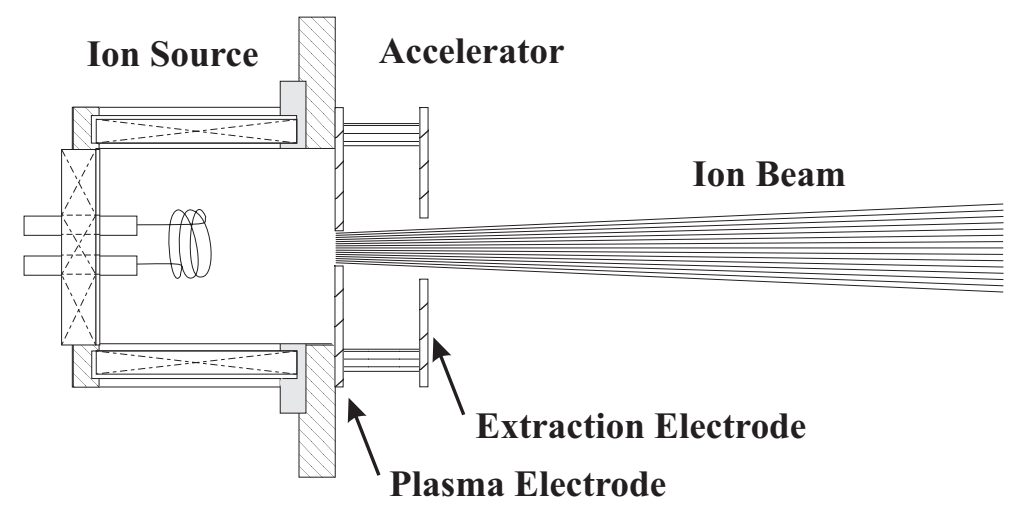

Figure 7.17: Ion beam extraction system.

aperture centered on the ion source axis. The second electrode - extraction electrode - had a 2-mm-diam aperture and was carefully aligned with the plasma electrode. The two electrodes were separated by a $2 \mathrm{~mm}$ gap. A variable 0 to $10 \mathrm{kV}$ high voltage power supply connected the ion source and the plasma electrode, while the extraction electrode was at ground potential. The extraction system was high-potted successfully up to $10 \mathrm{keV}$ at the vacuum chamber's base pressure, without high voltage breakdown across the gap. A heavy-duty, large matching network was employed for both hydrogen ion species and current measurements. Both ion source at high voltage installed on the accelerator column and the matching network are shown in Fig. 7.18. 


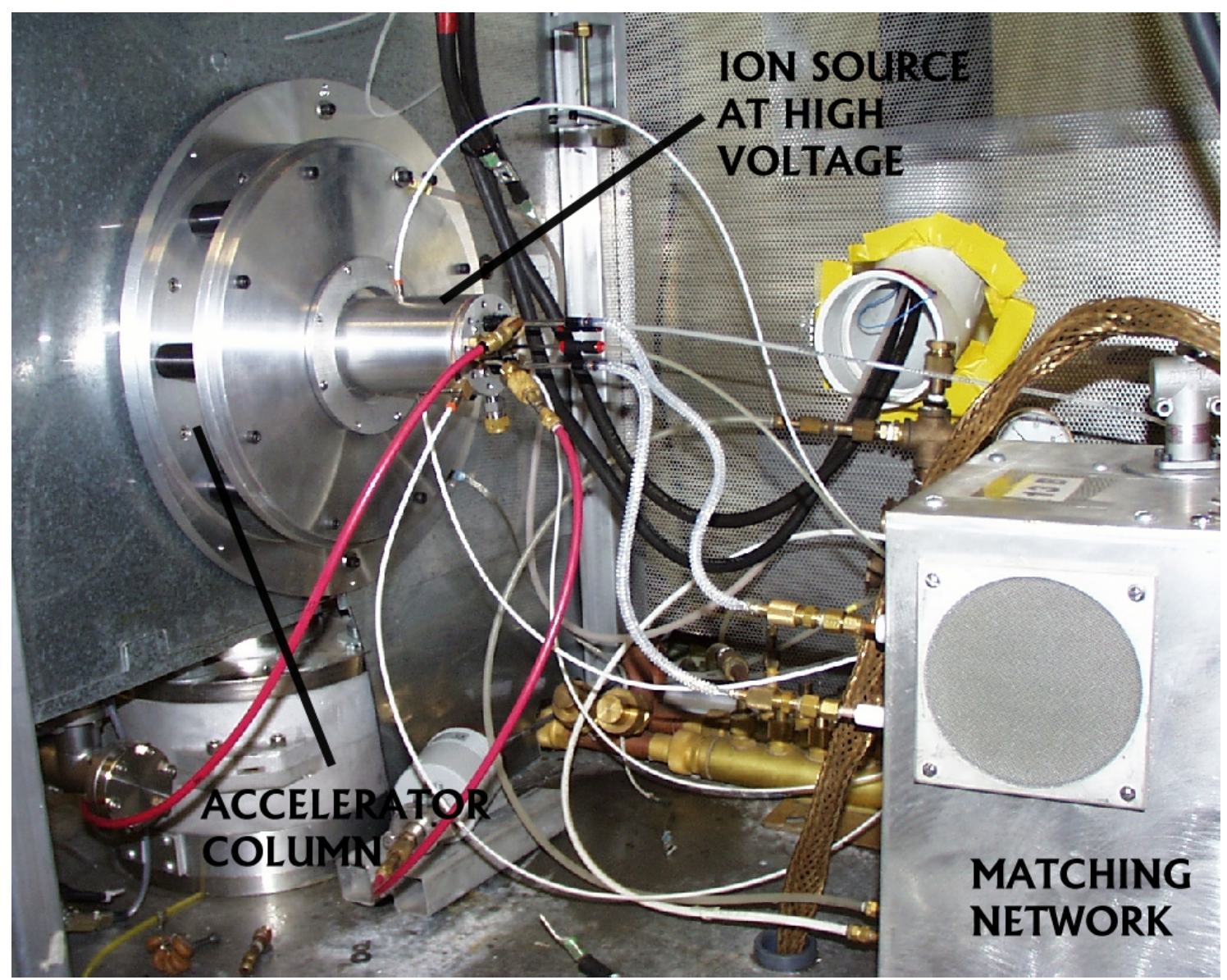

Figure 7.18: Matching network and ion source installed on the accelerator column.

\subsubsection{Ion species measurements}

To measure the ion species distribution in the ion source, we extract an ion beam out of the ion source and make use of a magnetic deflection mass spectrometer. Mass separation is achieved by Lorenz force of a magnetic field. When an ion beam enters a region of uniform magnetic field normal to the trajectory of the ion beam, the ions experience a bending force perpendicular to both the ion velocity $\vec{v}$ and the magnetic field $\vec{B}$, in a direction given by the vector product $\vec{v} \times \vec{B}$. The equation giving the force $\vec{F}$ exerted on an ion of charge $q$ and mass $m$ moving with velocity $\vec{v}$ in a magnetic field $\vec{B}$ is:

$$
\vec{F}=q \vec{v} \times \vec{B}
$$


where the norm of the velocity $\vec{v}$ is related to the ion energy by

$$
\begin{aligned}
E & =\frac{1}{2} m|\vec{v}|^{2} \\
& =q U
\end{aligned}
$$

where $U$ is the electrostatic accelerating voltage.

Using Newton's law, we can deduce the cyclotron radius of the ions of energy $E$ in the magnetic field:

$$
R \approx \frac{114}{B} \sqrt{\frac{U \cdot m}{q}}
$$

where $B$ is the magnetic field in Gauss, $R$ is the cyclotron radius in centimeters, $U$ is the extraction voltage in volts, $m$ is the ion mass in atomic mass units, and $q$ is the ion charge state. For a given extraction voltage $U$ and magnetic field $B$, the radius increases with the ion mass $m$.

\section{Experimental setup}

For the ion species measurements, a mass spectrometer as shown in Fig. 7.19 is located at the exit of the extraction electrode. Being located beyond the accelerating gap, the ion beam enters the mass spectrometer with a constant energy. The purpose of the narrow slit at the entrance of the mass spectrometer is to provide a ribbon beam with minimal divergence in the ion beam plane in order to improve the mass resolution of the spectrometer. Inside the mass spectrometer, a Faraday cup is positioned off the beam trajectory axis in the plane of the ion beam trajectory. The Faraday cup also has a narrow slitted aperture for the same reason as above. The magnetic field is generated in the mass spectrometer by an electromagnet and can be varied. Because of the magnetic field, ion beams with different ion masses, charge states and thus different cyclotron radii (see Eq. 7.7) are present in the mass spectrometer.

The trajectory of a particular ion is shown in Fig. 7.19. As the magnetic field increases, the different beam trajectories are gradually bent and intercepted by the Faraday cup. For ions with a given charge state, those with lower mass are first deflected into the cup. With increasing magnetic field strength, heavier ions are bent and collected in

the Faraday cup. The magnetic field is assumed to be proportional to the current flowing through the electromagnet coils. A plot of the Faraday cup current versus electromagnet current yields a spectrum giving the mass-to-charge composition of the beam. 


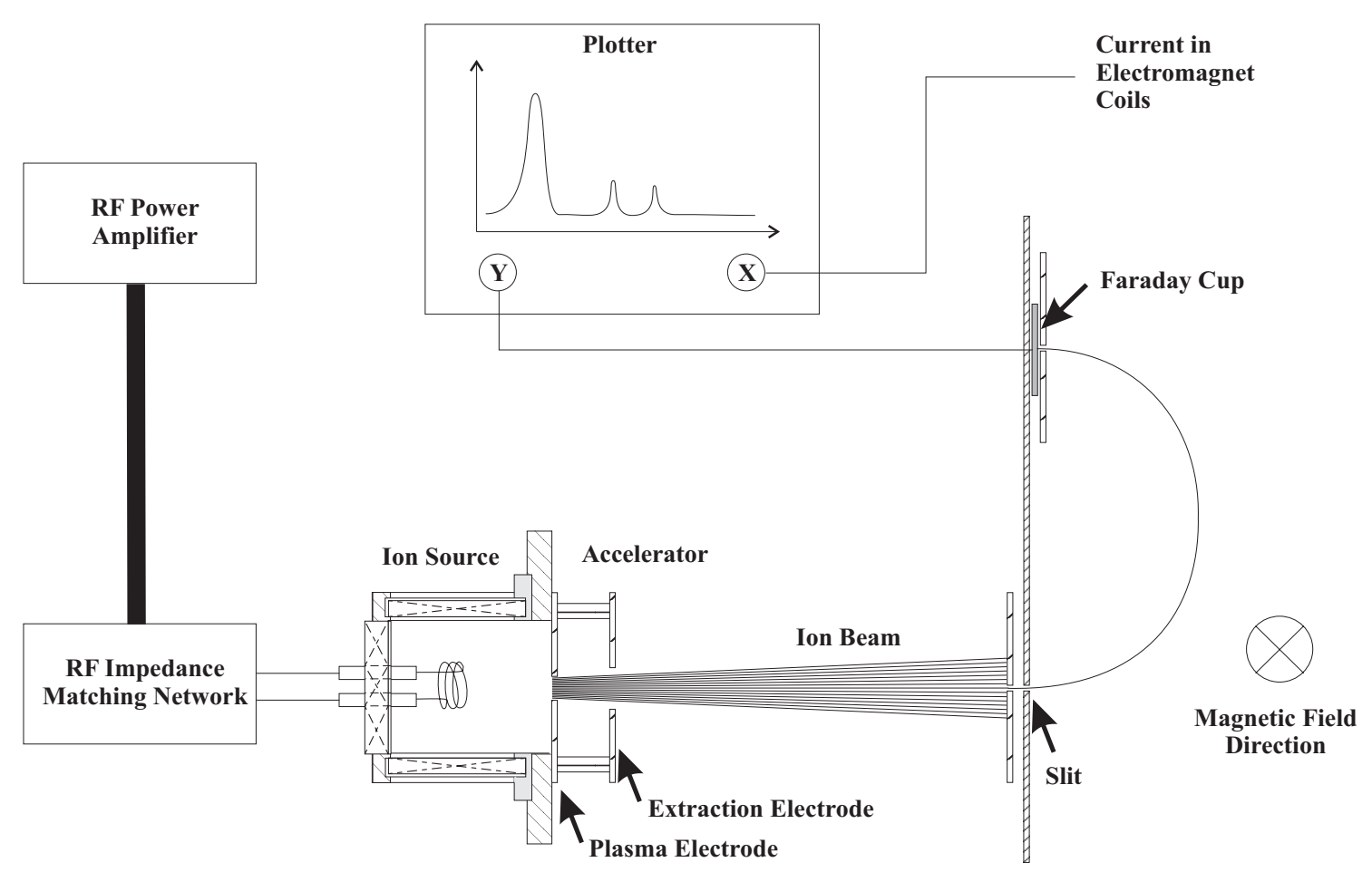

Figure 7.19: Experimental setup for the ion species measurements from the prototype ion source.

Figure 7.20 shows a sample spectrum obtained using the experimental setup described above. The operating conditions were a gas pressure of 7 mTorr, and a $\mathrm{cw}$ RF power of $1500 \mathrm{~W}$. The three first peaks correspond to the three hydrogen ion species $\mathrm{H}^{+}, \mathrm{H}_{2}^{+}$and $\mathrm{H}_{3}^{+}$. The sharpness of the peaks reflects the high mass resolution of the mass spectrometer. To compute the hydrogen ion species fractions in the spectrum, the peak heights are measured.

Several peaks corresponding to ions of higher masses are also visible in the spectrum. They are mostly due to ionized impurities coming off the walls of the ion source at early times when the walls are heated up. The longer the ion source runs, the fewer the impurities and the cleaner the spectrum. Impurities are highly undesirable in the context of the neutron production. Any impurity will contribute to the ion beam reaching the target and therefore compete against hydrogen isotope ions in the production of neutrons. This will have the adverse effect of reducing the neutron output. Additionally, the heavier mass impurities will result in significant sputtering of the target, which will eventually limit the lifetime of the target. In the final neutron tube, this issue will have to be addressed. A 


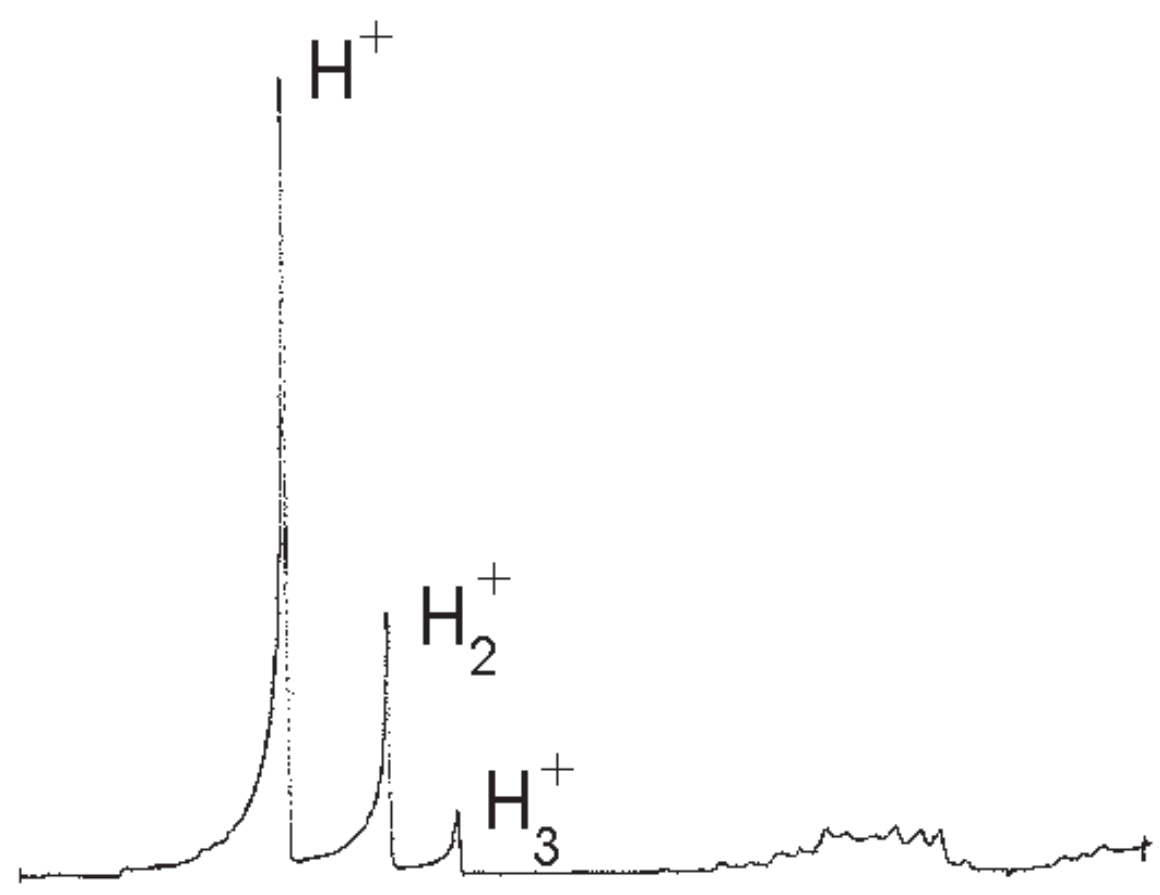

Figure 7.20: Sample ion species distribution measured with the mass spectrometer.

thorough baking procedure should be used to reduce outgassing during operation.

\section{Ion species distributions with and without magnetic filter}

The hydrogen ion species distributions were measured for different source pressures and RF power levels. The first set of experiments was done without magnetic filter. The coil of the RF antenna was centered with the axis of the ion source and is $4.0 \mathrm{~cm}$ away from the plasma electrode. Figure 7.21 shows the hydrogen ion species distribution as a function of the gas pressure for a fixed $\mathrm{cw}$ RF power of $1200 \mathrm{~W}$. The monatomic ion fraction is basically independent on the gas pressure over the pressure range covered by the experiment. The triatomic ion species fraction increases with the gas pressure at the expense of the diatomic ion species fraction. These two observations were made for several different $\mathrm{cw}$ RF powers.

In the next set of experiments, the ion species distributions were determined for the ion sources with and without magnetic filter as a function of the cw RF power. When the magnetic filter was located $1.7 \mathrm{~cm}$ from the plasma electrode and the coil of the RF antenna was pulled backwards to $5.0 \mathrm{~cm}$ from the plasma electrode. The results are plotted 


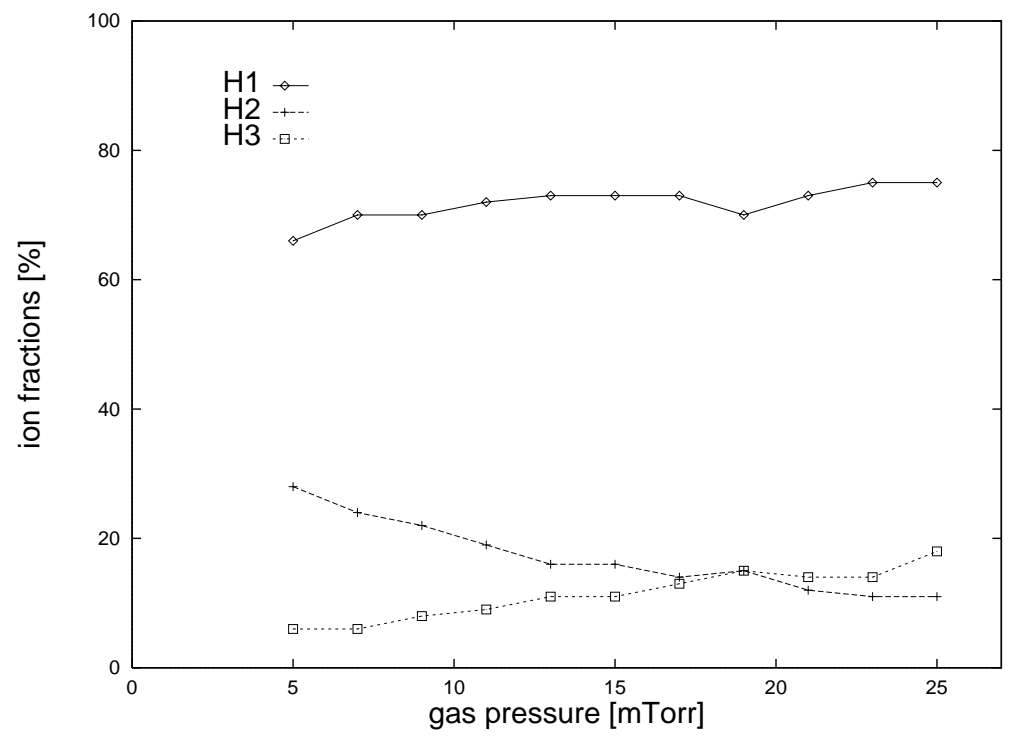

Figure 7.21: Hydrogen ion species fractions versus hydrogen gas pressure, $\mathrm{cw}$ RF power set to $1200 \mathrm{~W}$.

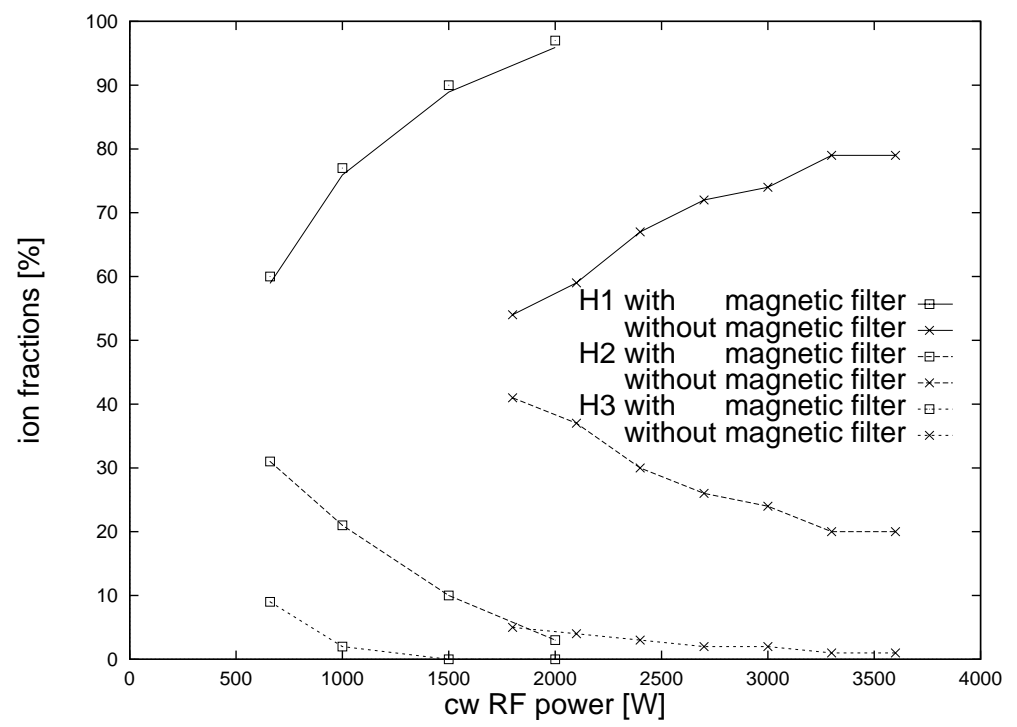

Figure 7.22: Hydrogen ion species fractions versus cw RF power for gas pressure of $4 \mathrm{mTorr}$, with and without magnetic filter.

in Fig. 7.22 for a hydrogen pressure of $4 \mathrm{~m}$ Torr. We observe that the monatomic ion species fraction is effectively enhanced by the magnetic filter in the source chamber. The filterequipped source produces a higher $\mathrm{H}^{+}$fraction than the source without magnetic filter for all power levels. In the best case, $97 \%$ of $\mathrm{H}^{+}$were obtained with the magnetic filter, to 
compare with $79 \%$ without magnetic filter. Figure 7.23 shows the spectrum measured by the mass spectrometer in this particular case.

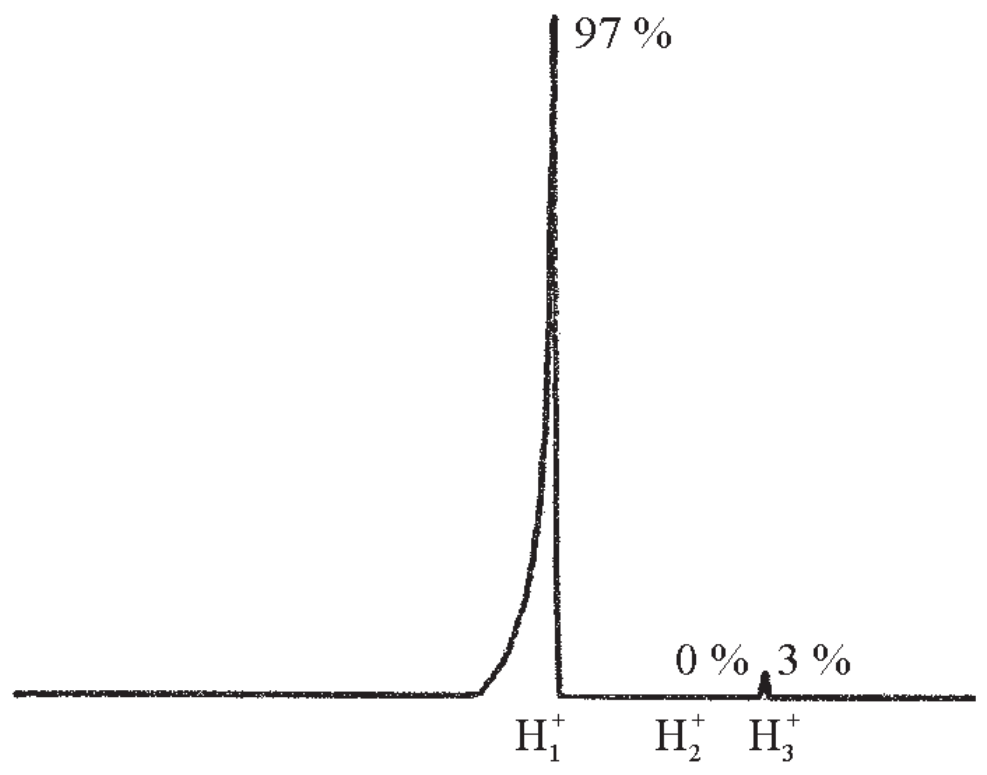

Figure 7.23: Hydrogen ion species distribution obtained with a cw RF power of $1200 \mathrm{~W}$ and a gas pressure of $4 \mathrm{~m}$ Torr in the filter-equipped ion source.

\section{Minimum pressure and RF power level required to sustain plasma}

In order (a) to avoid voltage breakdown in the accelerator column, (b) to decrease the ion beam losses by electron capture reaction with the neutral gas in the accelerator column, the gas pressure cannot exceed a few mTorrs. In addition to the monatomic hydrogen ion species enhancement, the use of a magnetic filter decreases the minimum gas pressure required to sustain the plasma at a given RF power level, as can be observed in Fig. 7.24, where the minimum cw RF power to sustain the plasma is plotted as a function of the gas pressure. The minimum gas pressure goes down from $4 \mathrm{mTorr}$ for the source without filter to $2 \mathrm{~m}$ Torr for the filter-equipped source. Also, lower RF power is needed to sustain the plasma with the magnetic filter: $660 \mathrm{~W}$ instead of $1800 \mathrm{~W}$ at 4 mTorr. 


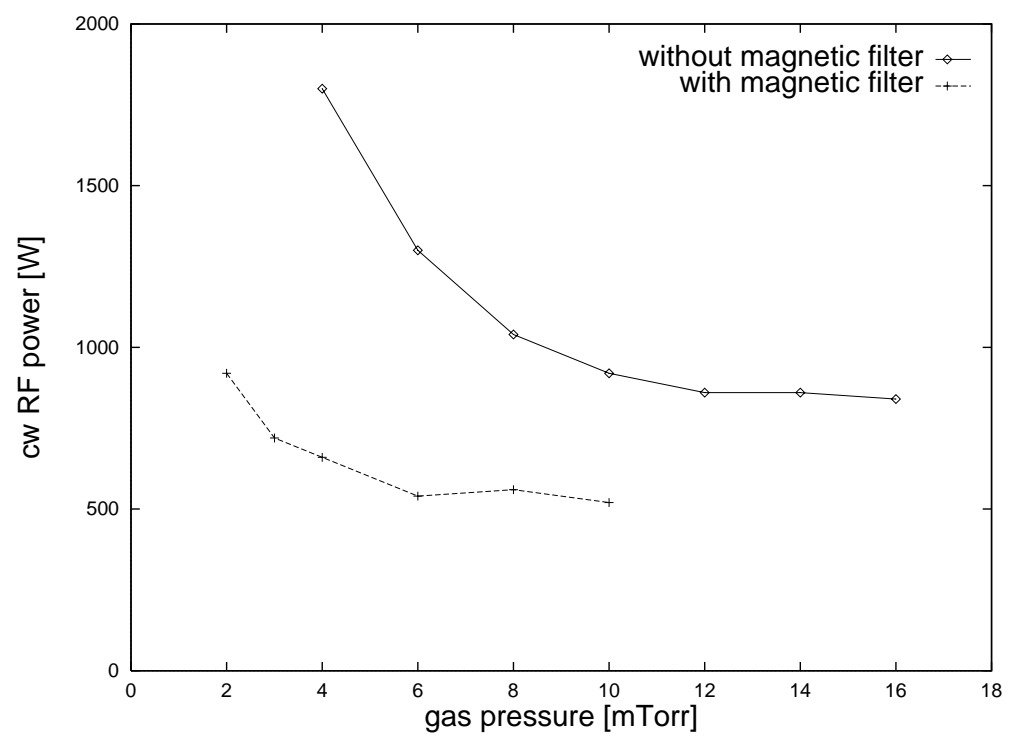

Figure 7.24: Minimum cw RF power to sustain plasma for different gas pressures.

\subsubsection{Ion beam current density measurements Experimental setup}

To measure the ion beam current density $J$, a Faraday cup assembly was positioned downstream from the extraction electrode aperture. The beam dump assembly, shown in Fig. 7.25 consisted of a graphite cup with permanent magnets placed in front of the collecting surfaces. The magnets are needed to prevent secondary emission electrons from leaving the graphite beam dump. Secondary electrons, when not suppressed, can lead to an overestimation of the beam current. In order to capture the entire beam, the whole assembly is placed as close as possible to the extraction electrode.

In this experiment, the Faraday cup was $2 \mathrm{~cm}$ away from the second electrode aperture. At the low vacuum chamber pressures during ion source operation, charge exchange in the drift region between the extraction electrode and the Faraday cup assembly will not significantly affect the beam current measurements. The Faraday cup assembly is electrically connected to a vacuum port via an electric wire. The collected current is then taken via a shielded coaxial cable to a current meter. Another electrical connection was made to the extraction electrode to measure the intercepted beam current. The Faraday cup assembly was mounted on a supporting structure that could be displaced transversally with a linear actuator. This system permitted the Faraday cup to be moved rapidly out 


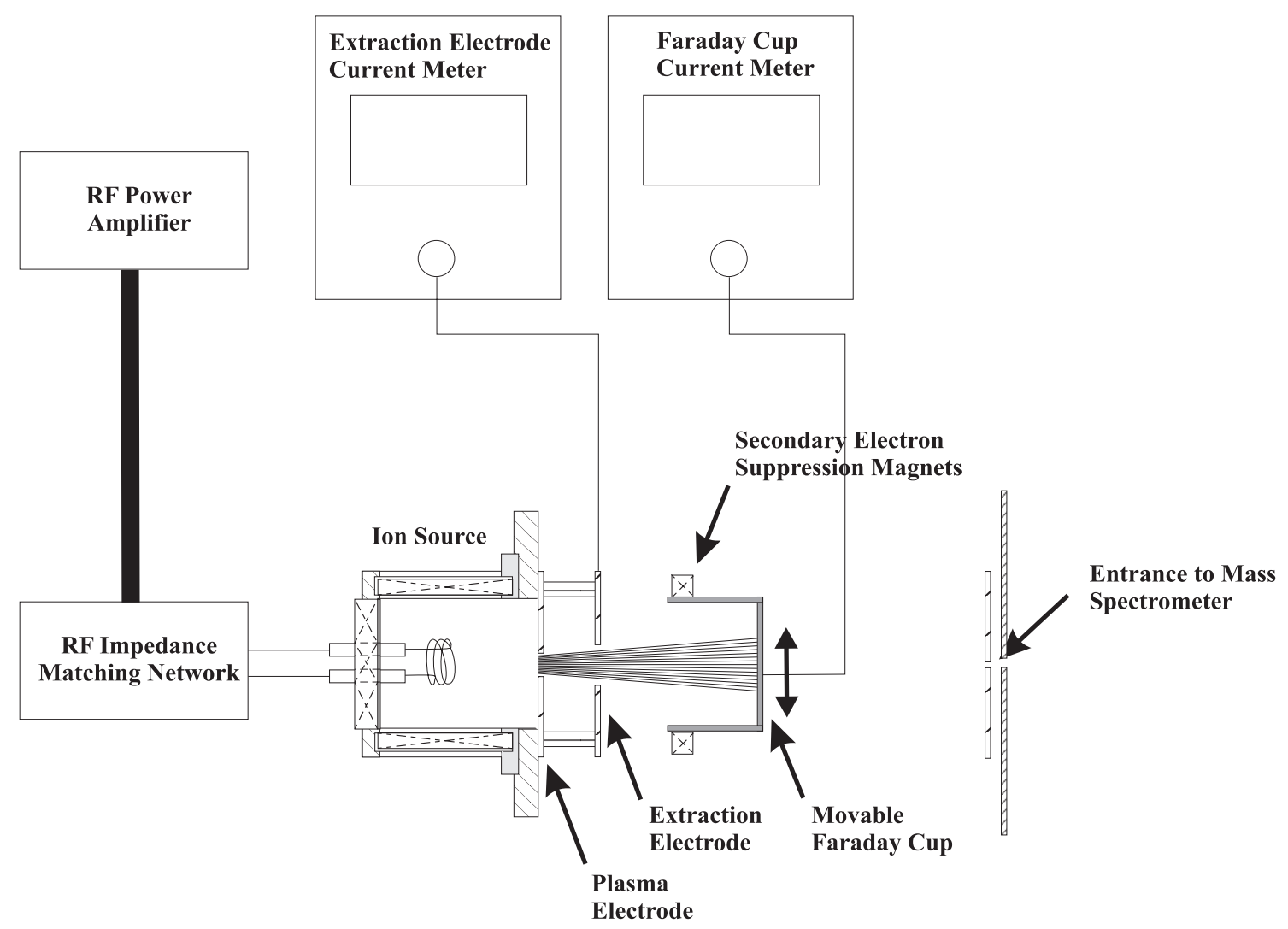

Figure 7.25: Schematic diagram of the experimental setup used for the current density measurement from the prototype ion source.

of the beam trajectory. The beam can then reach the entrance of the mass spectrometer unintercepted.

\section{Preliminary observations}

To determine the maximum extractable current from the ion source, the Faraday cup current density is plotted against the extraction voltage. The current density is simply obtained by dividing the ion beam current going through the plasma electrode by the area of the 2-mm-diam plasma electrode exit aperture. It is of interest to evaluate the current density rather than the current itself. This permits one to scale the ion source output current with the aperture area of the plasma electrode.

Figure 7.26 shows the current density as a function of the extraction voltage for four different RF power levels and at a gas pressure of 4 mTorr. For these experiments, the magnetic filter and antenna coil were positioned at 1.7 and $5.7 \mathrm{~cm}$, respectively, from the 


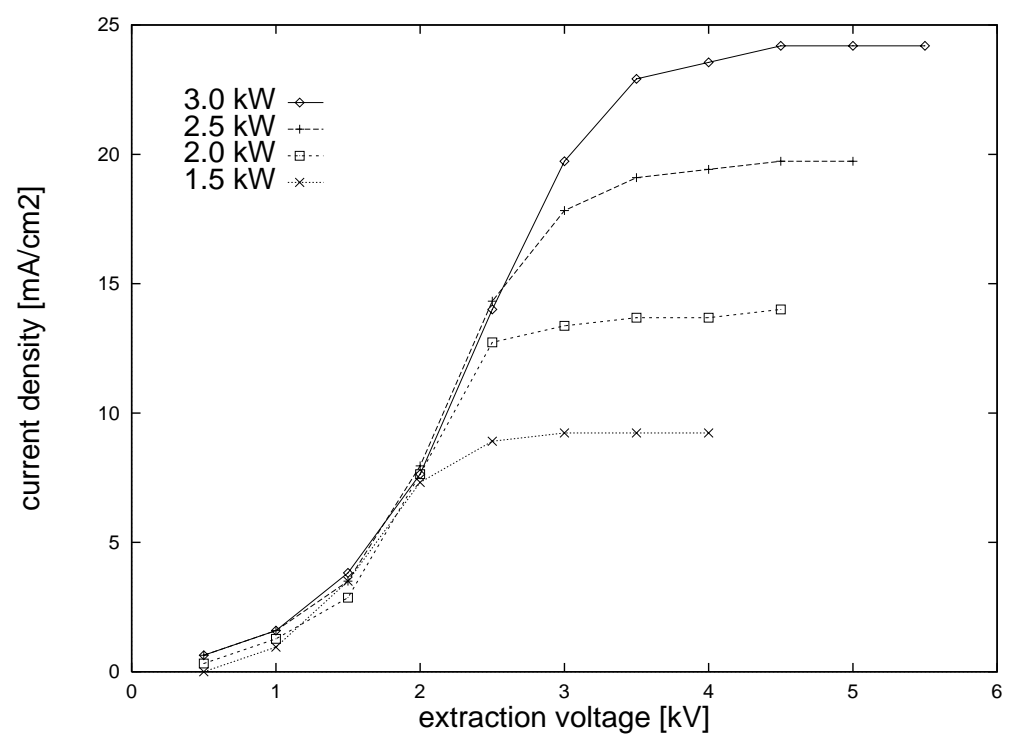

Figure 7.26: Current density versus extraction voltage for different $\mathrm{cw}$ RF power levels for a filter-equipped ion source and a gas pressure of 4 mTorr.

plasma electrode. All traces increase steeply at low extractions voltages and reach a platean at higher voltages. They share an identical rising slope at lower extraction voltages. The traces separate from this common slope only at higher voltages. The traces at lower RF power reach a plateau with lower values, while the traces at higher RF power reach higher plateau values.

Two regimes can be identified. At lower extraction voltages, the extracted current for all RF power levels increase steeply with the extraction voltage. This corresponds to a voltage-limited extraction regime, where the extracted current is set by the available extraction voltage. As the extraction voltage is further increased, the rate of increase in extracted current decreases and eventually saturates. This corresponds to an emission-limited regime, in which the extraction of all of the available current is reached. By measuring the current level of the emission-limited regime, the true maximum extractable current density for the ion source can be determined, as stated in the Child-Langmuir-Schottky law, see Sec. 7.4.1. In our experiment, the maximum extractable current density at a given gas pressure and cw RF power level was taken equal to the height of the platean of each trace.

Sometimes, a slight increase in the current density is observed with increasing extraction voltages. Figure 7.27 shows an example of this behavior. This is attributed to an increase in the effective ion collection area, which now extends beyond the aperture area, 


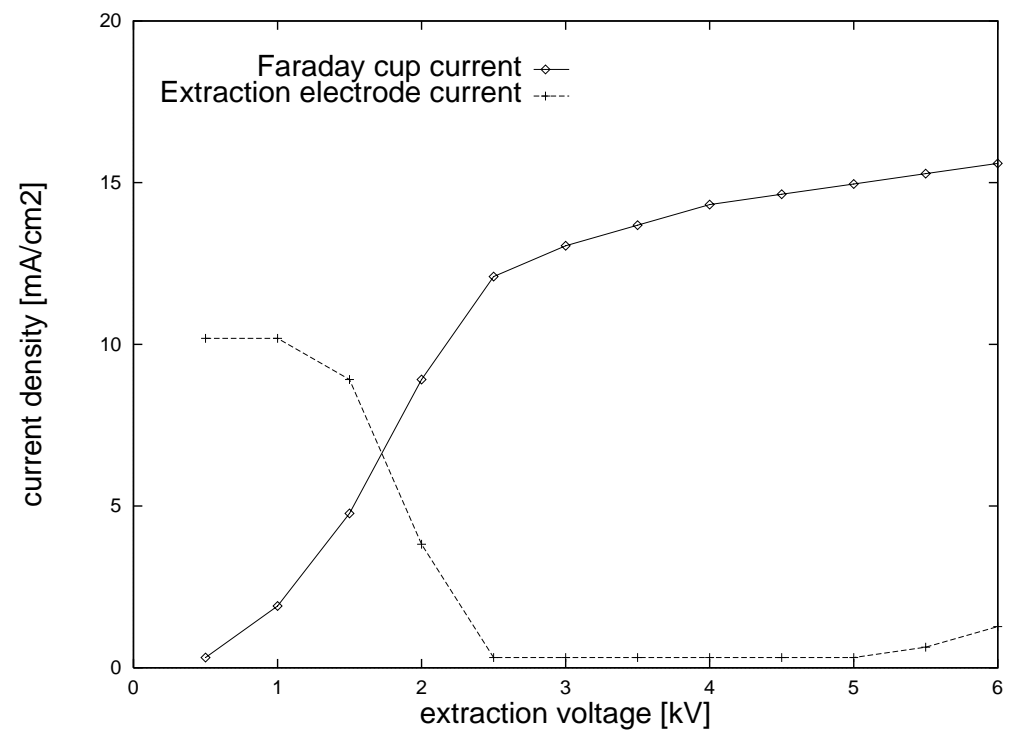

Figure 7.27: Faraday cup and extraction electrode current versus extraction voltage for a filter-equipped ion source, an RF power of $1.5 \mathrm{~kW}$ and a gas pressure of $8 \mathrm{~m}$ Torr.

into the ion source. At the higher extraction voltages, the plasma becomes under-dense and the extraction field penetrates beyond the aperture into the ion source, see Sec. 7.4.1. This electric field distorts the plasma sheath near the aperture, increasing the surface area across which the ions are collected for extraction.

At low extraction voltages, the current intercepted by the extraction electode could be as high as $2 / 3$ of the maximum extractable current measured in the Faraday cup, it decreased very rapidly as the extraction voltage was raised. This is shown in Fig. 7.27. A fraction of this current is from the accelerated ion beam being intercepted by the extraction electrode. The remaining fraction can be attributed to secondary electrons emitted from the extraction electrode surface as a result of energetic ion collisions. The secondary electrons leave the extraction electrode surface, accelerate toward the plasma electrode under the influence of the extraction field, and contribute to the net current measured at the extraction electrode. The yield of the secondary emission electrons from a given material surface depends on the ion energy and mass. Because of the difficulty in discriminating between ion current and secondary emission electron current, the total extracted current was assumed to be equal to the Faraday cup current only. This assumption underestimates the actual ion source output.

At high extraction voltages, the current on the extraction electrode was negligible. 
In terms of ion beam optics, the ion beam is intercepted by the extraction electrode at low extraction voltages because the electric extraction field is too weak to focus the beam within the extraction electrode aperture. As the extraction voltage increases, the beam is more focused by the stronger electric field and is no longer intercepted by the extraction electrode. This explanation is in good agreement with the experimental results.

Overfocussing of the beam can also occur when the electric field between the plasma electrode and extraction electrode is too large. The ion beam then crosses over between the plasma electrode and the extraction electrode and part of it is intercepted by the extraction electrode. Overfocussing was sometimes observed in our experiments at extraction voltages greater than $5.5 \mathrm{kV}$, as shown in Fig. 7.27. In this regime, the extraction electrode current increases with the extraction voltage as a larger fraction of the ion beam is intercepted by this electrode. Since overfocussing occured at extraction voltages well beyond the emission-limited regime, the ion beam optics were still adequate in this regime and the the maximum extractable current was still determined correctly in our experiments.

\section{Experimental results}

The extractable current measurements were taken for different source pressures and RF power levels, with and without magnetic filter. For the experiment without magnetic filter, the antenna coil was $4.7 \mathrm{~cm}$ from the plasma electrode. For the experiments with the magnetic filter, the filter was positioned $1.7 \mathrm{~cm}$ away from the plasma electrode. Two sets of experiments were performed in which the antenna coil position was changed from 5.0 to $5.7 \mathrm{~cm}$ away from the plasma electrode.

Figure 7.28 shows the extracted current density at saturation versus RF power for the ion source without magnetic filter. The current density increases linearly with the RF power. It also increases with the gas pressure in the ion source.

It has been shown that the presence of a magnetic filter can enhance the monatomic species fraction output [53]. Therefore, the current density performance of the ion source with magnetic filter was investigated. Figures 7.29 and 7.30 show the extracted current density at saturation versus RF power for three different gas pressures and two different RF antenna coil positions. The ion source output was found to be reduced by up to $50 \%$ with the magnetic filter. This reduction in current is the result of the transverse magnetic field, which enhances plasma confinement and inhibits ion flow to the extraction aperture. 


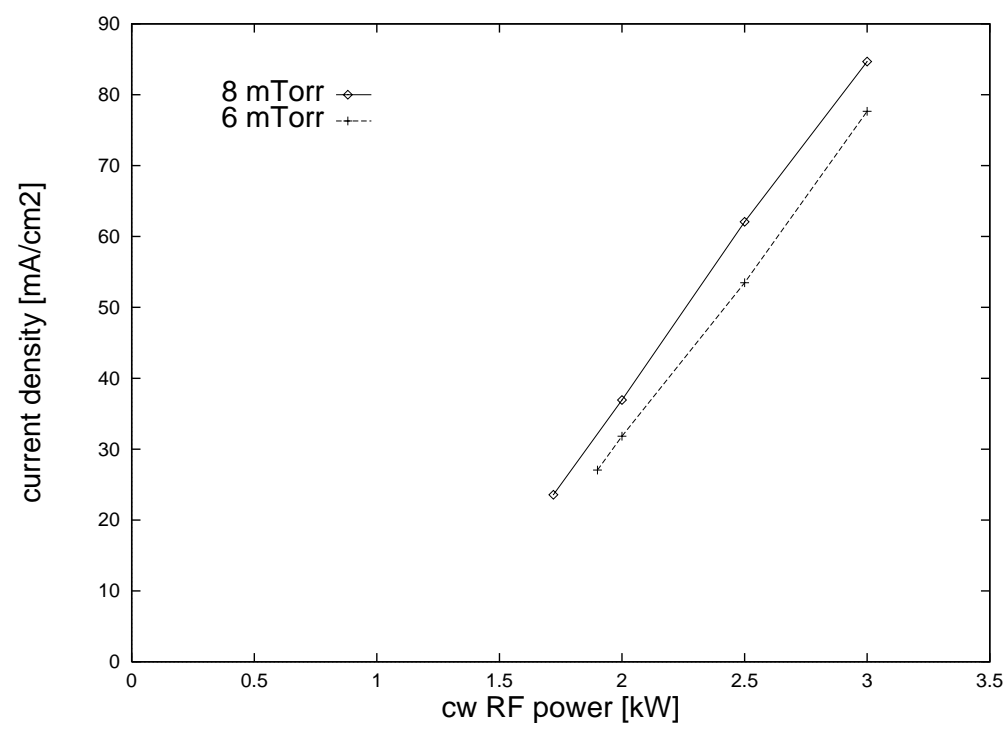

Figure 7.28: Extracted current density at saturation versus cw RF power for the ion source without magnetic filter for two gas pressures.

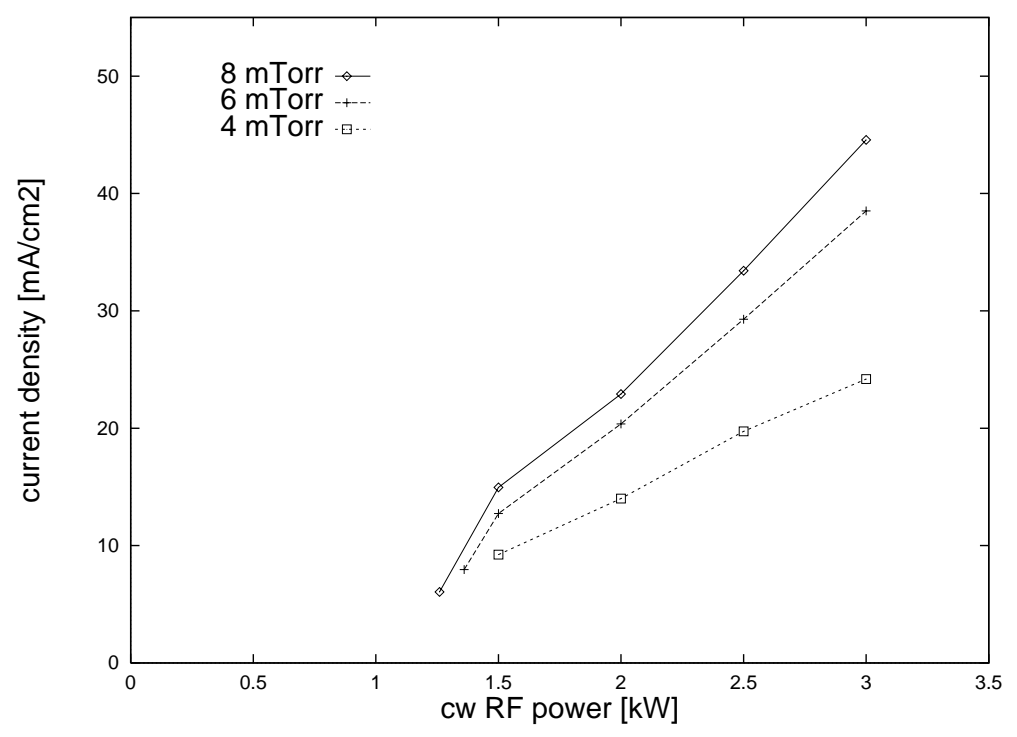

Figure 7.29: Extracted current density at saturation versus RF power for the filter-equipped ion source with antenna coil positioned $5.7 \mathrm{~cm}$ away from the plasma electrode.

\subsubsection{Summary of results}

A prototype 5-cm-diam RF-driven multicusp ion source was fabricated and used to generate plasmas. Two different magnetic filter configurations were compared in terms of ion species distribution and extractable current density. The first configuration had a 


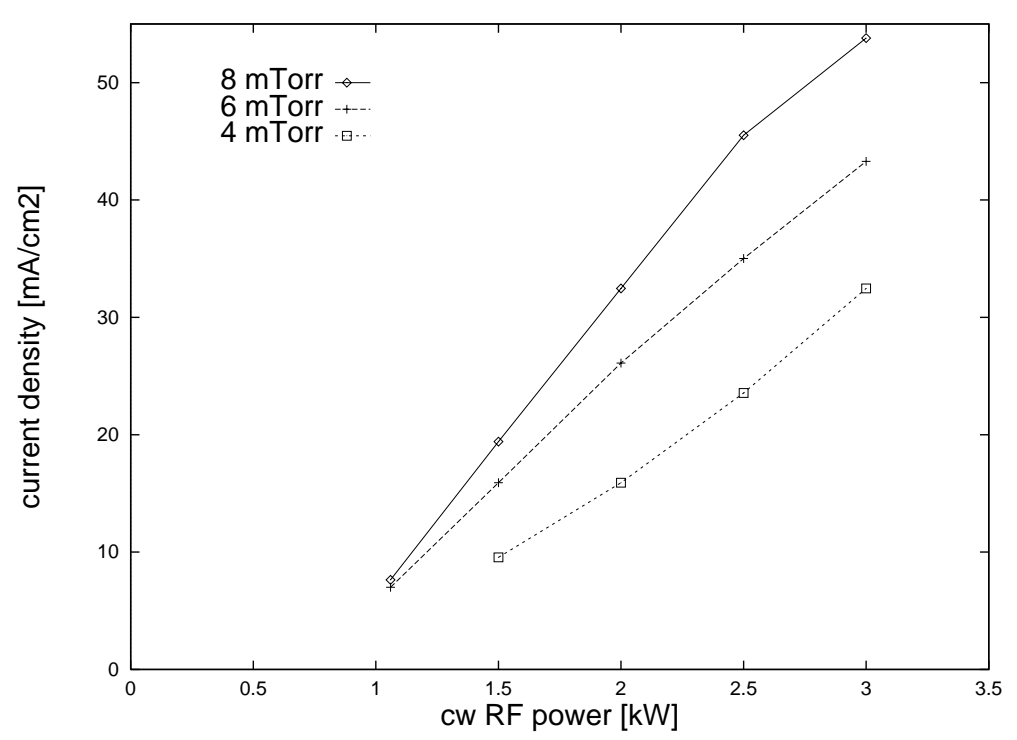

Figure 7.30: Extracted current density at saturation versus RF power for the filter-equipped ion source with antenna coil positioned $5.0 \mathrm{~cm}$ away from the plasma electrode.

magnetic filter located near the plasma electrode to confine the primary ionizing electrons, while the second had no filter. With a hydrogen discharge, higher monatomic fractions were obtained with the filter-equipped ion source. Monatomic fractions as high as $95 \%$ had been achieved with this configuration. In terms of extractable ion beam current density, the filter-equipped ion source gave lower current densities than the ion source without the magnetic filter.

In addition to achieving a high monatomic ion species fraction, the filter-equipped ion source has another advantage, it can be operated at lower gas pressures ( 1 to 2 mTorr) than the other ion source. Since the accelerator column is operating at the same gas pressure - a few mTorrs - as the ion source in a sealed neutron generator, operation of the ion source at low gas pressure is necessary to reduce both high-voltage breakdown and charge exchange in the accelerator column. Indeed, charge exchange processes would result in beam losses.

\subsection{Accelerator column}

The purpose of the accelerator column is to extract charge particles from the ion source and to form a useful beam, which then impinges onto a target. Extraction is 
performed by applying a potential difference between a source of charged particles and a region to which they have to be transported. Positive ions are extracted through an aperture in the plasma electrode. Given the extraction electrode is at a lower potential than the plasma electrode, positive ions are attracted by the negative electrostatic potential induced by the extraction electrode and form an ion beam.

In the case of the neutron tube, deuterons or tritons are accelerated onto a target with an energy of 100 to $200 \mathrm{keV}$, where fusion reactions occur with deuterium and tritium occluded in the target. The quantities of interest for the neutron tube are the total ion beam current, the trajectory of the ion beam, and the area of the beam on the target. The total beam current dictates the neutron yield. The second quantity is important for the design of the accelerator column. The electrodes must not obstruct the path of the ion beam to avoid electrode sputtering and secondary electron emission, which when accelerated to 100 to 200 $\mathrm{keV}$ could severely damage the plasma electrode and the ion source. The third quantity dictates the target lifetime, which is limited by sputtering of the thin titanium/scandium layer on the copper substrate. Sputtering is proportional to the current density on the target, which is obtained by dividing the ion beam current by the area of the beam on the target.

\subsubsection{Plasma extraction region}

The trajectories of the accelerated ions are affected by the strength of the extraction field and the shape of the ion emitting surface, commonly referred to as the plasma "meniscus". The meniscus corresponds to the boundary between the plasma and the ion beam. Its shape is determined by the electric field in the region of the aperture as well as the density and mobility of the charges in the plasma, i.e., the plasma temperature. The profile of the plasma meniscus is of great importance to the propagation of the ion beam. The plasma boundary moves so as to satisfy, to a first order, the Child-Langmuir-Schottky space charge law [54], with the ions emitted perpendicularly to the meniscus surface. This law gives the maximum current density that can be extracted between two planar electrodes, one of which is an emitting source of charges. The maximum current density that can be transported occurs under space-charge limited conditions and is given by:

$$
J=\frac{4}{9} \epsilon_{0} \sqrt{\frac{2 q}{m_{i}}} \frac{U^{3 / 2}}{d^{2}} \quad\left[A / m^{2}\right]
$$


where $J$ is the ion beam current density in ampers per square meters, $\epsilon_{0}$ is the permittivity of free-space $\left(\epsilon_{0} \approx 8.8 \times 10^{-12} \mathrm{~F} / \mathrm{m}\right), q$ and $m$ are the ion charge and mass in coulombs and kilograms respectively, and $U$ and $d$ are the diode extraction system's accelerating potential in volts and electrode separation in meters. When this equation is satisfied, we obtain a flat meniscus, which corresponds to the planar source of charges. If the left-hand side of the equation is smaller than the right-hand side, the ion extraction occurs in an emission-limited regime, in which the extraction field can remove more ions than are available.

The three general shapes for the meniscus are shown in Figs. 7.31. For an under-

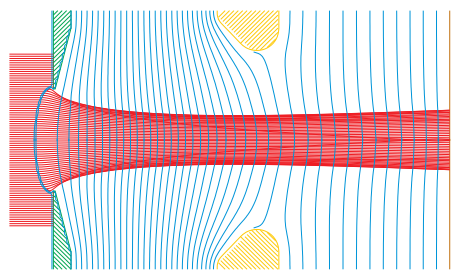

(a)

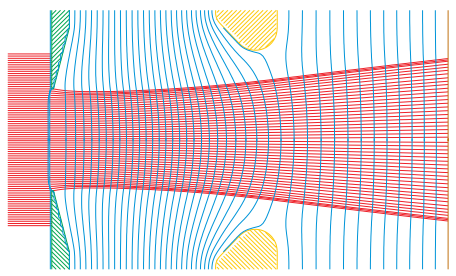

(b)

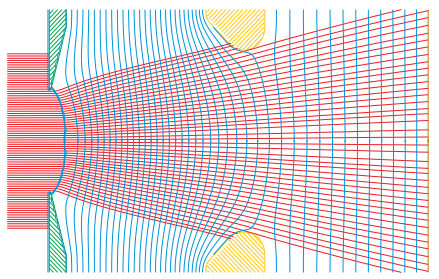

(c)

Figure 7.31: Menisci corresponding to (a) under-dense, (b) intermediate, and (c) over-dense plasma.

dense plasma, the meniscus is concave in shape, due to the strong penetration of the electric field induced by the extraction electrode inside the ion source. As a result, the ion beam converges due to the focusing effect of the meniscus. Under-dense plasmas were observed in Fig. 7.27, Sec. 7.3.4 for large extraction voltages. For the neutron tube, an under-dense plasma might cause problems due to the higher current densities on the target, which can limit its lifetime.

In the case of an over-dense plasma, the meniscus is convex, bulging outwardly from the ion source into the extraction gap. In this case, the beam is divergent and it impinges on the extraction electrode. This meniscus shape will result in a loss of ion beam current to the extraction electrode - as observed in Fig. 7.27. In addition, problems with sputtering and secondary electron emission will arise. In the ideal case of intermediate density, the meniscus is flat. This case corresponds to the equality in Eq. 7.8. The current density and extracting field are balanced on the meniscus, and an ion beam can be extracted and transported with the beam trajectories parallel to the beam axis.

In addition to the plasma and extraction electrodes, another electrode is placed between the extraction electrode and the target. Its role is to prevent the secondary emis- 
sion electrons emitted from the target by ion bombardment from streaming back to the accelerator electrodes and ion source with energies of 100 to $200 \mathrm{keV}$. This will prolong the lifetime of the ion source.

\subsubsection{Prototype accelerator column design}

A prototype accelerator able to deliver $1 \mathrm{~mA} \mathrm{cw}$ at 150 to $200 \mathrm{keV}$ has been designed. For the ion beam transport simulation, the ion beam is assumed to be a pure monatomic hydrogen ion beam and has a current density of $36 \mathrm{~mA} / \mathrm{cm}^{2}$ at the plasma electrode. This current density is within the current density ranges measured in Sec. 7.3.4. With an exit circular aperture of 2-mm-diam, this current density results in a total beam current of $1.1 \mathrm{~mA}$.

\section{Ion beam optics simulation}

An ion beam optics simulation code was used to design the prototype accelerator

column. IGUN (C) [55] is a two-dimensional code that computes the ion beam trajectory from the given accelerator geometry and the plasma conditions. Accelerator geometry includes electrode shapes, electrode potentials and relative positions. Plasma conditions include ion mass, electron and ion temperatures in the plasma, and the ion current density. IGUN computes the electric field in the accelerator column without the ion beam using Laplace's equation. The ion beam is then introduced in the accelerator. The electric field is then computed using Poisson's equation. The process is iterative. The beam trajectory and electric field calculations converge asymptotically to a solution. The two-dimensional calculations can be carried out in either cylindrical or slab geometries. After convergence, the code computes the equipotential line densities as a function of the longitudinal position in the accelerator column. This is useful when one has to determine the electric field strength at any location in the accelerator column.

The prototype accelerator column is not designed for sealed operation. An external pump will be used on the accelerator column side. Therefore, the gas pressure in the accelerator column will be much lower than in the ion source. The minimum inter-electrode spacing is determined by imposing a maximum potential gradient of $40 \mathrm{kV} / \mathrm{cm}$. Considering the breakdown limit given by the Paschen curves, this value $40 \mathrm{kV} / \mathrm{cm}$ is conservative. In order to suppress secondary electrons emitted from the target, the potential of the last or 
suppressor electrode was set at $10 \mathrm{kV}$ more negative than the target potential.

The field strength and equipotential line distribution provided by IGUN at the end of each run was useful in the design of the accelerator column. It provides us the information (a) that the maximum electric field strength was below the breakdown value and (b) that the secondary emission electrons produced by the target would effectively be suppressed. In order to satisfy this requirement an equipotential line more negative than the target potential by at least $\sim 200 \mathrm{~V}$ should cross the entire ion beam between the suppressor electrode and the target.

The accelerator column was designed to operate over a wide range of current densities, from $10 \mathrm{~mA} / \mathrm{cm}^{2}$ up to $100 \mathrm{~mA} / \mathrm{cm}^{2}$. This permits the ion source to operate at different RF power levels and gas pressures.

Another criterion for the design of the accelerator column was the beam size at the target. The beam size on the target was chosen such as to limit the power density on the target to $\sim 5 \mathrm{~mA} / \mathrm{cm}^{2}$, or $\sim 1 \mathrm{~kW} / \mathrm{cm}^{2}$ at $200 \mathrm{kV}$. This low power density is expected to provide long target lifetime. With a beam current of $\sim 1 \mathrm{~mA}$ and energy of $200 \mathrm{keV}$, a beam spot area on the target greater than $20 \mathrm{~mm}^{2}$ is necessary to stay under the power density limit. Since the beam area at the plasma electrode is $3.14 \mathrm{~mm}^{2}$, this means an expansion of the beam area by a factor $\sim 6$ between plasma electrode and target.

A single hole accelerator column was designed. Figure 7.32 shows the beam tra-

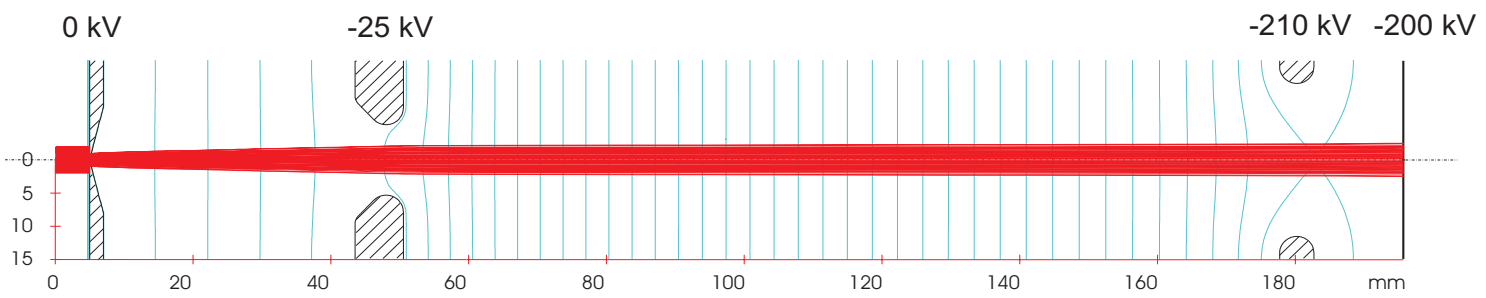

Figure 7.32: Single hole type accelerator column design using IGUN simulation code for proton beam. The current density at the ion source is $36 \mathrm{~mA} / \mathrm{cm}^{2}$.

jectory and equipotential line distribution for the triode configuration. The beam spot area at the target is $\sim 20 \mathrm{~mm}^{2}$. The same accelerator geometry could be used for a wide range of current densities by adjusting the extraction electrode potential. The higher the current density, the higher the potential difference between the plasma electrode and the extraction electrode. The current density was limited by (a) the voltage breakdown limit of $40 \mathrm{kV} / \mathrm{cm}$ in the gap between plasma and extraction electrodes and (b) the beam size that fits within 
the 23.2-mm-diam aperture of the suppressor electrode. The highest current density that the accelerator geometry could handle was $389 \mathrm{~mA} / \mathrm{cm}^{2}$. The beam trajectory and equipotential line distribution for this case are shown in Fig.7.33. The total beam current is 12.2

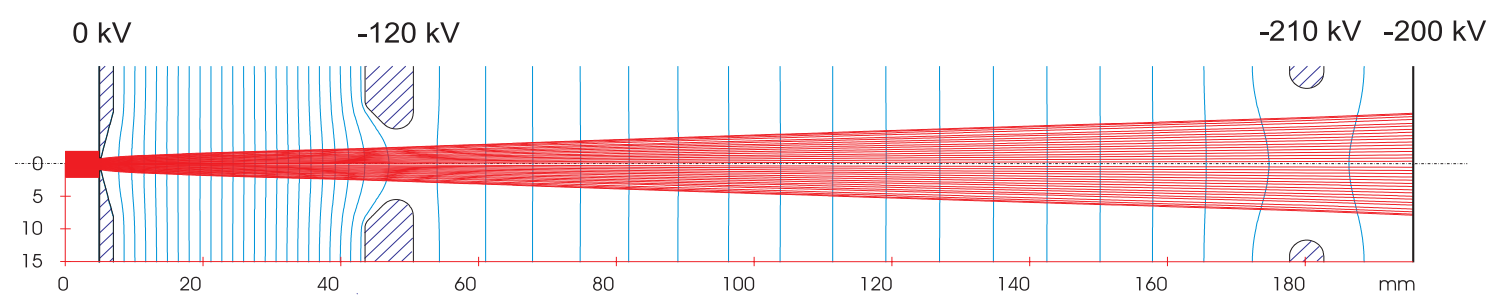

Figure 7.33: Single hole type accelerator column design using IGUN simulation code for proton beam. The current density at the ion source is $389 \mathrm{~mA} / \mathrm{cm}^{2}$.

$\mathrm{mA}$ and the beam spot area on target is $191 \mathrm{~mm}^{2}$. This results in a power density on the target slightly higher than the nominal $1 \mathrm{~kW} / \mathrm{cm}^{2}$.

\section{Accelerator column fabrication}

A large accelerator column was fabricated to study the hydrogen ion beam extraction and acceleration. Figure 7.34 shows a mechanical drawing of the accelerator column.

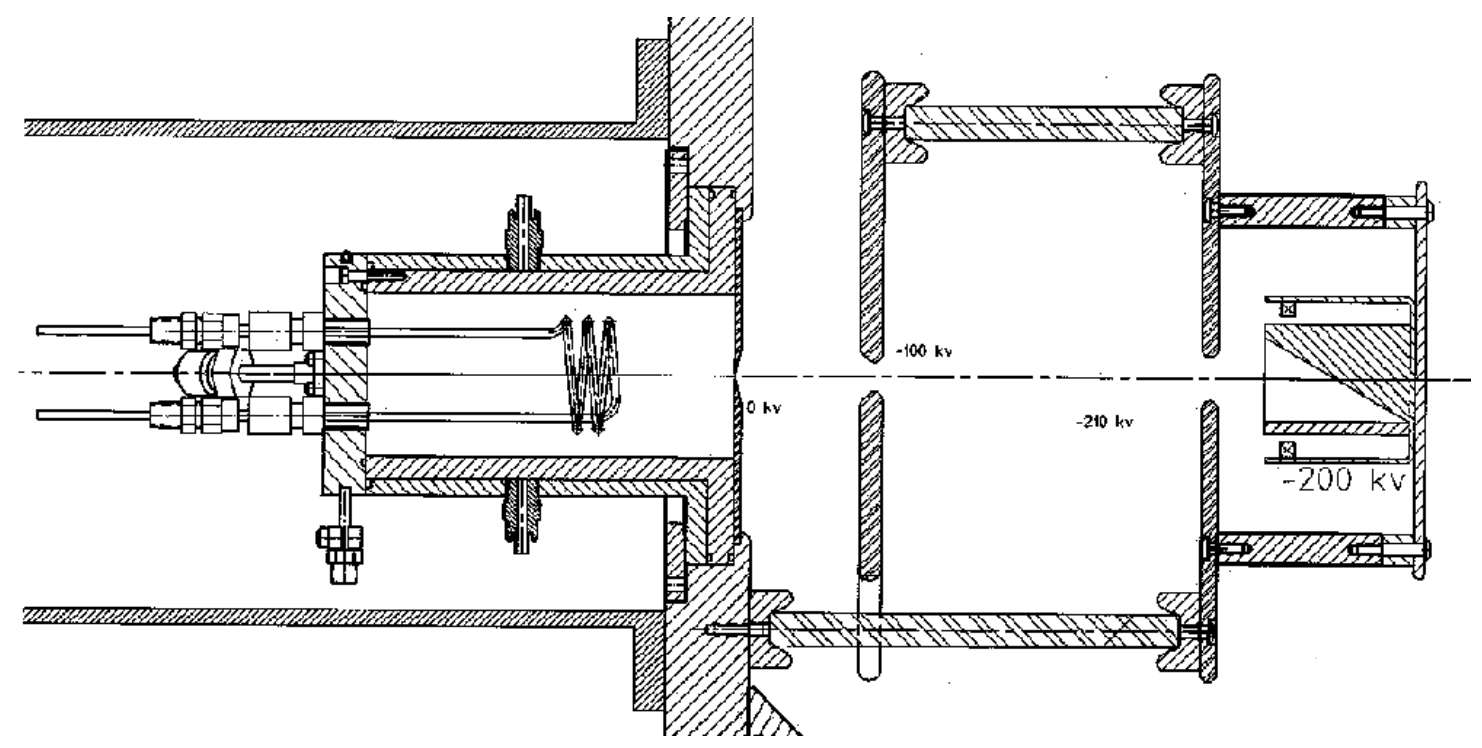

Figure 7.34: Mechanical drawing of the ion source and accelerator column.

The ion source and accelerator column were mounted on the opposite sides of a large flange 
referred to as the main flange further in the text. The main flange was closing off a large vacuum chamber on the accelerator column side. The shapes of the plasma electrode and extraction electrode apertures were designed with care, since they have a strong effect on the plasma meniscus and the resulting beam trajectory. To simplify fabrication, all the electrodes were flat and made out of copper. As shown in Fig. 7.34, the suppressor electrode was mounted on the main flange of the vacuum chamber using ceramic rods for insulation.

Because of the small distance between the plasma electrode and the extraction electrode, and the large potential difference between them, the extraction electrode could not be mounted directly on the main flange like the suppressor electrode. Instead, it was mounted on the suppressor electrode with recessing ceramic rods. Smooth metallic parts in the shape of rings were placed at the bottom of the ceramic rods to decrease the potential gradient and to potentially avoid voltage breakdown at the triple points.

The Faraday cup is made out of graphite, because it has a high melting point. It is tilted in order to spread the heat deposited by the ion beam over a large area. The Faraday cup is equipped with two magnets of opposite polarites which creates a transversal magnetic field to suppress secondary emission electrons caused by the energetic ions hitting the graphite. The strength of the magnetic field between the two magnets is approximately 120 gauss. Most secondary emission electrons have an energy of less than $100 \mathrm{eV}$. For a magnetic field strength of 120 gauss, the cyclotron radius for electrons of $100 \mathrm{eV}$ is $2.8 \mathrm{~mm}$. Given the 25-mm aperture of the Faraday cup, most secondary emission electrons will not escape the cup.

Figure 7.35 is a photograph of the prototype $200 \mathrm{kV}$ accelerator column with the Faraday cup used for the experiments.

\section{Experimental setup for testing the accelerator column}

Figure 7.36 shows a schematic diagram of the experimental setup used to test the high-voltage accelerator column. The extraction electrode is connected to a negative $100-\mathrm{kV}$ power supply via a $100-\mathrm{kV}$ vacuum feed-through. A single negative $250-\mathrm{kV}$ power supply is used for both the suppressor electrode and the Faraday cup. The $250-\mathrm{kV}$ power supply is connected to a voltage divider stack of total resistance $2010 \mathrm{M} \Omega$. The stack is divided into two stages, the resistance of the lower stage is $2000 \mathrm{M} \Omega$, while the one of the upper stage is $10 \mathrm{M} \Omega$. At a voltage of $-210 \mathrm{kV}$, a current of $\sim 100 \mu \mathrm{A}$ is circulating through the 


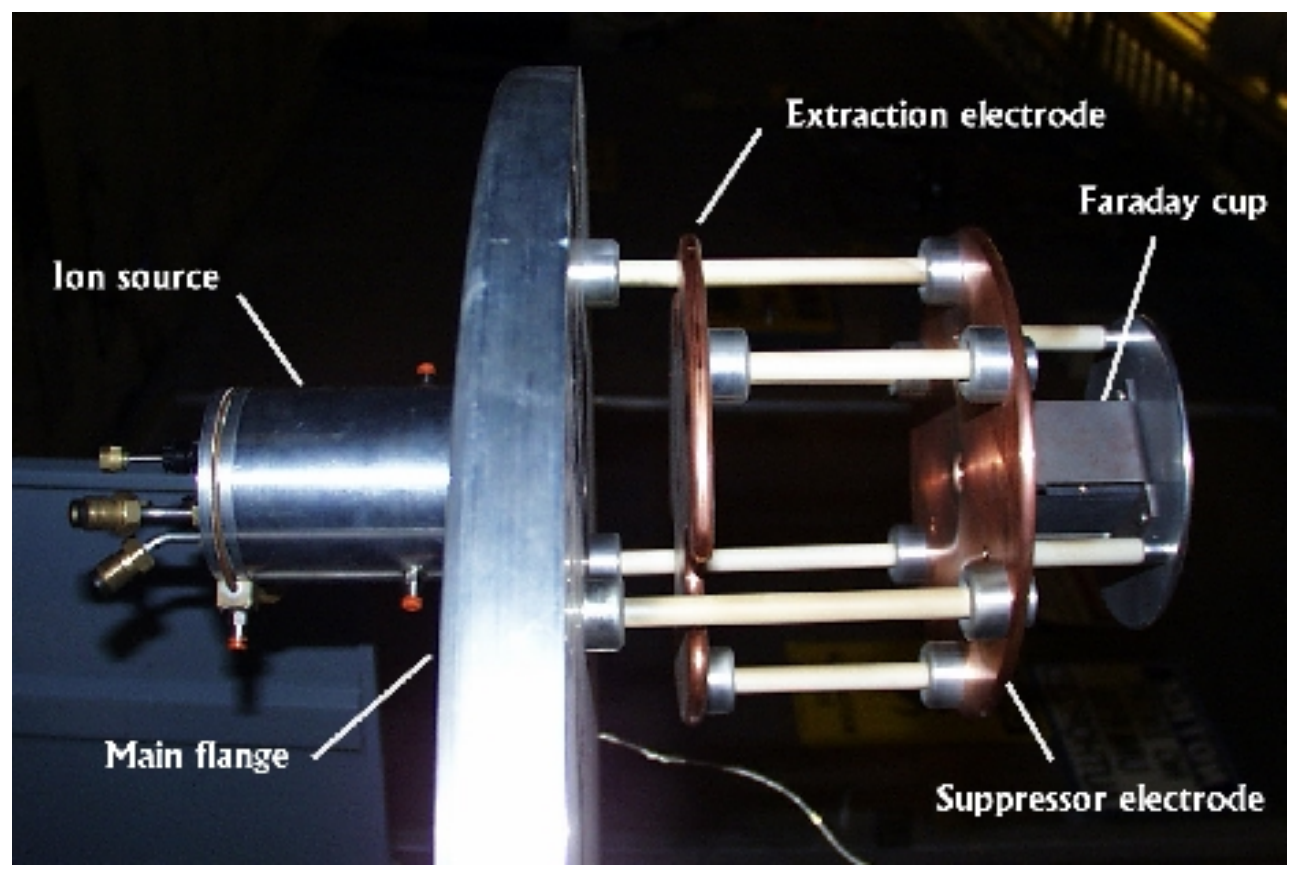

Figure 7.35: Photograph of the accelerator column.

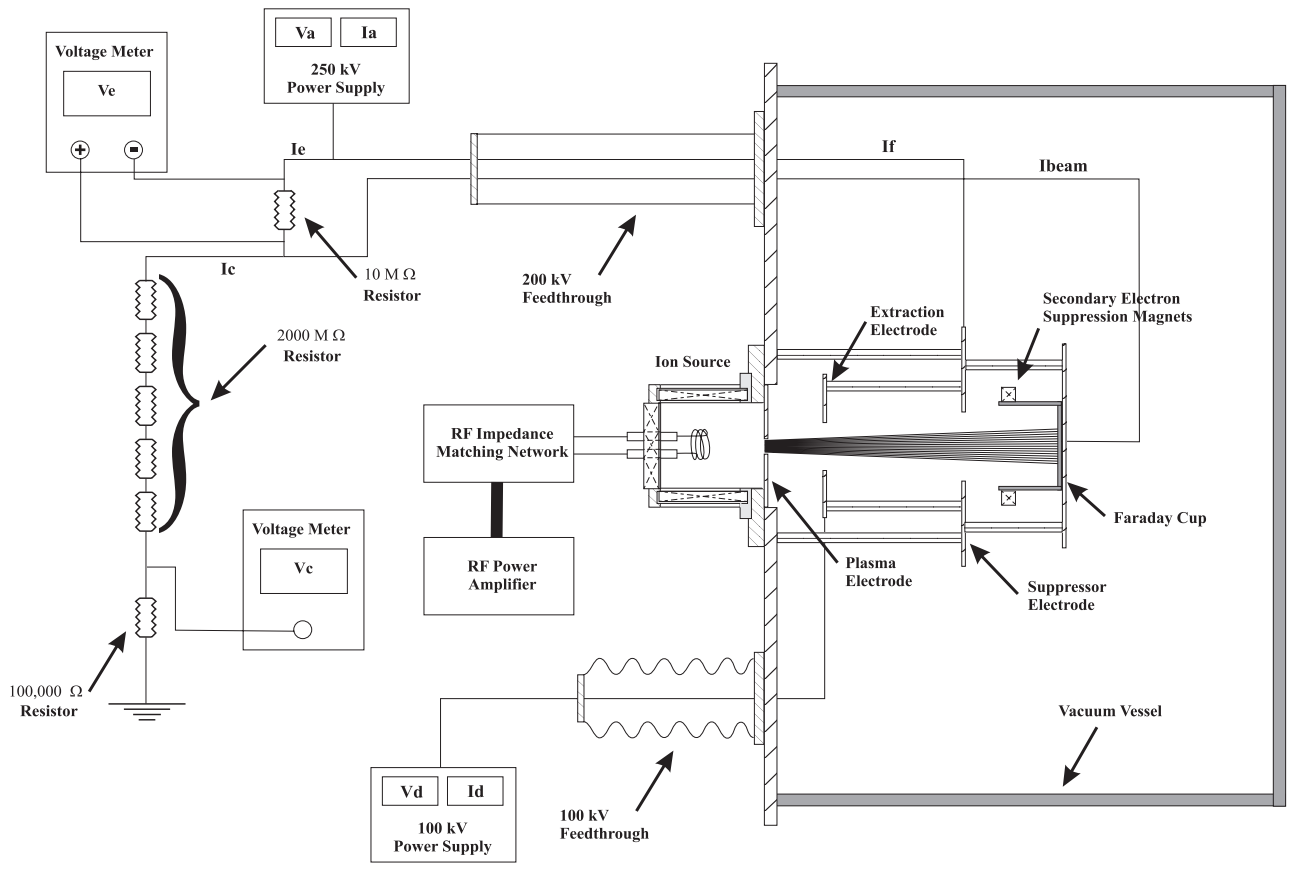

Figure 7.36: Schematic diagram of the experimental setup used for testing the high-voltage accelerator column. 
stack, creating a voltage drop of $1 \mathrm{kV}$ across the $10 \mathrm{M} \Omega$ resistor of the divider stack. The suppressor electrode is connected directly to the $250-\mathrm{kV}$ power supply, while the Faraday cup is connected below the $10 \mathrm{M} \Omega$ resistor of the divider stack. Assuming a 1-m A current in the Faraday cup, the voltage drop in the 10 - $\mathrm{M} \Omega$ resistor in the upper stage of the voltage divider stack is $11 \mathrm{kV}$, making the target float at a potential $11 \mathrm{kV}$ more positive than the Faraday cup. This potential difference provides electrical suppression of secondary emission electrons emitted by the Faraday cup, in addition to the existing magnetic suppression. Because of the small potential difference between the two conductors, a single vacuum feedthrough is used to carry the voltages from the divider stack to the accelerator column. Figure 7.37 shows a photograph of the two high-voltage feed-throughs and the ion source mounted on the main flange, which is bolted onto the vacuum chamber. Figure 7.38 shows a photograph of the ion source on the main flange.

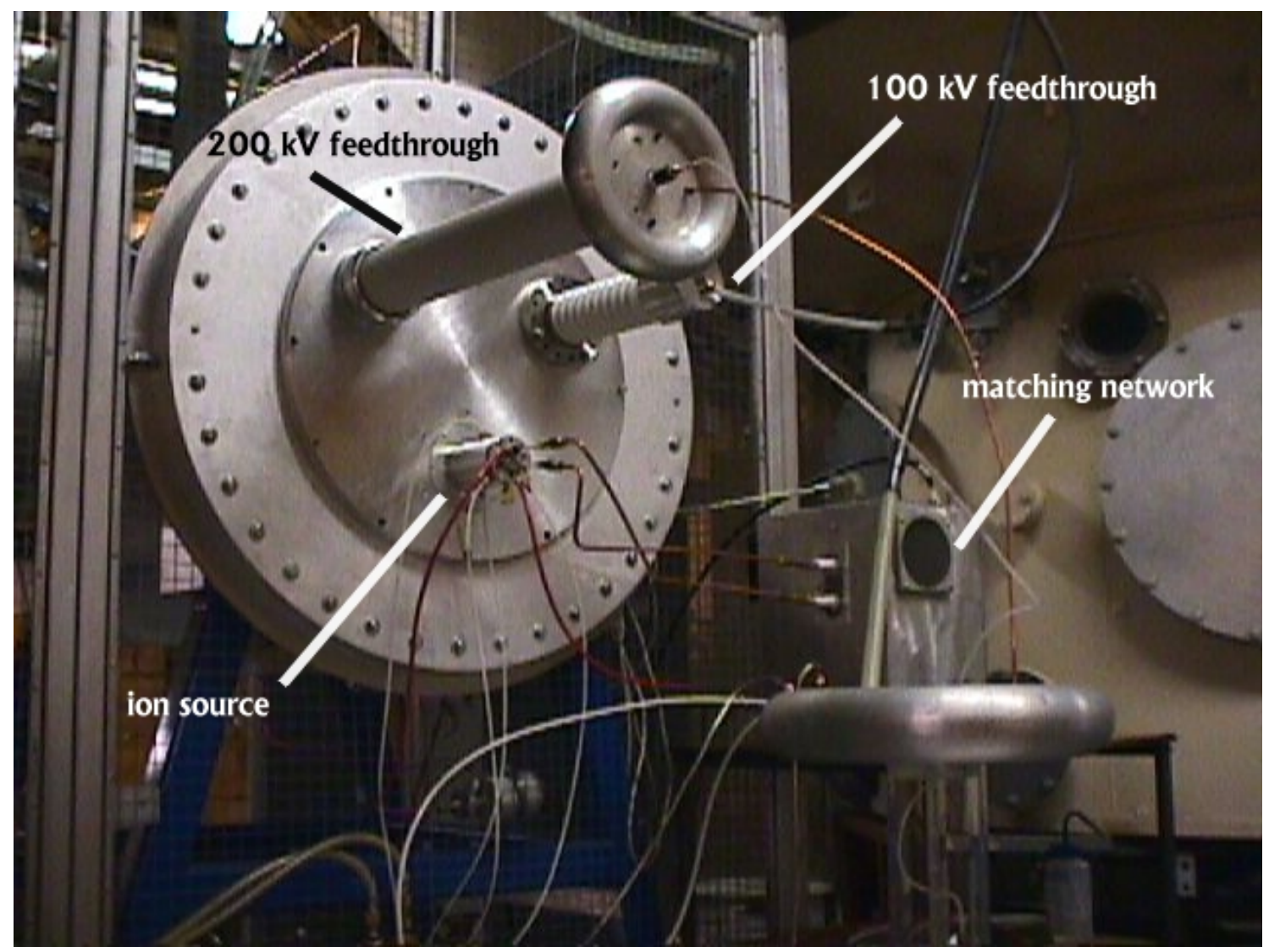

Figure 7.37: View of the experimental setup in the test stand. 


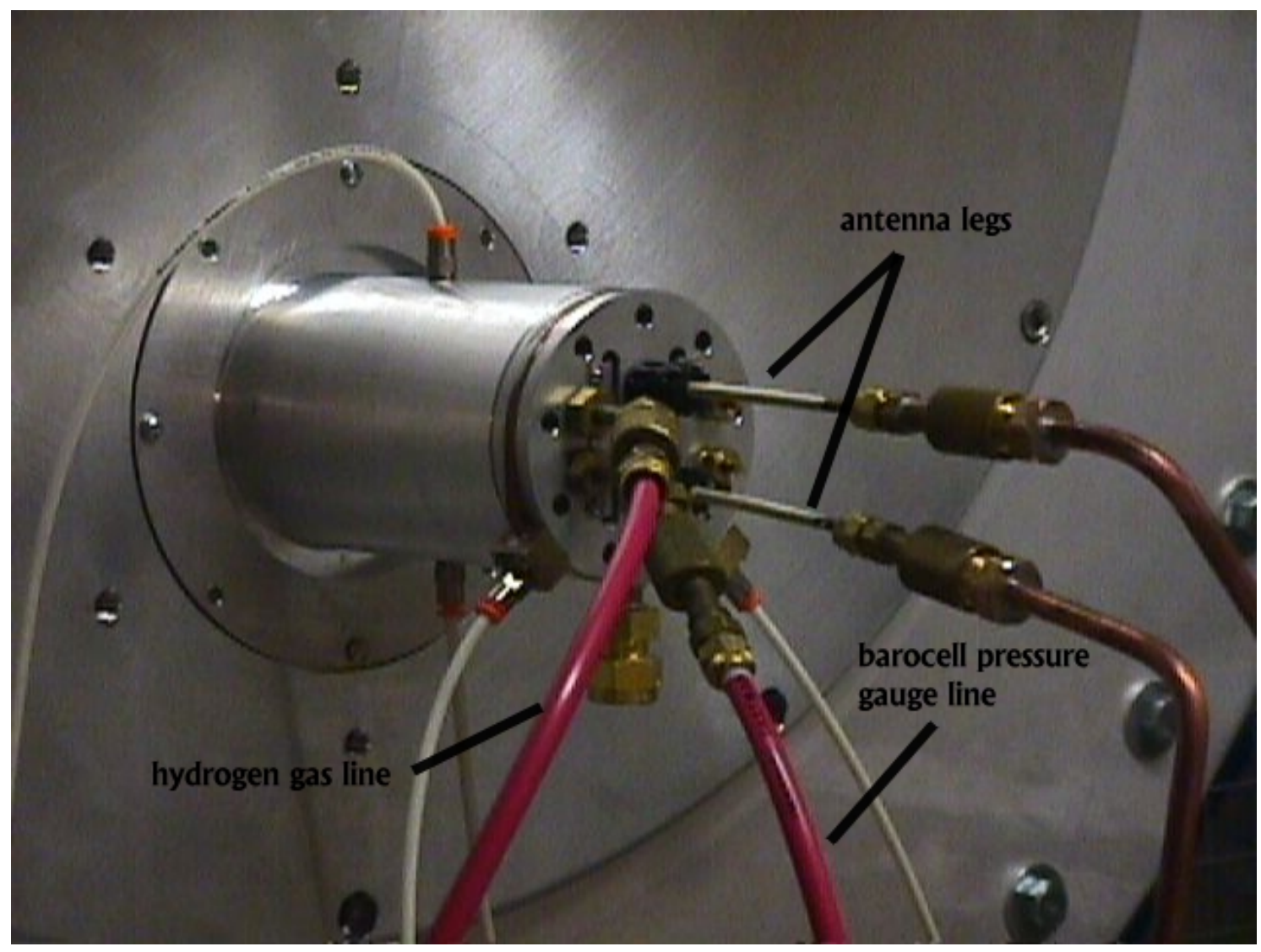

Figure 7.38: View of the ion source in the test stand.

To determine the beam current to the Faraday cup $I_{\text {beam }}$, the voltage drop $V_{e}$ across the $10-\mathrm{M} \Omega$ resistor in the divider stack is recorded, as well as the voltage drop $V_{c}$ across a $100-\mathrm{k} \Omega$ resistor at the bottom of the divider stack. Using the following relationships

$$
\begin{aligned}
I_{c} & =-\frac{V_{c}}{100 \cdot 10^{3}}, \\
I_{e} & =\frac{V_{e}}{10 \cdot 10^{6}}, \\
I_{\text {beam }} & =I_{e}-I_{c},
\end{aligned}
$$

the beam current to the Faraday cup can be calculated. The beam current to the suppressor electrode is calculated using

$$
I_{f}=I_{a}-I_{e}
$$

where $I_{a}$ is the current read on the display of the $250-\mathrm{kV}$ power supply. The beam current to the extraction electrode $I_{d}$ is read directly on the display of the $100-\mathrm{kV}$ power supply. 


\subsubsection{Experimental results}

The prototype accelerator column was first conditioned without ion beam. Conditioning was then performed with a proton beam extracted from the ion source. The 5-cm-diam ion source characterized in Secs. 7.3.1 through 7.3.4 was operated at a pressure of 6 mTorr with a cw RF power of $1900 \mathrm{~W}$ without the magnetic filter. According to the characterization of the ion source in Sec. 7.3.4, these operating conditions lead to a current

density of $27 \mathrm{~mA} / \mathrm{cm}^{2}$ (see Fig. 7.28). With the 2-mm-diam plasma electrode aperture, the extracted current can be calculated and is equal to $0.85 \mathrm{~mA}$. After a considerable amount of time spent on conditioning the accelerator column with the proton beam, the accelerator could be operated up to $165 \mathrm{kV}$. Operation at higher voltages was very difficult due to voltage breakdown. Every spark between components in the vacuum chamber would shut down the non-regulated $250-\mathrm{kV}$ power supply.

Current measurements were performed after conditioning. At very low extraction voltages, large beam currents to the extraction electrode were measured. This corresponds to the case of an over-dense plasma as shown in Fig. 7.31c. The beam current to the extraction electrode decreased as the potential difference between plasma and extraction electrodes was raised. Eventually this current becomes negligible (around $25 \mu \mathrm{A}$ ) compared to the Faraday cup current for a potential difference of 25 to $26 \mathrm{kV}$. This potential difference corresponds to the one predicted by the ion beam optics simulations shown in Fig. 7.32, confirming the validity of the simulations. The plasma meniscus in this case is shown in Fig. $7.31 \mathrm{~b}$.

For larger potential differences, one reaches the under-dense plasma regime (see Fig. 7.31a). In this regime, non-zero currents were measured on both the extraction and the suppressor electrodes. A possible explanation for these currents could be a crossover of the ion beam somewhere between the plasma and the suppressor electrodes. A cross-over results in a fast ion beam expansion beyond the cross-over point, which would itself cause the ion beam to impinge on the suppressor electrode. A fraction of the current measured on the suppressor electrode is the ion beam current. The other fraction is the secondary emission electron current produced by the ion beam bombardment. The secondary emission electrons are accelerated backward and intercepted by the extraction electrode, which explains the current measured on the extraction electrode. As soon as this electron current was measured on the extraction electrode, its potential would rapidly drop from $\sim-25 \mathrm{kV}$ to $-40 \mathrm{kV}$ or even 
lower values until the electron current was becoming too high. At that point, the $250-\mathrm{kV}$ non-regulated power supply would automatically shut down. This rapid potential drop can also be explained. Once secondary electrons are intercepted by the extraction electrode, its potential decreases and leads to an even more under-dense plasma. The cross-over position moves backward, resulting in a larger fraction of the ion beam intercepted by the suppressor electrode, more secondary emission electrons produced and intercepted by the extraction electrode, lower extraction electrode potential, etc.

At the maximum voltage that the accelerator column could hold and with the ion source operating at the conditions specifed above, a $0.76 \mathrm{~mA}$ proton beam was measured in the Faraday cup. This current is very close to the predicted current of $0.85 \mathrm{~mA}$. The extraction electrode current was equal to $50 \mu \mathrm{A}$.

Due to sputtering of the copper electrodes by the energetic ions, the ceramic insulators were soon covered by a conductive layer of copper. Voltage breakdown between neighboring electrodes then became a problem. The problem could be solved by shielding

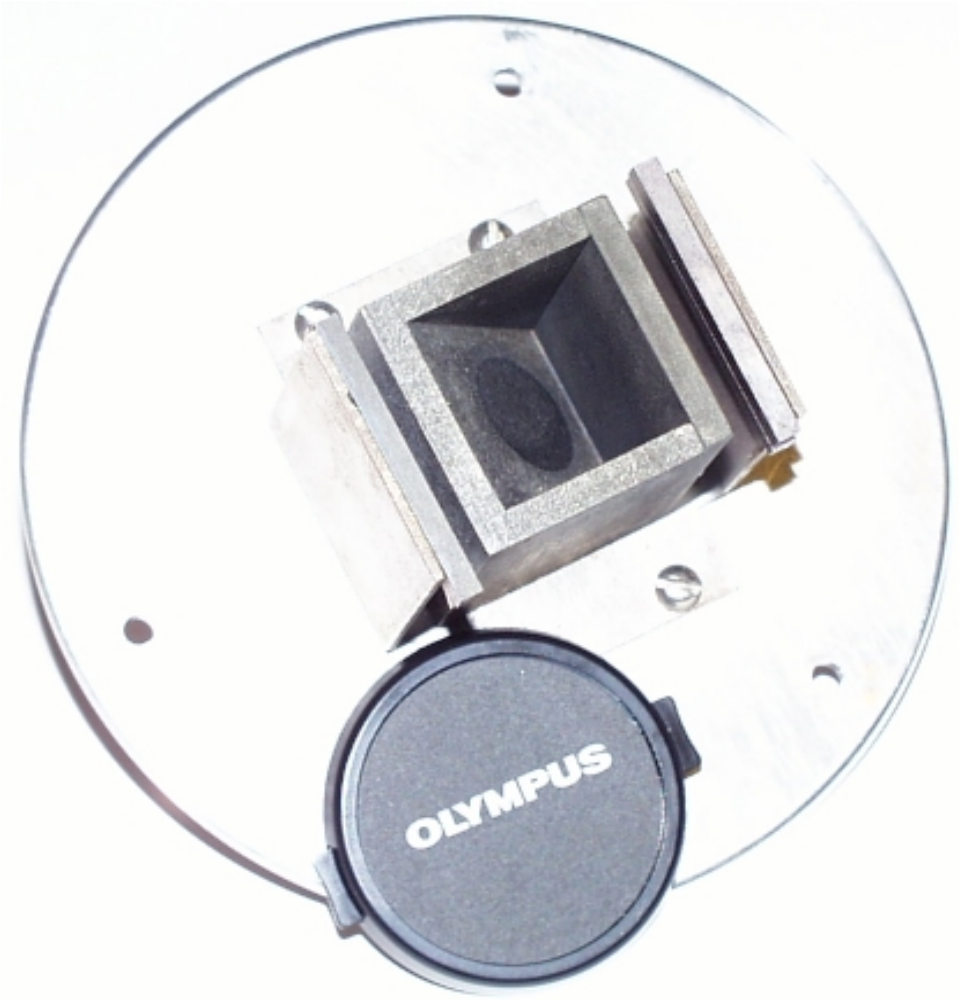

Figure 7.39: Photograph of the Faraday cup showing the beam spot and the two magnets for the secondary emission electron suppression. 
the ceramic rods but these changes were not made, because enough data had been taken. Figure 7.39 shows a photograph of the Faraday cup after the experiment. One can clearly see the beam spot on the graphite beam stop, and the two magnets at the entrance of the Faraday cup aperture.

\subsubsection{Design of higher-current accelerator columns}

For the $15 \mathrm{~mA}, 150 \mathrm{~mA}$ and $1.5 \mathrm{~A}$ beams, the beam extraction system which closes off the other end of the ion source chamber, will consist of a multi-aperture extraction system as represented in Fig. 7.1. In order to get a good beam uniformity on the target, a multi-slit extraction system has been chosen over a multi-hole one. Figure 7.40 shows one of the 6 beamlets in the accelerator column to be used for the $15 \mathrm{~mA}$ and $150 \mathrm{~mA}$ steps.

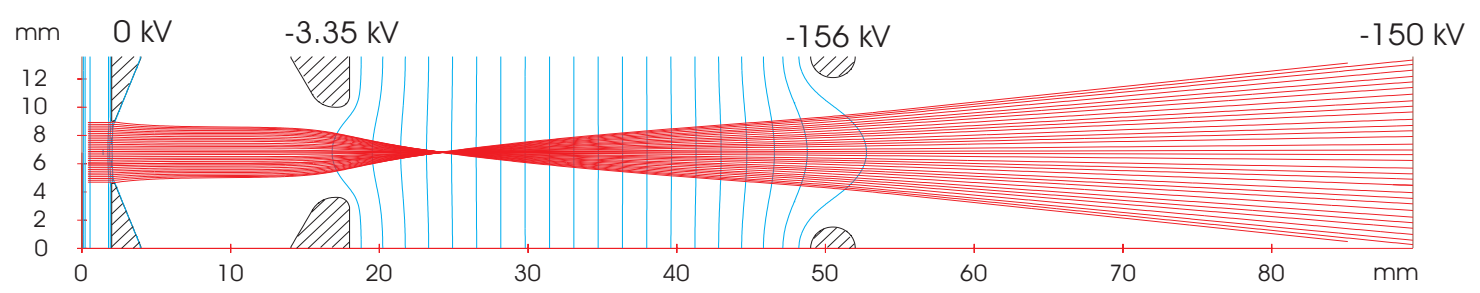

Figure 7.40: Slit type accelerator column design using IGUN simulation code for proton beam. The current density at the ion source is $10 \mathrm{~mA} / \mathrm{cm}^{2}$, the monatomic and diatomic ion species are assumed to be $85 \%$ and $15 \%$.

The 6 beamlets will sum up to a total current of $150 \mathrm{~mA}$. A mask will block 4 slits of the plasma electrode and cover the 2 remaining slits on $2 / 3$ of their length. Experiments will be performed with $15 \mathrm{~mA}$ of beam current. The crossover is needed for the fast expansion of the beamlets after they exit from the suppressor electrode. The expansion of $300 \%$ is essential for the uniform heat load across the target. With the optics shown, the power on the target is approximately $350 \mathrm{~W} / \mathrm{cm}^{2}$ with a uniformity of $\sim 10 \%$. Ion optics simulations were also performed for beams without crossover but they led to accelerator columns five times longer for the same final expansion and current density on the target. However, the beam loss due to charge exchange in this accelerator column was unacceptable.

The ion source, accelerator column and target will be operated with natural hydrogen in the first phase. Ion production in the ion source, ion beam losses in the accelerator column, target loading and cooling will be studied with natural hydrogen. Ion beam loss is a concern in the neutron tube because the accelerator column must operate at the same 
pressure as the ion source in a sealed system. For the accelerator column shown in Fig. 7.40, beam losses due to electron capture reactions $\left(H^{+}+H_{2} \rightarrow H+H_{2}^{+}\right)$have been computed and are shown in Fig. 7.41 for different gas pressures in the accelerator column. Since the

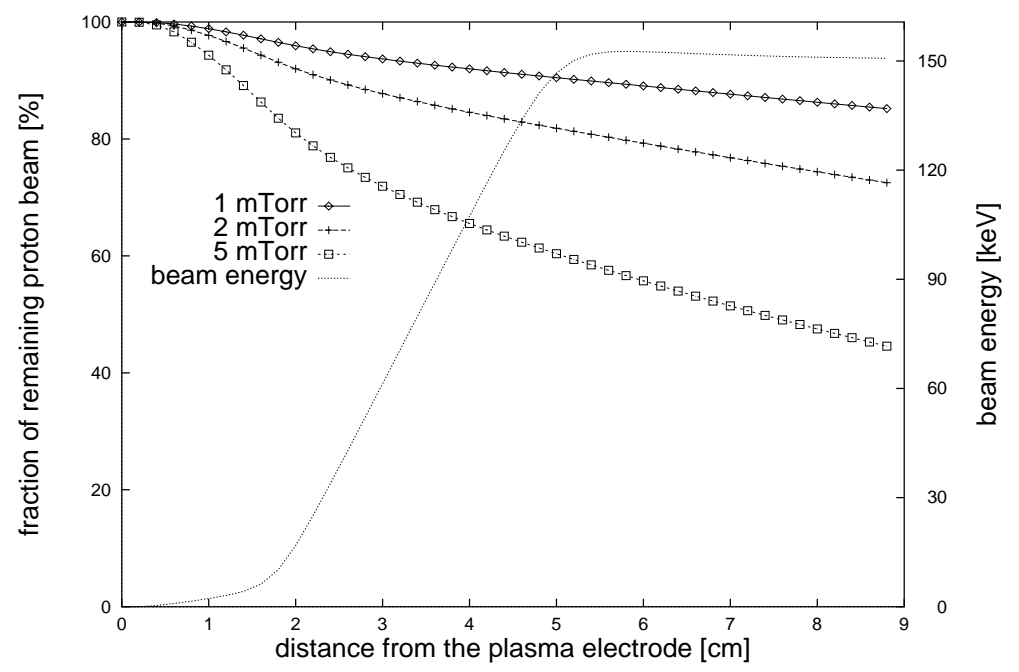

Figure 7.41: Proton beam losses as a function of distance from the plasma electrode and neutral gas pressure.

electron capture reactions do not scatter significantly the incoming $H^{+}$ions, the neutral atoms $H$ formed continue in the same direction as the $H^{+}$ions. Therefore, the $H$ atoms produced by electron capture reactions occuring at energies greater than $150 \mathrm{keV}$ past the suppressor electrode will still reach the target with full energy, and will not contribute to the beam losses. The total beam loss is thus the difference between the beam current at the plasma electrode and the remaining beam current at $5 \mathrm{~cm}$. It is equal to $9 \%, 18 \%$ and $39 \%$ for 1 mTorr, 2 mTorr and 5 mTorr respectively.

One has shown by IGUN simulations that the same accelerator column could be used for deuteron and mixed deuteron/triton beams by just varying the extraction electrode potential within a few kilovolts. The beam losses for these beams were comparable to the ones for proton beams.

\subsubsection{Summary of results}

A prototype 150 to $200-\mathrm{kV}$ accelerator column was designed using the IGUN ion beam transport simulation code to extract and accelerate a 1-mA proton beam onto a target. The accelerator was successfully tested up to $165 \mathrm{kV}$. Higher voltages could not be held due 
to voltage breakdown in the column. At the maximum voltage that the accelerator column could hold, a 0.8 -m A proton beam could be extracted and accelerated onto a graphite target. This beam current was very close to the one predicted by ion beam transport simulation.

Beam loss is a concern in the final sealed neutron generator, because the accelerator column will be operating at the same gas pressure as the ion source. Using a 8-cm-long accelerator column, beam losses of $9 \%, 18 \%$ and $39 \%$ were computed for gas pressures of 1, 2 and 5 mTorr respectively. In order to minimize beam losses and to provide enough ion current, one has to compromise the source operation with a pressure $\leq 2 \mathrm{~m}$ Torr. 


\section{Chapter 8}

\section{Conclusion}

The most important results of this thesis are summarized in this Chapter. It is divided into three parts. The first one relates to boron neutron capture therapy. It summarizes the guidelines obtained by numerical simulations for the treatment of shallow and deep-seated brain tumors, as well as the results on the design of beam-shaping assemblies to moderate D-D and D-T neutrons to epithermal energies. The second part is about boron neutron capture synovectomy for the treatment of rheumatoid arthritis. Optimal neutron energy for treatment and beam-shaping assembly designs are summarized in this section. The last part is on the development of the sealed neutron generator, including experimental results on the prototype ion source and the prototype accelerator column.

\subsection{Boron neutron capture therapy}

The first part is on boron neutron capture therapy (BNCT) for the treatment of brain tumors. A monoenergetic neutron beam simulation study was carried out to determine the most suitable neutron energy for treatment of shallow and deep-seated brain tumors. Two figures-of-merit - the absorbed skin dose and the absorbed tumor dose at a given depth in the brain — were used to measure the neutron beam quality. For deep-seated tumors, the energy range 1 to $20 \mathrm{keV}$ appeared to be optimal, with a maximum therapeutic gain at $\sim 8 \mathrm{keV}$. For shallow tumors, any neutron beam of energy higher than 1 to $10 \mathrm{eV}$ and lower than $\sim 8 \mathrm{keV}$ resulted in a relatively high therapeutic gain. The effect of the neutron beam diameter on the tumor dose was very small for shallow tumors, but it was considerably large for deep-seated tumors. For instance, the tumor dose at a depth of $8 \mathrm{~cm}$ more than 
doubled when the neutron beam diameter increased from 6 to $18 \mathrm{~cm}$. The drawback of larger diameter beams is that the volume of high healthy-tissue dose also increases with the beam diameter. Therefore, we conclude that no set of neutron beam diameter and energy is best in all respects for any kind of tumors. Diameter and energy of the neutron beam have to be determined by the characteristics of the tumor to be treated, such as tumor depth, size, and spread in the surrounding tissues.

Based on the results of this neutron beam study, moderators, reflectors and delimiters were designed and optimized to moderate the high-energy neutrons from the fusion reactions D-D and D-T down to a suitable energy spectrum. Two different computational models (MCNP and BNCT_RTPE) were used to study the dose distribution in the brain. The first method was based on the simulation of the radiation transport in the beam-shaping assembly (BSA) and the phantom with MCNP. The second method was based on the coupling of MCNP and BNCT_RTPE for the radiation transport simulations in the BSA and phantom, respectively, the coupling being done through an exit window between the BSA and the phantom. The first method was more accurate but also more time consuming.

Our analysis showed that the low neutron yield of the D-D reaction was not favorable for the treatment of glioblastoma multiforme. On the other hand, high-energy neutrons from the D-T reaction could be moderated to the desired energy range without reducing the neutron flux to a negligible level. With the optimal beam-shaping assembly, a 1.5-A mixed deuteron/triton beam of energy $150 \mathrm{keV}$ accelerated onto a titanium target led to a treatment time of $\sim 45 \mathrm{~min}$. The dose near the center of the brain obtained with this configuration was more than $65 \%$ higher than the dose from a typical spectrum produced by the Brookhaven Medical Research Reactor, and was comparable to the dose obtained by other accelerator-produced neutron beams.

\subsection{Boron neutron capture synovectomy}

In the second part of this thesis, boron neutron capture synovectomy (BNCS) was investigated for the treatment of rheumatoid arthritis. In order to determine the optimal neutron energy for treatment, a monoenergetic neutron beam simulation study was carried out using two figures-of-merit to measure the neutron beam quality, the ratio of the synovium absorbed dose to the skin absorbed dose, and the ratio of the synovium absorbed dose to the bone absorbed dose. Thermal neutron beams were found to be optimal. 
Similar absorbed dose rates and therapeutic ratios were obtained with monodirectional and isotropic neutron beams. The thermal neutron flux required to deliver 100 Gy-equivalent to the synovium in $10 \mathrm{~min}$ is approximately $2.8 \times 10^{8} \mathrm{n} / \mathrm{cm}^{2} / \mathrm{s}$.

Computation of the dose distribution in the knee requires the simulation of the neutron and photon transport from the neutron source to the knee phantom through the complex beam-shaping assembly. A method was developed to predict the absorbed dose distribution from any neutron and photon beam spectra incident on the knee. This method is reasonably accurate and it enables one to reduce by a factor 10 the neutron and photon transport simulation time by modeling the transport in the beam-shaping assembly only. Good agreement was observed between dose distributions computed by this method and those computed by using simulation of the entire model, i.e., beam-shaping assembly and knee phantom.

Beam-shaping assemblies were designed to moderate the neutrons of the D-D and

$\mathrm{D}-\mathrm{T}$ fusion reactions using the method mentioned above. Good therapeutic ratios were obtained with the D-D reaction but the treatment time was longer than $2 \mathrm{~h}$ for a neutron yield at the target of $7 \times 10^{11} \mathrm{n} / \mathrm{s}$. It can potentially be reduced if the ${ }^{10} \mathrm{~B}$ concentration in the synovium is increased. For D-T neutrons, high therapeutic ratios and treatment times of approximately $5 \mathrm{~min}$ were obtained for neutron yields at the target of $10^{14} \mathrm{n} / \mathrm{s}$. This short treatment time makes the D-T reaction attractive for BNCS. An increase in the beamshaping assembly diameter had a beneficial effect on the treatment time and therapeutic ratios.

\subsection{Neutron generator}

In the third part, the neutron generator along with its main components, i.e., the ion source, the accelerator and the target, were described. The neutron generator based on the $\mathrm{D}$ - $\mathrm{T}$ reaction is sealed in a metal container without external pumping to avoid release of radioactive tritium.

Thick-target neutron yield computations were performed to estimate the neutron yield of titanium and scandium targets. For D-D, neutron yields of about $7 \times 10^{11} \mathrm{n} / \mathrm{s}$ were obtained with a 1.5- A, 150-keV mainly monatomic deuteron beam bombarding deuterated targets. For D-T, with an average deuteron beam current of $1 \mathrm{~A}$ and an energy of 120 $\mathrm{keV}$, a neutron production of about $10^{14} \mathrm{n} / \mathrm{s}$ was estimated for a tritiated target. In mixed 
deuteron/triton beam operation, a beam current of $2 \mathrm{~A}$ at $150 \mathrm{keV}$ was required for the same neutron output. Despite this lower neutron production, this mode of operation is however advantageous because the target lifetime is increased from a few hours to more than $1000 \mathrm{~h}$. Cooling of the target was found to be essential for the neutron yield. Because of the greater thermal stability of scandium hydride, scandium targets lead to greater neutron yields than titanium targets, especially at higher temperatures. The neutron yield in general decreased rapidly with the fraction of molecular (as opposed to monatomic) ion species bombarding the target.

With hydrogen ions, monatomic fractions higher than $95 \%$ were achieved using a prototype 5 -cm-diam RF-driven multicusp source. The ion source could be operated at low gas pressures ( 1 to 2 mTorr). Low gas pressures are necessary to reduce both charge exchange and high-voltage breakdown in the accelerator column. The beam loss is of great concern because the accelerator column will be operating at the same gas pressure - a few mTorrs - as the ion source in the sealed neutron generator. Using a 8-cm-long accelerator column, beam losses of $9 \%, 18 \%$ and $39 \%$ were computed for gas pressures of 1,2 and 5 mTorr respectively.

A prototype 150 to $200-\mathrm{kV}$ accelerator column was successfully tested up to 165 $\mathrm{kV}$. A 0.8 -m A proton beam was extracted and accelerated onto a graphite target with this energy.

These experimental findings will enable us to develop high-intensity, sealed compact neutron generators. 


\section{Bibliography}

[1] J. Chadwick, Nature, 129, 312 (1932).

[2] R.F. Barth, A.H. Soloway, R.G. Fairchild, and R.M. Brugger, "Boron neutron capture therapy: realities and prospects," Cancer, 70, 2995-3001 (1992).

[3] G.L. Locher, "Biological effects and therapentic possibilities of neutrons," Am. J. Roentgenol., 36, 1-13 (1936).

[4] H.J. Taylor, and M. Goldhaber, "Detection of nuclear disintegration in a photographic emulsion", Nature, 135, 341 (1935).

[5] E. Binello, S. Shortkroff, A. Jones, C. Viveiros, A. Ly, C.B. Sledge, A. Davidson, R.E. Shefer, and J.C. Yanch, "Research in Boron Neutron Capture Synovectomy," Proc. Int. Conf. Neutrons in Research and Industry, Crete, Greece, June 9-15, 1996, SPIE 2867, p. 68-71 (1996).

[6] J.C. Yanch, X.-L. Zhou, and G.L. Brownell, "A Monte-Carlo Investigation of the Dosimetric Properties of Monoenergetic Neutron Beams for Neutron Capture Therapy," Radiation Res., 126, 1-20 (1991).

[7] J. Csikai, Handbook of Fast Neutron Generators, vol. 1, CRC Press, Inc., Boca Raton, Florida (1987).

[8] J.B. Marion, and J.L. Fowler, Fast Neutron Physics, Part I, Monographs and Texts in Physics and As J.B. Marion and J.L. Fowler, Eds., Interscience Publishers, New York (1960).

[9] Private communication with Nicola Colonna, INFN, Nari, Italy.

[10] D.L. Bleuel, R.J. Donahue, B.A. Ludewigt, and J. Vujic, "Designing Accelerator-Based Epithermal Neutron Beams for BNCT," Med. Phys., 25, 9, 1725-1734 (1998). 
[11] J.C. Yanch, R.E. Shefer, and E. Binello, "Design of LowEnergy Neutron Beams for Boron Neutron Capture Synovectomy," Proc. Int. Conf. Neutrons in Research and Industry, Crete, Greece, June 9-15, 1996, SPIE 2867, p. 31-40 (1996).

[12] High Voltage Engineering Corporation, Burlington, Massachusetts.

[13] "Measurement of Absorbed Dose of Neutrons and Mixtures of Neutrons and Gamma Rays," National Bureau of Standards Handbook 75, Washington, D.C. (1961).

[14] "Photon, Electron, Proton and Neutron Interaction Data for Body Tissues," ICRU 46, International Commission on Radiation Units and Measurements (1992).

[15] R.G. Zamenhof, S.D. Clement, O.K. Harling, J.K. Brenner, D.E. Wazer, H. Madoc-Jones, and J.C. Yanch, "Monte Carlo Based Dosimetry and Treatment Planning for Neutron Capture Therapy of Brain Tumors," Neutron Beam Design, Development, and Performance for Neutron Capture Therapy, O.K. Harling, J.A. Bernard, and R.G. Zamenhof, Eds., in Basic Life Sciences, vol. 54, p. 283, Plenum Press, New York (1990).

[16] R.S. Caswell, J.J. Coyne, and M.L. Randolph, "KERMA Factors of Elements and Compounds for Neutron Energies Below $30 \mathrm{MeV}$, Int. J. Appl. Radiat. Isot., 33, 1227 $1262(1982)$.

[17] A.D. Chanana, "Boron Neutron Capture Therapy of Glioblastoma Multiforme at the Brookhaven Medical Research Reactor, A Phase I/II Study," FDA IND 43317, Protocol 4, Brookhaven National Laboratory (1996).

[18] Y. Mishima, M. Ichihashi, S. Hatta, C. Honda, K. Yamamura, and T. Nakagawa, "New Thermal Neutron Capture Therapy for Malignant Melanoma: MelanogenesisSeeking ${ }^{10}$ B Molecule-Melanoma Cell Interaction from In Vitro to First Clinical Trial," Pigment Cell Res., 2, 226-234 (1989).

[19] A.H.W. Nias, An Introduction to Radiobiology, John Wiley \& Sons, New York (1990).

[20] J.F. Briesmeister, "MCNP - A General Monte Carlo N-Particle Transport Code, Version 4B," LA-12625-M, Los Alamos National Laboratory (1997). 
[21] W.S. Snyder, M.R. Ford, and G.G. Ford, "Estimates of Specific Absorbed Fractions for Photon Sources Uniformly Distributed in Various Organs of a Heterogeneous Phantom,” MIRD Pamphlet 5 Revised, Society of Nuclear Medicine, New York (1978).

[22] C.-K.C. Wang, T.E. Blue, and R. Gahbauer, "A Neutronic Study of an Accelerator-Based Neutron Irradiation Facility for Boron Neutron Capture Therapy," Nucl. Technol., 84, 93-107 (1989).

[23] J.C. Yanch, X.L. Zhou, R.E. Shefer, and R.E. Klinkowstein, "Accelerator-Based Epithermal Neutron Beam Design for Neutron Capture Therapy," Med. Phy., 19, 709-721 (1992).

[24] D.A. Allen, and T.B. Beynon, “A Design Study for an Accelerator-Based Epithermal Neutron Beam for BNCT," Phys. Med. Biol., 40, 807-821 (1995).

[25] J.E. Woollard, T.E. Blue, N. Gupta, and R.A. Gahbauer, "Evaluation of Moderator Assemblies for Use in an Accelerator-Based Neutron Source for Boron Neutron Capture Therapy," Nucl. Technol., 123, 320-334 (1998).

[26] D.L. Bleuel, S.V. Costes, R.J. Donahue, B.A. Ludewigt, and J. Vujic, "Neutronics Analysis of Three Beam-Filter Assemblies for an Accelerator-Based BNCT Facility," Proc. Topl. Mtg. Nuclear Applications of Accelerator Technology, Albuquerque, New Mexico, November 16-20, 1997, p. 502-507, American Nuclear Society (1997).

[27] D.L. Bleuel, R.J. Donahue, and B.A. Ludewigt, "On Optimizing the ${ }^{7} \operatorname{Li}(p, n)$ Proton Beam Energy and Moderator Material for BNCT," presented at the 14th Int. Conf. Application of Accelerators in Research and Industry, Denton, Texas, November 6-9, 1996, LBNL-39057 UC-413, Lawrence Berkeley National Laboratory (1996).

[28] D. Nigg, "Methods for Radiation Dose Analysis and Treatment Planning in BNCT," Int. J. Radiat. Oncol. Biol. Phys., 28, 1121-1134 (1994).

[29] H.B. Liu, D.D. Greenberg, J. Copala, and F.J. Wheeler, “An Improved Neutron Collimator for Brain Tumor Irradiations in Clinical Boron Neutron Capture Therapy," Med. Phys., 23, 2051-2060 (1996).

[30] J.A. Coderre, R. Bergland, J. Capala, M. Chadha, A.J. Chanana, E. Elowitz, D.D. Joel, H.B. Liu, and D. Slatkin, "Boron Neutron Capture Therapy for Glioblastoma 
Multiforme Using p-boronophenylalanine and Epithermal Neutrons - Trial Design and Early Clinical Results," J. Neuro-Oncol., 33, 93-104 (1997).

[31] T. Goorley, F. Wheeler, J. Capala, W.S. Kiger III, M. Palmer, and R. Zamenhof, "A Comparison of Two Treatment Planning Programs: MacNCTPlan and BNCT_RTPE," Proc. 8th Int. Symp. Neutron Capture Therapy for Cancer, La Jolla, California, September 13-18, 1998, Plenum Publishing Corporation (1998).

[32] D..J. Wilde, and C.S. Beightler, Foundations of Optimization, Prentice-Hall, Englewood Cliffs, N.J. (1967).

[33] J.J. Duderstadt, and L.J. Hamilton, Nuclear Reactor Analysis, John Wiley \& Sons, New York (1976).

[34] Y. Karni, E. Greenspan, J. Vujic, and B. Ludewigt, "Optimal Beam-Shaping Assemblies for BNCT Facilities," Trans. Am. Nucl. Soc., 73, 29 (1995).

[35] I. Auterinen, and P. Hiismaki, "Design of an Epithermal Neutron Beam for the TRIGA Reactor in Otaniemi," Proc. CLINCT BNCT Workshop, Helsinki, Finland, 1993, TKK-F-A718, p. 14-24 (1994).

[36] J.M. Verbeke, S.V. Costes, D. Bleuel, J. Vujic, and K.N. Leung, "Designing an Epithermal Neutron Beam for Boron Neutron Capture Therapy for the Fusion Reactions ${ }^{2} \mathrm{H}(d, n)^{3} \mathrm{He}$ and ${ }^{3} \mathrm{H}(d, n)^{4} \mathrm{He}, "$ Proc. Int. Conf. Physics of Nuclear Science and Technology, Long Island, New York, October 5-8, 1998 vol.2, p. 1600, American Nuclear Society (1998).

[37] M. Davis, M. Chinol, "Radiopharmaceuticals for Radiation Synovectomy: Evaluation of Two Yttrium-90 Particulate Agents," J. Nucl. Med., 30, 1047-1055 (1989).

[38] J. Noble, A.G. Jones, M.A. Davis, C.B. Sledge, R.I. Kramer, E. Livni, "Leakage of Radioactive Particle Systems from a Synovial Joint Studied with a Gamma Camera," J. Bone Joint Surg. (Am), 65-A, 381-389 (1983).

[39] J.C. Yanch, S. Shortkroff, R.E. Shefer, S. Johnson, E. Binello, D. Gierga, A.G. Jones, G. Young, C. Vivieros, A. Davidson, and C. Sledge, "Boron Neutron Capture Synovectomy: Treatment of Rheumatoid Arthritis Based on the ${ }^{10} \mathrm{~B}(n, \alpha){ }^{7} \mathrm{Li}$ Nuclear Reaction," Med. Phys., 26, 364-375 (1999). 
[40] Harris, D. Edward, Rheumatoid Arthritis, Saunders, Philadelphia (1997).

[41] D.T. Ingersoll, C.O. Slater, E.L. Redmond, II, and R.G. Zamenhof, "Comparison of TORT and MCNP Dose Calculations for BNCT Treatment Planning," Proc. $7^{\text {th }}$ Int. Symp. Neutron Capture Therapy for Cancer, Zurich, Switzerland, Sept. 4-7, 1996, Trans. American Nuclear Society, vol. 75, 36-37 (1996).

[42] J.M. Verbeke, J. Vujic, and K.N. Leung, "Neutron Beam Optimization for BNCT using the D-D and D-T High Energy Neutron Sources," Nucl. Tech., 129 (2), 257-278 (2000).

[43] L.T. Perkins, P.R. Herz, K.N. Leung, and D. Pickard, Rev. Sci. Instrum., 65, 1186 (1996).

[44] L.T. Perkins et al., Rev. Sci. Instrum., 67, 1057 (1996).

[45] L.A. Shope, "Theoretical Thick Target Yields for the D-D, D-T, and T-D Nuclear Reactions Using the Metal Occluders Ti and Er and Energies up to $300 \mathrm{keV}$," SC-TM66-247, Sandia Laboratory (1966).

[46] J. Kim, "Neutron Sources Using D-T Mixed Beams Driven Into Solid Target," Nucl. Instr. and Meth., 145, 9-17 (1977).

[47] H.H. Andersen, C.C. Hanke, H. Simonsen, H. Sorensen, and P. Vajda, "Stopping Power of the Elements $\mathrm{Z}=20$ Through $\mathrm{Z}=30$ for $5-12 \mathrm{MeV}$ Protons and Deuterons," Phys. Rev., 175, 389 (1968).

[48] J.A. Phillips, "The Energy Loss of Low Energy Protons in Some Gases," Phys. Rev., 90, $532(1953)$.

[49] H.K. Reynolds, D.N.F. Dunbar, W.A. Wenzel, and W. Whaling, "The Stopping Cross Section of Gases for Protons, 30-600 keV," Phys. Rev., 92, 742 (1953).

[50] M.L. Lieberman, and P.G. Wahlbeck, "The Thermodynamics of the ScandiumHydrogen System," J. Phys. Chem., 69, 3514-3519 (1965).

[51] L.C. Beavis, "Characteristics of Some Binary Tansition Metal Hydrides," J. Less-Common Metals, 19, 315-328 (1969).

[52] J. Detaint, M. Raffin, et A. Rey, "Cibles Neutrinogenes a Hydrures Metalliques Fonctionnement et Preparation," Le Vide, 154, Juillet-Aout-Septembre-Octobre (1971). 
[53] K.W. Ehlers, and K.N. Leung, Rev. Sci. Instrum., 52 (10), 1452 (1983).

[54] C.D. Child, Phys. Rev. (Ser. 1), 32, 492 (1911).

[55] R. Becker, "New features in the Simulation of Ion Extraction with Igun," Proc. EPAC 98, Stockolm, Sweden (1998). 


\title{
Appendix A
}

\section{Glossary}

\author{
AR : Atomic Ratio \\ BMRR : Brookhaven Medical Research Reactor \\ BNCS : Boron Neutron Capture Synovectomy \\ BNCT : Boron Neutron Capture Therapy \\ BNCT-RTPE: Boron Neutron Capture Therapy Radiation Treatment Planning Environment \\ BNL : Brookhaven National Laboratory \\ BSA : Beam-Shaping Assembly \\ CF : Compound Factor \\ D-D : Deuterium-Deuterium fusion reaction \\ D-T : Deuterium-Tritium fusion reaction \\ ICRU : International Commission on Radiation Units and Measurments \\ KERMA : Kinetic Energy Released per unit MAss \\ LBNL : Lawrence Berkeley National Laboratory \\ MCNP : Monte Carlo N-Particle transport code \\ MIRD : Medical Internal Radiation Dose Committee of the Society of Nuclear Medicine \\ PTC : Pressure-Temperature-Composition \\ RBE : Relative Biological Effectiveness \\ RF : Radio Frequency
}




\section{Appendix B}

\section{Fluence-to-KERMA conversion}

\section{factors for neutrons, photon mass}

\section{attenuation coefficients}

B.1 Fluence-to-KERMA conversion factors for neutrons in tissues, taken from the International Commission on Radiation Units and Measurements (ICRU) Report No. 46 [14].

\begin{tabular}{|c|c|}
\hline Neutron energy & fluence-to-KERMA conversion factor \\
\hline$[$ MeV] & {$\left[\right.$ rad $\left.\cdot \mathrm{cm}^{2} / \mathrm{n}\right]$} \\
\hline \hline $2.53 \mathrm{e}-08$ & $2.08 \mathrm{e}-11$ \\
\hline $3.60 \mathrm{e}-08$ & $1.76 \mathrm{e}-11$ \\
\hline $6.30 \mathrm{e}-08$ & $1.33 \mathrm{e}-11$ \\
\hline $1.10 \mathrm{e}-07$ & $1.01 \mathrm{e}-11$ \\
\hline $2.00 \mathrm{e}-07$ & $7.49 \mathrm{e}-12$ \\
\hline $3.60 \mathrm{e}-07$ & $5.58 \mathrm{e}-12$ \\
\hline $6.30 \mathrm{e}-07$ & $4.23 \mathrm{e}-12$ \\
\hline $1.10 \mathrm{e}-06$ & $3.20 \mathrm{e}-12$ \\
\hline $2.00 \mathrm{e}-06$ & $2.39 \mathrm{e}-12$ \\
\hline $3.60 \mathrm{e}-06$ & $1.80 \mathrm{e}-12$ \\
\hline $6.30 \mathrm{e}-06$ & $1.40 \mathrm{e}-12$ \\
\hline $1.10 \mathrm{e}-05$ & $1.12 \mathrm{e}-12$ \\
\hline $2.00 \mathrm{e}-05$ & $9.54 \mathrm{e}-13$ \\
\hline
\end{tabular}




\begin{tabular}{|c|c|}
\hline Neutron energy & fluence-to-KERMA conversion factor \\
\hline$[\mathrm{MeV}]$ & {$\left[\mathrm{rad} \cdot \mathrm{cm}^{2} / \mathrm{n}\right]$} \\
\hline $3.60 \mathrm{e}-05$ & $9.25 \mathrm{e}-13$ \\
\hline $6.30 \mathrm{e}-05$ & $1.06 \mathrm{e}-12$ \\
\hline $1.10 \mathrm{e}-04$ & $1.43 \mathrm{e}-12$ \\
\hline $2.00 \mathrm{e}-04$ & $2.27 \mathrm{e}-12$ \\
\hline $3.60 \mathrm{e}-04$ & $3.83 \mathrm{e}-12$ \\
\hline $6.30 \mathrm{e}-04$ & $6.50 \mathrm{e}-12$ \\
\hline $1.10 \mathrm{e}-03$ & $1.12 \mathrm{e}-11$ \\
\hline $2.00 \mathrm{e}-03$ & $2.01 \mathrm{e}-11$ \\
\hline $3.60 \mathrm{e}-03$ & $3.59 \mathrm{e}-11$ \\
\hline $6.30 \mathrm{e}-03$ & $6.16 \mathrm{e}-11$ \\
\hline $1.10 \mathrm{e}-02$ & $1.04 \mathrm{e}-10$ \\
\hline $2.00 \mathrm{e}-02$ & $1.81 \mathrm{e}-10$ \\
\hline $3.60 \mathrm{e}-02$ & $2.99 \mathrm{e}-10$ \\
\hline $6.30 \mathrm{e}-02$ & $4.65 \mathrm{e}-10$ \\
\hline $8.20 \mathrm{e}-02$ & $5.62 \mathrm{e}-10$ \\
\hline $8.60 \mathrm{e}-02$ & $5.81 \mathrm{e}-10$ \\
\hline $9.00 \mathrm{e}-02$ & $5.99 \mathrm{e}-10$ \\
\hline $9.40 \mathrm{e}-02$ & $6.17 \mathrm{e}-10$ \\
\hline $9.80 \mathrm{e}-02$ & $6.35 \mathrm{e}-10$ \\
\hline $1.05 \mathrm{e}-01$ & $6.65 \mathrm{e}-10$ \\
\hline $1.15 \mathrm{e}-01$ & $7.06 \mathrm{e}-10$ \\
\hline $1.25 \mathrm{e}-01$ & $7.45 \mathrm{e}-10$ \\
\hline $1.35 \mathrm{e}-01$ & $7.82 \mathrm{e}-10$ \\
\hline $1.45 \mathrm{e}-01$ & $8.18 \mathrm{e}-10$ \\
\hline $1.55 \mathrm{e}-01$ & $8.51 \mathrm{e}-10$ \\
\hline $1.65 \mathrm{e}-01$ & $8.83 \mathrm{e}-10$ \\
\hline $1.75 \mathrm{e}-01$ & $9.15 \mathrm{e}-10$ \\
\hline $1.85 \mathrm{e}-01$ & $9.44 \mathrm{e}-10$ \\
\hline $1.95 \mathrm{e}-01$ & $9.73 \mathrm{e}-10$ \\
\hline $2.10 \mathrm{e}-01$ & $1.02 \mathrm{e}-09$ \\
\hline $2.30 \mathrm{e}-01$ & $1.07 \mathrm{e}-09$ \\
\hline $2.50 \mathrm{e}-01$ & $1.12 \mathrm{e}-09$ \\
\hline $2.70 \mathrm{e}-01$ & $1.17 \mathrm{e}-09$ \\
\hline $2.90 \mathrm{e}-01$ & $1.22 \mathrm{e}-09$ \\
\hline $3.10 \mathrm{e}-01$ & $1.27 \mathrm{e}-09$ \\
\hline $3.30 \mathrm{e}-01$ & $1.31 \mathrm{e}-09$ \\
\hline $3.50 \mathrm{e}-01$ & $1.36 \mathrm{e}-09$ \\
\hline $3.70 \mathrm{e}-01$ & $1.41 \mathrm{e}-09$ \\
\hline $3.90 \mathrm{e}-01$ & $1.48 \mathrm{e}-09$ \\
\hline $4.20 \mathrm{e}-01$ & $1.61 \mathrm{e}-09$ \\
\hline $4.60 \mathrm{e}-01$ & $1.63 \mathrm{e}-09$ \\
\hline
\end{tabular}




\begin{tabular}{|c|c|}
\hline Neutron energy & fluence-to-KERMA conversion factor \\
\hline$[\mathrm{MeV}]$ & {$\left[\mathrm{rad} \cdot \mathrm{cm}^{2} / \mathrm{n}\right]$} \\
\hline $5.00 \mathrm{e}-01$ & $1.58 \mathrm{e}-09$ \\
\hline $5.40 \mathrm{e}-01$ & $1.64 \mathrm{e}-09$ \\
\hline $5.80 \mathrm{e}-01$ & $1.70 \mathrm{e}-09$ \\
\hline $6.20 \mathrm{e}-01$ & $1.76 \mathrm{e}-09$ \\
\hline $6.60 \mathrm{e}-01$ & $1.82 \mathrm{e}-09$ \\
\hline $7.00 \mathrm{e}-01$ & $1.87 \mathrm{e}-09$ \\
\hline $7.40 \mathrm{e}-01$ & $1.92 \mathrm{e}-09$ \\
\hline $7.80 \mathrm{e}-01$ & $1.98 \mathrm{e}-09$ \\
\hline $8.20 \mathrm{e}-01$ & $2.03 \mathrm{e}-09$ \\
\hline $8.60 \mathrm{e}-01$ & $2.08 \mathrm{e}-09$ \\
\hline $9.00 \mathrm{e}-01$ & $2.15 \mathrm{e}-09$ \\
\hline $9.40 \mathrm{e}-01$ & $2.25 \mathrm{e}-09$ \\
\hline $9.80 \mathrm{e}-01$ & $2.44 \mathrm{e}-09$ \\
\hline $1.05 \mathrm{e}+00$ & $2.47 \mathrm{e}-09$ \\
\hline $1.15 \mathrm{e}+00$ & $2.44 \mathrm{e}-09$ \\
\hline $1.25 \mathrm{e}+00$ & $2.54 \mathrm{e}-09$ \\
\hline $1.35 \mathrm{e}+00$ & $2.62 \mathrm{e}-09$ \\
\hline $1.45 \mathrm{e}+00$ & $2.67 \mathrm{e}-09$ \\
\hline $1.55 \mathrm{e}+00$ & $2.74 \mathrm{e}-09$ \\
\hline $1.65 \mathrm{e}+00$ & $2.85 \mathrm{e}-09$ \\
\hline $1.75 \mathrm{e}+00$ & $2.88 \mathrm{e}-09$ \\
\hline $1.85 \mathrm{e}+00$ & $3.00 \mathrm{e}-09$ \\
\hline $1.95 \mathrm{e}+00$ & $3.02 \mathrm{e}-09$ \\
\hline $2.10 \mathrm{e}+00$ & $3.10 \mathrm{e}-09$ \\
\hline $2.30 \mathrm{e}+00$ & $3.15 \mathrm{e}-09$ \\
\hline $2.50 \mathrm{e}+00$ & $3.27 \mathrm{e}-09$ \\
\hline $2.70 \mathrm{e}+00$ & $3.42 \mathrm{e}-09$ \\
\hline $2.90 \mathrm{e}+00$ & $3.55 \mathrm{e}-09$ \\
\hline $2.30 \mathrm{e}+01$ & $7.34 \mathrm{e}-09$ \\
\hline $3.10 \mathrm{e}+00$ & $3.69 \mathrm{e}-09$ \\
\hline $3.30 \mathrm{e}+00$ & $4.02 \mathrm{e}-09$ \\
\hline $3.50 \mathrm{e}+00$ & $4.10 \mathrm{e}-09$ \\
\hline $3.70 \mathrm{e}+00$ & $4.20 \mathrm{e}-09$ \\
\hline $3.90 \mathrm{e}+00$ & $4.13 \mathrm{e}-09$ \\
\hline $4.20 \mathrm{e}+00$ & $4.26 \mathrm{e}-09$ \\
\hline $4.60 \mathrm{e}+00$ & $4.26 \mathrm{e}-09$ \\
\hline $5.00 \mathrm{e}+00$ & $4.51 \mathrm{e}-09$ \\
\hline $5.40 \mathrm{e}+00$ & $4.39 \mathrm{e}-09$ \\
\hline $5.80 \mathrm{e}+00$ & $4.60 \mathrm{e}-09$ \\
\hline $6.20 \mathrm{e}+00$ & $4.71 \mathrm{e}-09$ \\
\hline $6.60 \mathrm{e}+00$ & $4.84 \mathrm{e}-09$ \\
\hline
\end{tabular}




\begin{tabular}{|c|c|}
\hline Neutron energy & fluence-to-KERMA conversion factor \\
\hline$[\mathrm{MeV}]$ & {$\left[\mathrm{rad} \cdot \mathrm{cm}^{2} / \mathrm{n}\right]$} \\
\hline \hline $7.00 \mathrm{e}+00$ & $5.06 \mathrm{e}-09$ \\
\hline $7.40 \mathrm{e}+00$ & $5.33 \mathrm{e}-09$ \\
\hline $7.80 \mathrm{e}+00$ & $5.24 \mathrm{e}-09$ \\
\hline $8.20 \mathrm{e}+00$ & $5.20 \mathrm{e}-09$ \\
\hline $8.60 \mathrm{e}+00$ & $5.38 \mathrm{e}-09$ \\
\hline $9.00 \mathrm{e}+00$ & $5.46 \mathrm{e}-09$ \\
\hline $9.40 \mathrm{e}+00$ & $5.50 \mathrm{e}-09$ \\
\hline $9.80 \mathrm{e}+00$ & $5.64 \mathrm{e}-09$ \\
\hline $1.05 \mathrm{e}+01$ & $5.78 \mathrm{e}-09$ \\
\hline $1.15 \mathrm{e}+01$ & $6.20 \mathrm{e}-09$ \\
\hline $1.25 \mathrm{e}+01$ & $6.17 \mathrm{e}-09$ \\
\hline $1.35 \mathrm{e}+01$ & $6.41 \mathrm{e}-09$ \\
\hline $1.45 \mathrm{e}+01$ & $6.66 \mathrm{e}-09$ \\
\hline $1.55 \mathrm{e}+01$ & $6.81 \mathrm{e}-09$ \\
\hline $1.65 \mathrm{e}+01$ & $6.90 \mathrm{e}-09$ \\
\hline $1.75 \mathrm{e}+01$ & $6.99 \mathrm{e}-09$ \\
\hline $1.85 \mathrm{e}+01$ & $7.10 \mathrm{e}-09$ \\
\hline $1.95 \mathrm{e}+01$ & $7.23 \mathrm{e}-09$ \\
\hline $2.10 \mathrm{e}+01$ & $7.37 \mathrm{e}-09$ \\
\hline $2.50 \mathrm{e}+01$ & $7.28 \mathrm{e}-09$ \\
\hline $2.70 \mathrm{e}+01$ & $7.31 \mathrm{e}-09$ \\
\hline $2.90 \mathrm{e}+01$ & $7.17 \mathrm{e}-09$ \\
\hline
\end{tabular}




\section{B.2 Fluence-to-KERMA conversion factors for neutrons in natural boron, taken from Caswell et al. [16].}

\begin{tabular}{|c|c|}
\hline Neutron energy & fluence-to-KERMA conversion factor \\
\hline [MeV] & {$\left[\mathrm{rad} \cdot \mathrm{cm}^{2} / \mathrm{n}\right]$} \\
\hline $2.53 \mathrm{e}-08$ & $1.59 \mathrm{e}-06$ \\
\hline $3.60 \mathrm{e}-08$ & $1.35 \mathrm{e}-06$ \\
\hline $6.30 \mathrm{e}-08$ & $1.02 \mathrm{e}-06$ \\
\hline $1.10 \mathrm{e}-07$ & $7.70 \mathrm{e}-07$ \\
\hline $2.00 \mathrm{e}-07$ & $5.72 \mathrm{e}-07$ \\
\hline $3.60 \mathrm{e}-07$ & $4.26 \mathrm{e}-07$ \\
\hline $6.30 \mathrm{e}-07$ & $3.21 \mathrm{e}-07$ \\
\hline $1.10 \mathrm{e}-06$ & $2.43 \mathrm{e}-07$ \\
\hline $2.00 \mathrm{e}-06$ & $1.81 \mathrm{e}-07$ \\
\hline $3.60 \mathrm{e}-06$ & $1.35 \mathrm{e}-07$ \\
\hline $6.30 \mathrm{e}-06$ & $1.02 \mathrm{e}-07$ \\
\hline $1.10 \mathrm{e}-05$ & $7.68 \mathrm{e}-08$ \\
\hline $2.00 \mathrm{e}-05$ & $5.70 \mathrm{e}-08$ \\
\hline $3.60 \mathrm{e}-05$ & $4.24 \mathrm{e}-08$ \\
\hline $6.30 \mathrm{e}-05$ & $3.20 \mathrm{e}-08$ \\
\hline $1.10 \mathrm{e}-04$ & $2.42 \mathrm{e}-08$ \\
\hline $2.00 \mathrm{e}-04$ & $1.79 \mathrm{e}-08$ \\
\hline $3.60 \mathrm{e}-04$ & $1.33 \mathrm{e}-08$ \\
\hline $6.30 \mathrm{e}-04$ & $1.00 \mathrm{e}-08$ \\
\hline $1.10 \mathrm{e}-03$ & $7.56 \mathrm{e}-09$ \\
\hline $2.00 \mathrm{e}-03$ & $5.59 \mathrm{e}-09$ \\
\hline $3.60 \mathrm{e}-03$ & $4.15 \mathrm{e}-09$ \\
\hline $6.30 \mathrm{e}-03$ & $3.13 \mathrm{e}-09$ \\
\hline $1.10 \mathrm{e}-02$ & $2.37 \mathrm{e}-09$ \\
\hline $2.00 \mathrm{e}-02$ & $1.78 \mathrm{e}-09$ \\
\hline $3.60 \mathrm{e}-02$ & $1.36 \mathrm{e}-09$ \\
\hline $6.30 \mathrm{e}-02$ & $1.08 \mathrm{e}-09$ \\
\hline $8.20 \mathrm{e}-02$ & $9.68 \mathrm{e}-10$ \\
\hline $8.60 \mathrm{e}-02$ & $9.52 \mathrm{e}-10$ \\
\hline $9.00 \mathrm{e}-02$ & $9.38 \mathrm{e}-10$ \\
\hline $9.40 \mathrm{e}-02$ & $9.24 \mathrm{e}-10$ \\
\hline $9.80 \mathrm{e}-02$ & $9.11 \mathrm{e}-10$ \\
\hline
\end{tabular}




\begin{tabular}{|c|c|}
\hline Neutron energy & fluence-to-KERMA conversion factor \\
\hline$[\mathrm{MeV}]$ & {$\left[\mathrm{rad} \cdot \mathrm{cm}^{2} / \mathrm{n}\right]$} \\
\hline $1.05 \mathrm{e}-01$ & $8.91 \mathrm{e}-10$ \\
\hline $1.15 \mathrm{e}-01$ & $8.65 \mathrm{e}-10$ \\
\hline $1.25 \mathrm{e}-01$ & $8.42 \mathrm{e}-10$ \\
\hline $1.35 \mathrm{e}-01$ & $8.22 \mathrm{e}-10$ \\
\hline $1.45 \mathrm{e}-01$ & $8.04 \mathrm{e}-10$ \\
\hline $1.55 \mathrm{e}-01$ & $7.87 \mathrm{e}-10$ \\
\hline $1.65 \mathrm{e}-01$ & $7.72 \mathrm{e}-10$ \\
\hline $1.75 \mathrm{e}-01$ & $7.58 \mathrm{e}-10$ \\
\hline $1.85 \mathrm{e}-01$ & $7.45 \mathrm{e}-10$ \\
\hline $1.95 \mathrm{e}-01$ & $7.33 \mathrm{e}-10$ \\
\hline $2.10 \mathrm{e}-01$ & $7.17 \mathrm{e}-10$ \\
\hline $2.30 \mathrm{e}-01$ & $6.97 \mathrm{e}-10$ \\
\hline $2.50 \mathrm{e}-01$ & $6.78 \mathrm{e}-10$ \\
\hline $2.70 \mathrm{e}-01$ & $6.59 \mathrm{e}-10$ \\
\hline $2.90 \mathrm{e}-01$ & $6.42 \mathrm{e}-10$ \\
\hline $3.10 \mathrm{e}-01$ & $6.27 \mathrm{e}-10$ \\
\hline $3.30 \mathrm{e}-01$ & $6.16 \mathrm{e}-10$ \\
\hline $3.50 \mathrm{e}-01$ & $6.08 \mathrm{e}-10$ \\
\hline $3.70 \mathrm{e}-01$ & $6.06 \mathrm{e}-10$ \\
\hline $3.90 \mathrm{e}-01$ & $6.18 \mathrm{e}-10$ \\
\hline $4.20 \mathrm{e}-01$ & $7.08 \mathrm{e}-10$ \\
\hline $4.60 \mathrm{e}-01$ & $6.93 \mathrm{e}-10$ \\
\hline $5.00 \mathrm{e}-01$ & $6.25 \mathrm{e}-10$ \\
\hline $5.40 \mathrm{e}-01$ & $6.86 \mathrm{e}-10$ \\
\hline $5.80 \mathrm{e}-01$ & $5.46 \mathrm{e}-10$ \\
\hline $6.20 \mathrm{e}-01$ & $5.11 \mathrm{e}-10$ \\
\hline $6.60 \mathrm{e}-01$ & $4.82 \mathrm{e}-10$ \\
\hline $7.00 \mathrm{e}-01$ & $4.61 \mathrm{e}-10$ \\
\hline $7.40 \mathrm{e}-01$ & $4.47 \mathrm{e}-10$ \\
\hline $7.80 \mathrm{e}-01$ & $4.38 \mathrm{e}-10$ \\
\hline $8.20 \mathrm{e}-01$ & $4.33 \mathrm{e}-10$ \\
\hline $8.60 \mathrm{e}-01$ & $4.29 \mathrm{e}-10$ \\
\hline $9.00 \mathrm{e}-01$ & $4.27 \mathrm{e}-10$ \\
\hline $9.40 \mathrm{e}-01$ & $4.28 \mathrm{e}-10$ \\
\hline $9.80 \mathrm{e}-01$ & $4.26 \mathrm{e}-10$ \\
\hline $1.05 \mathrm{e}+00$ & $4.34 \mathrm{e}-10$ \\
\hline $1.15 \mathrm{e}+00$ & $4.82 \mathrm{e}-10$ \\
\hline $1.25 \mathrm{e}+00$ & $6.58 \mathrm{e}-10$ \\
\hline $1.35 \mathrm{e}+00$ & $6.62 \mathrm{e}-10$ \\
\hline $1.45 \mathrm{e}+00$ & $6.16 \mathrm{e}-10$ \\
\hline $1.55 \mathrm{e}+00$ & $6.45 \mathrm{e}-10$ \\
\hline
\end{tabular}




\begin{tabular}{|c|c|}
\hline Neutron energy & fluence-to-KERMA conversion factor \\
\hline$[\mathrm{MeV}]$ & {$\left[\mathrm{rad} \cdot \mathrm{cm}^{2} / \mathrm{n}\right]$} \\
\hline $1.65 \mathrm{e}+00$ & $7.20 \mathrm{e}-10$ \\
\hline $1.75 \mathrm{e}+00$ & $8.66 \mathrm{e}-10$ \\
\hline $1.85 \mathrm{e}+00$ & $9.08 \mathrm{e}-10$ \\
\hline $1.95 \mathrm{e}+00$ & $8.70 \mathrm{e}-10$ \\
\hline $2.10 \mathrm{e}+00$ & $8.21 \mathrm{e}-10$ \\
\hline $2.30 \mathrm{e}+00$ & $7.45 \mathrm{e}-10$ \\
\hline $2.50 \mathrm{e}+00$ & $8.97 \mathrm{e}-10$ \\
\hline $2.70 \mathrm{e}+00$ & $9.34 \mathrm{e}-10$ \\
\hline $2.90 \mathrm{e}+00$ & $9.23 \mathrm{e}-10$ \\
\hline $3.10 \mathrm{e}+00$ & $8.76 \mathrm{e}-10$ \\
\hline $3.30 \mathrm{e}+00$ & $8.61 \mathrm{e}-10$ \\
\hline $3.50 \mathrm{e}+00$ & $9.39 \mathrm{e}-10$ \\
\hline $3.70 \mathrm{e}+00$ & $9.86 \mathrm{e}-10$ \\
\hline $3.90 \mathrm{e}+00$ & $1.01 \mathrm{e}-09$ \\
\hline $4.20 \mathrm{e}+00$ & $1.06 \mathrm{e}-09$ \\
\hline $4.60 \mathrm{e}+00$ & $1.24 \mathrm{e}-09$ \\
\hline $5.00 \mathrm{e}+00$ & $1.31 \mathrm{e}-09$ \\
\hline $5.40 \mathrm{e}+00$ & $1.31 \mathrm{e}-09$ \\
\hline $5.80 \mathrm{e}+00$ & $1.36 \mathrm{e}-09$ \\
\hline $6.20 \mathrm{e}+00$ & $1.38 \mathrm{e}-09$ \\
\hline $6.60 \mathrm{e}+00$ & $1.31 \mathrm{e}-09$ \\
\hline $7.00 \mathrm{e}+00$ & $1.30 \mathrm{e}-09$ \\
\hline $7.40 \mathrm{e}+00$ & $1.27 \mathrm{e}-09$ \\
\hline $7.80 \mathrm{e}+00$ & $1.35 \mathrm{e}-09$ \\
\hline $8.20 \mathrm{e}+00$ & $1.28 \mathrm{e}-09$ \\
\hline $8.60 \mathrm{e}+00$ & $1.29 \mathrm{e}-09$ \\
\hline $9.00 \mathrm{e}+00$ & $1.30 \mathrm{e}-09$ \\
\hline $9.40 \mathrm{e}+00$ & $1.32 \mathrm{e}-09$ \\
\hline $9.80 \mathrm{e}+00$ & $1.41 \mathrm{e}-09$ \\
\hline $1.05 e+01$ & $1.46 \mathrm{e}-09$ \\
\hline $1.15 e+01$ & $1.57 \mathrm{e}-09$ \\
\hline $1.25 \mathrm{e}+01$ & $1.71 \mathrm{e}-09$ \\
\hline $1.35 \mathrm{e}+01$ & $1.86 \mathrm{e}-09$ \\
\hline $1.45 \mathrm{e}+01$ & $2.00 \mathrm{e}-09$ \\
\hline $1.55 \mathrm{e}+01$ & $2.08 \mathrm{e}-09$ \\
\hline $1.65 \mathrm{e}+01$ & $2.17 \mathrm{e}-09$ \\
\hline $1.75 \mathrm{e}+01$ & $2.23 \mathrm{e}-09$ \\
\hline $1.85 e+01$ & $2.28 \mathrm{e}-09$ \\
\hline $1.95 \mathrm{e}+01$ & $2.31 \mathrm{e}-09$ \\
\hline $2.10 \mathrm{e}+01$ & $2.45 \mathrm{e}-09$ \\
\hline $2.30 \mathrm{e}+01$ & $2.55 \mathrm{e}-09$ \\
\hline
\end{tabular}




\begin{tabular}{|c|c|}
\hline Neutron energy & fluence-to-KERMA conversion factor \\
\hline$[\mathrm{MeV}]$ & {$\left[\mathrm{rad} \cdot \mathrm{cm}^{2} / \mathrm{n}\right]$} \\
\hline \hline $2.50 \mathrm{e}+01$ & $2.65 \mathrm{e}-09$ \\
\hline $2.70 \mathrm{e}+01$ & $2.73 \mathrm{e}-09$ \\
\hline $2.90 \mathrm{e}+01$ & $2.85 \mathrm{e}-09$ \\
\hline
\end{tabular}




\section{B.3 Photon mass attenuation coefficients in tissues, taken} from the International Commission on Radiation Units and Measurements (ICRU) Report No. 46 [14].

\begin{tabular}{|c|c|}
\hline Photon energy & photon mass attenuation coefficient \\
\hline$[\mathrm{MeV}]$ & {$\left[\mathrm{m}^{2} / \mathrm{kg}\right]$} \\
\hline \hline $1.00 \mathrm{e}-02$ & $4.47 \mathrm{e}-01$ \\
\hline $1.50 \mathrm{e}-02$ & $1.23 \mathrm{e}-01$ \\
\hline $2.00 \mathrm{e}-02$ & $4.94 \mathrm{e}-02$ \\
\hline $3.00 \mathrm{e}-02$ & $1.40 \mathrm{e}-02$ \\
\hline $4.00 \mathrm{e}-02$ & $6.34 \mathrm{e}-03$ \\
\hline $5.00 \mathrm{e}-02$ & $3.92 \mathrm{e}-03$ \\
\hline $6.00 \mathrm{e}-02$ & $3.02 \mathrm{e}-03$ \\
\hline $8.00 \mathrm{e}-02$ & $2.52 \mathrm{e}-03$ \\
\hline $1.00 \mathrm{e}-01$ & $2.49 \mathrm{e}-03$ \\
\hline $1.50 \mathrm{e}-01$ & $2.73 \mathrm{e}-03$ \\
\hline $2.00 \mathrm{e}-01$ & $2.94 \mathrm{e}-03$ \\
\hline $3.00 \mathrm{e}-01$ & $3.16 \mathrm{e}-03$ \\
\hline $4.00 \mathrm{e}-01$ & $3.25 \mathrm{e}-03$ \\
\hline $5.00 \mathrm{e}-01$ & $3.27 \mathrm{e}-03$ \\
\hline $6.00 \mathrm{e}-01$ & $3.25 \mathrm{e}-03$ \\
\hline $8.00 \mathrm{e}-01$ & $3.17 \mathrm{e}-03$ \\
\hline $1.00 \mathrm{e}+00$ & $3.07 \mathrm{e}-03$ \\
\hline $1.50 \mathrm{e}+00$ & $2.80 \mathrm{e}-03$ \\
\hline $2.00 \mathrm{e}+00$ & $2.58 \mathrm{e}-03$ \\
\hline $3.00 \mathrm{e}+00$ & $2.25 \mathrm{e}-03$ \\
\hline $4.00 \mathrm{e}+00$ & $2.04 \mathrm{e}-03$ \\
\hline $5.00 \mathrm{e}+00$ & $1.89 \mathrm{e}-03$ \\
\hline $6.00 \mathrm{e}+00$ & $1.78 \mathrm{e}-03$ \\
\hline $8.00 \mathrm{e}+00$ & $1.64 \mathrm{e}-03$ \\
\hline $1.00 \mathrm{e}+01$ & $1.54 \mathrm{e}-03$ \\
\hline $1.50 \mathrm{e}+01$ & $1.42 \mathrm{e}-03$ \\
\hline $2.00 \mathrm{e}+01$ & $1.36 \mathrm{e}-03$ \\
\hline $3.00 \mathrm{e}+01$ & $1.30 \mathrm{e}-03$ \\
\hline $4.00 \mathrm{e}+01$ & $1.28 \mathrm{e}-03$ \\
\hline $5.00 \mathrm{e}+01$ & $1.26 \mathrm{e}-03$ \\
\hline $6.00 \mathrm{e}+01$ & $1.24 \mathrm{e}-03$ \\
\hline $8.00 \mathrm{e}+01$ & $1.21 \mathrm{e}-03$ \\
\hline $1.00 \mathrm{e}+02$ & $1.19 \mathrm{e}-03$ \\
\hline
\end{tabular}




\section{B.4 Fluence-to-KERMA conversion factors for neutrons in tissues, taken from Zamenhof et al. [15].}

\begin{tabular}{|c|c|}
\hline Neutron energy & fluence-to-KERMA conversion factor \\
\hline$[\mathrm{MeV}]$ & {$\left[\mathrm{rad} \cdot \mathrm{cm}^{2} / \mathrm{n}\right]$} \\
\hline$\overline{1 \mathrm{e}-10}$ & $2.3717 \mathrm{e}-10$ \\
\hline $2.50 \mathrm{e}-08$ & $1.50 \mathrm{e}-11$ \\
\hline $3.50 \mathrm{e}-08$ & $1.26 \mathrm{e}-11$ \\
\hline $4.50 \mathrm{e}-08$ & $1.12 \mathrm{e}-11$ \\
\hline $5.50 \mathrm{e}-08$ & $1.02 \mathrm{e}-11$ \\
\hline $6.50 \mathrm{e}-08$ & $9.37 \mathrm{e}-12$ \\
\hline $7.50 \mathrm{e}-08$ & $8.73 \mathrm{e}-12$ \\
\hline $8.50 \mathrm{e}-08$ & $8.20 \mathrm{e}-12$ \\
\hline $9.50 \mathrm{e}-08$ & $7.76 \mathrm{e}-12$ \\
\hline $1.00 \mathrm{e}-07$ & $7.56 \mathrm{e}-12$ \\
\hline $2.50 \mathrm{e}-07$ & $4.79 \mathrm{e}-12$ \\
\hline $3.00 \mathrm{e}-07$ & $4.37 \mathrm{e}-12$ \\
\hline $3.50 \mathrm{e}-07$ & $4.04 \mathrm{e}-12$ \\
\hline $4.00 \mathrm{e}-07$ & $3.78 \mathrm{e}-12$ \\
\hline $4.50 \mathrm{e}-07$ & $3.57 \mathrm{e}-12$ \\
\hline $5.00 \mathrm{e}-07$ & $3.39 \mathrm{e}-12$ \\
\hline $5.50 \mathrm{e}-07$ & $3.23 \mathrm{e}-12$ \\
\hline $6.00 \mathrm{e}-07$ & $3.10 \mathrm{e}-12$ \\
\hline $6.50 \mathrm{e}-07$ & $2.98 \mathrm{e}-12$ \\
\hline $7.00 \mathrm{e}-07$ & $2.87 \mathrm{e}-12$ \\
\hline $7.50 \mathrm{e}-07$ & $2.77 \mathrm{e}-12$ \\
\hline $8.00 \mathrm{e}-07$ & $2.68 \mathrm{e}-12$ \\
\hline $8.50 \mathrm{e}-07$ & $2.60 \mathrm{e}-12$ \\
\hline $9.00 \mathrm{e}-07$ & $2.53 \mathrm{e}-12$ \\
\hline $9.50 \mathrm{e}-07$ & $2.46 \mathrm{e}-12$ \\
\hline $1.00 \mathrm{e}-06$ & $2.40 \mathrm{e}-12$ \\
\hline $1.50 \mathrm{e}-06$ & $1.97 \mathrm{e}-12$ \\
\hline $2.00 \mathrm{e}-06$ & $1.72 \mathrm{e}-12$ \\
\hline $2.50 \mathrm{e}-06$ & $1.54 \mathrm{e}-12$ \\
\hline $3.00 \mathrm{e}-06$ & $1.40 \mathrm{e}-12$ \\
\hline $3.50 \mathrm{e}-06$ & $1.30 \mathrm{e}-12$ \\
\hline $4.00 \mathrm{e}-06$ & $1.23 \mathrm{e}-12$ \\
\hline $4.50 \mathrm{e}-06$ & $1.17 \mathrm{e}-12$ \\
\hline $5.00 \mathrm{e}-06$ & $1.12 \mathrm{e}-12$ \\
\hline $5.50 \mathrm{e}-06$ & $1.08 \mathrm{e}-12$ \\
\hline $6.00 \mathrm{e}-06$ & $1.04 \mathrm{e}-12$ \\
\hline $6.50 \mathrm{e}-06$ & $1.01 \mathrm{e}-12$ \\
\hline
\end{tabular}




\begin{tabular}{|c|c|}
\hline Neutron energy & fluence-to-KERMA conversion factor \\
\hline$[\mathrm{MeV}]$ & {$\left[\mathrm{rad} \cdot \mathrm{cm}^{2} / \mathrm{n}\right]$} \\
\hline $7.00 \mathrm{e}-06$ & $9.79 \mathrm{e}-13$ \\
\hline $7.50 \mathrm{e}-06$ & $9.54 \mathrm{e}-13$ \\
\hline $8.00 \mathrm{e}-06$ & $9.32 \mathrm{e}-13$ \\
\hline $8.50 \mathrm{e}-06$ & $9.12 \mathrm{e}-13$ \\
\hline $9.00 \mathrm{e}-06$ & $8.94 \mathrm{e}-13$ \\
\hline $9.50 \mathrm{e}-06$ & $8.78 \mathrm{e}-13$ \\
\hline $1.00 \mathrm{e}-05$ & $8.63 \mathrm{e}-13$ \\
\hline $1.50 \mathrm{e}-05$ & $7.80 \mathrm{e}-13$ \\
\hline $2.00 \mathrm{e}-05$ & $7.50 \mathrm{e}-13$ \\
\hline $2.50 \mathrm{e}-05$ & $7.54 \mathrm{e}-13$ \\
\hline $3.00 \mathrm{e}-05$ & $7.80 \mathrm{e}-13$ \\
\hline $3.50 \mathrm{e}-05$ & $8.30 \mathrm{e}-13$ \\
\hline $4.00 \mathrm{e}-05$ & $8.46 \mathrm{e}-13$ \\
\hline $4.50 \mathrm{e}-05$ & $8.62 \mathrm{e}-13$ \\
\hline $5.00 \mathrm{e}-05$ & $8.86 \mathrm{e}-13$ \\
\hline $5.50 \mathrm{e}-05$ & $9.16 \mathrm{e}-13$ \\
\hline $6.00 \mathrm{e}-05$ & $9.50 \mathrm{e}-13$ \\
\hline $6.50 \mathrm{e}-05$ & $9.88 \mathrm{e}-13$ \\
\hline $7.00 \mathrm{e}-05$ & $1.03 \mathrm{e}-12$ \\
\hline $7.50 \mathrm{e}-05$ & $1.07 \mathrm{e}-12$ \\
\hline $8.00 \mathrm{e}-05$ & $1.12 \mathrm{e}-12$ \\
\hline $8.50 \mathrm{e}-05$ & $1.16 \mathrm{e}-12$ \\
\hline $9.00 \mathrm{e}-05$ & $1.21 \mathrm{e}-12$ \\
\hline $9.50 \mathrm{e}-05$ & $1.26 \mathrm{e}-12$ \\
\hline $1.00 \mathrm{e}-04$ & $1.30 \mathrm{e}-12$ \\
\hline $1.50 \mathrm{e}-04$ & $1.79 \mathrm{e}-12$ \\
\hline $2.00 \mathrm{e}-04$ & $2.30 \mathrm{e}-12$ \\
\hline $2.50 \mathrm{e}-04$ & $2.81 \mathrm{e}-12$ \\
\hline $3.00 \mathrm{e}-04$ & $3.33 \mathrm{e}-12$ \\
\hline $3.50 \mathrm{e}-04$ & $3.85 \mathrm{e}-12$ \\
\hline $4.00 \mathrm{e}-04$ & $4.37 \mathrm{e}-12$ \\
\hline $4.50 \mathrm{e}-04$ & $4.89 \mathrm{e}-12$ \\
\hline $5.00 \mathrm{e}-04$ & $5.41 \mathrm{e}-12$ \\
\hline $5.50 \mathrm{e}-04$ & $5.94 \mathrm{e}-12$ \\
\hline $6.00 \mathrm{e}-04$ & $6.46 \mathrm{e}-12$ \\
\hline $6.50 \mathrm{e}-04$ & $6.98 \mathrm{e}-12$ \\
\hline $7.00 \mathrm{e}-04$ & $7.51 \mathrm{e}-12$ \\
\hline $7.50 \mathrm{e}-04$ & $8.03 \mathrm{e}-12$ \\
\hline $8.00 \mathrm{e}-04$ & $8.56 \mathrm{e}-12$ \\
\hline $8.50 \mathrm{e}-04$ & $9.09 \mathrm{e}-12$ \\
\hline $9.00 \mathrm{e}-04$ & $9.61 \mathrm{e}-12$ \\
\hline
\end{tabular}




\begin{tabular}{|c|c|}
\hline Neutron energy & fluence-to-KERMA conversion factor \\
\hline$[\mathrm{MeV}]$ & {$\left[\mathrm{rad} \cdot \mathrm{cm}^{2} / \mathrm{n}\right]$} \\
\hline $9.50 \mathrm{e}-04$ & $1.01 \mathrm{e}-11$ \\
\hline $1.00 \mathrm{e}-03$ & $1.07 \mathrm{e}-11$ \\
\hline $1.50 \mathrm{e}-03$ & $1.59 \mathrm{e}-11$ \\
\hline $2.00 \mathrm{e}-03$ & $2.11 \mathrm{e}-11$ \\
\hline $2.50 \mathrm{e}-03$ & $2.62 \mathrm{e}-11$ \\
\hline $3.00 \mathrm{e}-03$ & $3.14 \mathrm{e}-11$ \\
\hline $3.50 \mathrm{e}-03$ & $3.66 \mathrm{e}-11$ \\
\hline $4.00 \mathrm{e}-03$ & $4.16 \mathrm{e}-11$ \\
\hline $4.50 \mathrm{e}-03$ & $4.66 \mathrm{e}-11$ \\
\hline $5.00 \mathrm{e}-03$ & $5.17 \mathrm{e}-11$ \\
\hline $5.50 \mathrm{e}-03$ & $5.66 \mathrm{e}-11$ \\
\hline $6.00 \mathrm{e}-03$ & $6.16 \mathrm{e}-11$ \\
\hline $6.50 \mathrm{e}-03$ & $6.65 \mathrm{e}-11$ \\
\hline $7.00 \mathrm{e}-03$ & $7.13 \mathrm{e}-11$ \\
\hline $7.50 \mathrm{e}-03$ & $7.61 \mathrm{e}-11$ \\
\hline $8.00 \mathrm{e}-03$ & $8.08 \mathrm{e}-11$ \\
\hline $8.50 \mathrm{e}-03$ & $8.56 \mathrm{e}-11$ \\
\hline $9.00 \mathrm{e}-03$ & $9.03 \mathrm{e}-11$ \\
\hline $9.50 \mathrm{e}-03$ & $9.50 \mathrm{e}-11$ \\
\hline $1.00 \mathrm{e}-02$ & $9.97 \mathrm{e}-11$ \\
\hline $1.50 \mathrm{e}-02$ & $1.45 \mathrm{e}-10$ \\
\hline $2.00 \mathrm{e}-02$ & $1.90 \mathrm{e}-10$ \\
\hline $2.50 \mathrm{e}-02$ & $2.30 \mathrm{e}-10$ \\
\hline $3.00 \mathrm{e}-02$ & $2.69 \mathrm{e}-10$ \\
\hline $3.50 \mathrm{e}-02$ & $3.06 \mathrm{e}-10$ \\
\hline $4.50 \mathrm{e}-02$ & $3.74 \mathrm{e}-10$ \\
\hline $5.50 \mathrm{e}-02$ & $4.38 \mathrm{e}-10$ \\
\hline $6.50 \mathrm{e}-02$ & $4.98 \mathrm{e}-10$ \\
\hline $7.50 \mathrm{e}-02$ & $5.52 \mathrm{e}-10$ \\
\hline $8.50 \mathrm{e}-02$ & $6.04 \mathrm{e}-10$ \\
\hline $9.50 \mathrm{e}-02$ & $6.52 \mathrm{e}-10$ \\
\hline $1.00 \mathrm{e}-01$ & $6.74 \mathrm{e}-10$ \\
\hline $1.50 \mathrm{e}-01$ & $8.74 \mathrm{e}-10$ \\
\hline $2.00 \mathrm{e}-01$ & $1.03 \mathrm{e}-09$ \\
\hline $2.50 \mathrm{e}-01$ & $1.17 \mathrm{e}-09$ \\
\hline $3.00 \mathrm{e}-01$ & $1.30 \mathrm{e}-09$ \\
\hline $3.50 \mathrm{e}-01$ & $1.42 \mathrm{e}-09$ \\
\hline $4.00 \mathrm{e}-01$ & $1.58 \mathrm{e}-09$ \\
\hline $4.50 \mathrm{e}-01$ & $1.69 \mathrm{e}-09$ \\
\hline $5.00 \mathrm{e}-01$ & $1.66 \mathrm{e}-09$ \\
\hline $5.50 \mathrm{e}-01$ & $1.73 \mathrm{e}-09$ \\
\hline
\end{tabular}




\begin{tabular}{|c|c|}
\hline Neutron energy & fluence-to-KERMA conversion factor \\
\hline$[\mathrm{MeV}]$ & {$\left[\mathrm{rad} \cdot \mathrm{cm}^{2} / \mathrm{n}\right]$} \\
\hline $6.00 \mathrm{e}-01$ & $1.81 \mathrm{e}-09$ \\
\hline $6.50 \mathrm{e}-01$ & $1.88 \mathrm{e}-09$ \\
\hline $7.00 \mathrm{e}-01$ & $1.96 \mathrm{e}-09$ \\
\hline $7.50 \mathrm{e}-01$ & $2.02 \mathrm{e}-09$ \\
\hline $8.00 \mathrm{e}-01$ & $2.09 \mathrm{e}-09$ \\
\hline $8.50 \mathrm{e}-01$ & $2.16 \mathrm{e}-09$ \\
\hline $9.00 \mathrm{e}-01$ & $2.24 \mathrm{e}-09$ \\
\hline $9.50 \mathrm{e}-01$ & $2.39 \mathrm{e}-09$ \\
\hline $1.00 \mathrm{e}+00$ & $2.54 \mathrm{e}-09$ \\
\hline $1.50 \mathrm{e}+00$ & $2.82 \mathrm{e}-09$ \\
\hline $2.00 \mathrm{e}+00$ & $3.19 \mathrm{e}-09$ \\
\hline $2.50 \mathrm{e}+00$ & $3.43 \mathrm{e}-09$ \\
\hline $3.00 \mathrm{e}+00$ & $3.79 \mathrm{e}-09$ \\
\hline $3.50 \mathrm{e}+00$ & $4.28 \mathrm{e}-09$ \\
\hline $4.00 \mathrm{e}+00$ & $4.35 \mathrm{e}-09$ \\
\hline $4.50 \mathrm{e}+00$ & $4.44 \mathrm{e}-09$ \\
\hline $5.00 \mathrm{e}+00$ & $4.70 \mathrm{e}-09$ \\
\hline $5.50 \mathrm{e}+00$ & $4.64 \mathrm{e}-09$ \\
\hline $6.50 \mathrm{e}+00$ & $5.01 \mathrm{e}-09$ \\
\hline $7.00 \mathrm{e}+00$ & $5.25 \mathrm{e}-09$ \\
\hline $7.50 \mathrm{e}+00$ & $5.50 \mathrm{e}-09$ \\
\hline $8.00 \mathrm{e}+00$ & $5.44 \mathrm{e}-09$ \\
\hline $8.50 \mathrm{e}+00$ & $5.54 \mathrm{e}-09$ \\
\hline $9.00 \mathrm{e}+00$ & $5.68 \mathrm{e}-09$ \\
\hline $9.50 \mathrm{e}+00$ & $5.76 \mathrm{e}-09$ \\
\hline $1.00 \mathrm{e}+01$ & $5.89 \mathrm{e}-09$ \\
\hline $1.05 \mathrm{e}+01$ & $5.99 \mathrm{e}-09$ \\
\hline $1.10 \mathrm{e}+01$ & $6.19 \mathrm{e}-09$ \\
\hline $1.15 \mathrm{e}+01$ & $6.41 \mathrm{e}-09$ \\
\hline $1.20 \mathrm{e}+01$ & $6.39 \mathrm{e}-09$ \\
\hline $1.25 \mathrm{e}+01$ & $6.39 \mathrm{e}-09$ \\
\hline $1.30 \mathrm{e}+01$ & $6.51 \mathrm{e}-09$ \\
\hline $1.35 \mathrm{e}+01$ & $6.63 \mathrm{e}-09$ \\
\hline $1.40 \mathrm{e}+01$ & $6.75 \mathrm{e}-09$ \\
\hline $1.45 \mathrm{e}+01$ & $6.88 \mathrm{e}-09$ \\
\hline $1.50 \mathrm{e}+01$ & $6.96 \mathrm{e}-09$ \\
\hline $1.55 \mathrm{e}+01$ & $7.06 \mathrm{e}-09$ \\
\hline $2.90 \mathrm{e}+01$ & $7.17 \mathrm{e}-09$ \\
\hline
\end{tabular}




\section{Appendix C}

\section{Simulations with the MIRD 5 anthropomorphic model}

\section{C.1 MCNP input file corresponding to the MIRD 5 phantom}

head shown in Fig. 3.1a and the BSA used in Sec. 4.8.3.

- Dose calculation in Mird4 phantom due to the neutron beam 5/19/98 -

c The design was rotated of an angle of 52.65 degree in order to have an

$c$ irradiation of the brain tumors with a minimum shower effect.

c - - - - - - - - - -

c Cells of the phantom

c

$10((1:-15: 16)(5051: 15:-60) \quad$ \$ void region of interest \#47185 186 (-16:110:111 112))

$(-299(200:-245: 203: 249))$

$2 \quad \begin{array}{lllllllllll}6 & -1 & -1 & 15 & -64 & 125 & 130 & 170 & 171 & \text { \$ segment } 1 \text { trunk }\end{array}$ \#38 \#45 \#46

$(153:-157: 152)(155: 157)(150:-152)$

$\begin{array}{llllllllllll}3 & 6 & -1 & -1 & 64 & -65 & 130 & 170 & 171 & 80 & 81 & \text { \$ segment } 2 \text { trunk }\end{array}$ \#38 \#66

$(160:-157)(161: 158)(-14: 13)$

$(71:-136: 137:-138: 139)$

$(150: 143)(140:-142: 143)(145:-147: 148)$

$4 \quad 6-1-165-66131301808081 \quad$ \$ segment 3 trunk \#5 \#69 \#66 (-122 : 120 121)

$(160:-157)(161: 158)$ $\begin{array}{lllllllllllll}5 & 7 & -1.4862 & -2 & 3 & 17 & -18 & :-2 & 3 & 25 & -26 & \text { \$ribcage segment } 3 \\ & :-2 & 3 & 27 & -66\end{array}$ 


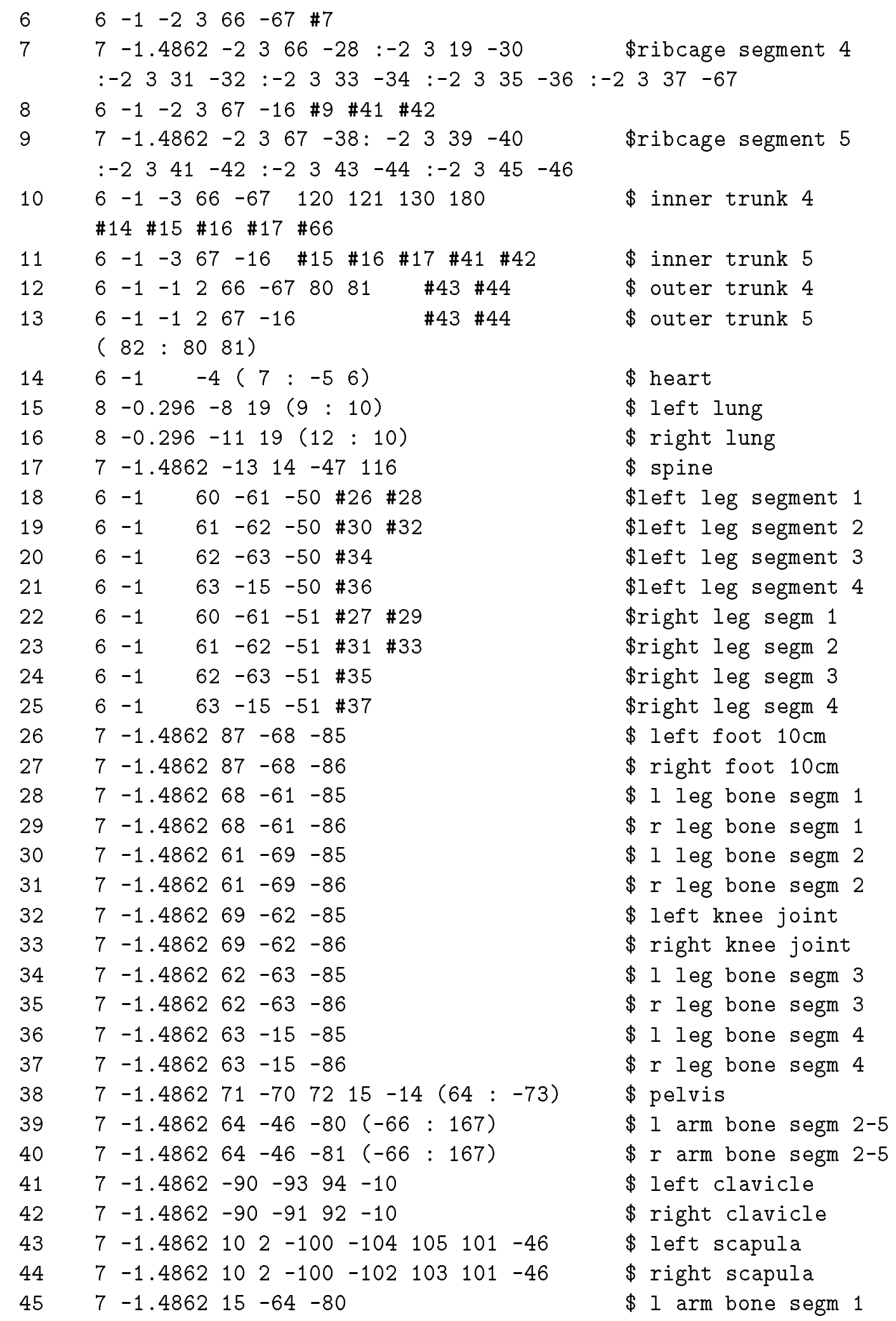




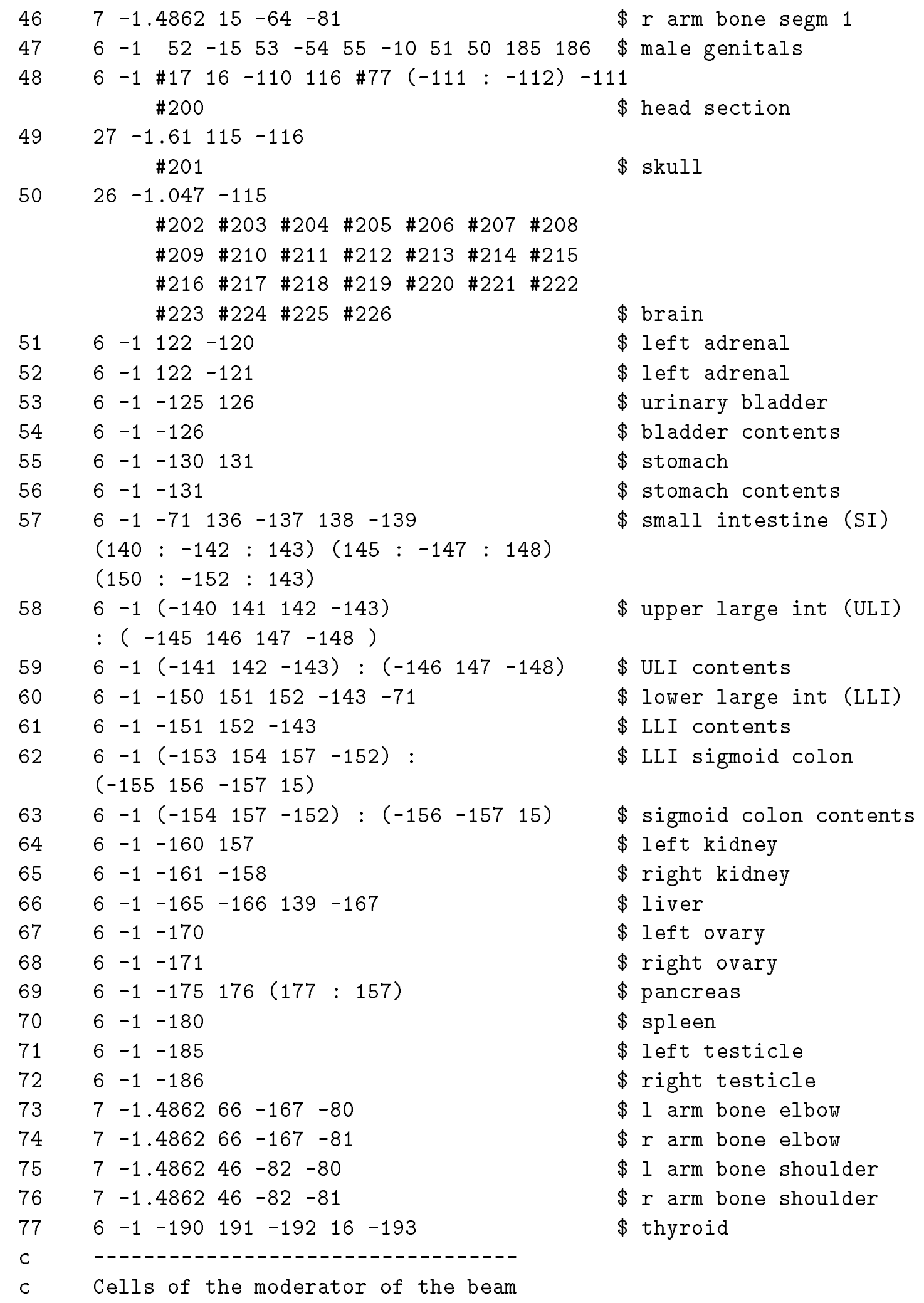




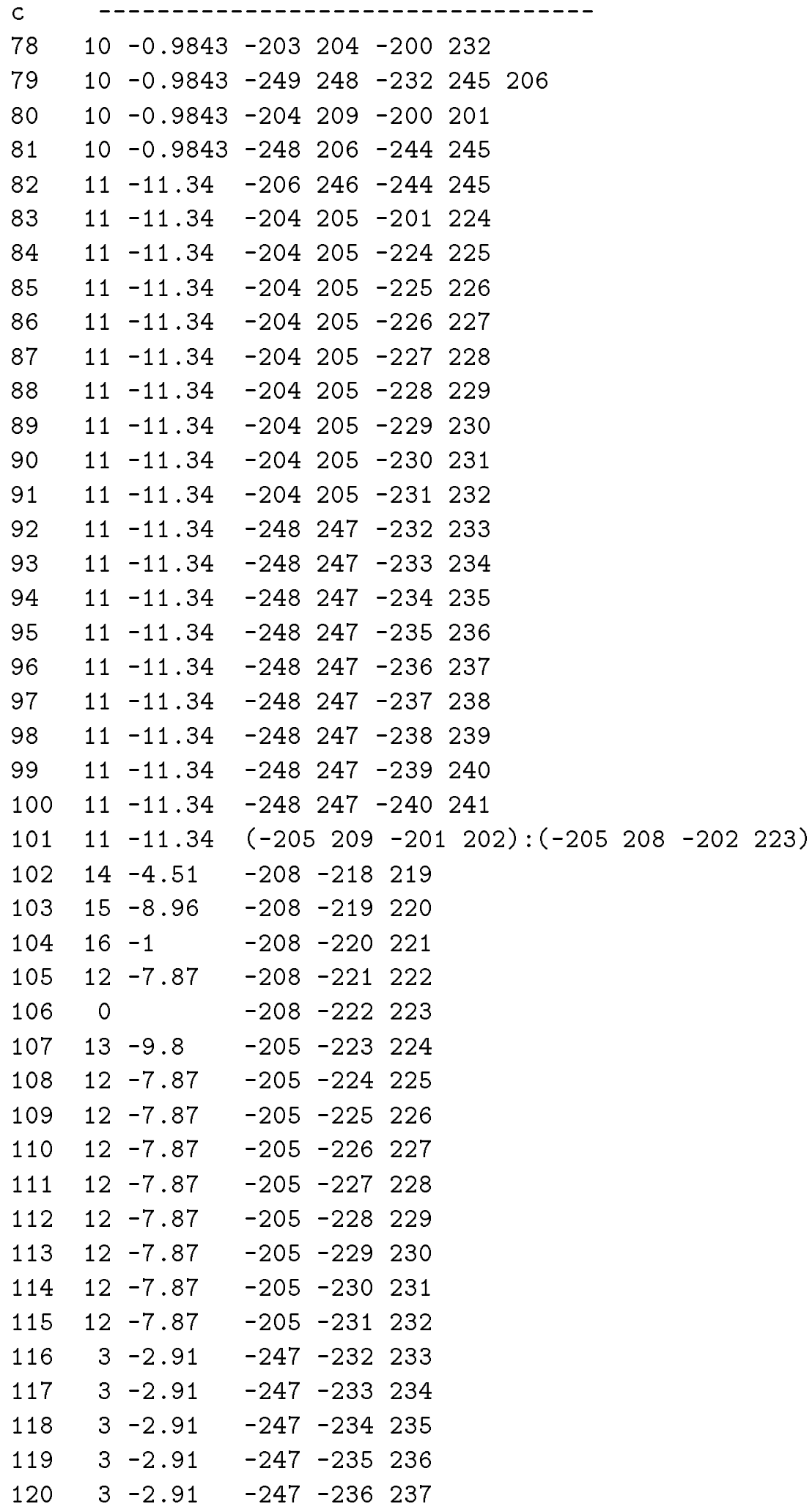




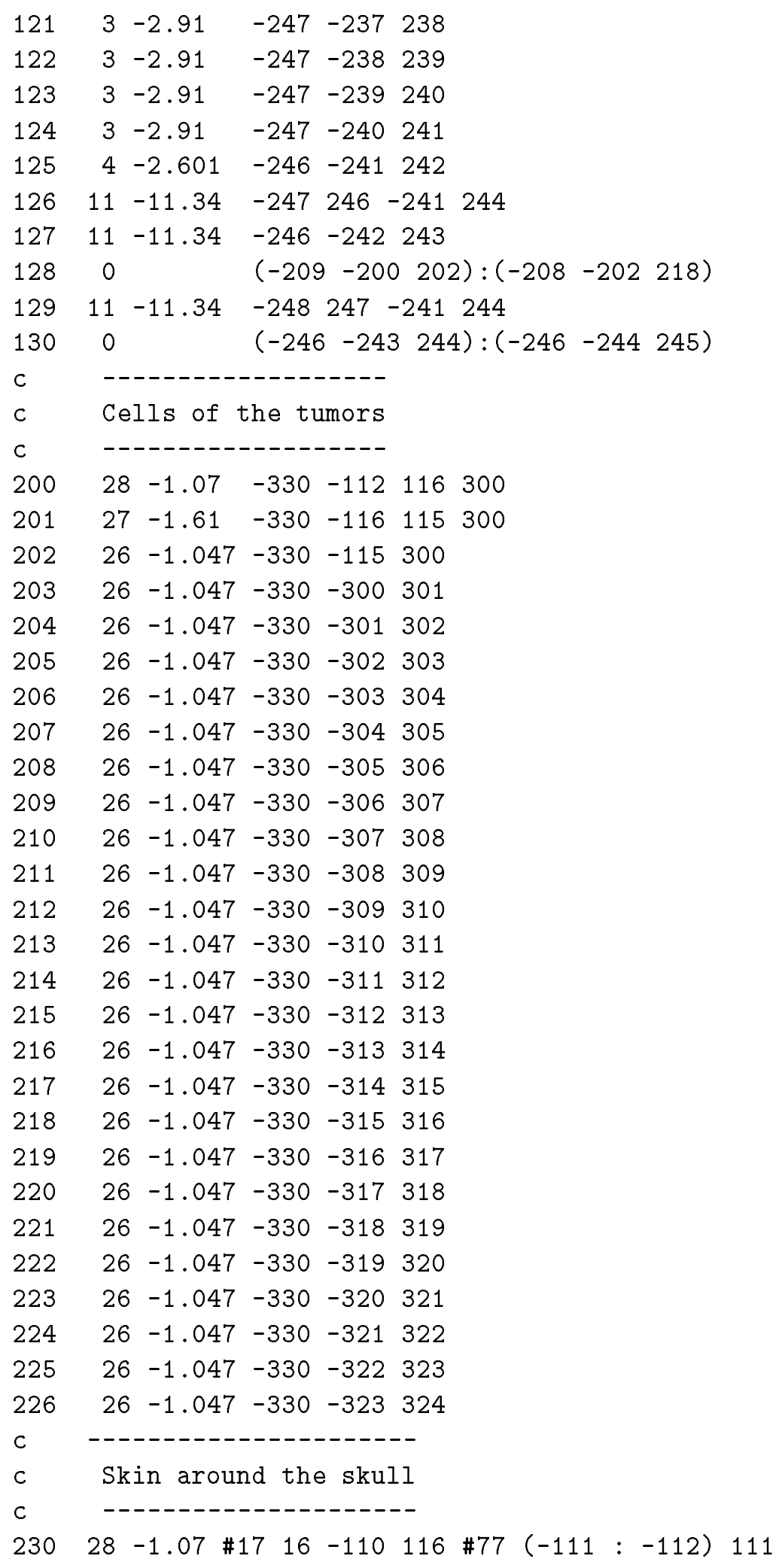




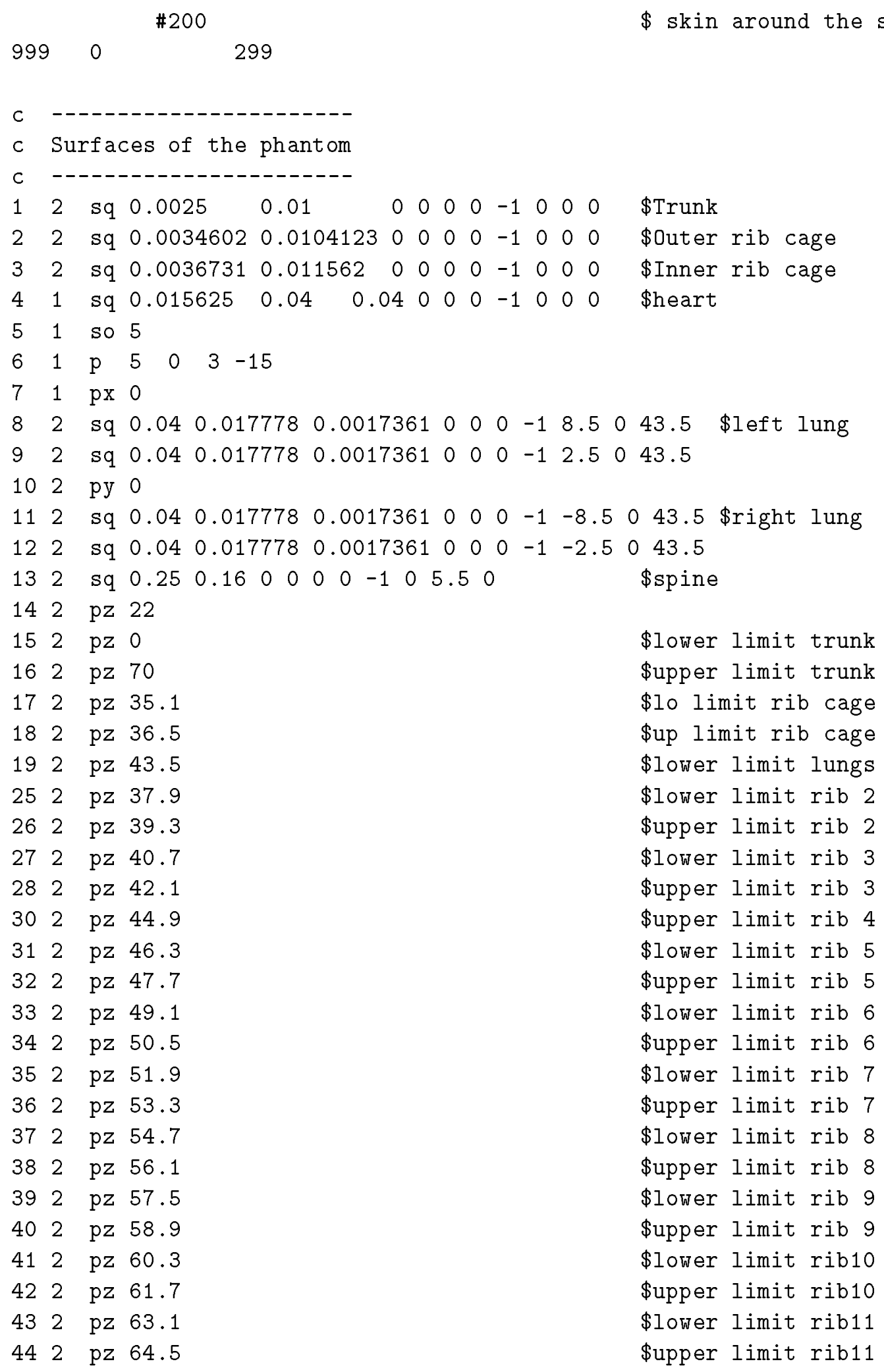




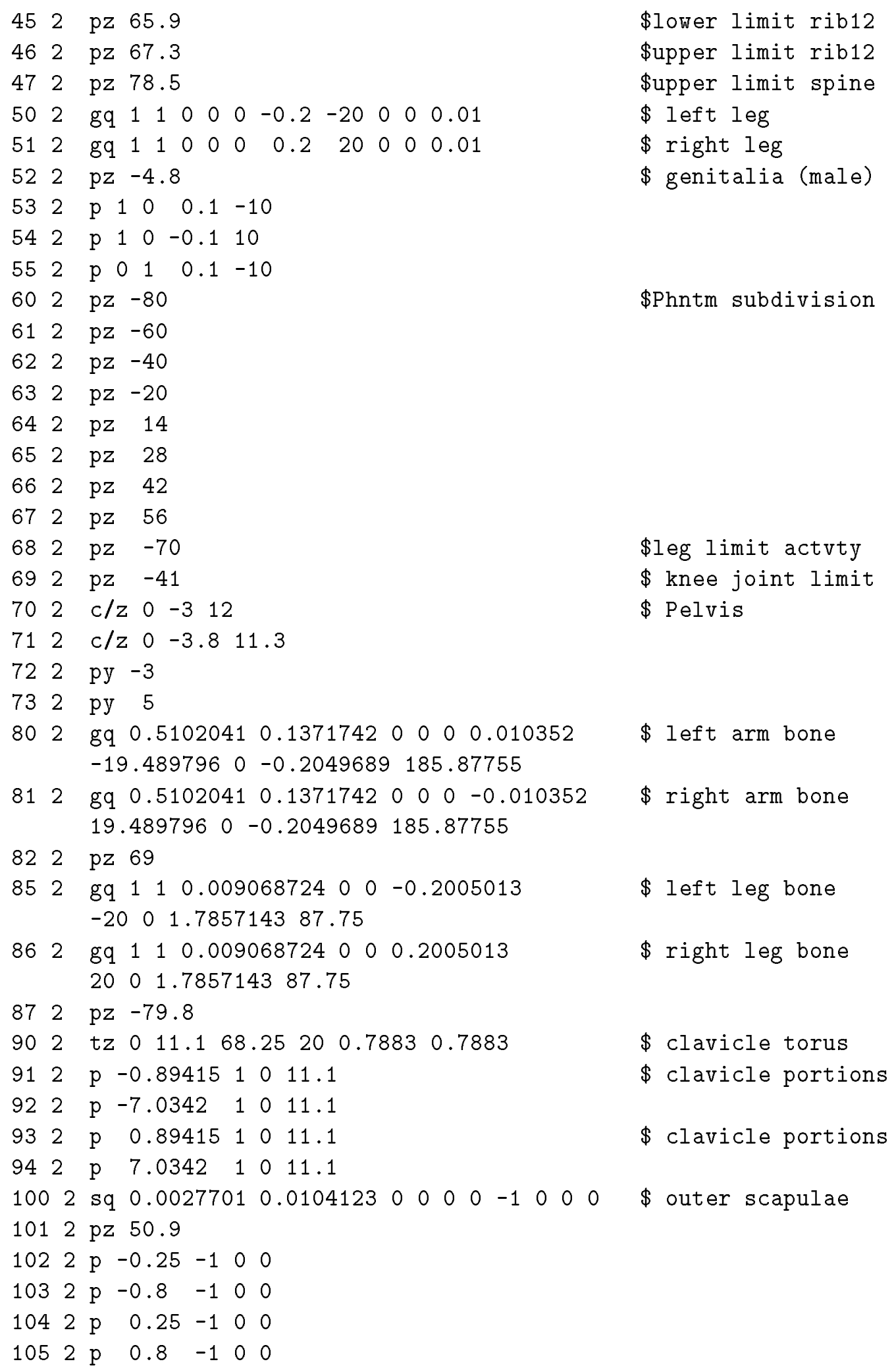




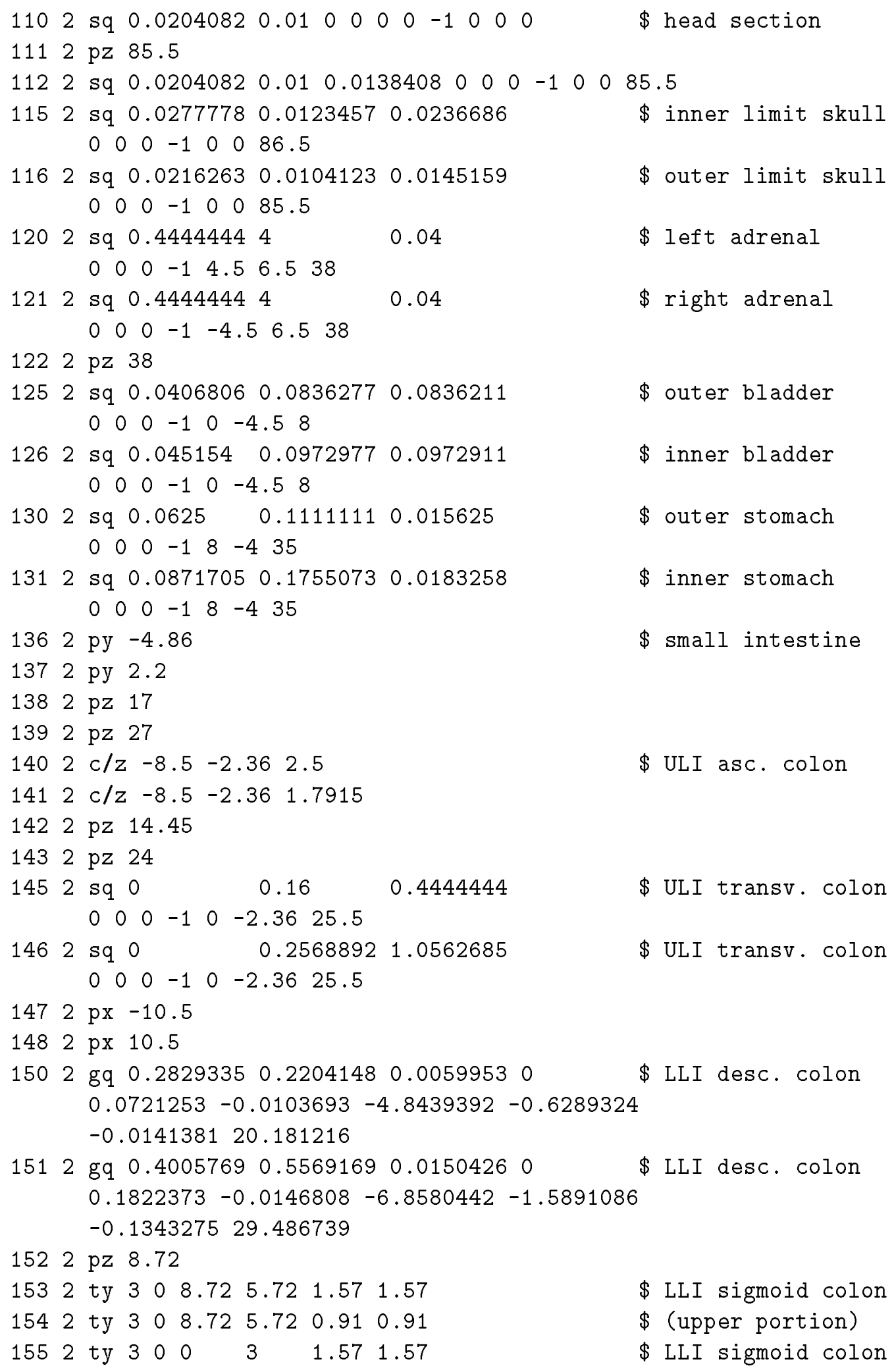




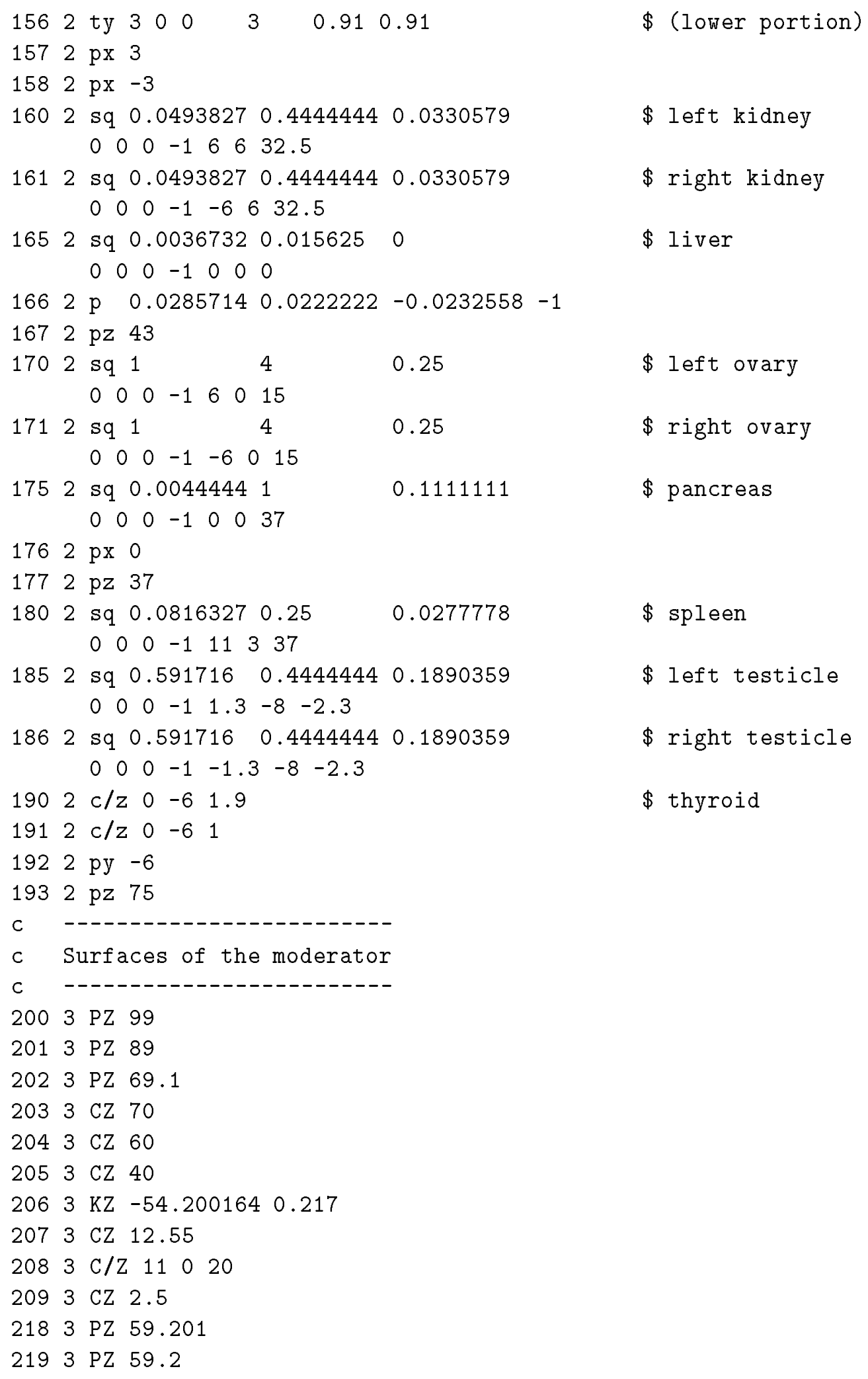




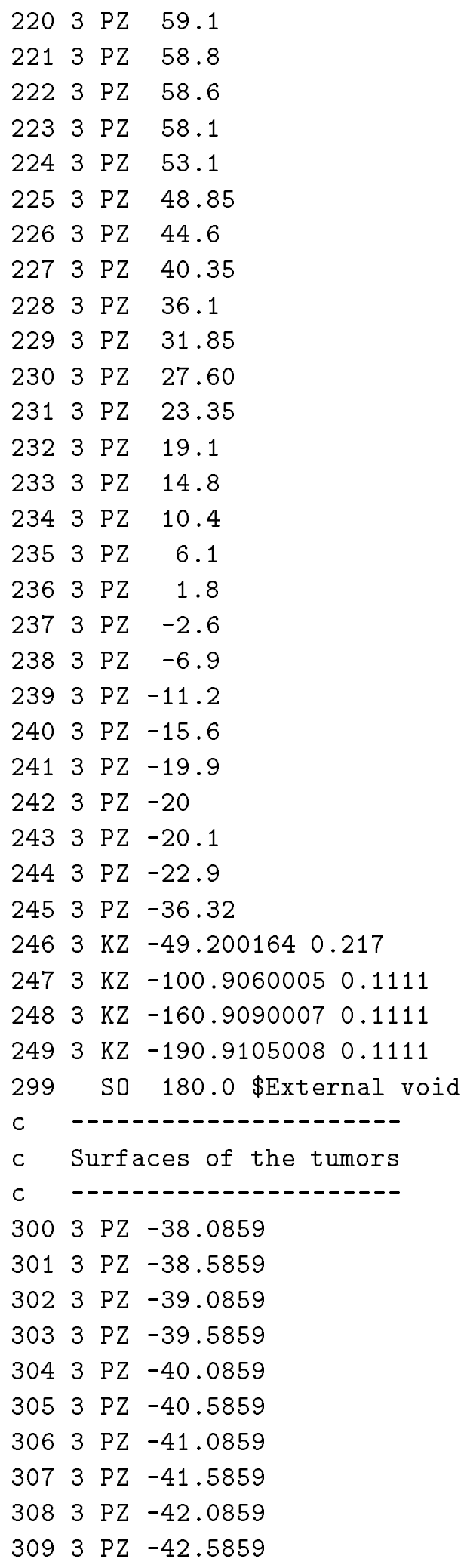




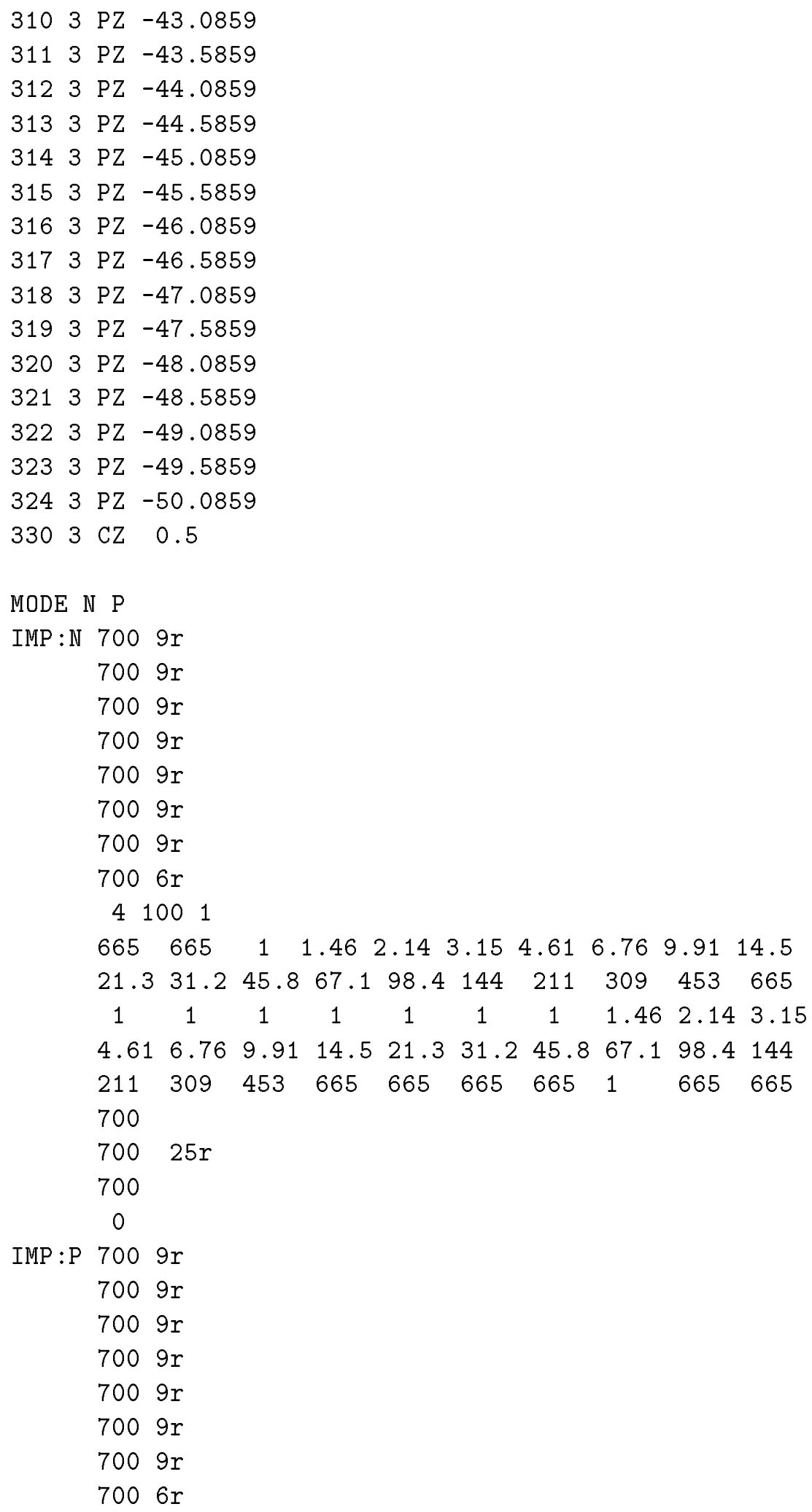




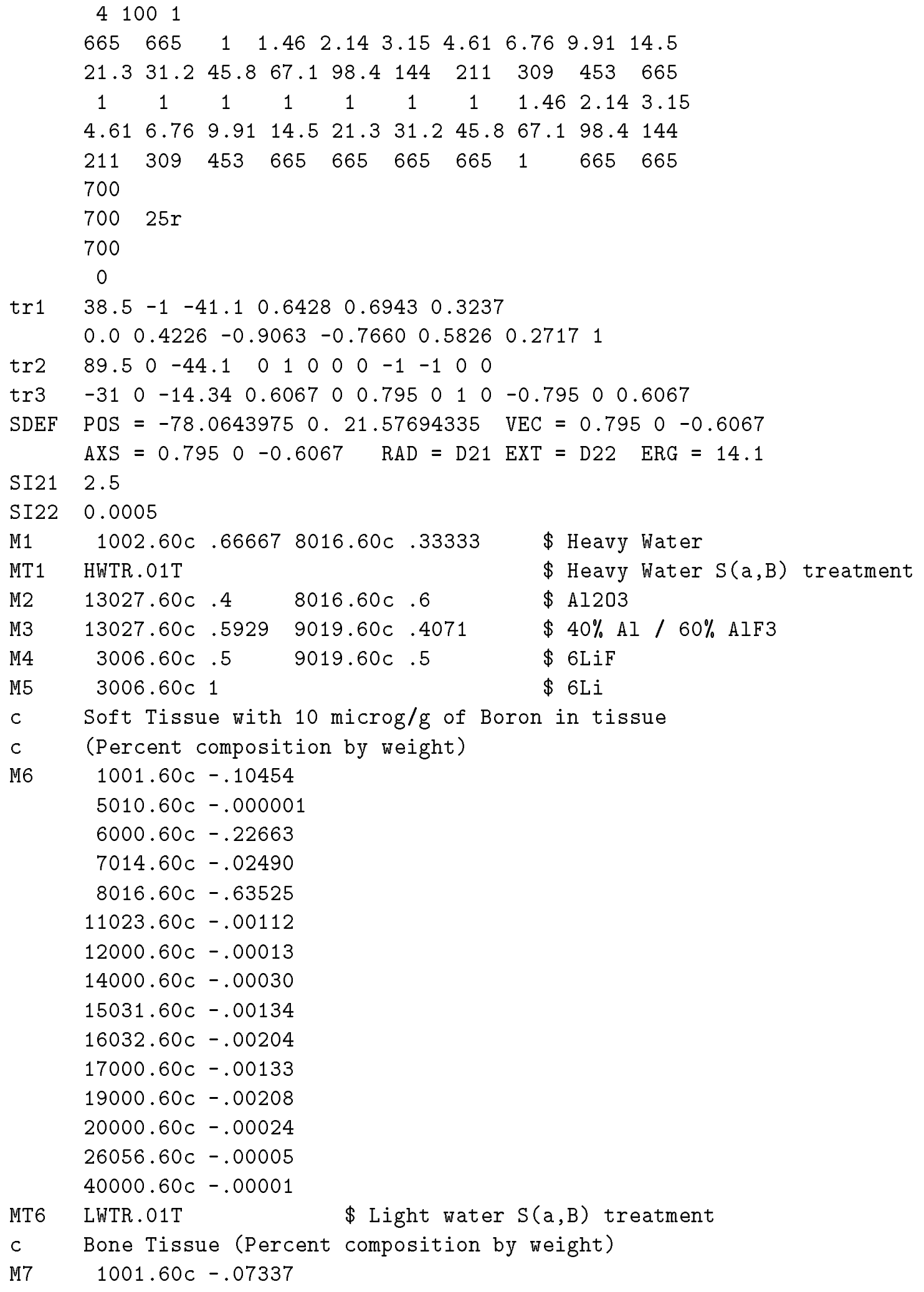




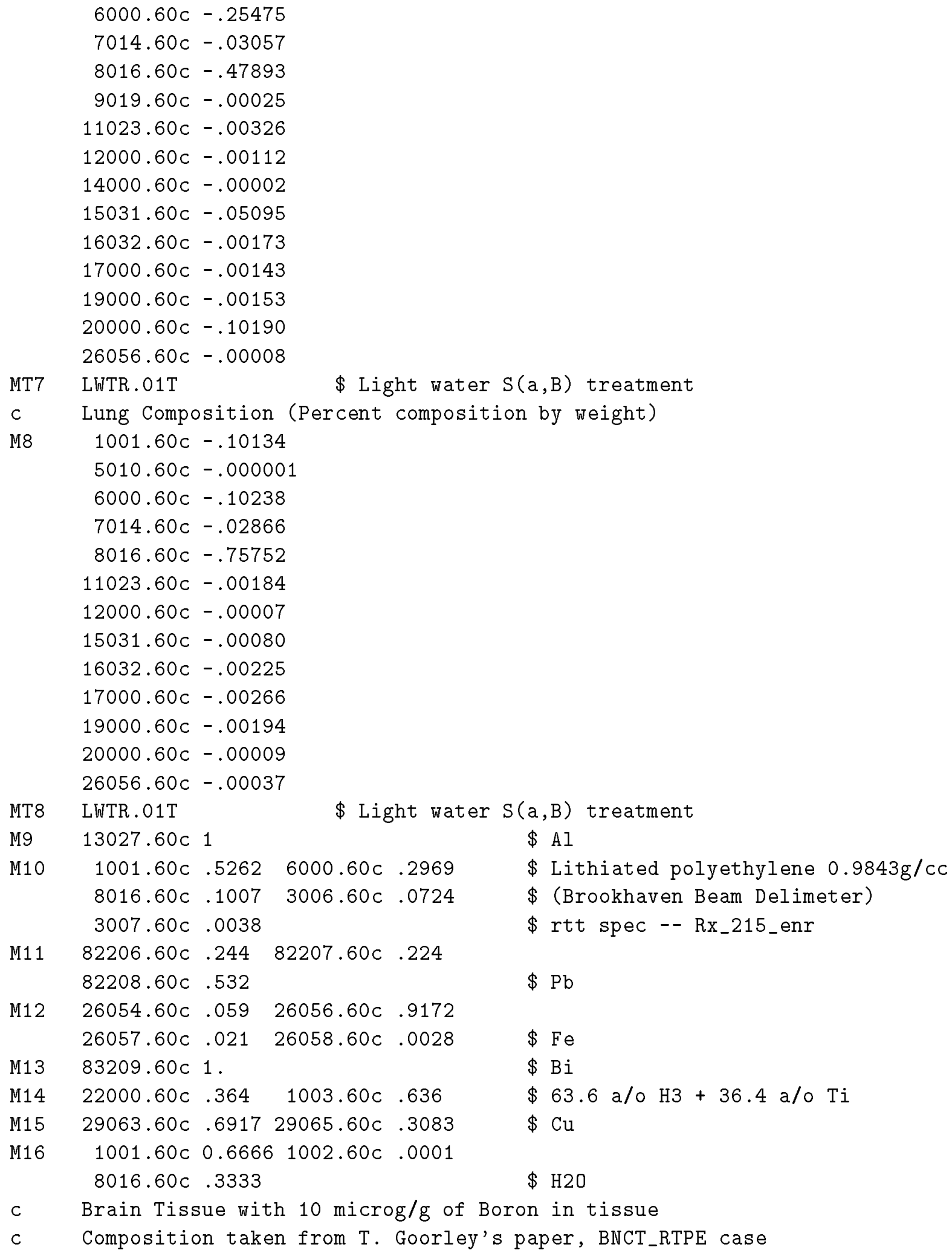




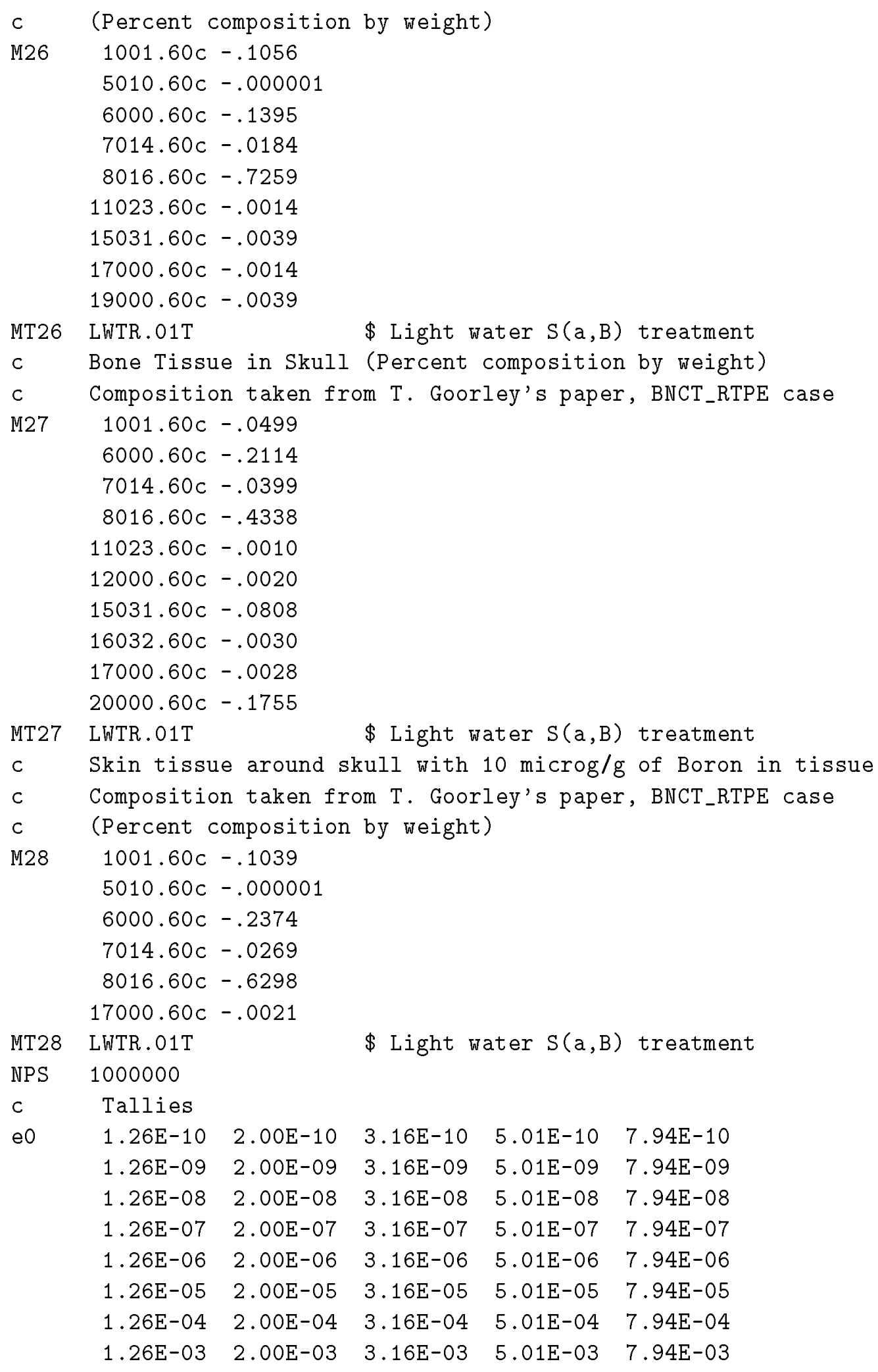




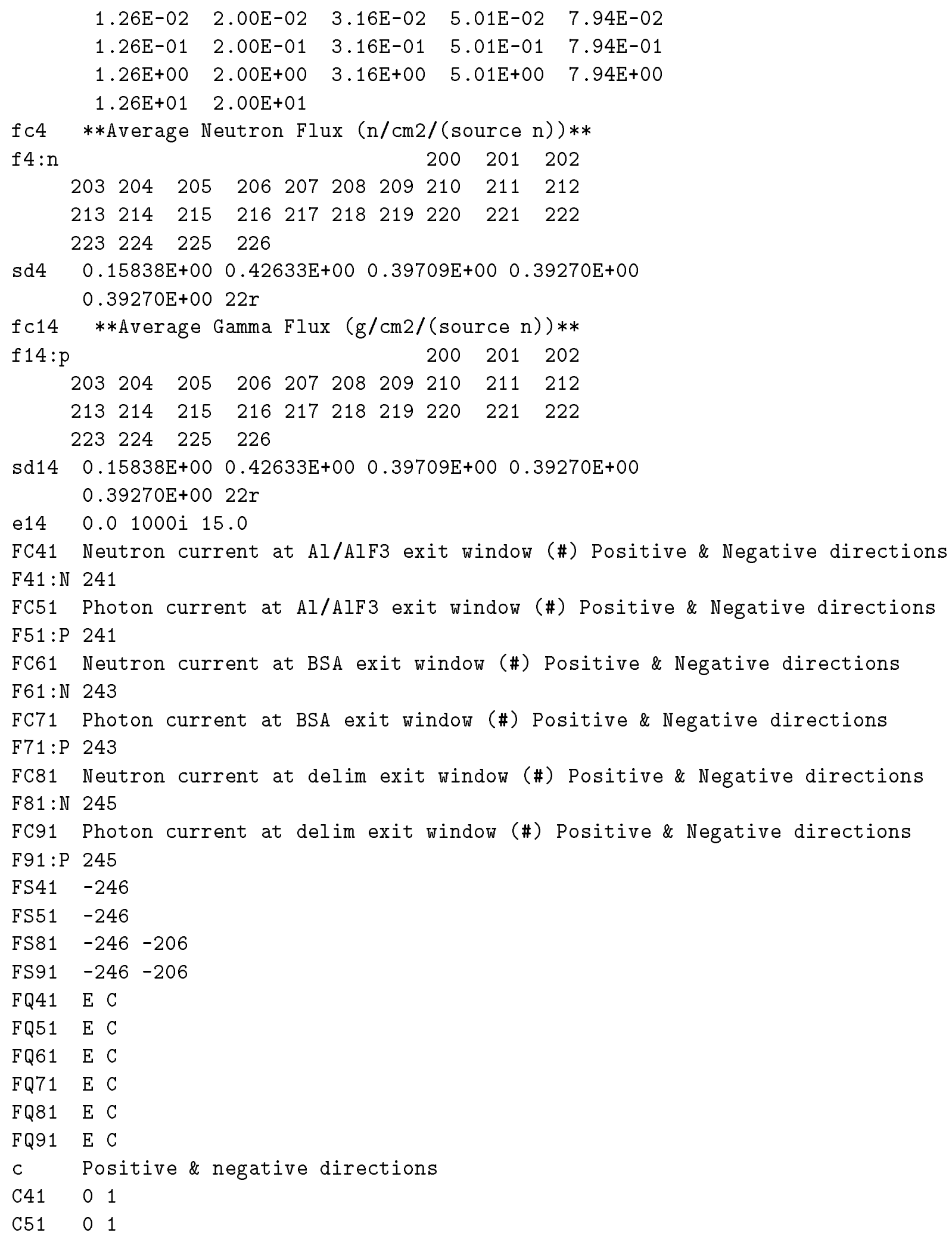




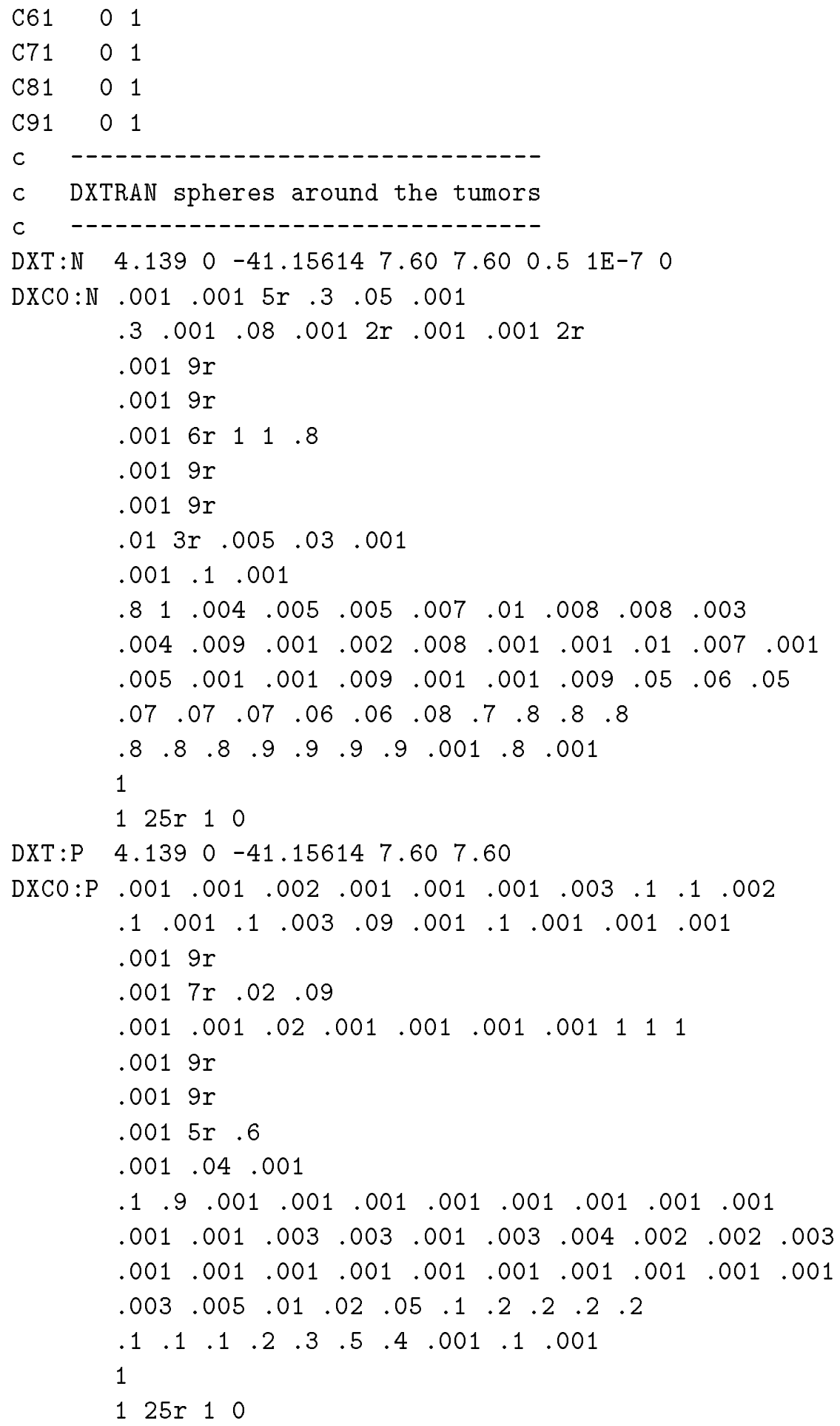




\section{C.2 MCNP input file corresponding to the MIRD 5 phantom}

\section{head shown in Fig. 3.1b and the BSA used in Sec. 4.8.3.}

- Dose calculation in Mird4 phantom due to the neutron beam 5/19/98 -

c The design was rotated of an angle of 52.65 degree in order to have an

$c$ irradiation of the brain tumors with a minimum shower effect.

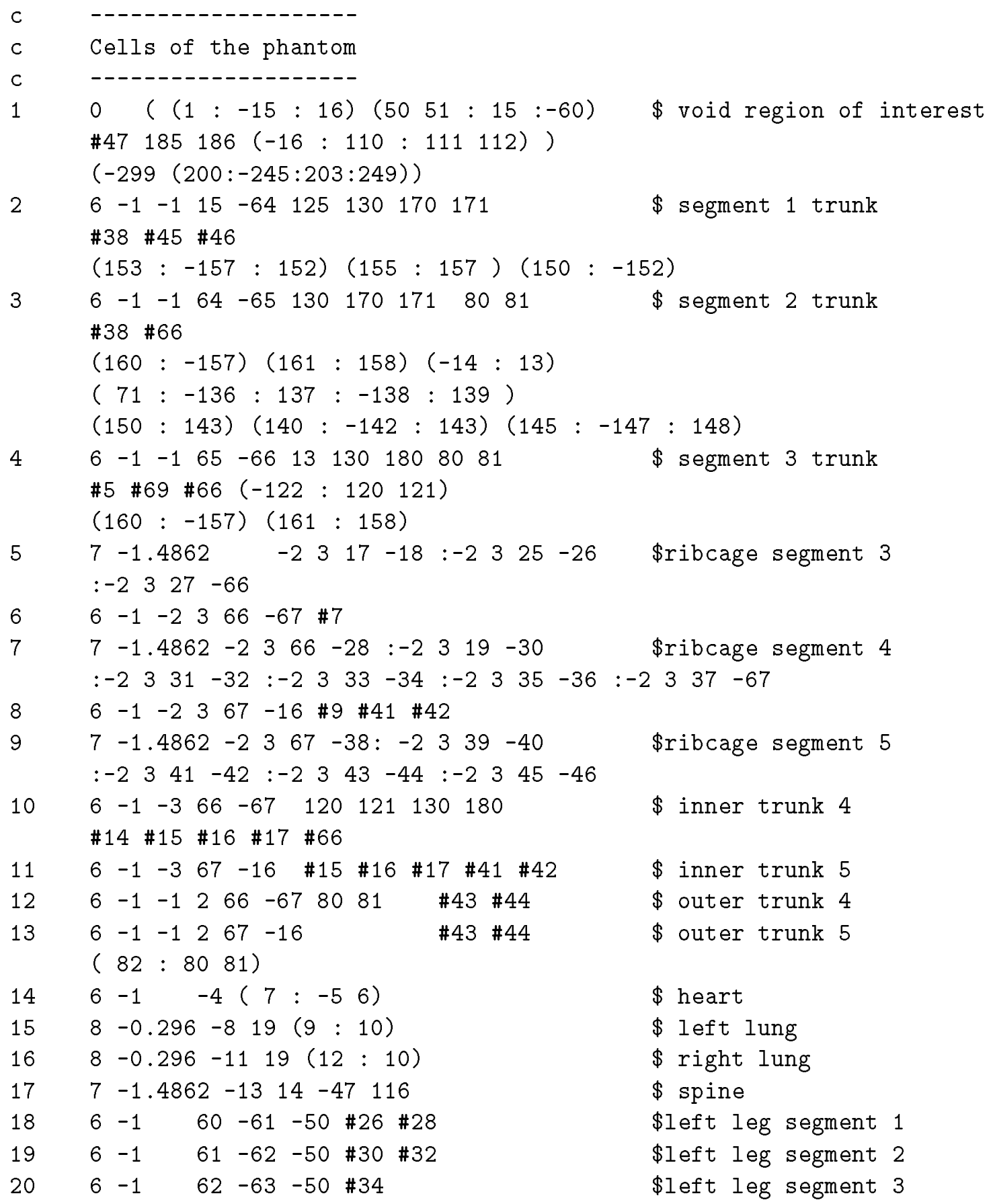




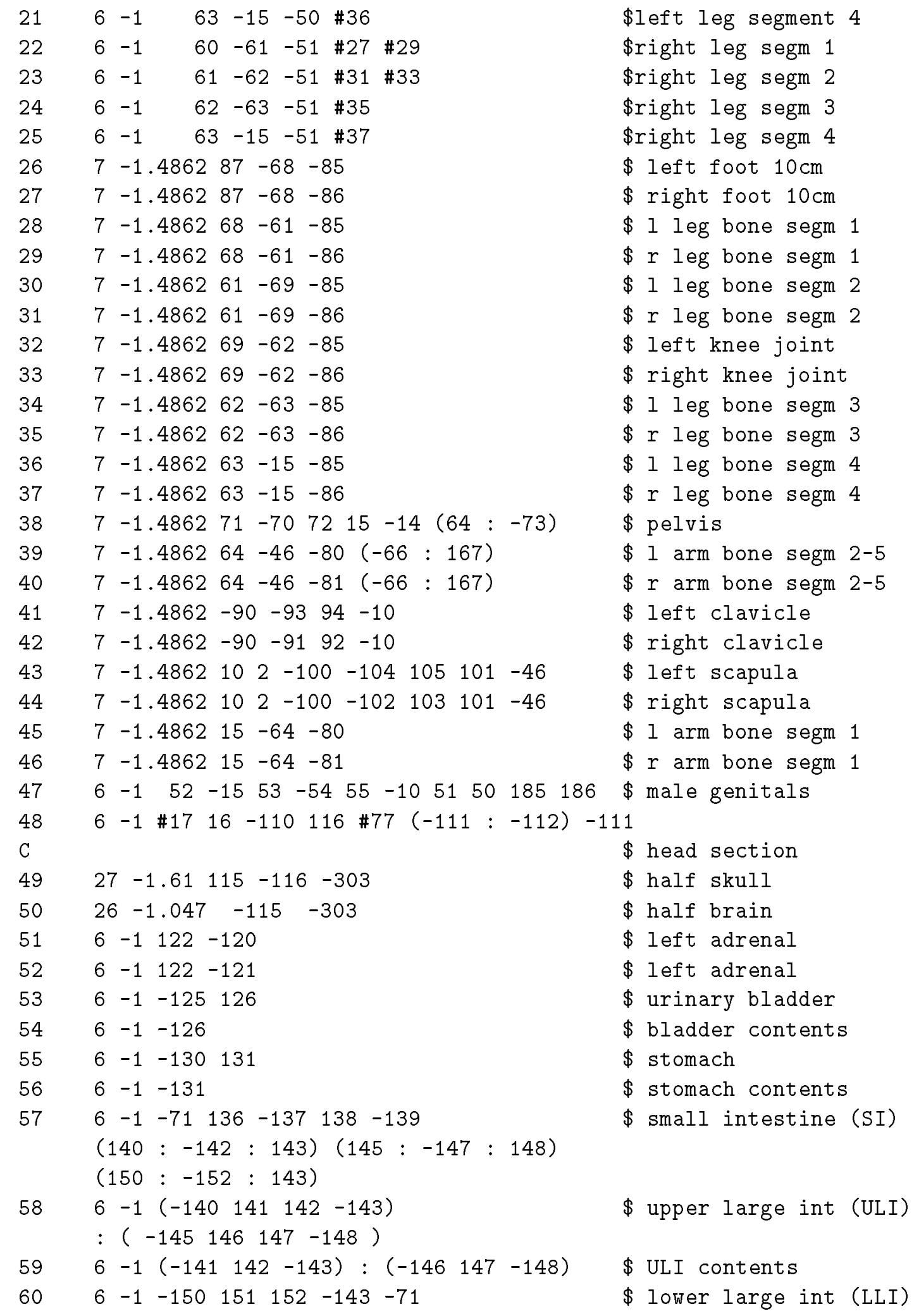




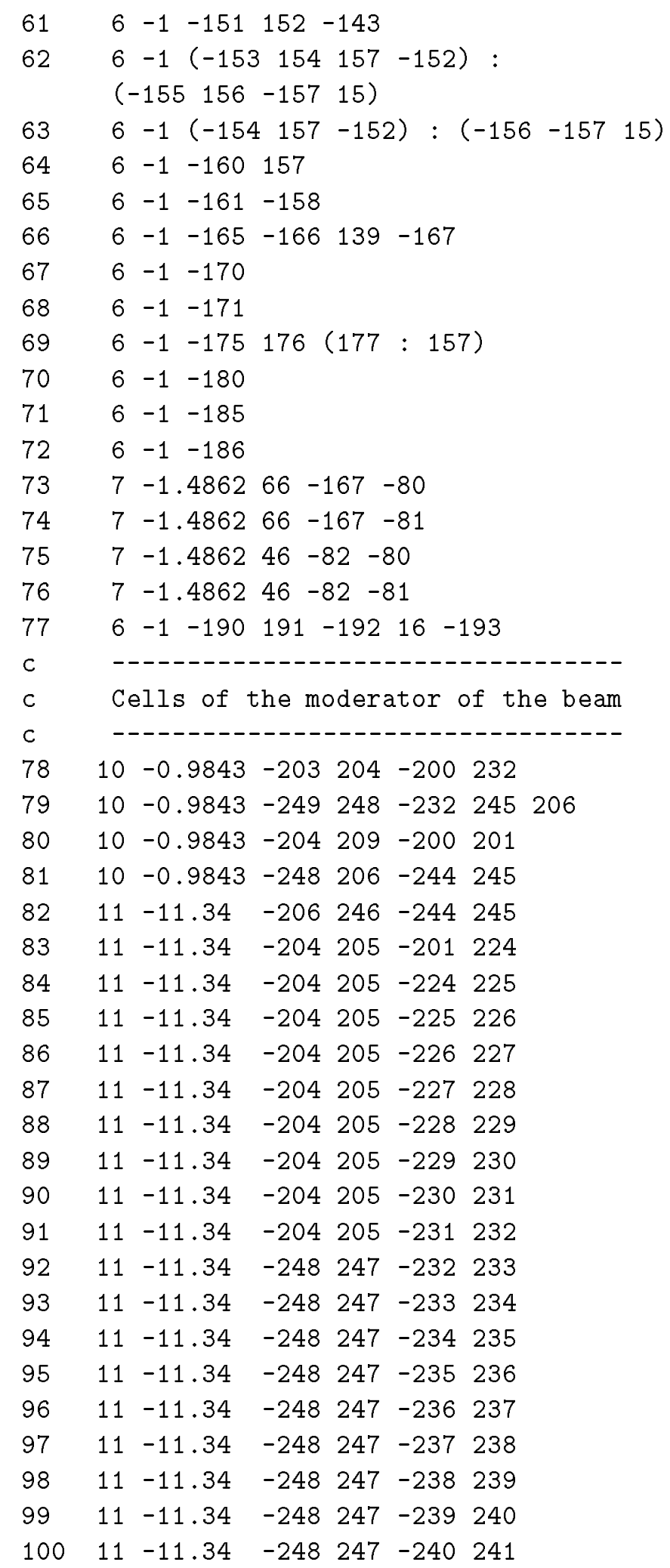

\author{
\$ LLI contents \\ \$ LLI sigmoid colon \\ \$ sigmoid colon contents \\ $\$$ left kidney \\ $\$$ right kidney \\ $\$$ liver \\ $\$$ left ovary \\ $\$$ right ovary \\ $\$$ pancreas \\ $\$$ spleen \\ $\$$ left testicle \\ $\$$ right testicle \\ $\$ 1$ arm bone elbow \\ \$ r arm bone elbow \\ $\$ 1$ arm bone shoulder \\ $\$ r$ arm bone shoulder \\ $\$$ thyroid
}




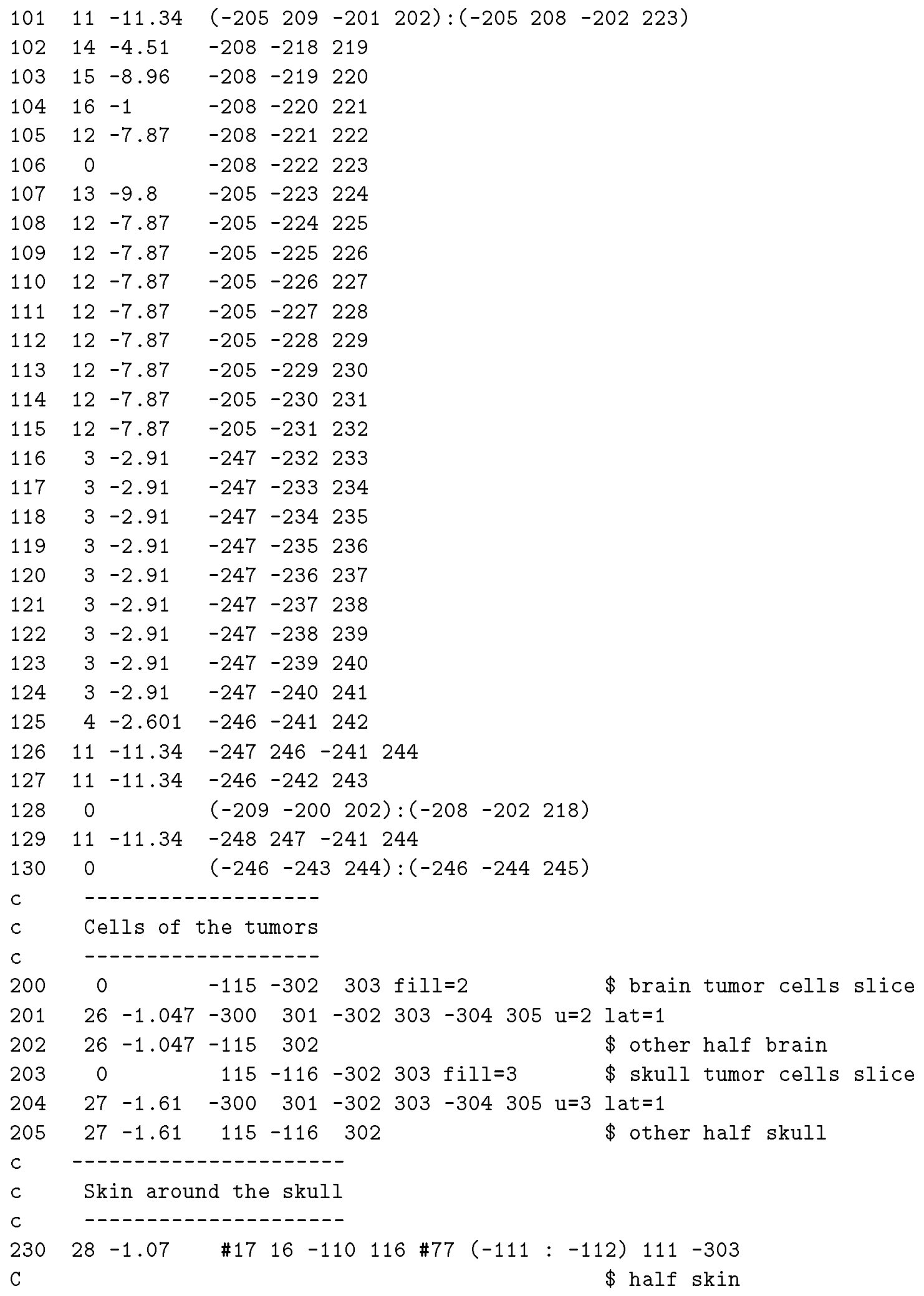




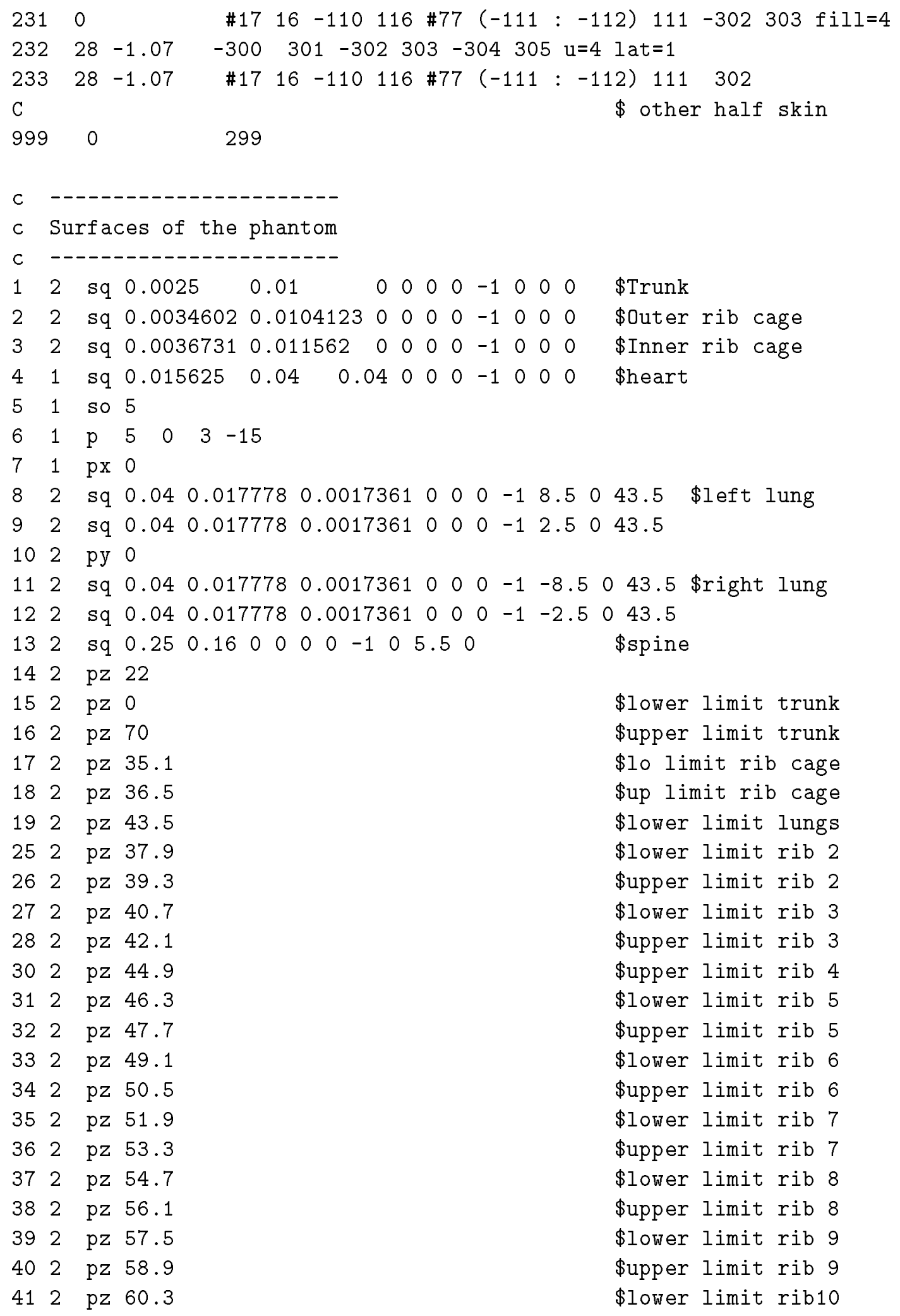




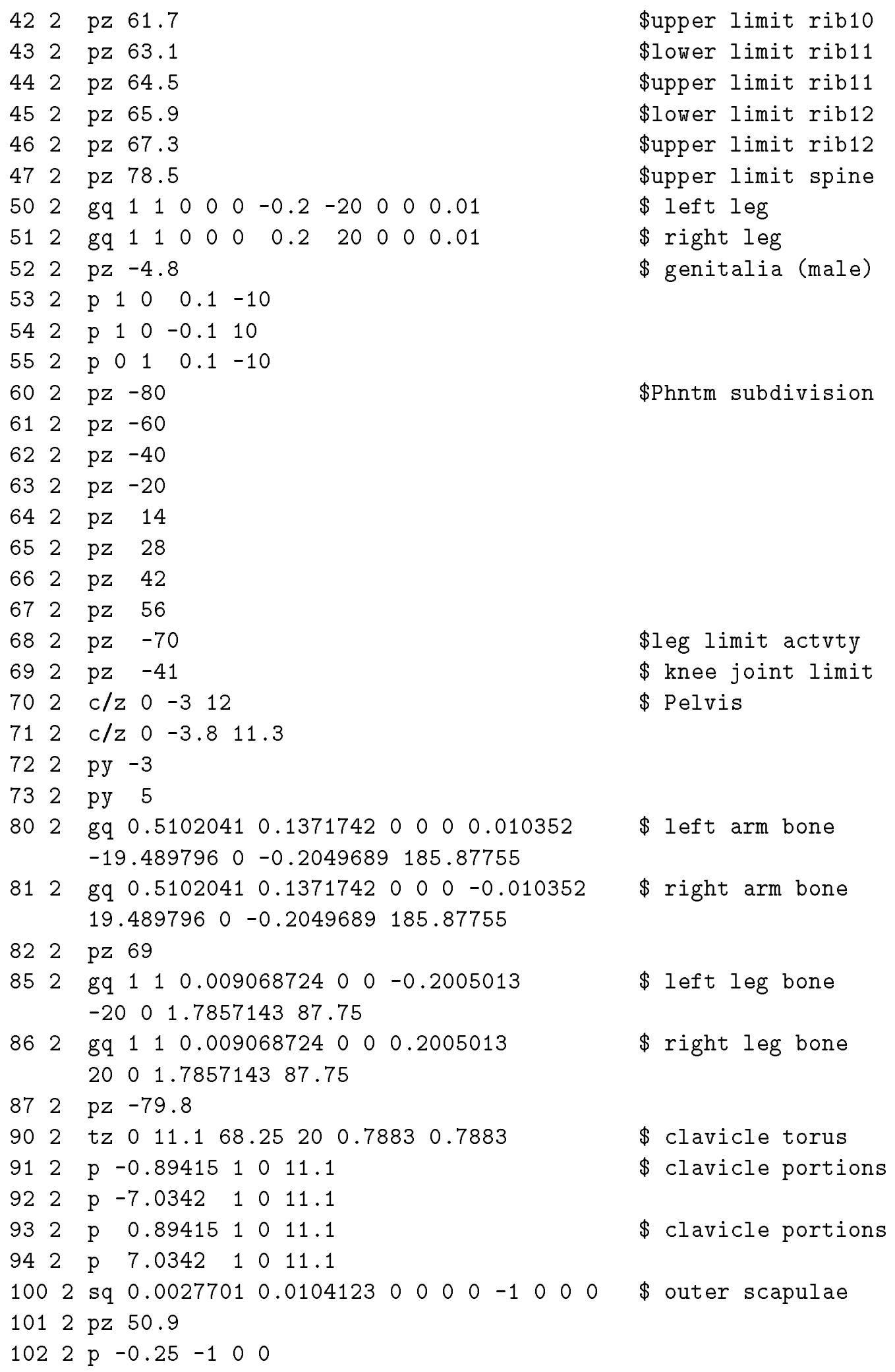




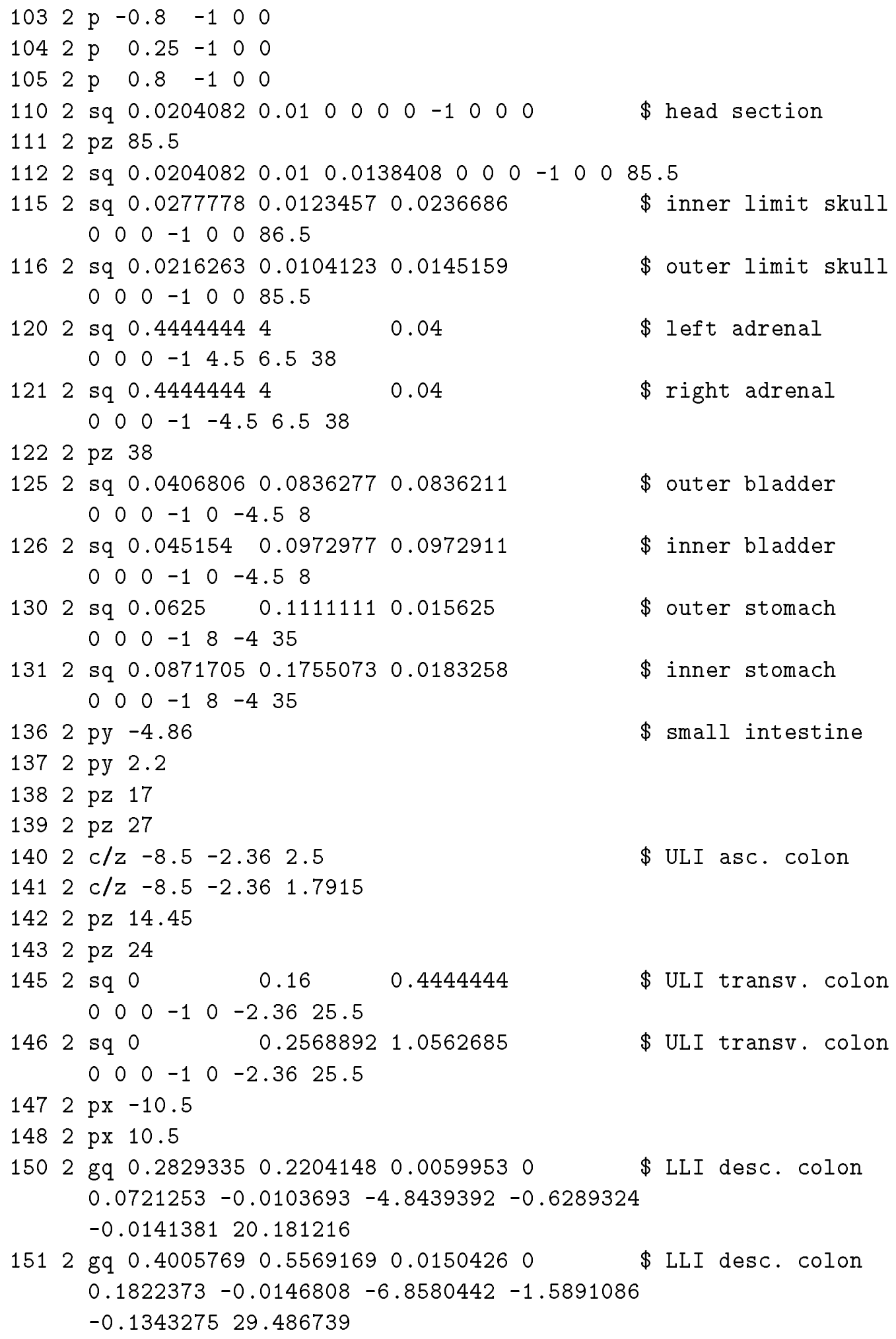




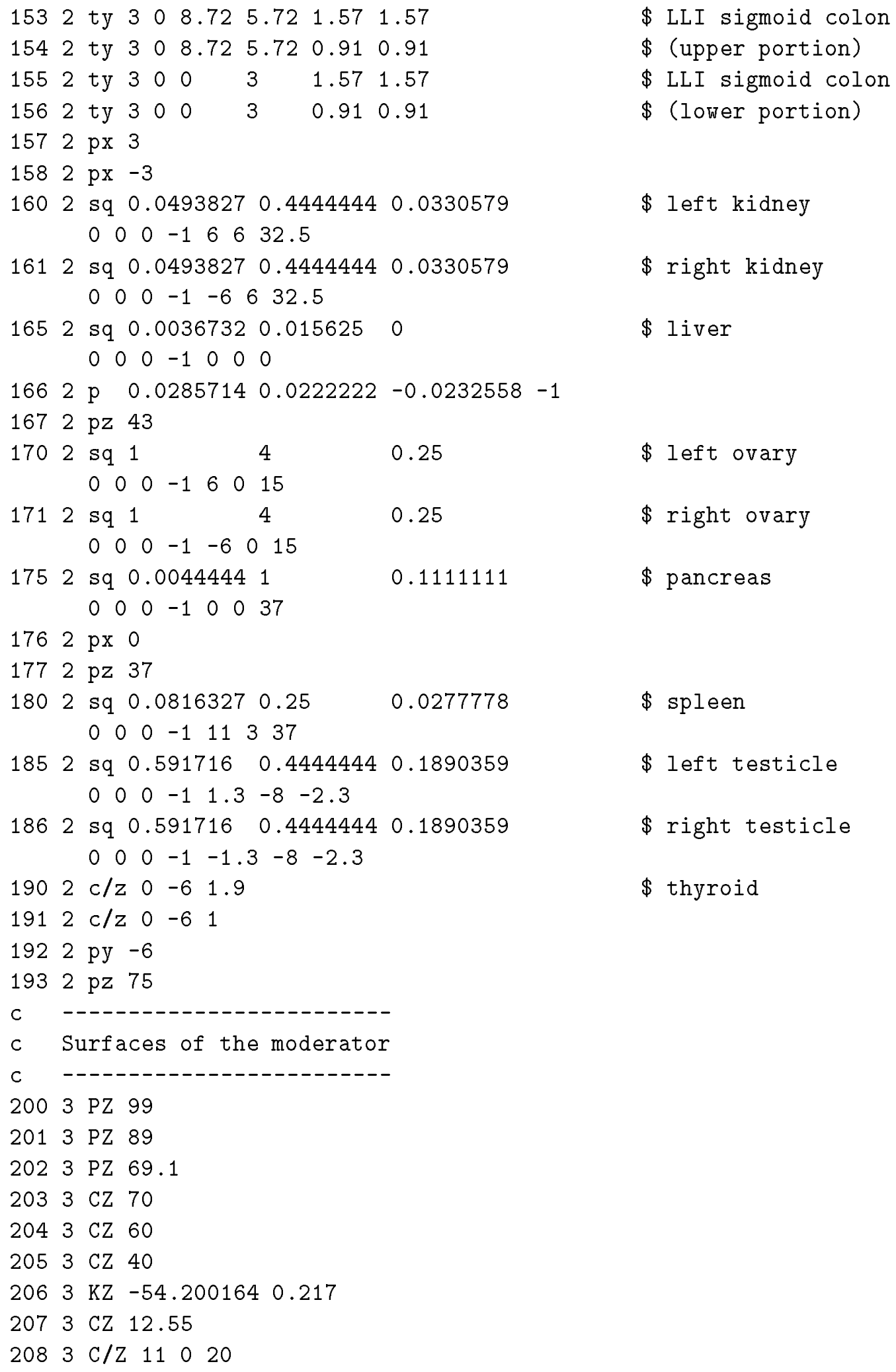

$0.1111111 \quad \$$ pancreas

\$ LLI sigmoid colon

\$ (upper portion)

\$ LLI sigmoid colon

\$ (lower portion)

\$ liver

$\$$ thyroid 


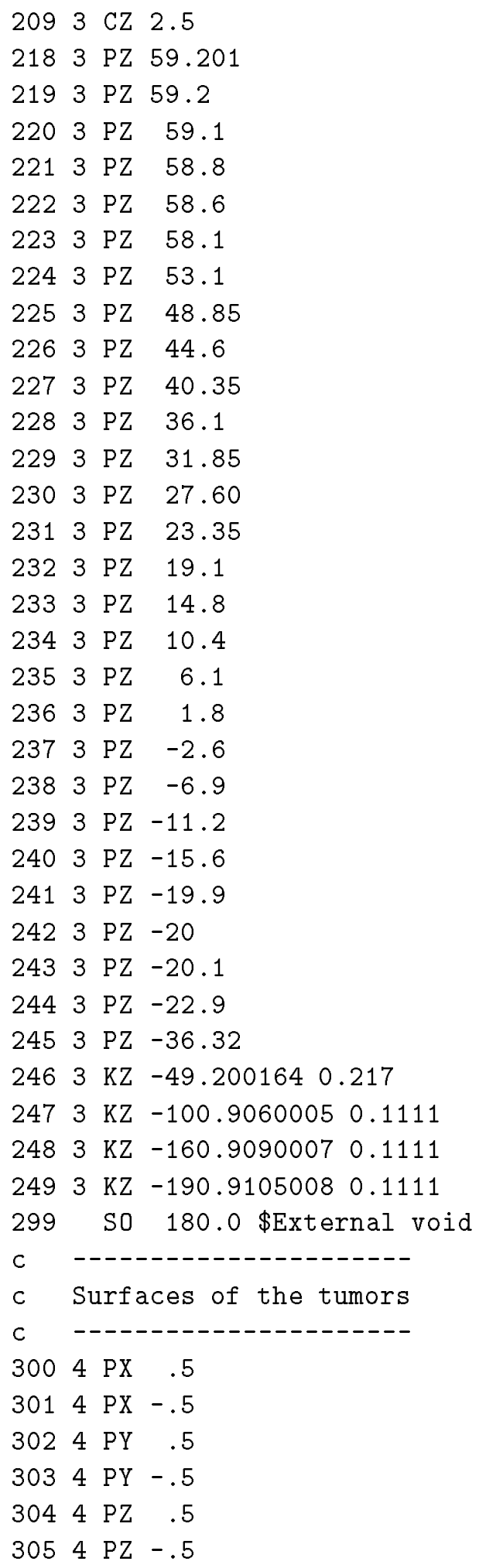




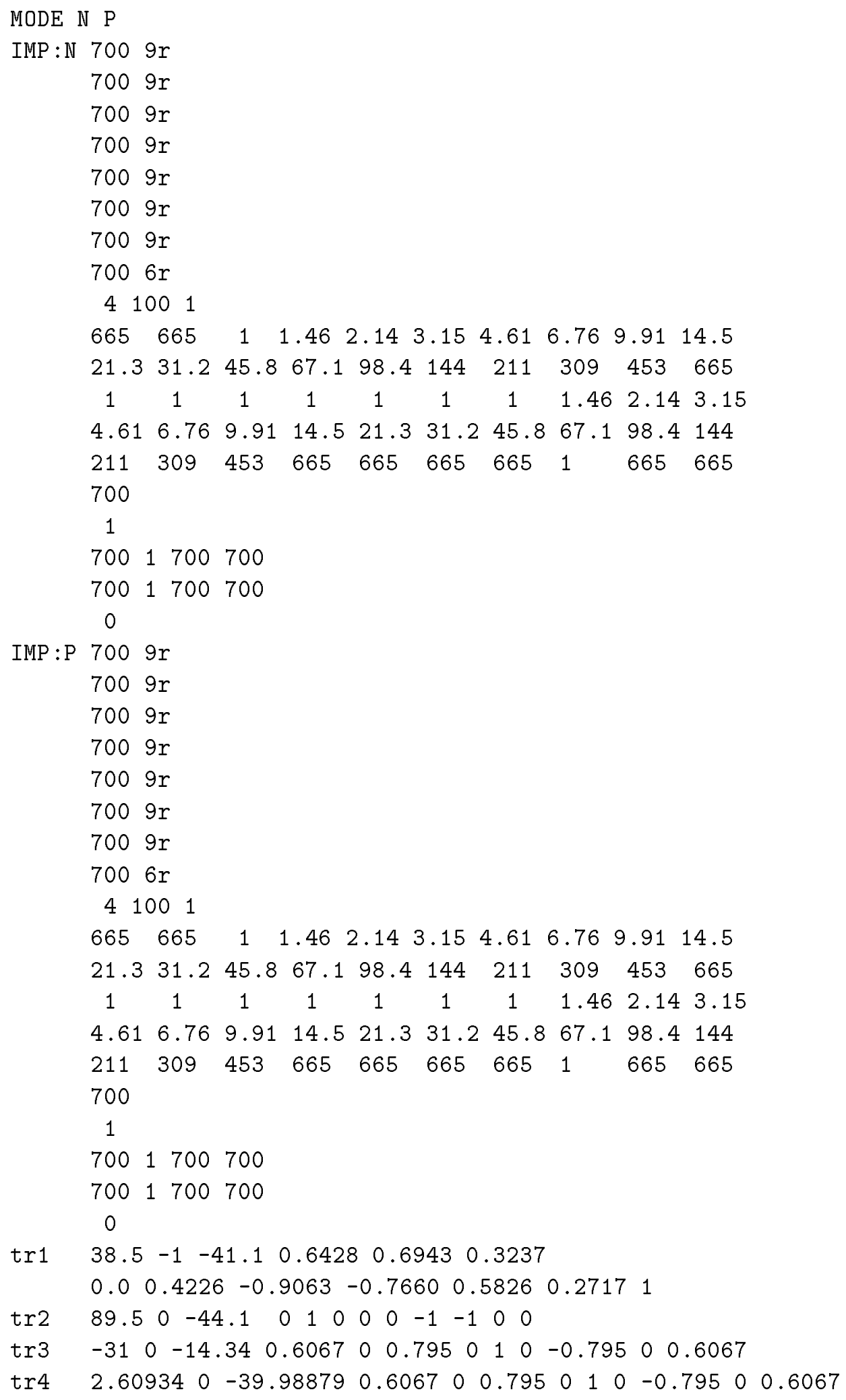




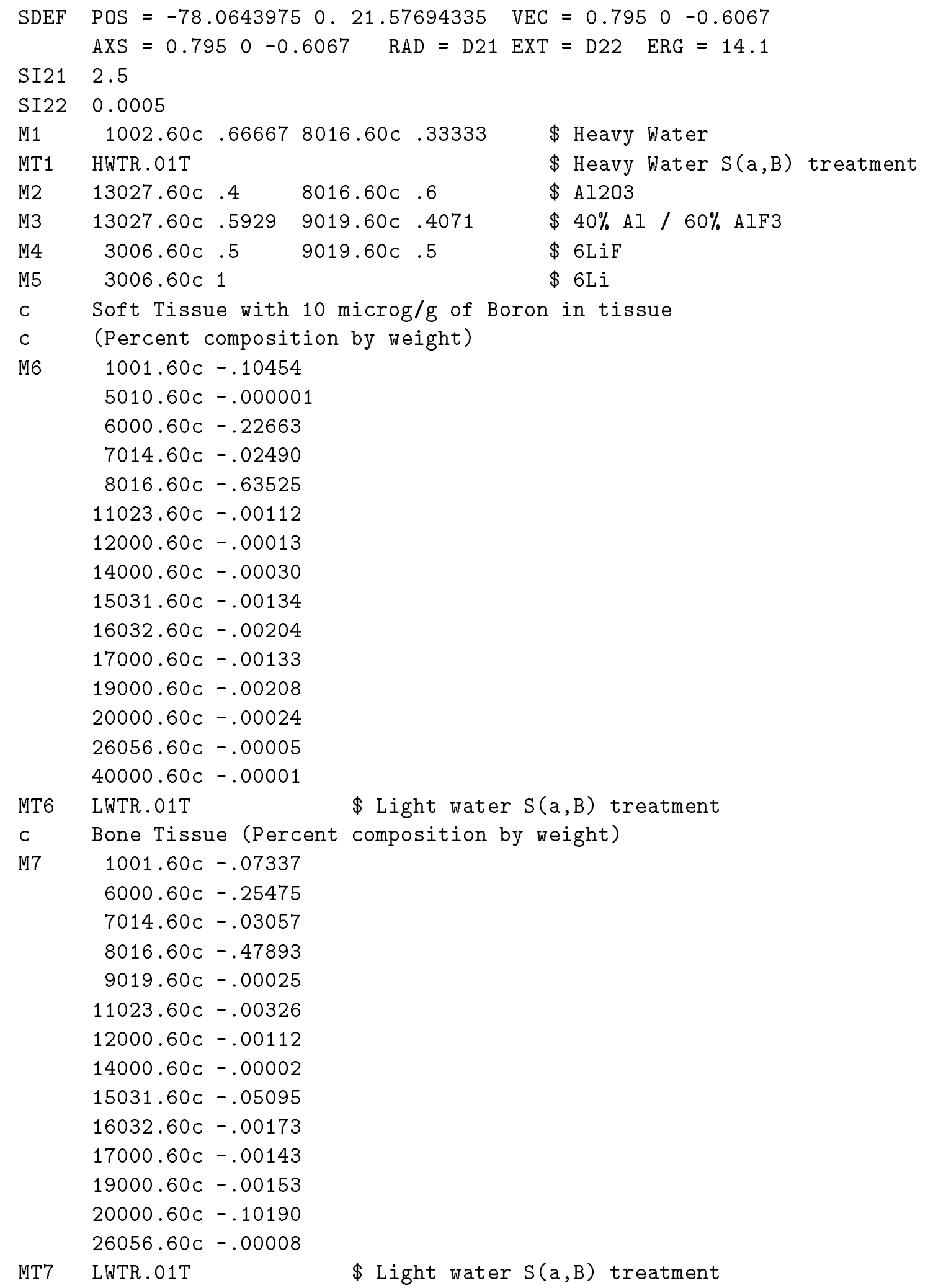




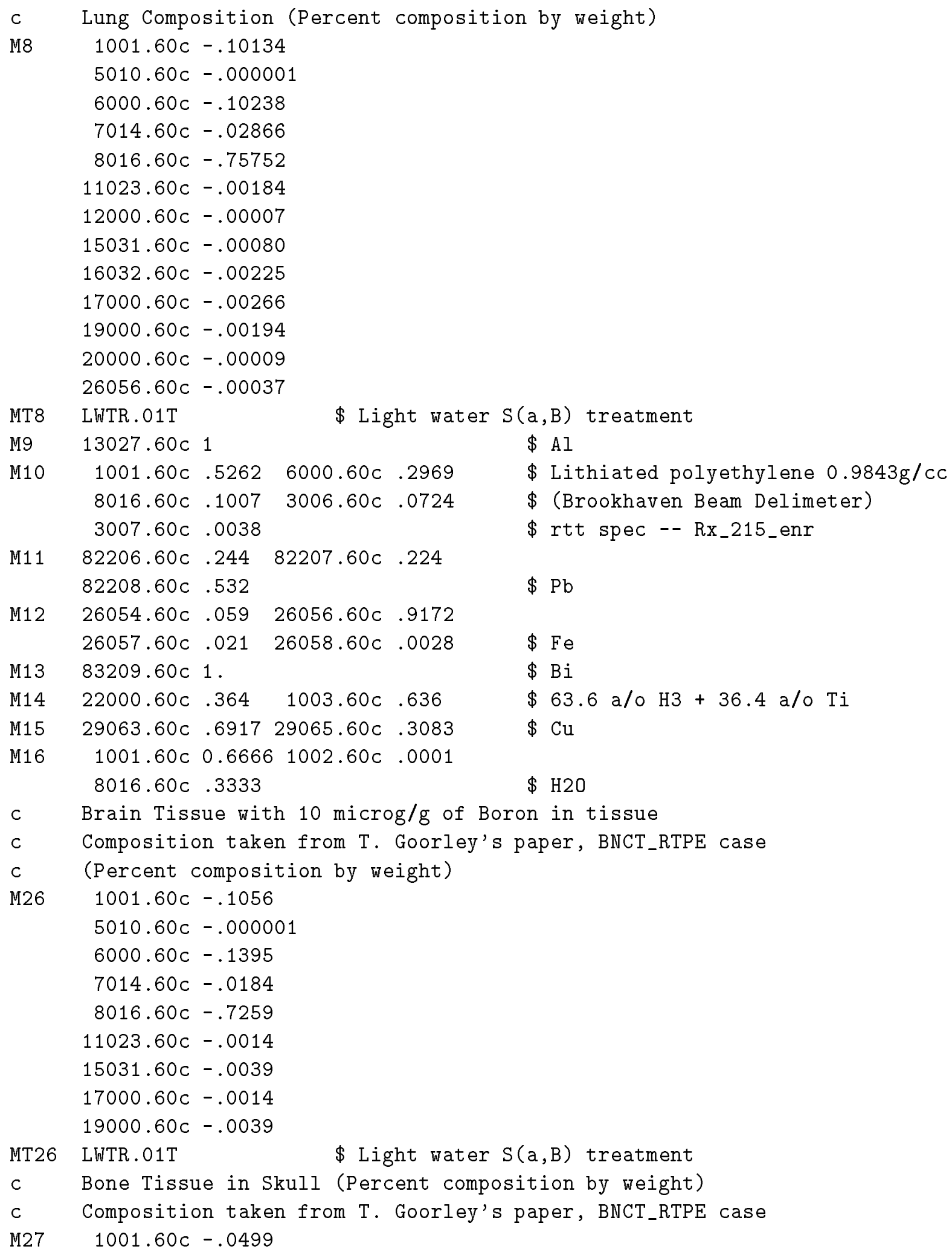




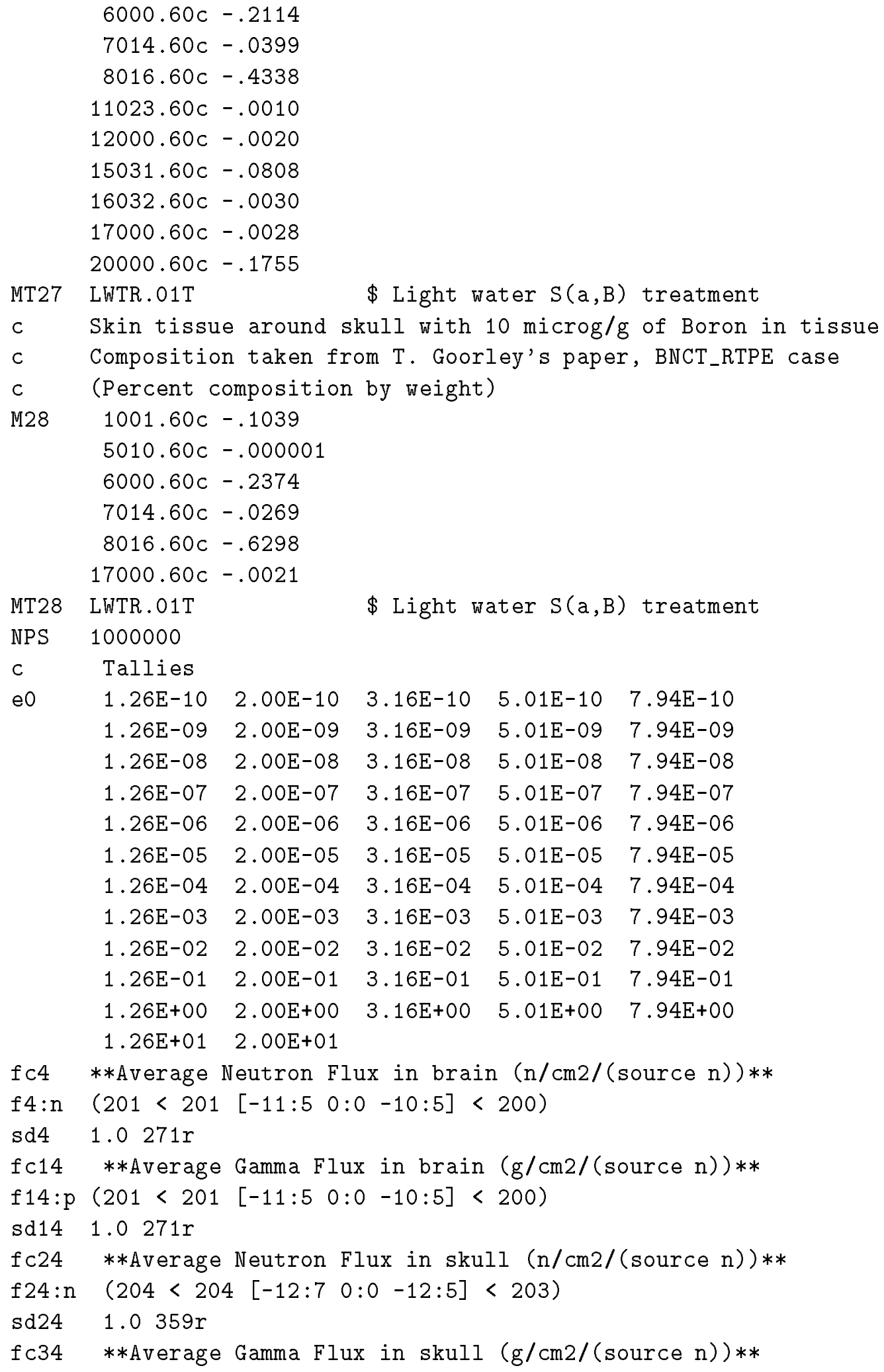




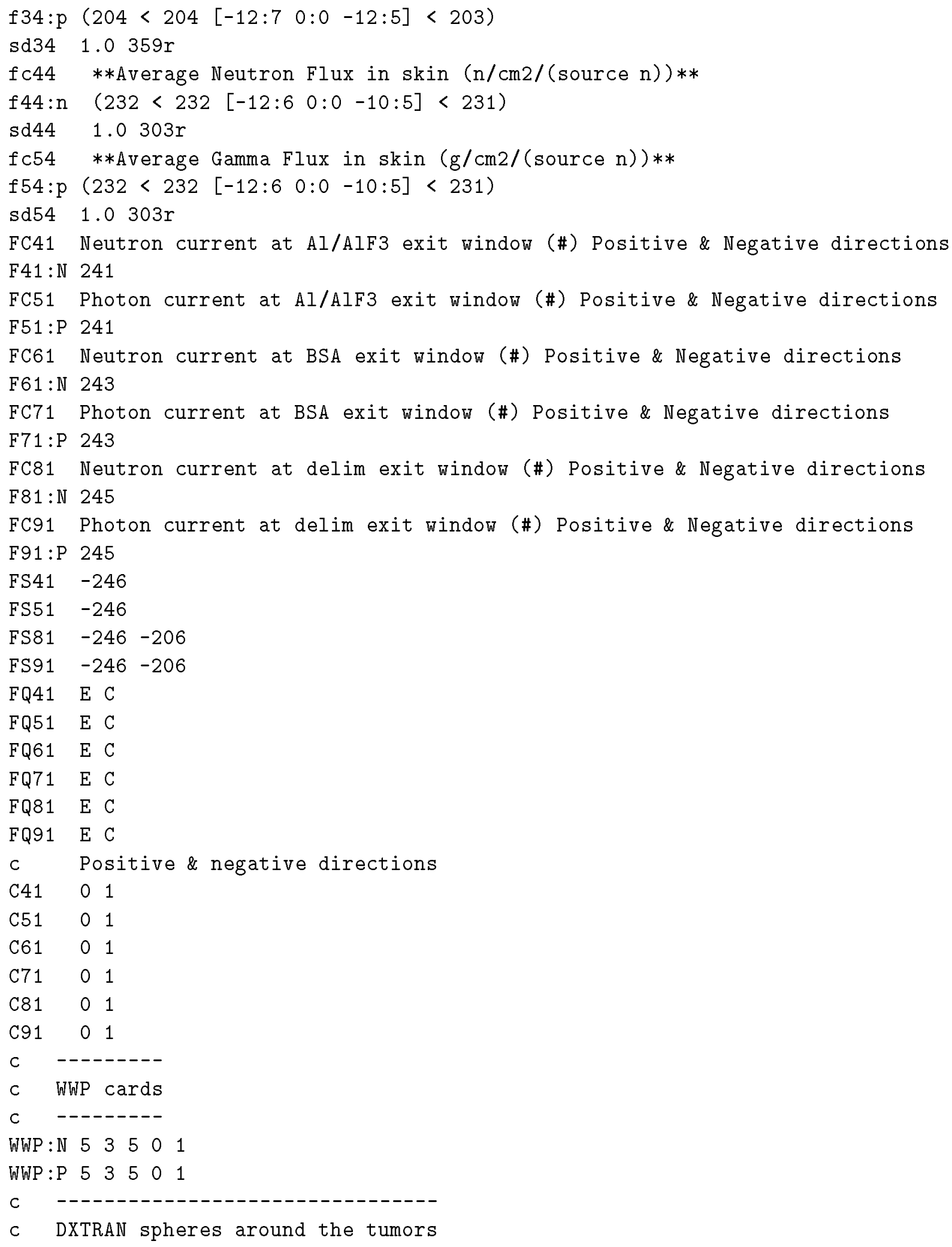




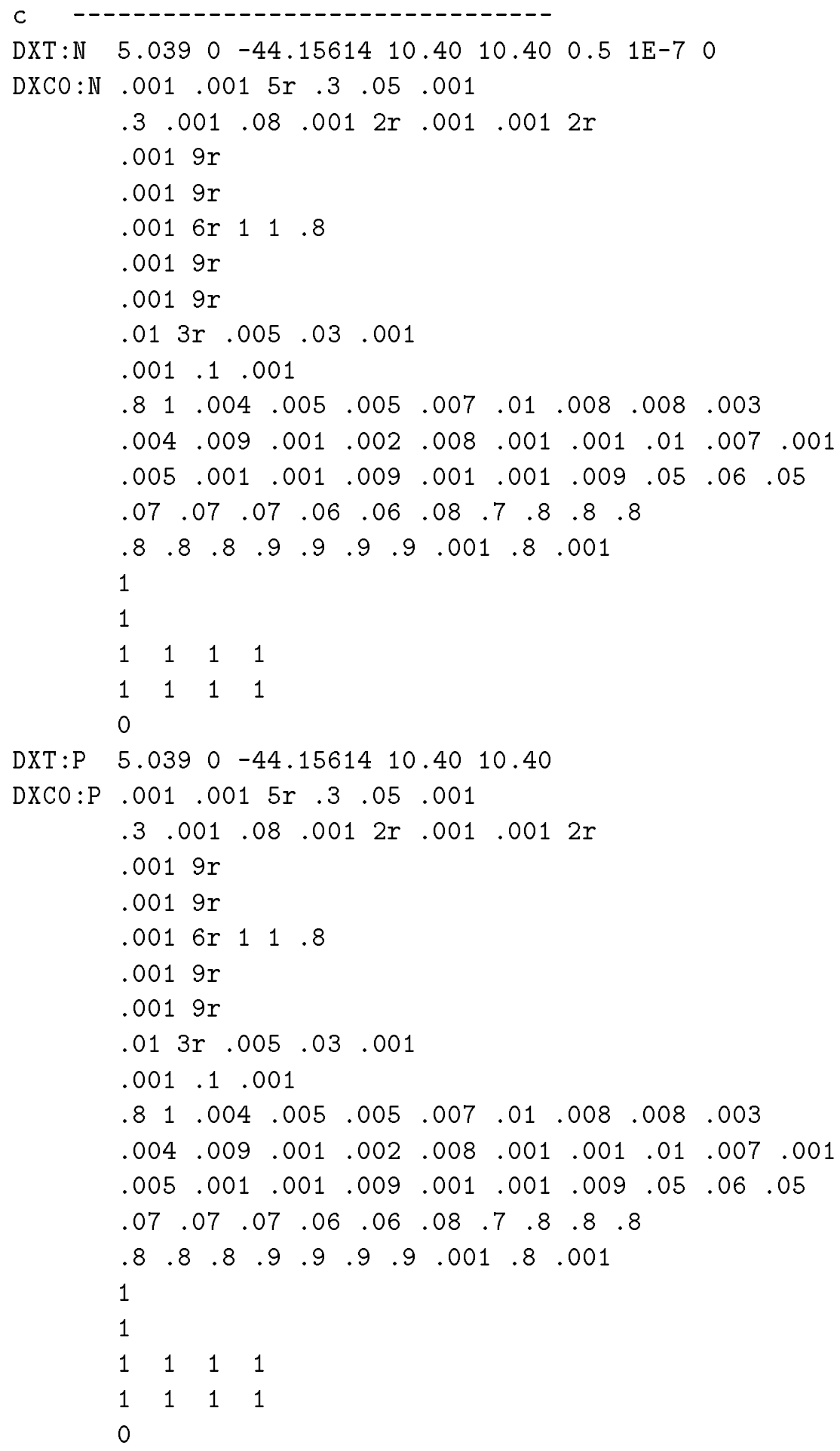

